NBER WORKING PAPER SERIES

\title{
FISCAL UNIONS
}

Ivan Werning

Emmanuel Farhi

Working Paper 18280

http://www.nber.org/papers/w18280

\section{NATIONAL BUREAU OF ECONOMIC RESEARCH \\ 1050 Massachusetts Avenue \\ Cambridge, MA 02138}

August 2012, Revised March 2017

For useful comments and conversations we thank Fernando Alvarez, George-Marios Angeletos, Marco Bassetto, Giancarlo Corsetti, Jordi Gali, Pierre-Oliver Gourinchas, Olivier Jeanne, Patrick Kehoe, Guido Lorenzoni, Tomaso Monacelli, Maurice Obstfeld, Kenneth Rogoff, Robert Staiger and Jean Tirole. We thank seminar participants at Bocconi, Brown, Chicago, Columbia, CREI, Harvard, LSE, MIT, Princeton, University of Wisconsin, Wharton, Bank of England, ECB, IMF, NBER, NY Fed, SITE. We thank Andreas Schaab for superlative research assistance. The views expressed herein are those of the authors and do not necessarily reflect the views of the National Bureau of Economic Research.

NBER working papers are circulated for discussion and comment purposes. They have not been peer-reviewed or been subject to the review by the NBER Board of Directors that accompanies official NBER publications.

(C) 2012 by Ivan Werning and Emmanuel Farhi. All rights reserved. Short sections of text, not to exceed two paragraphs, may be quoted without explicit permission provided that full credit, including (C) notice, is given to the source. 
Fiscal Unions

Ivan Werning and Emmanuel Farhi

NBER Working Paper No. 18280

August 2012, Revised March 2017

JEL No. E62,E63,F02,F15,F3,F40

\begin{abstract}
$\underline{\text { ABSTRACT }}$
We study cross-country risk sharing as a second-best problem for members of a currency union using an open economy model with nominal rigidities and provide two key results. First, we show that if financial markets are incomplete, the value of gaining access to any given level of aggregate risk sharing is greater for countries that are members of a currency union. Second, we show that even if financial markets are complete, privately optimal risk sharing is constrained inefficient. A role emerges for government intervention in risk sharing both to guarantee its existence and to influence its operation. The constrained efficient risk sharing arrangement can be implemented by contingent transfers within a fiscal union. We find that the benefits of such a fiscal union are larger, the more asymmetric the shocks affecting the members of the currency union, the more persistent these shocks, and the less open the member economies. Finally we compare the performance of fiscal unions and of other macroeconomic stabilization instruments available in currency unions such as capital controls, government spending, fiscal deficits, and redistribution.
\end{abstract}

Ivan Werning

Department of Economics, E52-536

MIT

77 Massachusetts Avenue

Cambridge, MA 02139

and NBER

iwerning@mit.edu

Emmanuel Farhi

Harvard University

Department of Economics

Littauer Center

Cambridge, MA 02138

and NBER

emmanuel.farhi@gmail.com 


\title{
Fiscal Unions*
}

\author{
Emmanuel Farhi \\ Harvard University
}

\section{Iván Werning MIT}

\section{March 2017}

\begin{abstract}
We study cross-country risk sharing as a second-best problem for members of a currency union using an open economy model with nominal rigidities and provide two key results. First, we show that if financial markets are incomplete, the value of gaining access to any given level of aggregate risk sharing is greater for countries that are members of a currency union. Second, we show that even if financial markets are complete, privately optimal risk sharing is constrained inefficient. A role emerges for government intervention in risk sharing both to guarantee its existence and to influence its operation. The constrained efficient risk sharing arrangement can be implemented by contingent transfers within a fiscal union. We find that the benefits of such a fiscal union are larger, the more asymmetric the shocks affecting the members of the currency union, the more persistent these shocks, and the less open the member economies. Finally we compare the performance of fiscal unions and of other macroeconomic stabilization instruments available in currency unions such as capital controls, government spending, fiscal deficits, and redistribution.
\end{abstract}

\section{Introduction}

The benefits of flexible exchange rates were famously argued for by Friedman (1953) and are widely accepted by economists. Countries in a currency union forego the possibility of adjustments to their exchange rates in response to asymmetric shocks. How costly is this loss in flexibility and what can be done to compensate for it? These questions are precisely those tackled by the Optimal Currency Area (OCA) literature (for the pioneering articles, see Mundell, 1961; McKinnon, 1963; Kenen, 1969).

In a seminal contribution, Kenen (1969) argued that fiscal integration was critical to a wellfunctioning currency union:

"It is a chief function of fiscal policy, using both sides of the budget, to offset or compensate for regional differences, whether in earned income or in unemployment rates. The

*For useful comments and conversations we thank Fernando Alvarez, George-Marios Angeletos, Marco Bassetto, Giancarlo Corsetti, Jordi Gali, Pierre-Oliver Gourinchas, Olivier Jeanne, Patrick Kehoe, Guido Lorenzoni, Tomaso Monacelli, Maurice Obstfeld, Kenneth Rogoff, Robert Staiger and Jean Tirole. We thank seminar participants at Bocconi, Brown, Chicago, Columbia, CREI, Harvard, LSE, MIT, Princeton, University of Wisconsin, Wharton, Bank of England, ECB, IMF, NBER, NY Fed, SITE. We thank Andreas Schaab for superlative research assistance. 
large-scale transfer payments built into fiscal systems are interregional, not just interpersonal [...]" (pg. 47)

Countries such as the United States, which can be thought as a currency and fiscal union of regions, share federal revenues and transfers-through the unemployment insurance program, federal income and social security taxes and, in extreme cases, direct federal assistance-in a manner that provides macroeconomic stabilization across regions. The ongoing crisis in the Eurozone, where such mechanisms are lacking, is seen by many as a vindication of Kenen's fiscal integration criterion. Going forward, many policy discussions center around the construction of a fiscal union. How should a fiscal union be designed and how effective can we expect it to be?

Unfortunately, the OCA literature is couched in terms of Keynesian models that lack proper micro-foundations. As a result, the treatment of welfare is cursory. Recently, the New Open Economy Macro literature has developed open economy New Keynesian models with explicit microfoundations and applied them to currency areas (see e.g. Benigno 2004; Beetsma and Jensen 2005; Gali and Monacelli 2008; Ferrero 2009). Our goal is to revisit Kenen's idea using such a model. This allows for a rigorous treatment of optimal policy design. Indeed, we are able to deliver a complete characterization of the required transfers and of their effectiveness as a function of a small number of key characteristics of the economy.

We tackle the design of a fiscal union within a currency union as an optimal international risk sharing arrangement. We begin our analysis with the simplest possible model: a static setting with a traded good, a non-traded good and labor as in Obstfeld and Rogoff (2000). We then extend the analysis to a standard dynamic model featuring non-trivial intra-temporal trade and price adjustment dynamics that builds on Gali and Monacelli $(2005,2008)$. The key features in both settings are fixed exchange rates, price or wage stickiness, and limited openness in the form of non-traded goods or home bias. In this context, we set up and study the second-best planning problem for constrained efficient risk sharing via international transfers among countries in a currency union. ${ }^{1}$

International transfers have a dual role ex post. First, they help smooth consumption across countries. This is their usual direct microeconomic role. Second, under a fixed exchange rate, in the presence of nominal price or wage rigidities, and with non-traded goods or home bias, international transfers also have an indirect effect by influencing total spending across goods produced by different countries-a mechanism first discussed in the famous Transfer Problem debate involving Keynes (1929) and Ohlin (1929). Transfers from countries in a boom to countries in a bust improve macroeconomic stability in the currency union. We show that this dual role gives rise to an aggregate demand externality ex ante: the social benefits from international risk sharing are greater

\footnotetext{
${ }^{1}$ We follow the approach of the OCA literature by taking the existence of a currency union as an exogenous constraint and not attempting to model the reasons for its formation in the first place. In other words, we abstract from the potential benefits and focus on the costs of currency unions. We characterize to what extent these costs can be mitigated by the establishment of a fiscal union. Of course, one potential concern is that the factors leading to the formation of currency unions could influence the optimal design of fiscal unions. Unfortunately, there is no consensus among economists on the benefits of currency unions. In addition, at least in the case of the Eurozone, the adoption of the euro was part of a larger political unification project. For all these reasons we believe that treating the existence of a currency union as an exogenous constraint is a useful starting point.
} 
than what is appreciated by private economic agents, since they do not internalize these indirect macroeconomic stability effects and only value the direct microeconomic consumption smoothing role. Indeed, our main result is that even under ideal conditions with complete financial markets to support these international transfers, the competitive equilibrium without government intervention does not provide the constrained Pareto efficient level of international risk sharing. ${ }^{2}$

The constrained inefficiency of private international risk sharing can be addressed by government intervention. Indeed constrained efficient outcomes can be implemented in a number of ways. If individuals do have access to complete financial markets, then constrained efficiency can be ensured by introducing quantity restrictions or tax incentives that distort their individual portfolios choices. A second possibility is for the government to take over intergenerational risk sharing by assuming the necessary positions in financial markets itself. Equivalently, instead of using financial markets, it can arrange ex ante with other union members for state-contingent international fiscal transfers ex post. In either case, it must then also take steps to ensure that the private sector does not undo these arrangements by setting up the aforementioned quantity restrictions or tax incentives, or by employing more extreme measures such as banning financial markets.

We view the complete financial markets paradigm as a useful assumption to highlight that the constrained inefficiency of private risk sharing that we derive does not arise from inefficiencies in financial markets. However, our preferred interpretation is that financial markets are incomplete so that markets for sharing aggregate risk across countries are imperfect or nonexistent. This only strengthens the argument for building a fiscal union to share risks across members within a currency union. ${ }^{3}$ Indeed, the constrained efficient risk sharing arrangement can then be implemented through ex-post international fiscal transfers that are contingent on the shocks experienced by each country. Since agents have no access to financial markets, neither restrictions nor taxes on private portfolios are needed. Under this interpretation, our paper can be seen as offering a precise characterization of these ex-post international fiscal transfers and clarifying that for members of a currency union: (i) the value of gaining access to any given level of international risk sharing is greater; and (ii) international fiscal transfers should go beyond emulating the outcome that private risk sharing would reach if financial markets were complete. These two points are distinct but complement each other to motivate the formation of fiscal unions within currency unions.

Importantly, we do not reach the same conclusion for countries outside a currency union with flexible exchange rates. As long as they exercise their independent monetary policy optimally, it is optimal to let agents trade freely in a complete set of financial markets or to replicate this outcome through international fiscal transfers. Our argument for government involvement in international risk sharing relies on membership in a currency union precisely because this constrains monetary policy and prevents the stabilization of asymmetric shocks: fiscal and currency unions go hand in

\footnotetext{
${ }^{2}$ These aggregate demand externalities are pervasive in New-Keynesian models and their normative implications have recently been analyzed in different contexts in parallel and independent work by Farhi and Werning (2012), Farhi and Werning (2016), Korinek and Simsek (2016), and Schmitt-Grohe and Uribe (2016).

${ }^{3}$ Atkeson and Bayoumi (1993) examine cross-regional insurance in the United States and conclude that "integrated capital markets are [...] unlikely to provide a substantial degree of insurance against regional economic fluctuations [...] This task will continue to be primarily the business of government."
} 
hand.

Our results qualify a view often presented in the OCA literature that international fiscal transfers and international risk sharing through private financial markets are substitutes since both buffer against asymmetric macroeconomic shocks in a currency union. For example, Mundell (1973) argues that a common currency could help improve international risk sharing by increasing cross holdings of assets or deepening financial markets. While our model is silent on whether a currency union may facilitate the development of financial markets, it shows that the benefits of risk sharing are larger in a currency union and that government intervention is needed to reap the full benefits. Indeed, we establish that private risk sharing is not constrained Pareto efficient in a currency union, so that financial integration alone is not sufficient.

We emphasize three key determinants of the macroeconomic stabilization performance of fiscal unions in currency unions: the asymmetry of the shocks hitting the members of the currency union, the persistence of these shocks, and the openness of the member economies. Indeed, symmetric shocks can be accommodated with union-wide monetary policy so that transfers should be used only in response to asymmetric shocks. Optimal transfers are increasing in the persistence of these shocks but hump-shaped as a function of openness. However, a given transfer is more effective at stabilizing the economy when the economy is more closed. Hence more stabilization is achieved at the optimum both when the economy is more closed and when shocks are more persistent. Indeed, we show that full stabilization is achieved in the limit as shocks become permanent and the economy becomes closed. This contrasts with the ideas in McKinnon (1963), who discusses reasons why openness may mitigate the costs of currency unions.

We extend the model by introducing agent heterogeneity with a fraction of hand-to-mouth consumers and a fraction of permanent-income consumers. This extension allows us to capture a key feature of the data-the high average marginal propensity to consume out of transitory income and its heterogeneity across the population-while preserving enough tractability to lend itself to a fullfledged normative analysis. With hand-to-mouth consumers, the performance of transfers in dealing with transitory shocks is improved. This is because in contrast to the consumption spending of permanent-income consumers, the consumption spending of hand-to-mouth consumers tracks international fiscal transfers and hence can be efficiently targeted over time to stabilize the economy. For example in the case of a transitory recessive shock, international fiscal transfers can be front-loaded to provide stimulus in the short run when it is needed.

We compare international fiscal transfers with other macroeconomic stabilization instruments in currency unions, such as capital controls (Farhi and Werning 2012; Schmitt-Grohe and Uribe 2012) and different variants of domestic fiscal policy ranging from government spending (as analyzed in Beetsma and Jensen 2005; Gali and Monacelli 2008; Ferrero 2009; Farhi and Werning 2012, 2017) to redistribution and budget deficits. Ideally, it is best to jointly use all these different instruments together, to the extent that they are available, and our main results about the optimal design of fiscal unions are robust to the inclusion of these other instruments. But one might also want to assess the relative performance of these instruments. To that end, we discuss theoretically and compare numer- 
ically the relative performance of these different instruments depending on a number of important parameters of the economy such as the fraction of hand-to-mouth consumers, the openness of the economy, the rigidity of prices, and the persistence of shocks.

Finally, and although this is not our main focus, we briefly explore the robustness of our results in the presence of agency problems at the national level, such as limited commitment or moral hazard. We show how these incentive problems, together with the forces that we have identified above, jointly influence the optimal design of fiscal unions, and that our main insights carry over.

The rest of the paper is organized as follows. The static model is covered in Sections 2 and 3. The dynamic model is developed in Sections 4 and 5. The numerical illustrations are covered in Section 6. Section 7 contains our conclusions. The online appendix contains all the proofs and derivations as well as several extensions.

Related literature. First and foremost, our paper is related to the Optimal Currency Area (OCA) literature. This literature has emphasized a number of important factors for successful currency unions: factor mobility (Mundell, 1961), openness (McKinnon, 1963), fiscal integration (Kenen, 1969), and financial integration (Mundell, 1973). Our paper formalizes and refines the arguments of Kenen (1969), by seeing fiscal unions as the implementation of an optimal risk sharing arrangement within in a currency union, in a model with explicit micro-foundations. We offer a precise characterization of the size, direction, and effectiveness of fiscal transfers. Our results qualify the view implicit in Mundell (1973) that financial integration is a substitute for fiscal integration. Finally, our work contrasts with the ideas in McKinnon (1963), who discusses reasons why openness may mitigate the costs of currency unions. In our paper, fiscal unions are more effective when member countries are more closed. However, our results are fully compatible with the notion that openness is beneficial in a currency union lacking a fiscal union.

Our modeling approach follows the New Keynesian tradition embraced by the New Open Economy Macro literature. In particular, our static analysis builds on the model of Obstfeld and Rogoff (2000), and our dynamic analysis builds on the model of Gali and Monacelli $(2005,2008)$. A flexible exchange rate allows the implementation of the flexible price allocation (see e.g. Benigno, 2000; Clarida et al., 2002; Gali and Monacelli, 2005). A fixed exchange rate represents a constraint on macroeconomic stabilization, and raises the question of the optimal use of monetary policy in a currency union. Benigno (2004) analyzes the case of a currency union with complete markets, shows that monetary policy at the union level cannot achieve perfect stabilization with asymmetric shocks, and characterizes optimal monetary policy at the union level.

Our paper explores the optimal use of macroeconomic instruments beyond monetary policy, focusing, in particular, on cross-country transfers or interventions in financial markets. Other studies have focused on different policy instruments. Beetsma and Jensen (2005) and Gali and Monacelli (2008) analyze optimal fiscal policy in a currency union by characterizing how government purchases of domestic goods can help stabilize the economy in response to asymmetric shocks. Adao et al. (2009) and Farhi et al. (2014) show that with a rich enough set of distortionary taxes, the flexible 
price allocation can be achieved. ${ }^{4}$ In our view, however, there are important practical limitations that constrain the extent to which these tax incentives can be used, leaving considerable room for other instruments. Ferrero (2009) analyzes another dimension of fiscal policy, focusing on distortionary taxes and government debt. Farhi and Werning (2012), Schmitt-Grohe and Uribe (2012), and Farhi and Werning (2016) analyze capital controls. None of these papers considers fiscal transfers across union members and most assume complete private financial markets. Our work complements these contributions by analyzing fiscal transfers as another macroeconomic tool.

Few papers consider optimal policy with incomplete financial markets. An exception is Benigno (2009) who analyzes optimal monetary policy in the case of incomplete markets and flexible exchange rates. Nominal rigidities create a tradeoff between completing markets and stabilizing the economy. On the one hand, if prices were flexible, the optimum would imitate complete markets by tailoring the real returns of international bonds. On the other hand, if markets could be completed or if transfers imitated complete markets, the optimum would be fully efficient. Our modeling assumptions and results are essentially the polar opposite. Our analysis assumes that the exchange rate is fixed, so that the aforementioned tradeoff is not considered. Furthermore, in the presence of non-traded goods or home bias, our main result is that complete markets, or transfers that imitate complete markets, lead to a suboptimal outcome. ${ }^{5}$

Auray and Eyquem (2013) consider a currency union with sticky prices. They do not attempt to study optimal policy but using a set of numerical calibrations, they show that it is possible in some cases for the laissez faire complete markets equilibrium with a full set state-contingent bonds and no government interventions in financial markets to deliver lower welfare than the incomplete markets laissez faire equilibrium with only non-state-contingent bonds or with no bonds. This possibility is also present in our model because of the market failure that opens up a wedge between privately and socially optimal portfolios. ${ }^{6}$

The key ingredient of the New Open Economy Macro literature is the presence of nominal rigidities. Another important ingredient, present in some but not all papers in that literature, is the as-

\footnotetext{
${ }^{4}$ Kehoe and Pastorino (2017) builds on our environment but include such flexible instruments in the form of flexible country-specific and state-contingent taxes on non-traded goods, ensuring that the first best can be achieved (as if exchange rates were flexible) without transfers if markets are complete, that optimal transfers simply replicate the complete markets allocation if markets are incomplete, and also negating the need for any other macroeconomic stabilization instrument such as government spending or capital controls.

${ }^{5}$ Other features could lead to a similar result even in the absence of home bias or non-traded goods. For example, in the dynamic model of Sections 4-5, when prices are sticky but not fully rigid, complete markets, or transfers that imitate complete markets are suboptimal. This is because even in the absence of home bias, transfers still influence the domestic wage through a wealth effect, and hence also influence inflation and therefore macroeconomic stabilization. This effect, which arises only when prices are sticky but not fully rigid, would not be internalized by private agents when they form their portfolios under complete markets. This justifies interventions in financial markets if markets are complete, or transfers that do not replicate complete markets if markets are incomplete. See Section 6 and in particular footnote 38 for a discussion.

${ }^{6}$ This result, while interesting, is neither our main focus nor our main contribution, which is to study: (i) the socially optimal use of fiscal transfers to share risk between countries when markets are incomplete; (ii) the socially optimal use of state-contingent bonds to share risk between countries when markets are complete with optimal government interventions in financial markets; (iii) how effective these transfers may be in mitigating the inefficiencies from nominal rigidities and fixed exchange rates. We fully characterize how to optimally design a fiscal union within a monetary union decentralized as in (i) or as in (ii), and to understand how effective such fiscal unions are as in (iii).
} 
sumption of home bias or non-traded goods. This ingredient is absolutely central for our theory, and it is also at the core of all analyses of the Transfer Problem. Given these ingredients, we study a policy instrument that has not been considered before in the literature.

\section{A Static Model of a Currency Union}

We start with a simple static model that illustrates our main idea most transparently. Later we show that the same effects are present in standard dynamic open economy models. The model builds on the model with traded and non-traded goods presented in Obstfeld and Rogoff (2000). There is a continuum of countries in a currency union. There is a traded good, a non-traded good and labor. The traded good is supplied inelastically and traded competitively. The non-traded good is supplied from labor by monopolistic firms. The prices set by these monopolistic firms are sticky. ${ }^{7}$

We offer two market settings and associated policy interventions for the same model environment. The first assumes complete financial markets and features portfolio taxes as the policy instrument to influence equilibrium risk sharing across countries. The second assumes incomplete markets, so that private agents have no opportunities to share risk internationally. In this case we focus on government arranged international fiscal transfers across countries to provide international risk sharing. Importantly, we show that both settings lead to the same set of implementable allocations. This allows us to characterize efficient allocations using the same second-best Ramsey planning problems for both settings in Section 3.

In our view, the first setting offers several conceptual advantages even though is it less realistic. First, it allows us to make the point that constrained efficient allocations require government intervention even if financial markets are complete. By implication if markets are incomplete, government intervention should not simply mimic the complete-markets outcome. Second, we can provide simple formulas for the interventions in the form of portfolio taxes. The incomplete markets setting, on the other hand, seems more realistic and the implementation of constrained efficient allocations involves cross-country risk sharing through international fiscal transfers, providing a foundation for fiscal unions. In any case, although we favor the incomplete-market setting and its implementation in practical terms, the characterization using complete markets sheds light on both.

We first present the model with complete markets in Sections 2.1-2.4. We then introduce the incomplete markets in Section 2.5.

\subsection{Households}

There is a single period and a continuum of countries indexed by $i \in[0,1]$. We start by assuming that all countries belong to a currency union, but will relax this later. Uncertainty affects preferences and technology: the state of the world $s \in S$ has density $\pi(s)$ and determines preferences and technology,

\footnotetext{
${ }^{7}$ In Appendix B.1, we show that all our results go through if wages are nominally rigid instead of prices. In particular, Propositions 1-12 are still valid.
} 
possibly asymmetrically, in all countries.

In each country $i \in I$, there is a representative agent with preferences over non-traded goods, traded goods and labor given by the expected utility

$$
\int U^{i}\left(C_{N T}^{i}(s), C_{T}^{i}(s), N^{i}(s) ; s\right) \pi(s) d s .
$$

Below we make some further assumptions on preferences.

Agents can trade in a complete set of financial markets before the realization of the state of the world $s \in S$. Households are subject to the following budget constraints

$$
\begin{gathered}
\int D^{i}(s) Q(s) \pi(s) d s \leq 0, \\
P_{N T}^{i} C_{N T}^{i}(s)+P_{T}(s) C_{T}^{i}(s) \leq W^{i}(s) N^{i}(s)+P_{T}(s) E_{T}^{i}(s)+\Pi^{i}(s)+T^{i}(s)+\left(1+\tau_{D}^{i}(s)\right) D^{i}(s),
\end{gathered}
$$

where $P_{N T}^{i}$ is the price of non-traded goods which as we will see shortly, does not depend on $s$ due to the assumed price stickiness; $P_{T}(s)$ is the price of traded goods in state $s ; W^{i}(s)$ is the nominal wage in state $s ; E_{T}^{i}(s)$ is country $i^{\prime}$ s endowment of traded goods in state $s ; \Pi^{i}(s)$ represents aggregate profits in state $s ; T^{i}(s)$ is a lump sum rebate; $D^{i}(s)$ is the nominal payoff of the household portfolio in state $s ; Q(s)$ is the price of one unit of currency in state $s$ in world markets, normalized by the probability of state $s$; and $\tau_{D}^{i}(s)$ is a state-contingent portfolio return subsidy. ${ }^{8}$ The lump sum rebate $T^{i}(s)$ is used to rebate the proceeds from the tax on financial transactions to households. We sometimes also consider lump-sum transfers over and above such rebates to redistribute wealth across countries. Note that the nominal price of traded goods is assumed to be the same across countries, reflecting the law of one price and the fact that all countries in the union share the same currency.

The households' first-order conditions can be written as

$$
\begin{aligned}
\frac{U_{C_{T}}^{i}(s)\left[1+\tau_{D}^{i}(s)\right]}{Q(s) P_{T}(s)} & =\frac{U_{C_{T}}^{i}\left(s^{\prime}\right)\left[1+\tau_{D}^{i}\left(s^{\prime}\right)\right]}{Q\left(s^{\prime}\right) P_{T}\left(s^{\prime}\right)}, \\
\frac{U_{C_{T}}^{i}(s)}{P_{T}(s)} & =\frac{U_{C_{N T}}^{i}(s)}{P_{N T}^{i}}, \\
-\frac{U_{N}^{i}(s)}{W^{i}(s)} & =\frac{U_{C_{N T}}^{i}(s)}{P_{N T}^{i}} .
\end{aligned}
$$

\subsection{Firms}

We assume that the traded good is in inelastic supply: each country is endowed with a quantity $E_{T}^{i}(s)$ of traded goods. These goods are traded competitively in international markets.

Non-traded goods are produced in each country by competitive firms that combine a continuum

\footnotetext{
${ }^{8}$ Above we assumed that the returns from firms are not subsidized. Another possibility is to subsidize profits $\Pi^{i}(s)$ at the same rate $\tau_{D}^{i}(s)$ as financial returns. None of our analysis or conclusions are affected by this modeling choice.
} 
of non-traded varieties indexed by $j \in[0,1]$ using the constant returns to scale CES technology

$$
Y_{N T}^{i}(s)=\left(\int_{0}^{1} Y_{N T}^{i, j}(s)^{1-\frac{1}{\varepsilon}} d j\right)^{\frac{1}{1-\frac{1}{\varepsilon}}}
$$

with elasticity $\varepsilon>1$.

Each variety is produced by a monopolist using a linear technology

$$
Y_{N T}^{i, j}(s)=A^{i}(s) N^{i, j}(s)
$$

Each monopolist hires labor in a competitive market with wage $W^{i}(s)$, but pays $W^{i}(s)\left(1+\tau_{L}^{i}\right)$ net of a country specific tax on labor. Monopolists must set prices in advance, at the beginning of the period, before the realization of uncertainty. The demand for each variety is given by $C_{N T}^{i}(s)\left(P_{N T}^{i, j} / P_{N T}^{i}\right)^{-\varepsilon}$ where $P_{N T}^{i}=\left(\int\left(P_{N T}^{i, j}\right)^{1-\varepsilon} d j\right)^{1 /(1-\varepsilon)}$ is the price of non-traded goods. They solve

$$
\max _{P_{N T}^{i, j}} \int \frac{Q(s)}{1+\tau_{D}^{i}(s)} \Pi^{i, j}(s) \pi(s) d s,
$$

where

$$
\Pi^{i, j}(s)=\left[P_{N T}^{i, j}-\frac{1+\tau_{L}^{i}}{A^{i}(s)} W^{i}(s)\right] C_{N T}^{i}(s)\left(\frac{P_{N T}^{i, j}}{P_{N T}^{i}}\right)^{-\varepsilon} .
$$

Aggregate profits are given by $\Pi^{i}(s)=\int \Pi^{i, j}(s) d j$.

In a symmetric equilibrium, all monopolists in country $i$ set the same profit maximizing price. Rearranging the first-order condition yields the familiar expression for the price as a markup over a weighted average of the marginal cost across states

$$
P_{N T}^{i}=\left(1+\tau_{L}^{i}\right) \frac{\varepsilon}{\varepsilon-1} \frac{\int \frac{Q(s)}{1+\tau_{D}^{i}(s)} \frac{W^{i}(s)}{A^{i}(s)} C_{N T}^{i}(s) \pi(s) d s}{\int \frac{Q(s)}{1+\tau_{D}^{i}(s)} C_{N T}^{i}(s) \pi(s) d s} .
$$

\subsection{Government}

The government is subject to the budget constraint

$$
T^{i}(s)=\tau_{L}^{i} W^{i}(s) N^{i}(s)-\tau_{D}^{i}(s) D^{i}(s)+\hat{T}^{i}(s)
$$

Here $\hat{T}^{i}(s)$ are net international fiscal transfers which redistribute resources across countries subject to the constraint

$$
\int \hat{T}^{i}(s) d i=0,
$$

for all $s \in S$. 


\subsection{Equilibrium with Complete Markets}

An equilibrium is such that households and firms maximize, the government's budget constraint is satisfied, and markets clear?

$$
\begin{aligned}
C_{N T}^{i}(s) & =A^{i}(s) N^{i}(s), \\
\int C_{T}^{i}(s) d i & =\int E_{T}^{i}(s) d i .
\end{aligned}
$$

These conditions imply that the financial markets clear so that $\int D^{i}(s) d i=0$ for all $s \in S$.

The conditions for an equilibrium (1)-(10) act as constraints on the planning problem we study next in Section $3 .^{10}$ In a spirit similar to Lucas and Stokey (1983), we seek to drop variables and constraints as follows. Given quantities, equations (3), (5) and (6) can be used to back out certain prices, wages and taxes. Since these variables do not enter the welfare function they can be dispensed with from our planning problem, along with equations (1), (2), (3), (5), (6), (7), and (8). We summarize these arguments in the following proposition.

Proposition 1 (Implementability, Complete Markets). An allocation $\left\{C_{T}^{i}(s), C_{N T}^{i}(s), N^{i}(s)\right\}$ together with prices $\left\{P_{T}(s), P_{N T}^{i}\right\}$ form part of an equilibrium with complete markets if and only if equations (4) and (9) hold for all $i \in I, s \in S$ and equation (10) holds for all $s \in S$.

Importantly, we cannot dispense with equation (4). This equation summarizes the restriction imposed by a currency union-that the price of traded goods cannot vary across countries-and price stickiness - that the price of non-traded goods cannot vary across states of the world. Consider attempting to use equation (4) as a residual to back out the prices that support a given allocation, as we did with equations (3), (5) and (6). Equation (4) requires that the relative price of traded to non-traded goods equal $U_{C_{T}}^{i}(s) / U_{C_{N T}}^{i}(s)$. For any arbitrary allocation, this required relative price can be computed, but the problem is that it may not be possible to express it as a ratio of a price that is independent of $i$ and a price that is independent of $s$, i.e. as a ratio $P_{T}(s) / P_{N T}^{i}$. This is why we must keep equation (4) as a constraint.

Our constructive proof shows that an allocation $\left\{C_{T}^{i}(s), C_{N T}^{i}(s), N^{i}(s)\right\}$ and prices $\left\{P_{T}(s), P_{N T}^{i}\right\}$ that satisfy the conditions in the propositions are actually part of several equilibria. We emphasize two dimensions of indeterminacy. First, we can choose any set of state prices $Q(s)$. Second, we can choose different transfers $\hat{T}^{i}(s)$. These two dimensions are actually related in the sense that different state prices require different ex-post fiscal transfers.

The first dimension of indeterminacy can be intuitively understood as follows. The relevant state prices for households are adjusted for portfolio taxes $\frac{Q(s)}{1+\tau_{D}^{i}(s)}$. Scaling up state prices $Q(s)$ and the corresponding portfolio taxes $1+\tau_{D}^{i}(s)$ by a function $\lambda(s)$ leaves these tax-adjusted state prices

\footnotetext{
${ }^{9}$ Our notation already takes into account the symmetry of prices, output, and labor across varieties $j$ within each country $i$.

${ }^{10}$ In addition, the budget constraints (1) and (2) must hold as an equality.
} 
unchanged. However this change indirectly transfers resources across countries and states. These indirect transfers need to be compensated by adjusting transfers $\hat{T}^{i}(s)$.

The second dimension of indeterminacy can be intuitively understood as follows. To what extent transfers across countries actually operate through financial markets $D^{i}(s)$ or transfers $\hat{T}^{i}(s)$ is not pinned down. This can easily be seen by starting with the household's budget constraint, holding with equality, and substituting out profits $\Pi^{i}(s)$ and transfers $T^{i}(s)$ to arrive at the following country budget constraint

$$
\int Q(s)\left[P_{T}(s)\left(C_{T}^{i}(s)-E_{T}^{i}(s)\right)\right] \pi(s)=\int Q(s) \hat{T}^{i}(s) \pi(s) d s,
$$

which states that the expected value of the trade balance must be covered by the expected value of international fiscal transfers. Indeed, this is the only constraint on fiscal transfers. For example, one possibility is to constrain transfers to be non-state-contingent $\hat{T}^{i}(s)=\hat{T}^{i}$. All risk sharing is then being delivered through financial markets, and portfolio taxes are required to make sure that private agents secure the right amount of insurance $D^{i}(s)$. Another possibility is to set $\hat{T}^{i}(s)=$ $P_{T}(s)\left(C_{T}^{i}(s)-E_{T}^{i}(s)\right)$. In that case, all risk sharing is being delivered through transfers. Portfolio taxes are then required to ensure that private agents do not "undo" these transfers.

\subsection{Equilibrium with Incomplete Markets}

We also consider an alternative setup where markets are incomplete, in the sense that there are no financial markets before the realization of the state of the world $s \in S$. We split the representative agent in country $i$ into a continuum of households $j \in[0,1]$. Household $j$ is assumed to own the firm of variety $j$. Household $j$ maximizes utility

$$
\int U^{i}\left(C_{N T}^{i}(s), C_{T}^{i}(s), N^{i}(s) ; s\right) \pi(s) d s,
$$

by choosing $\left\{C_{T}^{i}(s), C_{N T}^{i}(s), N^{i}(s)\right\}$ and the prices set by its own firm $P_{N T}^{i, j}$, taking aggregate prices and wages $\left\{P_{T}(s), P_{N T}^{i}, W^{i}(s)\right\}$ and aggregate demand $\left\{\bar{C}_{N T}^{i}(s)\right\}$ as given, subject to

$$
P_{N T}^{i} C_{N T}^{i}(s)+P_{T}(s) C_{T}^{i}(s) \leq W^{i}(s) N^{i}(s)+P_{T}(s) E_{T}^{i}(s)+\Pi^{i, j}(s)+T^{i}(s),
$$

where

$$
\Pi^{i, j}(s)=\left[P_{N T}^{i, j}-\frac{1+\tau_{L}^{i}}{A^{i}(s)} W^{i}(s)\right] \bar{C}_{N T}^{i}(s)\left(\frac{P_{N T}^{i, j}}{P_{N T}^{i}}\right)^{-\varepsilon} .
$$


The corresponding first-order conditions are symmetric across $j$ and given by (4) and (5) and the price setting condition

$$
P_{N T}^{i}=\left(1+\tau_{L}^{i}\right) \frac{\varepsilon}{\varepsilon-1} \frac{\int \frac{U_{C_{T}}^{i}(s)}{P_{T}(s)} \frac{W^{i}(s)}{A^{i}(s)} \bar{C}_{N T}^{i}(s) \pi(s) d s}{\int \frac{U_{C_{T}}^{i}(s)}{P_{T}(s)} \bar{C}_{N T}^{i}(s) \pi(s) d s} .
$$

In equilibrium we impose the consistency condition that $\bar{C}_{N T}^{i}(s)=C_{N T}^{i}(s)$ for all $i$ and $s$.

The government budget constraint simplifies to

$$
T^{i}(s)=\tau_{L}^{i} W^{i}(s) N^{i}(s)+\hat{T}^{i}(s) .
$$

We can now define an equilibrium with incomplete markets. An equilibrium specifies quantities $\left\{C_{T}^{i}(s), C_{N T}^{i}(s), N^{i}(s)\right\}$, prices and wages $\left\{P_{T}(s), P_{N T}^{i}, w^{i}(s)\right\}$, taxes $\left\{\tau_{L}^{i}, T^{i}(s)\right\}$ and international fiscal transfers $\left\{\hat{T}^{i}(s)\right\}$ such that households and firms maximize, the government's budget constraint is satisfied, and markets clear. More formally, the conditions for an equilibrium are given by (4), (5), (8), (11) holding with equality, (12) with $\bar{C}^{i}(s)=C^{i}(s)$, and (13).

As in the complete markets implementation, we can drop variables and constraints as follows. Given quantities, equations (5) and (12) can be used to back out certain prices, wages and taxes. Since these variables do not enter the welfare function they can be dispensed with from our planning problem, along with equations (5), (8), (11), (12), and (13). We summarize these arguments in the following proposition.

Proposition 2 (Implementability, Incomplete Markets). An allocation $\left\{C_{T}^{i}(s), C_{N T}^{i}(s), N^{i}(s)\right\}$ together with prices $\left\{P_{T}(s), P_{N T}^{i}\right\}$ form part of an equilibrium with incomplete markets if and only if equations (4) and (9) hold for all $i \in I, s \in S$ and equation (10) holds for all $s \in S$.

Propositions 1 and 2 reach the same implementability conditions for the complete- and incompletemarket settings. Although the set of implementable quantities $\left\{C_{T}^{i}(s), C_{N T}^{i}(s), N^{i}(s)\right\}$ and prices $\left\{P_{T}(s), P_{N T}^{i}\right\}$ is the same, the required policy instruments are of course different.

Under complete markets, portfolio taxes $\left\{\tau_{D}^{i}(s)\right\}$ are needed, and transfers $\left\{\hat{T}^{i}(s)\right\}$ are largely indeterminate. In contrast, in the incomplete market setting no restriction on private portfolios are introduced since no assets are available to private agents. In this case, transfers $\left\{\hat{T}^{i}(s)\right\}$ are uniquely determined as $\hat{T}^{i}(s)=P_{T}(s)\left(C_{T}^{i}(s)-E_{T}^{i}(s)\right)$ and are typically state-contingent.

\subsection{Homothetic Preferences}

We characterize the key condition (4) further by making some weak assumptions on preferences: (i) preferences over consumption goods are weakly separable from labor; and (ii) preferences over consumption goods are homothetic. Denoting by $p^{i}(s)=\frac{P_{T}(s)}{P_{N T}^{i}}$ the relative price of traded goods in 
state $s$ in country $i$, these assumptions imply that

$$
C_{N T}^{i}(s)=\alpha^{i}\left(p^{i}(s) ; s\right) C_{T}^{i}(s),
$$

for some function $\alpha^{i}(p ; s)$ that is increasing and differentiable in its first argument. This conveniently encapsulates the restriction implied by the first-order condition (4). This condition is crucial because the stickiness of non-traded prices, together with the lack of monetary independence, places restrictions on the possible variability across $i \in I$, for any state of the world $s$, in the relative price $p^{i}(s)$.

\section{Constrained Efficient Risk Sharing in the Static Model}

In this section we characterize constrained Pareto efficient allocations. Before doing so, it is useful to briefly describe first-best allocations. They are characterized by two requirements. First, the marginal rate of substitution between labor and non-traded goods must be equal to the corresponding marginal rate of transformation ${ }^{11}$

$$
\frac{-U_{N}^{i}(s)}{U_{C_{N T}}^{i}(s)}=A^{i}(s)
$$

for every sate $s$ and country $i$. Second, the standard optimal risk-sharing conditions

$$
\frac{U_{C_{T}}^{i}(s)}{U_{C_{T}}^{i}\left(s^{\prime}\right)}=\frac{U_{C_{T}}^{i^{\prime}}(s)}{U_{C_{T}}^{i^{\prime}}\left(s^{\prime}\right)},
$$

must hold for every pair of states $\left(s, s^{\prime}\right)$ and countries $\left(i, i^{\prime}\right)$. For every first-best allocation, we can find Pareto weights $\lambda^{i}$ such that $U_{C_{T}}^{i}(s)=\frac{\lambda^{i}}{\lambda^{\prime}} U_{C_{T}}^{i^{\prime}}(s)$ for every state $s$ and pair of countries $\left(i, i^{\prime}\right)$ and conversely, to any set of Pareto weights $\lambda^{i}$ corresponds a first-best allocation, so that we can think of the set of first-best allocations as being indexed by these Pareto weights.

As we shall see, first-best allocations are typically not achievable in a currency union with nominal rigidities under our competitive equilibrium notion with or without complete markets. Instead we seek to characterize second best allocations that are constrained Pareto efficient among the allocations that are the outcomes of a competitive equilibrium. For this purpose, it is useful to first introduce the labor wedge

$$
\tau^{i}(s)=1+\frac{1}{A^{i}(s)} \frac{U_{N}^{i}(s)}{U_{C_{N T}}^{i}(s)}
$$

for every state $s$ and country $i$. Labor wedges are zero at first-best allocations, but are typically not in equilibrium allocations. The optimal risk sharing condition above holds at allocations which are the outcomes of competitive equilibria with complete markets when there are no interventions in financial markets. They typically do not hold at allocations which are the outcomes of competitive

\footnotetext{
${ }^{11}$ In this and other expressions and functions we streamline the notation by leaving the dependence on some of the arguments implicit.
} 
equilibria with complete markets when there are interventions in financial markets, or which are the outcome of competitive equilibria with incomplete markets. A fundamental result of our analysis below is that they typically do not hold at second-best allocations.

\subsection{Indirect Utility Function for Transfers}

Define the indirect utility function

$$
V^{i}\left(C_{T}, p ; s\right)=U^{i}\left(\alpha^{i}(p ; s) C_{T}, C_{T}, \frac{\alpha^{i}(p ; s)}{A^{i}(s)} C_{T} ; s\right)
$$

In an equilibrium with $C_{T}^{i}(s)$ and $p^{i}(s)$, ex-post welfare in state $s$ in country $i$ is then given by $V^{i}\left(C_{T}^{i}(s), p^{i}(s) ; s\right)$. The derivatives of the indirect utility function will prove useful for our analysis.

Proposition 3. The derivatives of the indirect utility function are

$$
V_{p}^{i}(s)=\frac{\alpha_{p}^{i}(s)}{p^{i}(s)} C_{T}^{i}(s) U_{C_{T}}^{i}(s) \tau^{i}(s) \quad \text { and } \quad V_{C_{T}}^{i}(s)=U_{C_{T}}^{i}(s)\left[1+\frac{\alpha^{i}(s)}{p^{i}(s)} \tau^{i}(s)\right]
$$

These observations about the derivatives and their connection to the labor wedge will be key to our results. A private agent values a transfer in traded goods according to its private marginal utility $U_{C_{T}}^{i}(s)$, but the actual social marginal value in equilibrium is $V_{C_{T}}^{i}(s)$. The wedge between the two equals $\frac{\alpha^{i}(s)}{p^{i}(s)} \tau^{i}(s)=\frac{P_{N T}^{i} C_{N T}(s)}{P_{T}(s) C_{T}(s)} \tau^{i}(s)$, the labor wedge weighted by the relative expenditure share of non-traded goods relative to traded goods. We will sometimes refer to it as the weighted labor wedge for short.

In particular, a private agent undervalues transfers $V_{C_{T}}^{i}(s)>U_{C_{T}}^{i}(s)$ whenever the economy is experiencing a recession, in the sense of having a positive labor wedge $\tau^{i}(s)>0$. Conversely, private agents overvalue the costs of making transfers $V_{C_{T}}^{i}(s)<U_{C_{T}}^{i}(s)$ whenever the economy is booming, in the sense of having a negative labor wedge $\tau^{i}(s)<0$. These effects are magnified when the economy is relatively closed, so that the relative expenditure share of non-traded goods is large. ${ }^{12}$

When country $i$ receives a transfer, its consumers feel richer and increase their spending on both traded and non-traded goods in equal proportions. Since prices are fixed, the resulting increased demand for non-traded goods translates one-for-one into an increase in output. This in turn generates more income, further raising spending etc. This mechanism is at the core of the famous Transfer Problem controversy between Keynes (1929) and Ohlin (1929). These equilibrium effects, which are not internalized by private agents, open up a wedge between the social and private marginal values of transfers.

\footnotetext{
${ }^{12}$ Note that this definition of boom and bust is anchored in the difference between output and the potential level of output, in the sense that a boom is defined to be a situation where output is above potential. These Keynesian notions of boom and bust are the relevant ones for the allocation of transfers across countries. It is perfectly possible for output to be below potential but for potential output to be high, so that the country could be growing and experiencing a bust at the same time.
} 
Since the increase in demand for both goods is proportional, the "dollar-for-dollar" output multiplier of transfers is precisely given by the relative expenditure share of non-traded to traded goods $\frac{P_{N T}^{i} C_{N T}^{i}(s)}{P_{T}(s) C_{T}^{i}(s)}$. The labor wedge $\tau^{i}(s)$ summarizes the net calculation for utility of the increase in nontraded consumption and the increase in labor that accompany the increase in output. This explains why the wedge between the social and private marginal valuations is precisely $\frac{P_{N T}^{i} C_{N T}^{i}(s)}{P_{T}(s) C_{T}^{i}(s)} \tau^{i}(s) .{ }^{13}$

\subsection{Second-Best Ramsey Planning Problem}

We consider a planning problem that allows us to characterize the set of constrained Pareto efficient allocations. The planning problem is indexed by a set of nonnegative Pareto weights $\lambda^{i}$. By varying these Pareto weights, we can trace out the entire constrained Pareto frontier. The second-best planning problem is

$$
\max _{P_{T}(s), P_{N T}^{i}, C_{T}^{i}(s)} \iint \lambda^{i} V^{i}\left(C_{T}^{i}(s), \frac{P_{T}(s)}{P_{N T}^{i}} ; s\right) \pi(s) d i d s
$$

subject to

$$
\int C_{T}^{i}(s) d i=\int E_{T}^{i}(s) d i .
$$

Let $\mu(s) \pi(s)$ be the multiplier on the resource constraint in state $s \in S$. The first-order conditions for $C_{T}^{i}(s), P_{T}(s)$ and $P_{N T}^{i}$ are respectively

$$
\begin{aligned}
\lambda^{i} V_{C_{T}}^{i}(s) & =\mu(s), \\
\int V_{p}^{i}(s) \frac{1}{P_{N T}^{i}} \lambda^{i} d i & =0, \\
\int V_{p}^{i}(s) p^{i}(s) \pi(s) d s & =0 .
\end{aligned}
$$

These first-order conditions tightly characterize the solution. The first-order condition for $P_{N T}^{i}$ implies our first proposition.

Proposition 4 (Optimal Price Setting). At a constrained Pareto efficient equilibrium, for every country i, a weighted average of labor wedges across states is zero

$$
\int \alpha_{p}^{i}(s) C_{T}^{i}(s) U_{C_{T}}^{i}(s) \tau^{i}(s) \pi(s) d s=0 .
$$

In the absence of uncertainty this proposition implies a zero labor wedge in each state. With uncertainty, in general the labor wedge for a given country takes on both signs across states with a

\footnotetext{
${ }^{13}$ It is theoretically possible for the marginal value of a transfer to be negative $V_{C_{T}}^{i}(s)<0$ if the labor wedge is sufficiently negative, especially if the share of non-traded goods, relative to traded goods, is large enough. In this extreme case a country can improve welfare by making gift transfers, without any counterpart transfer in the opposite direction. If $\tau^{i}$ is sufficiently negative then unilateral gift transfers to other countries are welfare enhancing for country $i$. This extreme case will not be our focus and is not employed in any of our results below. However, it is a stark example of just how divergent public and private valuations of transfers can become.
} 
weighted average of zero. ${ }^{14}$

The first-order condition for $P_{T}(s)$ implies the following proposition.

Proposition 5 (Optimal Monetary Policy). At a constrained Pareto efficient equilibrium, in every state s, a weighted average of labor wedges across countries is zero

$$
\int \alpha_{p}^{i}(s) C_{T}^{i}(s) U_{C_{T}}^{i}(s) \tau^{i}(s) \lambda^{i} d i=0
$$

This proposition establishes that optimal monetary policy targets a weighted average across countries for the labor wedge. It sets this target to zero in each state of the world. The intuition for the result is that monetary policy can be chosen at the union level, and can adapt across states to the average condition. If all countries are identical and the shock is symmetric, then we obtain perfect stabilization in each country: $\tau^{i}(s)=0$ for all $i \in I, s \in S$. By contrast, when shocks across countries are not symmetric then perfect stabilization is impossible. However, at the union level the economy is stabilized in the sense that the weighted average for the labor wedge across countries is set to zero for all states of the world $s \in S .^{15}$

Finally, the first-order condition for $C_{T}(s)$ says that the marginal utility of transfers in traded goods adjusted for the Pareto weight $\lambda^{i} V_{C_{T}}^{i}(s)$ should be equalized across countries for every state $s$, or in other words that for every pair of states $\left(s, s^{\prime}\right)$, and pair of countries $\left(i, i^{\prime}\right)$, we have

$$
\frac{V_{C_{T}}^{i}(s)}{V_{C_{T}}^{i}\left(s^{\prime}\right)}=\frac{V_{C_{T}}^{i^{\prime}}(s)}{V_{C_{T}}^{i^{\prime}}\left(s^{\prime}\right)} .
$$

It is more revealing to rewrite this condition using our expressions for the derivative of $V_{C_{T}}^{i}(s)$.

Proposition 6 (Constrained Efficient Risk Sharing). At a constrained Pareto efficient equilibrium, for every pair of states $\left(s, s^{\prime}\right)$, and pair of countries $\left(i, i^{\prime}\right)$, risk sharing takes the following form

$$
\frac{U_{C_{T}}^{i}(s)\left[1+\frac{\alpha^{i}(s)}{p^{i}(s)} \tau^{i}(s)\right]}{U_{C_{T}}^{i}\left(s^{\prime}\right)\left[1+\frac{\alpha^{i}\left(s^{\prime}\right)}{p^{i}\left(s^{\prime}\right)} \tau^{i}\left(s^{\prime}\right)\right]}=\frac{U_{C_{T}}^{i^{\prime}}(s)\left[1+\frac{i^{i^{\prime}}(s)}{p^{i^{\prime}}(s)} \tau^{i^{\prime}}(s)\right]}{U_{C_{T}}^{i^{\prime}}\left(s^{\prime}\right)\left[1+\frac{\alpha^{i^{\prime}}\left(s^{\prime}\right)}{p^{i^{\prime}}\left(s^{\prime}\right)} \tau^{i^{\prime}}\left(s^{\prime}\right)\right]} .
$$

If markets are complete and portfolio taxes are not employed, then the risk sharing condition (3) imposes the additional constraint that for every pair of states $\left(s, s^{\prime}\right)$ and countries $\left(i, i^{\prime}\right)$, we have

$$
\frac{U_{C_{T}}^{i}(s)}{U_{C_{T}}^{i}\left(s^{\prime}\right)}=\frac{U_{C_{T}}^{i^{\prime}}(s)}{U_{C_{T}}^{i^{\prime}}\left(s^{\prime}\right)} .
$$

\footnotetext{
${ }^{14}$ In the absence of uncertainty, the labor tax to cancel the monopolistic markup $\tau_{L}^{i}=-1 / \varepsilon$. With uncertainty, in general $\tau_{L}^{i} \neq-1 / \varepsilon$. When the sub-utility function between $C_{N T}$ and $C_{T}$ is a CES so that $\alpha(\cdot ; s)$ has constant elasticity, independent of $s$, then $\tau_{L}^{i}=-1 / \varepsilon$ is optimal even with uncertainty. The proof is contained in the Appendix A.3.

${ }^{15}$ The result is related to the result in Benigno (2004) and Gali and Monacelli (2008) that optimal monetary policy in a currency union ensures that the union average output gap, in a linearized version of the model, is zero in every period. Here the result is obtained without linearizing the model and it is expressed in terms of the labor wedge, instead of the output gap.
} 
Comparing these conditions, one may expect the private risk sharing condition (16) to be incompatible with the constrained efficient risk sharing condition (15) except in special cases. Indeed, we next show that because labor wedges must average to zero across states and countries according to Propositions 4 and 5, they are indeed incompatible unless the first best is attainable. This implies that equilibria with privately optimal risk sharing are constrained Pareto inefficient. ${ }^{16}$

Proposition 7 (Inefficiency of Private Risk Sharing). An equilibrium with complete markets and no portfolio taxes $\left(\tau_{D}^{i}(s)=0\right.$ for all $\left.i \in I, s \in S\right)$ is constrained Pareto inefficient unless $\tau^{i}(s)=0$ for all $i \in I, s \in S$, in which case it is first best.

With complete markets but without interventions in financial markets, private agents do not secure the constrained efficient amount of risk sharing in financial markets. They do not fully internalize the macroeconomic stability consequences of their portfolio decisions, opening a role for government intervention in financial markets. ${ }^{17}$

\subsection{Implementation}

We now turn to the implementation of constrained Pareto efficient allocations. With complete markets, constrained Pareto efficient equilibria can be decentralized with appropriate labor taxes $\tau_{L}^{i}$ and corrective portfolio taxes $\tau_{D}^{i}(s)$. Proposition 6 leads to a neat characterization of the required taxes.

Proposition 8 (Complete Markets and Portfolio Taxes). If private asset markets are complete, constrained Pareto efficient allocations can be implemented by the following portfolio return subsidy/taxes

$$
\tau_{D}^{i}(s)=\frac{\alpha^{i}(s)}{p^{i}(s)} \tau^{i}(s)
$$

Insurance for bad states of the world, where the weighted labor wedge is high, should be relatively subsidized. Government intervention secures additional transfers from low weighted labor wedge countries ("boom" countries) to high weighted labor wedge countries ("bust" countries). This reduces the demand for non-traded goods in the boom countries and increases it in the bust countries, stabilizing output and income. These stabilization benefits are not internalized by private agents, hence the need for government intervention.

It is interesting to note that the taxes do not depend directly on the Pareto weights $\lambda^{i}$, but only indirectly through the relative expenditure share of non-traded goods and the labor wedge. This underscores the fact that they are imposed to correct a macroeconomic aggregate demand externality and not to redistribute.

\footnotetext{
${ }^{16}$ Note that our definition of constrained efficient risk sharing is a second best concept which is different from the concept of first best risk sharing. At the first best, both the constrained efficient risk sharing condition (15) and the privately optimal risk sharing condition (16) hold, because the labor wedges are all equal to zero. Away from the first best, these two conditions differ. Another way of stating our result is that it is optimal to deviate from the target of first-best risk sharing in order to help achieve the other target of stabilizing the economy.

${ }^{17}$ We should also point out that the Propositions 5 and 6 go through if non-traded goods prices are entirely predetermined (i.e. are exogenously fixed).
} 
The implementation of the social optimum with corrective portfolio taxes is only one interesting possibility. Another equally interesting interpretation of our results assumes that private asset markets are nonexistent, so that private opportunities for risk sharing are unavailable. The same optimum can then be implemented through state-contingent transfers. ${ }^{18}$

Proposition 9 (Incomplete Markets and Ex-Post Transfers). If private asset markets are incomplete so that state-contingent assets are unavailable, constrained Pareto efficient allocations can also be implemented through state-contingent transfers

$$
\hat{T}^{i}(s)=P_{T}(s)\left(C_{T}^{i}(s)-E^{i}(s)\right)
$$

Under this alternative implementation, no restriction on private portfolios are needed since no assets are available to private agents. Our results can then be seen as offering a precise characterization of the required transfers. A key conclusion of our analysis is that these transfers would go beyond replicating the outcome that private risk sharing decisions would achieve if markets were complete.

It is also possible to imagine implementations that are in between the two polar cases of corrective portfolio taxes with complete markets and state-contingent transfers with incomplete markets. In general, government positions in asset markets, or state-contingent transfers, combined with restrictions or tax incentives on private portfolios are required.

It is important to stress that our results hold for any constrained Pareto efficient allocation, or equivalently, for any set of Pareto weights $\lambda^{i}$. They are not driven by a desire to redistribute across countries and instead reflect an insurance motive. One way to see this is that in general it is always possible to find Pareto weights $\lambda^{i}$ such that $\int \hat{T}^{i}(s) \pi(s) d s=0$ for every country $i$. For example, in the special case where countries are ex-ante identical these Pareto weights are equal $\lambda^{i}=\lambda^{i^{\prime}}$ for all pair of countries $\left(i, i^{\prime}\right)$. Our results hold for this particular set of Pareto weights. Except in knife edge circumstances where the corresponding allocation is first best, portfolio taxes will be required for its implementation if markets are complete, and transfers will go beyond replicating complete markets if markets are incomplete.

\footnotetext{
${ }^{18}$ We could also envision intermediate cases of incomplete markets, in which case both portfolio taxes and transfers will be required in conjunction. In these cases, transfers are useful and required to the extent that they expand risksharing possibilities beyond those available through financial markets, and portfolio taxes are required to influence risk-sharing through financial markets. Assume for simplicity that $S$ is discrete that there are $N$ aggregate states of nature, and that there the space $\mathcal{A}$ of asset payoffs that is available through traded securities is of dimension $M \leq N$. Then the decentralization requires both portfolio taxes and fiscal transfers. In general, to implement the optimum in that way requires access to a set state-contingent fiscal transfers $\mathcal{F}$ such that $\mathcal{A}+\mathcal{F}=\mathcal{R}^{N}$. This implies that $\operatorname{dim}(\mathcal{F})=N-$ $\operatorname{dim}(\mathcal{A})-\operatorname{dim}(A \cap \mathcal{F}) \geq N-M$. If this is not the case, then in some cases, the constrained Pareto efficient allocations that we have characterized cannot be decentralized, which means that we need to set up a different, more constrained, planning problem.
} 


\subsection{Countries Outside the Currency Union}

Up to this point we have assumed that all countries belong to the currency union. Now, imagine that only a subset of countries $I \subseteq[0,1]$ are members. The rest manage monetary policy independently as follows. Country $i \notin I$ sets its own local nominal price for the traded good $P_{T}^{i}(s)=E^{i}(s) P_{T}(s)$ in its home currency by manipulating the level of its exchange rate $E^{i}(s)$ against the union's currency. ${ }^{19}$ The planning problem becomes

$$
\max \int_{i \in I} \lambda^{i} V^{i}\left(C_{T}^{i}(s), \frac{P_{T}(s)}{P_{N T}^{i}} ; s\right) d i+\int_{i \notin I} \lambda^{i} V^{i}\left(C_{T}^{i}(s), \frac{P_{T}^{i}(s)}{P_{N T}^{i}} ; s\right) d i
$$

subject to

$$
\int C_{T}^{i}(s) d i=\int E_{T}^{i}(s) d i .
$$

For a country $i \notin I$ outside the union, the first-order condition for $P_{T}^{i}(s)$ is

$$
V_{p}^{i}\left(C_{T}^{i}(s), p^{i}(s) ; s\right)=0
$$

Since $V_{p}^{i}\left(C_{T}^{i}(s), p^{i}(s) ; s\right)=\frac{\alpha_{p}^{i}(s)}{p^{i}(s)} C_{T}^{i}(s) U_{C_{T}}^{i}(s) \tau^{i}(s)$, it follows that

$$
\tau^{i}(s)=0 \quad \text { for all } s \in S, i \notin I .
$$

A flexible exchange rate leads to perfect stabilization, in the sense that the labor wedge is set to zero for all states of the world. This result is reminiscent of the arguments set forth by Friedman (1953) and Mundell (1961) in favor of flexible exchange rates. For countries in the currency union, optimal monetary policy is still imperfect and characterized by the average condition for the labor wedge in Proposition 5.

The constrained efficient risk sharing condition in Proposition 6 still applies to all countries, inside or outside the currency union. However, since $\tau^{i}(s)=0$ for $s \in S, i \notin I$, this condition coincides with the privately optimal risk sharing condition for countries outside the currency union. As a result, there is no need to upset private risk sharing.

Proposition 10 (Countries Outside the Currency Union). None of the results are affected by considering countries outside the union. Countries that have independent monetary policy manage to obtain a zero labor wedge $\tau^{i}(s)=0$. If markets are complete, they should not intervene in financial markets $\tau_{D}^{i}(s)=0$. If markets are incomplete, they should seek to secure ex-post transfers $\hat{T}^{i}(s)$ that replicate privately optimal risk sharing outcomes.

If markets are incomplete, then transfers might be required even outside a currency union. Interestingly, we will show that, in the dynamic version of the model with only traded goods and

\footnotetext{
${ }^{19}$ Since the price of traded goods is modeled as flexible here, we do not require assumptions about Producer Currency Pricing (PCP) versus Local Currency Pricing (LCP).
} 
home bias in preferences, there are cases (the Cole-Obstfeld case) where transfers are not required for countries outside a currency union, whereas they are required for countries inside a currency union.

Crucially, our results establish that inside a currency union, transfers should go beyond replicating the outcome that would arise if markets were complete. In this sense, our results yield two important insights. First, currency unions and fiscal unions go hand in hand. Second, fiscal integration and financial integration are not perfect substitutes.

How are attitudes towards risk affected by membership in a union? We show that members are more risk averse in the following sense. Suppose country $i$ belongs to the currency union with equilibrium relative price $p^{i}(s)$. The advantage of leaving the union is that the relative price $p^{i}$ is not constrained and welfare attains the first best level conditional on $C_{T}^{i}$. Define $V^{i *}\left(C_{T}^{i} ; s\right)=$ $\max _{p} V^{i}\left(C_{T}^{i}, p ; s\right)$. It follows that

$$
V^{i}\left(C_{T}^{i}, p^{i}(s) ; s\right) \leq V^{i *}\left(C_{T}^{i} ; s\right)
$$

with equality if and only if $p^{i}(s) \in \arg \max _{p} V^{i}\left(C_{T}^{i}, p ; s\right)$, in which case the labor wedge is zero, $\tau(s)=0$. Thus, for every state $s$, the function $V^{i *}$ is the upper envelope over $V^{i}\left(C_{T}^{i}, p ; s\right)$ for different values of $p$ and is tangent to it precisely at a level of $p$ that implies a zero labor wedge. In this sense, $V^{i}\left(C_{T}^{i}, p^{i}(s) ; s\right)$ is more concave than $V^{i *}$ and member countries are more risk averse. We shall put this inequality to use in the next section.

\subsection{Value of Risk Sharing}

Our simple model allows for three random disturbances: (i) shocks to productivity of labor in the production of non-traded goods; (ii) shocks to preferences (demand); and (iii) shocks to the endowment of traded goods. Proposition 7 shows that if the equilibrium with complete markets but without portfolio taxes does not attain the first best, then it is constrained inefficient. As we show next, this is the case except in knife-edge cases. Examining these knife-edge cases turns out to be interesting, because even when the equilibria coincide with the first best we find that the planner values the availability of insurance strictly more than private agents do, and do in that sense, risk sharing is of greater social than private value. Extrapolating beyond our model, this could help explain why macro insurance markets may be missing, even if their social value is significant.

To engineer an example where the first best is attainable it is useful to specialize the model by using the utility function

$$
U^{i}\left(C_{T}, C_{N T}, N ; s\right)=\log \left(C_{T}\right)+\alpha^{i}(s) \log \left(C_{N T}\right)-\frac{1}{1+\phi} N^{1+\phi},
$$

with $\phi \geq 0$.

Proposition 11. Suppose that the utility function is given by (19), then the equilibrium with complete markets but without portfolio taxes is constrained efficient if and only if it is first best, which occurs if and only if 
productivity shocks and preference shocks are such for all pairs of countries $\left(i, i^{\prime}\right)$, the ratio

$$
\frac{A^{i}(s)}{A^{i^{\prime}}(s)}\left(\frac{\alpha^{i}(s)}{\alpha^{i^{\prime}}(s)}\right)^{\frac{-\phi}{1+\phi}}
$$

is constant for all $s \in S$.

The key constraint imposed by nominal rigidities and a single monetary policy is condition (4), rewritten here for convenience as

$$
\frac{U_{C_{N T}}^{i}(s)}{U_{C_{T}}^{i}(s)}=\frac{P_{N T}^{i}}{P_{T}(s)}
$$

where $P_{T}(s)$ is only allowed to vary with $s$ not $i$, while $P_{N T}^{i}$ is allowed to vary with $i$ but not $s$. One can handle fixed differences across countries and union-wide shocks to this marginal rate of substitution, but not individual variations.

Proposition 11 defines a precise notion of symmetric shocks to productivity and preferences for which the first best allocation is attainable without portfolio taxes. For example, if the only shocks are to productivity, then this condition requires that productivity vary proportionally across countries. A currency union can handle such a shock using union-wide monetary policy. A similar point applies to taste shocks.

This discussion highlights just how special these circumstances are. Note, however, that the proposition implies that traded good endowment shocks can be properly insured with complete markets and without portfolio taxes. To understand this result, suppose we only have shocks to the endowment of traded goods. Then the first best features perfect risk sharing in the consumption of traded goods so that only aggregate fluctuations in the endowment of traded goods affect the consumption of traded goods. Due to the separability of preferences, the first best allocation for non-traded goods and labor is not affected by these shocks. It follows that the marginal rate of substitution only varies with union-wide shocks, and the first best is implementable as an equilibrium..$^{20,21}$

It is useful to have a case, however artificial, where private risk sharing is constrained efficient so that we can isolate a separate result. We show that members of a currency union value this risk sharing more than countries outside it. Moreover, this is is not true of the value placed on risk sharing by private individuals. This highlights the role of the aggregate demand externality from risk sharing decisions, which is not internalized by private agents.

${ }^{20}$ In more detail, suppose $A^{i}(s)=A^{i}$ and $\alpha^{i}(s)=\alpha^{i}$. The first best allocation features $C_{T}^{i}(s)=\frac{1}{\lambda^{i}} \int_{0}^{1} E^{i}(s) d i, N^{i}(s)=$ $\left(\alpha^{i}\right)^{\frac{1}{1+\phi}}$, and $C_{N T}^{i}(s)=A^{i}\left(\alpha^{i}\right)^{\frac{1}{1+\phi}}$. This allocation is supported as an equilibrium without portfolio taxes by $P_{N T}^{i}=$ $\left(\alpha^{i}\right)^{\frac{\phi}{1+\phi}} /\left(\lambda^{i} A^{i}\right), P_{T}(s)=\left(\int_{0}^{1} E^{i}(s) d i\right)^{-1}, W^{i}(s)=\left(\alpha^{i}\right)^{\frac{\phi}{1+\phi}} / \lambda^{i}, Q(s)=1$ and $1+\tau_{L}^{i}=\frac{\varepsilon-1}{\varepsilon}$.

${ }^{21}$ Of course, the case of traded good endowment shocks is somewhat artificial, relying on the modeling asymmetry that non-traded goods are produced but traded goods are not. If instead traded goods were produced from labor and another fixed input (capital or land) subject to (industry specific) productivity shocks, then these shocks would also have to satisfy the restriction of being symmetric to attain the first best-just as in the case of productivity shocks in the non-traded goods. 


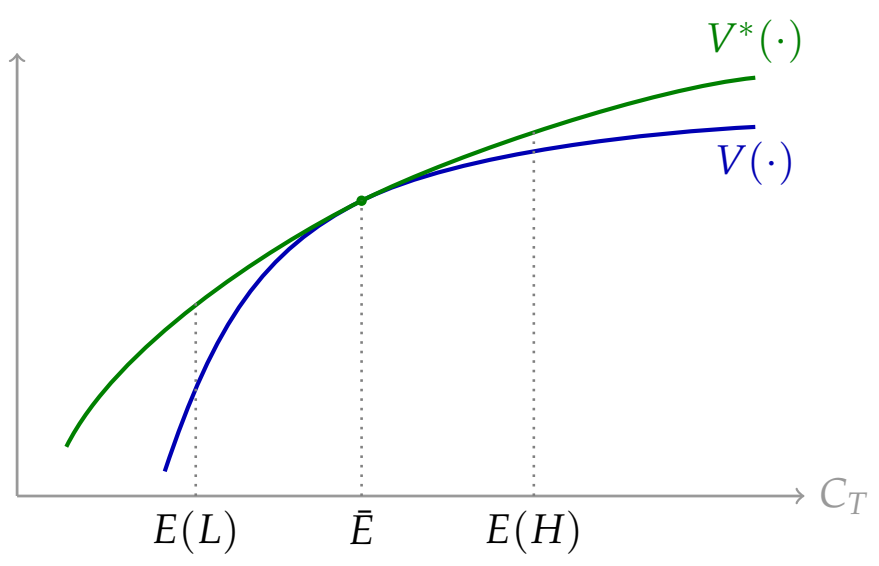

Figure 1: Welfare as perceived by individual agents (upper green curve) and country as a whole (lower blue curve).

Proposition 12. Suppose that there are only traded good endowment shocks and that all risk is idiosyncratic, so that the aggregate endowment of traded goods is constant across states. If we exclude a country from risk sharing, then its utility loss is greater if it belongs to a currency union. If we exclude a single individual within a country from risk sharing, then the utility loss is the same whether or not his country belongs to a currency union.

Figure 1 illustrates the basic logic behind the first part this proposition for an example with two equiprobable endowment values. Since the aggregate endowment is constant, the price of traded goods is constant and complete markets without portfolio taxes offer fair insurance. The resulting equilibrium features constant consumption of the traded good at the average value of the endowment and constant prices and wages. This is true for both members and non members. When the country is excluded from risk sharing its consumption of the traded good must now fluctuate with its endowment, creating a mean-preserving spread in consumption of traded goods and a loss in expected utility. The crucial point is that the loss is greater for union members because they are more risk averse, according to inequality (18). Indeed, given that prices are constant and the utility function is independent of the state $s$ this inequality simplifies to $V^{i}\left(C_{T}^{i}, \bar{p}\right) \leq \max _{p} V^{i}\left(C_{T}^{i}, p\right)=V^{i *}\left(C_{T}^{i}\right)$. These two indirect utility functions are depicted in the figure. They are tangent at the average value of the endowment $\bar{E}$ because this represents the equilibrium consumption level with risk sharing.

As to the second part of the proposition, it follows easily from the observation that in the context of this specific example, the equilibrium with risk sharing is the same whether or not the country belongs to the currency union. In both cases the first best allocation is attained. Therefore, if an individual is excluded from risk sharing, he faces the same prices whether the country is a member or not. Thus, the drop in utility is the same. 


\subsection{Insurance vs. Incentives: Limited Commitment and Moral Hazard}

Explicit or implicit insurance arrangements inevitably raise concerns of incentives. We have abstracted from these considerations, not because we believe them to be unimportant, but in order to isolate the effects of the aggregate demand externality on optimal risk sharing. Modeling them requires making specific choices regarding the underlying agency problems.The possibilities are vast and exploring them all is beyond the scope of this paper, but we believe that the main insights of our analysis would carry over. ${ }^{22}$

In the appendix, we analyze two examples, one where countries have a limited ability to commit to paying international transfers to other countries (Appendix B.2) and one where countries can engage in a form of moral hazard which influences the distribution of states of the world (Appendix B.3). In both cases, there is a meaningful tradeoff between insurance and incentives, and the optimality condition characterizing the optimal resolution of this tradeoff involves the social marginal utility of transfers, a key sufficient statistic which is different for countries in a currency union where it is given by $U_{C_{T}}^{i}(s)\left[1+\frac{\alpha^{i}(s)}{p^{i}(s)} \tau^{i}(s)\right]$ than for countries with a flexible exchange rate where it is given by $U_{C_{T}}^{i}(s)$.

\section{A Dynamic Model}

The static model reveals some key results in a simple and transparent manner. However, it is perhaps too simple to explore the issues in greater depth, and in particular to think about two key determinants of fiscal unions: price adjustment dynamics and the persistence of shocks. We now build a richer, dynamic model similar to Farhi and Werning (2012) which in turn builds on Gali and Monacelli $(2005,2008)$. We present the model with incomplete markets where agents can only trade short-term risk free bonds as in Farhi and Werning (2012) ${ }^{23}$ Just as in the static model, we tackle the optimal design of international state-contingent fiscal transfers.

In Section 5, we analyze the model using a log-linear approximation of the nonlinear model around its perfect foresight symmetric steady state. We derive an impulse response characterization of optimal transfers following a shock. It is well understood that in log-linearized models, agents behave according to a certainty equivalence principle, and that the impulse response to a particular shock is independent of the probability distribution of shocks in the nonlinear model. For these reasons, and to simplify the exposition, we focus our presentation of the nonlinear model in this section on the perfect foresight case where one-time unanticipated shocks to the path of productivity

\footnotetext{
${ }^{22}$ In practice of course, institutional mechanisms exist to mitigate these agency problems. For example, most fiscal unions such as the US channel a large part of their transfers through more or less rules-based automatic stabilizers (through the unemployment insurance program, federal income and social security taxes, bailout funds), probably for reasons of political acceptability and transparency, but also to mitigate the difficulties associated with collecting and distributing discretionary transfers ex post. Other examples are state debt limits in the US, and collective budget procedures and enforcement mechanisms that already exist in Europe.

${ }^{23}$ We will also compare it to the complete financial market case when we turn to the log-linearized version of the model in Section 5.
} 
in each country are realized at $t=0$, with no further uncertainty. ${ }^{24}$ The assumption of incomplete markets translates into the requirement that absent international transfers, the initial net foreign asset position of any given country be independent of the particular realization of the shock. International transfers between countries can be arranged contingent on the realization of shocks and it is precisely the optimal arrangement of these transfers in response to shocks that we seek to characterize. ${ }^{25}$

\subsection{Households}

There is a continuum of measure one of countries $i \in[0,1]$. We focus attention on a single country, which we call Home, and which can be thought of as a particular value $H \in[0,1]$. In every country, there is a representative household with preferences represented by the utility function

$$
\sum_{t=0}^{\infty} \beta^{t}\left[\frac{C_{t}^{1-\sigma}}{1-\sigma}-\frac{N_{t}^{1+\phi}}{1+\phi}\right]
$$

where $N_{t}$ is labor, and $C_{t}$ is a consumption index defined by $C_{t}=\left[(1-\alpha)^{\frac{1}{\eta}} C_{H, t}^{\frac{\eta-1}{\eta}}+\alpha^{\frac{1}{\eta}} C_{F, t}^{\frac{\eta-1}{\eta}}\right]^{\frac{\eta}{\eta-1}}$. Here $C_{H, t}$ is an index of consumption of domestic goods given by $C_{H, t}=\left(\int_{0}^{1} C_{H, t}(j)^{\frac{\epsilon-1}{\epsilon}} d j\right)^{\frac{\epsilon}{\epsilon-1}}$, where $j \in$ $[0,1]$ denotes an individual good variety. Similarly, $C_{F, t}$ is a consumption index of imported goods given by $C_{F, t}=\left(\int_{0}^{1} C_{i, t}^{\frac{\gamma-1}{\gamma}} d i\right)^{\frac{\gamma}{\gamma-1}}$, where $C_{i, t}$ is, in turn, an index of the consumption of varieties of goods imported from country $i$, given by $C_{i, t}=\left(\int_{0}^{1} C_{i, t}(j)^{\frac{\epsilon-1}{\epsilon}} d j\right)^{\frac{\epsilon}{\epsilon-1}}$.

Thus $\epsilon$ is the elasticity between varieties produced within a given country, $\eta$ the elasticity between domestic and foreign goods, and $\gamma$ the elasticity between goods produced in different foreign countries. An important special case is the Cole-Obstfeld case which obtains when $\sigma=\eta=\gamma=1$. This case, which was first studied by Cole and Obstfeld (1991), is more tractable and has some special implications which we will highlight later in Sections (5)-(6).

The parameter $\alpha$ indexes the degree of home bias and can be interpreted as a measure of openness. As $\alpha$ approaches zero, the share of foreign goods vanishes. As $\alpha$ approaches one, the share of home goods vanishes. Since the country is infinitesimal, the latter captures a very open economy without home bias, while the former captures a closed economy barely trading with the outside world.

\footnotetext{
${ }^{24}$ Consider a fully stochastic version of the nonlinear model where shocks are drawn from a given distribution in every period starting at $t=0$. Take the point-wise limit when the variance of the distribution of shocks goes to zero of the impulse response to a given shock of the log-linearized version of this model. It coincides with the impulse response of the log-linearized version of the perfect foresight model to a one-time unanticipated shock. Because markets are incomplete, the performance of the log-linear approximation of the perfect foresight model as an approximation of the fully stochastic version of the nonlinear deteriorates in the long run as the economy moves away from the steady state in response to successive shocks, but because of discounting, this does not affect the validity of our characterization of optimal transfers and of their benefits in response to shocks at $t=0$. These issues do not arise in the perfect foresight version of the model with one-time unanticipated shocks.

${ }^{25}$ As should be clear from this description, our notion of market incompleteness is that markets are incomplete across states of the world but not over time.
} 
Households seek to maximize their utility subject to the sequence of budget constraints

$$
\begin{aligned}
\int_{0}^{1} P_{H, t}(j) C_{H, t}(j) d j+\int_{0}^{1} \int_{0}^{1} P_{i, t}(j) C_{i, t}(j) & d j d i+D_{t+1}+\int_{0}^{1} E_{i, t} D_{t+1}^{i} d i \\
\leq & W_{t} N_{t}+\Pi_{t}+T_{t}+\left(1+i_{t-1}\right) D_{t}+\int_{0}^{1} E_{i, t}\left(1+i_{t-1}^{i}\right) D_{t}^{i} d i
\end{aligned}
$$

for $t=0,1,2, \ldots$ In this inequality, $P_{H, t}(j)$ is the price of domestic variety $j, P_{i, t}(j)$ is the price of variety $j$ imported from country $i, W_{t}$ is the nominal wage, $\Pi_{t}$ represents nominal profits, and $T_{t}$ is a nominal lump sum transfer. All these variables are expressed in domestic currency. The portfolio of home agents is composed of home and foreign bond holding: $D_{t}$ is home bond holdings of home agents, $D_{t}^{i}$ is bond holdings of country $i$ of home agents. The returns on these bonds are determined by the nominal interest rate in the home country $i_{t}$, the nominal interest rate $i_{t}^{i}$ in country $i$, and the evolution of the nominal exchange rate $E_{i, t}$ between Home and country $i$.

\subsection{Firms}

Technology. A typical firm in the home economy produces a differentiated good with a linear technology given by

$$
Y_{t}(j)=A_{H, t} N_{t}(j),
$$

where $A_{H, t}$ is productivity in the home country. We denote productivity in country $i$ by $A_{i, t}$.

We allow for a constant employment tax $1+\tau^{L}$, so that real marginal cost deflated by Home PPI is given by

$$
M C_{t}=\frac{1+\tau^{L}}{A_{H, t}} \frac{W_{t}}{P_{H, t}} .
$$

We take this employment tax to be constant in our model. We pin down this tax rate by assuming that it is set optimally and cooperatively set at a symmetric steady state with flexible prices. The tax rate is simply set to offset the monopoly distortion so that $\tau^{L}=-\frac{1}{\varepsilon}$.

Price-setting assumptions. As in Gali and Monacelli (2005), we maintain the assumption that the Law of One Price (LOP) holds so that at all times, the price of a given variety in different countries is identical once expressed in the same currency. This assumption is known as Producer Currency Pricing (PCP) and is sometimes contrasted with the assumption of Local Currency Pricing (LCP), where each variety's price is set separately for each country and quoted (and potentially sticky) in that country's local currency. Thus, LOP does not necessarily hold. It has been shown by Devereux and Engel (2003) that LCP and PCP may have different implications for monetary policy. However, for our purposes, these two polar cases are equivalent since, for the most part, we will study the model assuming fixed exchange rates.

We consider Calvo price setting, where in every period, a randomly selected fraction $1-\delta$ of 
firms can reset their prices. Those firms that get to reset their price choose a reset price $P_{t}^{r}$ to solve

$$
\max _{P_{t}^{r}} \sum_{k=0}^{\infty} \delta^{k}\left(\prod_{h=1}^{k} \frac{1}{1+i_{t+h}}\right)\left(P_{t}^{r} Y_{t+k \mid t}-P_{H, t} M C_{t} Y_{t+k \mid t}\right)
$$

where $Y_{t+k \mid t}=\left(\frac{P_{t}^{r}}{P_{H, t+k}}\right)^{-\epsilon} C_{t+k}$, taking the sequences for $M C_{t}, Y_{t}$, and $P_{H, t}$ as given.

\subsection{Government}

The government is subject to the budget constraint

$$
T_{t}=\tau_{L} W_{t} N_{t}+\hat{T}_{t}
$$

Here $\hat{T}_{t}$ are net international fiscal transfers. The transfers $\hat{T}_{t}$ are the focus of our analysis. More precisely, we allow for international fiscal transfers across countries, contingent on the shocks experienced by these countries.

\subsection{Terms of Trade and Real Exchange Rate}

It is useful to define the following price indices: home's Consumer Price Index (CPI) $P_{t}=[(1-$ a) $\left.P_{H, t}^{1-\eta}+\alpha P_{F, t}^{1-\eta}\right]^{\frac{1}{1-\eta}}$, home's Producer Price Index (PPI) $P_{H, t}=\left[\int_{0}^{1} P_{H, t}(j)^{1-\epsilon} d j\right]^{\frac{1}{1-\epsilon}}$, and the index for imported goods $P_{F, t}=\left[\int_{0}^{1} P_{i, t}^{1-\gamma} d i\right]^{\frac{1}{1-\gamma}}$, where $P_{i, t}=\left[\int_{0}^{1} P_{i, t}(j)^{1-\epsilon} d j\right]^{\frac{1}{1-\epsilon}}$ is country $i^{\prime}$ s PPI.

Let $E_{i, t}$ be the nominal exchange rate between home and $i$ (an increase in $E_{i, t}$ is a depreciation of the home currency). Because the Law of One Price holds, we can write $P_{i, t}(j)=E_{i, t} P_{i, t}^{i}(j)$ where $P_{i, t}^{i}(j)$ is country $i$ 's price of variety $j$ expressed in its own currency. Similarly, $P_{i, t}=E_{i, t} P_{i, t}^{i}$ where $P_{i, t}^{i}=\left[\int_{0}^{1} P_{i, t}^{i}(j)^{1-\epsilon}\right]^{\frac{1}{1-\epsilon}}$ is country $i^{\prime}$ s domestic PPI in terms of country $i$ 's own currency. We therefore have $P_{F, t}=E_{t} P_{t}^{*}$, where $P_{t}^{*}=\left[\int_{0}^{1} P_{i, t}^{i 1-\gamma} d i\right]^{\frac{1}{1-\gamma}}$ is the world price index and $E_{t}$ is the effective nominal exchange rate. ${ }^{26}$

The effective terms of trade are defined by

$$
S_{t}=\frac{P_{F, t}}{P_{H, t}}=\left(\int_{0}^{1} S_{i, t}^{1-\gamma} d i\right)^{\frac{1}{1-\gamma}}
$$

where $S_{i, t}=P_{i, t} / P_{H, t}$ is the terms of trade of home versus $i$. The terms of trade can be used to rewrite the home CPI as $P_{t}=P_{H, t}\left[1-\alpha+\alpha S_{t}^{1-\eta}\right]^{\frac{1}{1-\eta}}$.

Finally we can define the real exchange rate between home and $i$ as $\mathcal{Q}_{i, t}=E_{i, t} P_{t}^{i} / P_{t}$ where $P_{t}^{i}$ is country'i's CPI. We define the effective real exchange rate to be

$$
\mathcal{Q}_{t}=\frac{E_{t} P_{t}^{*}}{P_{t}}
$$

\footnotetext{
${ }^{26}$ The effective nominal exchange rate is defined as $E_{t}=\left[\int_{0}^{1} E_{i, t}^{1-\gamma} P_{i, t}^{i 1-\gamma} d i\right]^{\frac{1}{1-\gamma}} /\left[\int_{0}^{1} P_{i, t}^{i 1-\gamma} d i\right]^{\frac{1}{1-\gamma}}$.
} 


\subsection{Equilibrium Conditions}

We now summarize the equilibrium conditions. Equilibrium in the home country can be described by the following equations. We find it convenient to group these equations into two blocks, which we refer to as the demand block and the supply block.

The demand block is independent of the nature of price setting. It is composed of the BackusSmith condition

$$
C_{t}=\Theta^{i} C_{t}^{i} \mathcal{Q}_{i, t^{\prime}}^{\frac{1}{\sigma}}
$$

where $\Theta^{i}$ is a relative Pareto weight which depends on the realization of the shocks, the goods market clearing condition

$$
Y_{t}=\left(\frac{P_{H, t}}{P_{t}}\right)^{-\eta}\left[(1-\alpha) C_{t}+\alpha \int_{0}^{1} C_{t}^{i}\left(S_{t}^{i} S_{i, t}\right)^{\gamma-\eta} \mathcal{Q}_{i, t}^{\eta} d i\right]
$$

were $S_{t}^{i}$ is denotes the effective terms of trade of country $i$, the labor market clearing condition

$$
N_{t}=\frac{Y_{t}}{A_{H, t}} \Delta_{t}
$$

where $\Delta_{t}$ is an index of price dispersion $\Delta_{t}=\int_{0}^{1}\left(\frac{P_{H, t}(j)}{P_{H, t}}\right)^{-\epsilon}$, the Euler equation

$$
1+i_{t}=\beta^{-1} \frac{C_{t+1}^{\sigma}}{C_{t}^{\sigma}} \Pi_{t+1}
$$

where $\Pi_{t}=\frac{P_{t+1}}{P_{t}}$ is CPI inflation, the arbitrage condition between home and foreign bonds

$$
1+i_{t}=\left(1+i_{t}^{i}\right) \frac{E_{i, t+1}}{E_{i, t}},
$$

for all $i \in[0,1]$, and the country budget constraint

$$
\sum_{t=0}^{\infty}\left(\prod_{s=0}^{t-1} \frac{1}{1+i_{s}}\right) \hat{T}_{t}=-\sum_{t=0}^{\infty}\left(\prod_{s=0}^{t-1} \frac{1}{1+i_{s}}\right)\left(P_{H, t} Y_{t}-P_{t} C_{t}\right) .
$$

Equation (21) is central to our analysis and it is useful to unpack it. We define $N F A_{0}$ as the net present value of transfers received by Home expressed in terms of the initial home CPI

$$
N F A_{0}=\frac{1}{P_{0}} \sum_{t=0}^{\infty}\left(\prod_{s=0}^{t-1} \frac{1}{1+i_{s}}\right) \hat{T}_{t} .
$$

Equation (21) can be restated as equating the net present value of transfers to the net present value 
of trade deficits

$$
N F A_{0}=-\frac{1}{P_{0}} \sum_{t=0}^{\infty}\left(\prod_{s=0}^{t-1} \frac{1}{1+i_{s}}\right)\left(P_{H, t} Y_{t}-P_{t} C_{t}\right) .
$$

This makes clear that the net present value of transfers $N F A_{0}$ acts like a de facto like initial net foreign asset position which depends on the shock. With ex-ante identical countries, absent international fiscal transfers, we would have $N F A_{0}=0$. Characterizing the optimal value of $N F A_{0}$ depending on the shocks is one the main focuses of our analysis below. We will also compare the optimal value of $N F A_{0}$ to the value that would obtain if private agents could engage in risk sharing through a complete set of financial markets. One of our main results will establish that these values differ, and to characterize how they differ.

Finally with Calvo price setting, the supply block is composed of the equations summarizing the first-order condition for optimal price setting. These conditions are provided in Appendix C.1. We will only analyze a log-linearized version of the model with Calvo price setting (see Section 5).

For most of the paper, we will be concerned with fixed exchange rate regimes (either pegs or currency unions) in which case we have the additional restriction that $E_{t}=E_{0}$ for all $t \geq 0$ where $E_{0}$ is predetermined.

\section{Optimal Transfers in the Dynamic Model}

As is standard in the literature, we work with a log-linearized approximation of the model around a symmetric steady state where productivity (and hence output) in all countries is equal to one. As before, at $t=0$, the economy is hit with an unanticipated shock. It is convenient to work with a continuous time version of the model. This does not affect our results, but it is useful because it implies that no price index can jump at $t=0$ and this simplifies the derivation of initial conditions characterizing the equilibrium. We denote the instantaneous discount rate by $\rho$, and the instantaneous arrival rate for price changes by $\rho_{\delta}$.

From now on we focus on the Cole-Obstfeld case $\sigma=\eta=\gamma=1$. This case is attractive for the following reason. Even when prices are sticky, the allocation with incomplete markets and no transfers coincides with the equilibrium with complete markets and no interventions in financial markets. Risk sharing is delivered with balanced trade. This means that we can interpret any deviation from balanced trade at the optimum with transfers as an indication that private risk sharing through complete financial markets, if they were available, would be constrained inefficient. Third, it is possible to derive a simple second-order approximation of the welfare function around the symmetric deterministic steady state. Away from the Cole-Obstfeld case, this derivation is much more involved.

We start by considering the case where all countries are members of the same currency union. We then consider the case where some countries are in a currency union, while others remain outside, with a flexible exchange rate and independent monetary policy. 


\subsection{The Log-Linearized Economy}

We denote with lowercase variables the log deviations from the symmetric steady state of the corresponding uppercase variable introduced in Section 4 . We denote with a star the union average of a given variable. For example $\tilde{y}_{t}^{*}=\int_{0}^{1} \tilde{y}_{t}^{i} d i, \tilde{c}_{t}^{*}=\int_{0}^{1} \tilde{c}_{t}^{i} d i$, and $a_{t}^{*}=\int_{0}^{1} a_{i, t} d i$.

Shocks. The shocks hitting the model are $a_{i, t}$. They have both an aggregate component $a_{t}^{*}$ and an idiosyncratic component $a_{i, t}-a_{t}^{*}$. We can think of the aggregate component as reflecting symmetric shocks and of the idiosyncratic component as capturing asymmetric shocks.

The natural allocation. We define the natural allocation as the flexible price allocation when there are no transfers across countries. We describe this allocation in log deviations from the symmetric steady state with a lower case, and tilde.

At the natural allocation, output in country $i$ is given by $\tilde{y}_{t}^{i}=a_{i, t}$, consumption is given by $\tilde{c}_{t}^{i}=\alpha a_{t}^{*}+(1-\alpha) a_{i, t}$, labor is given by $\tilde{n}_{t}^{i}=0$, and the terms of trade are given by $\tilde{s}_{t}{ }^{i}=a_{i, t}-a_{t}^{*}$, where by construction $\int_{0}^{1} \tilde{s}_{t}^{i} d i=\int_{0}^{1} \tilde{y}_{t}^{i} d i=\int_{0}^{1} \tilde{a}_{i, t} d i=0 .{ }^{27}$ In addition, trade is balanced. Finally, aggregate output is equal to aggregate consumption and is given by $\tilde{y}_{t}^{*}=\tilde{c}_{t}^{*}=a_{t}^{*}$.

Summarizing the system in gaps. We denote by $\hat{y}_{t}^{i}$ and $\hat{\theta}^{i}$ the deviations of $y_{t}^{i}$ and $\theta^{i}$ from their natural counterparts. We further define the gaps of these variables to their respective union average as $\hat{\bar{y}}_{t}^{i}=\hat{y}_{t}^{i}-\hat{y}_{t}^{*}, \hat{\theta}^{i}=\hat{\theta}^{i}-\hat{\theta}^{*}$, and $\hat{\pi}_{H, t}^{i}=\pi_{H, t}^{i}-\pi_{t}^{*}$. We have $\int \hat{y}_{t}^{i} d i=\int \hat{\theta}^{i} d i=\int \hat{\pi}_{H, t}^{i} d i=0$. Note that $\hat{\theta}^{i}$ is already a normalized variable so that $\hat{\theta}^{*}=0$ and $\hat{\theta}^{i}=\hat{\theta}^{i}$.

The deviation of the trade balance from its natural counterpart of zero is constant and equal to $-\alpha \hat{\theta}^{i}$. The net present value of transfers must pay for the net present value of the trade deficits, so that $N \hat{\bar{F}} A_{0}^{i}=\frac{\alpha}{\rho} \hat{\theta}^{i}$.

The disaggregated variables solve two ordinary differential equations, namely the Phillips curve

$$
\dot{\bar{\pi}}_{H, t}^{i}=\rho \hat{\bar{\pi}}_{H, t}^{i}-\kappa_{y} \hat{\bar{y}}_{t}^{i}-\lambda \alpha \hat{\bar{\theta}}^{i}
$$

and the Euler equation

$$
\dot{\hat{y}}_{t}^{i}=-\hat{\pi}_{H, t}^{i}-\dot{\tilde{s}}_{t}^{i}
$$

with the initial condition

$$
\hat{\bar{y}}_{0}^{i}=(1-\alpha) \hat{\bar{\theta}}^{i}-\tilde{s}_{0}^{i},
$$

where $\lambda=\rho_{\delta}\left(\rho+\rho_{\delta}\right)$ and $\kappa_{y}=\lambda(1+\phi)$ index price flexibility. We assume that the zero lower bound on the nominal interest is not binding. Then the only constraint on the aggregates is that they must satisfy the aggregate New Keynesian Philips Curve

$$
\dot{\pi}_{t}^{*}=\rho \pi_{t}^{*}-\kappa_{y} \hat{y}_{t}^{*} .
$$

\footnotetext{
${ }^{27}$ Although we do not need it for our analysis, note that the natural interest rate is given by $\tilde{r}_{t}^{i}=\dot{a}_{i, t}$.
} 
Thus, there are many possible paths for the aggregate variables, depending on the stance of monetary policy at the union level.

From these equations we can infer aggregate consumption $\hat{c}_{t}^{*}=\hat{y}_{t}^{*}$. We can also infer the disaggregated variables for country $i$ as follows. The terms of trade gap $\hat{s}_{t}^{i}$ can be backed out from $\hat{\bar{y}}_{t}^{i}=(1-\alpha) \hat{\bar{\theta}}^{i}+\hat{\bar{s}}_{t}^{i}$, which combines the market clearing condition with the Backus-Smith condition. Similarly, we can back out the employment gap $\hat{n}_{t}^{i}$ and the consumption gap $\hat{c}_{t}^{i}$ from technology $\hat{\bar{y}}_{t}^{i}=\hat{\bar{n}}_{t}^{i}$ and market clearing $\hat{y}_{t}^{i}=\hat{\bar{c}}_{t}^{i}+\alpha \hat{\bar{s}}_{t}^{i}-\alpha \hat{\bar{\theta}}^{i}$.

Loss function. We are interested in characterizing the symmetric constrained Pareto efficient allocation that provides optimal ex-ante risk sharing behind the veil of ignorance before shocks are realized. To do so, we maximize an unweighted utilitarian welfare function. A simple representation of the loss function associated with this welfare criterion is as follows (see Farhi and Werning, 2012):

$$
\frac{(1+\phi)}{2} \int_{0}^{\infty} \int_{0}^{1} e^{-\rho t}\left[\alpha_{\pi}\left(\hat{\bar{\pi}}_{H, t}^{i}+\pi_{t}^{*}\right)^{2}+\left(\hat{\bar{y}}_{t}^{i}+\hat{y}_{t}^{*}\right)^{2}+\alpha_{\theta}\left(\hat{\bar{\theta}}^{i}\right)^{2}\right] d i d t,
$$

where $\alpha_{\pi}=\frac{\epsilon}{\lambda(1+\phi)}$ and $\alpha_{\theta}=\frac{\alpha(2-\alpha)}{1+\phi}$.

The first two terms in the loss function are familiar in New Keynesian models and are identical to those obtained by Gali and Monacelli $(2005,2008)$. The third term captures the direct welfare effects of transfers and penalizes deviations from privately optimal risk sharing. This term disappears in the closed-economy limit as $\alpha \rightarrow 0 .^{28}$

\subsection{Optimal Transfers in a Currency Union}

Before we begin the analysis of optimal transfers, it is useful to make the following observation. ${ }^{29}$ Throughout Sections 4-6, we assume that markets are incomplete. Suppose for a moment that markets are complete. Then the best equilibrium with complete markets and no intervention in financial markets has $\tilde{\theta}^{i}=0$. It coincides with the best equilibrium with incomplete markets and no transfers. ${ }^{30}$ This equivalence is due to our Cole-Obstfeld specification. As a result, we can interpret any nonzero transfer at the optimum with incomplete markets as an indication that private risk sharing through complete financial markets, if those were available, would be constrained inefficient. See Appendix C.3 for details.

We set up the planning problem for optimal transfers under incomplete markets. We use the fact that $\int_{0}^{1} \hat{\bar{y}}_{t}^{i} d i=\int_{0}^{1} \hat{\bar{\pi}}_{H, t}^{i} d i=0$. We are led to the following second-best planning problem

$$
\min \frac{1}{2} \int_{0}^{\infty} \int_{0}^{1} e^{-\rho t}\left[\alpha_{\pi}\left(\hat{\pi}_{H, t}^{i}\right)^{2}+\left(\hat{\bar{y}}_{t}^{i}\right)^{2}+\alpha_{\theta}\left(\hat{\bar{\theta}}^{i}\right)^{2}+\alpha_{\pi}\left(\pi_{t}^{*}\right)^{2}+\left(\hat{y}_{t}^{*}\right)^{2}\right] d i d t
$$

\footnotetext{
${ }^{28}$ Note that from the perspective of an individual country $i$, transfers also have a first-order effect on welfare. The loss function of an individual country inherits a term $-\frac{1}{2} \int_{0}^{\infty} e^{-\rho t} \frac{2 \alpha(2-\alpha)}{1+\phi} \hat{\theta}^{i} d t$. This term represents the pure distributional aspect of transfers. These distributional concerns are zero-sum and wash out in the aggregate since $\int_{0}^{1} \hat{\theta}^{i} d i=0$.

${ }^{29}$ In Appendix C.4, we solve for the positive effects of transfers in a currency union.

${ }^{30}$ In both cases, optimal monetary policy ensures that the aggregate output gap and inflation are zero $\hat{y}_{t}^{*}=\pi_{t}^{*}=0$.
} 
subject to

$$
\begin{gathered}
\dot{\hat{\bar{\pi}}}_{H, t}^{i}=\rho \hat{\bar{\pi}}_{H, t}^{i}-\kappa_{y} \hat{\bar{y}}_{t}^{i}-\lambda \alpha \hat{\bar{\theta}}^{i}, \\
\dot{\hat{y}}_{t}^{i}=-\hat{\bar{\pi}}_{H, t}^{i}-\dot{\tilde{s}}_{t}^{i}, \\
\hat{\bar{y}}_{0}^{i}=(1-\alpha) \hat{\bar{\theta}}^{i}-\tilde{s}_{0}^{i}, \\
\int_{0}^{1} \hat{\bar{\theta}}^{i} d i=0, \\
\dot{\pi}_{t}^{*}=\rho \pi_{t}^{*}-\kappa_{y} \hat{y}_{t}^{*},
\end{gathered}
$$

where the minimization is over the variables $\hat{\bar{\pi}}_{H, t}^{i}, \pi_{t}^{*}, \hat{\bar{y}}_{t}^{i}, \hat{y}_{t}^{*}, \hat{\bar{\theta}}^{i}$.

In Appendix C.2, we show how to decompose this planning problem into an aggregate planning problem that determines $\pi_{t}^{*}$ and $\hat{y}_{t}^{*}$, and a disaggregated planning problem that determines $\hat{\bar{\pi}}_{H, t}^{i}, \hat{\bar{y}}_{t}^{i}$, and $\hat{\bar{\theta}}^{i}$ for all $i$. Furthermore, we show that the disaggregated planning problem can be relaxed and broken down into separate component planning problems indexed by $i$. The aggregate planning problem does not depend on the shocks. The disaggregated planning problem depends only on the asymmetric component of shocks captured by the natural terms of trade $\tilde{s}_{t}^{i}=a_{i, t}-a_{t}^{*}$. The symmetric component of shocks $a_{t}^{*}$ does not explicitly show up in the planning problem expressed in gaps.

Monetary policy can be chosen at the union level so that monetary conditions are tailored to the average country, a result which echoes Proposition 5 from the static model and is reminiscent of the results in Benigno (2004) and Gali and Monacelli (2008).

Proposition 13 (Optimal Monetary Policy). At the second-best optimum, union-wide aggregates are zero $\hat{y}_{t}^{*}=\pi_{t}^{*}=0$.

This means that monetary policy at the union level can perfectly stabilize the symmetric component of shocks. Monetary policy is powerless, however, in dealing with the asymmetric component of shocks.

We now characterize disaggregated variables at the second-best optimum and study how transfers are optimally used to stabilize the asymmetric component of shocks $\tilde{s}_{t}^{i}$. We provide closed-form solutions for two enlightening special cases: rigid prices and the closed-economy limit. We then explore the general case using extensive numerical simulations in Section 6.

The case of rigid prices. We first treat the case of rigid prices $\kappa_{y}=0$.

Proposition 14 (Rigid Prices). Suppose that prices are rigid, then at the second-best optimum we have

$$
N \hat{\bar{F}} A_{0}^{i}=\frac{\alpha(1-\alpha)}{(1-\alpha)^{2}+\alpha_{\theta}} \int_{0}^{\infty} e^{-\rho t} \tilde{s}_{t}^{i} d t \quad \text { and } \quad \hat{\bar{\theta}}^{i}=\frac{\rho(1-\alpha)}{(1-\alpha)^{2}+\alpha_{\theta}} \int_{0}^{\infty} e^{-\rho t} \tilde{s}_{t}^{i} d t .
$$

Importantly, we find that the net present value of transfers $N \hat{\bar{F}} A_{0}^{i}$ and $\hat{\theta}^{i}$ and are nonzero, so that the optimal solution does not coincide with the solution that would have arisen with complete markets and no interventions in financial markets. 
Countries experiencing shocks that depreciate their natural terms of trade $\tilde{s}_{t}^{i}$ receive positive transfers. With positive home bias $(\alpha<1)$, these transfers increase the demand for home goods and help alleviate the temporary recession resulting from the inability of the terms of trade to immediately depreciate to their natural level in the short run.

Optimal transfers are increasing in the size of the shocks. They are also increasing in the persistence of the shocks. This is intuitive since consumers permanently increase their consumption in response to the transfers. As a result, the more transitory the shock, the less the increase in spending is concentrated when the economy is depressed in the short run. In other words, transfers affect the economy permanently and are therefore better suited to deal with persistent shocks.

Optimal transfers depend crucially on the openness of the economy as captured by the degree of home bias $\alpha$. They are non-monotonic in the degree of openness. Indeed, the net present value of transfers is zero for $\alpha=0$ (closed economy) and for $\alpha=1$ (fully open economy).

The reasons for zero transfers with $\alpha=0$ and with $\alpha=1$ are very different. For $\alpha$ close to 0 , transfers have large expenditure switching effects across goods from different countries and therefore large effects on output. For $\alpha$ close to 1 , transfers have small expenditure switching effects and therefore small effects on output. So for $\alpha$ close to 0 , we get small transfers because transfers are very effective, and indeed we get $\hat{\bar{\theta}}^{i} \neq 0$ so that the allocation remains significantly different from the one arising with no transfers. By contrast for $\alpha$ close to 1, we get small transfers because transfers are very ineffective, and we get $\hat{\theta}^{i}=0$ so that the allocation approaches the one prevailing with no transfers.

The case of the closed-economy limit. We now return to the case where prices are not entirely rigid, $\kappa_{y}>0$, but we place ourselves in the closed-economy limit $\alpha \rightarrow 0$.

Proposition 15 (Closed-Economy Limit). In the closed-economy limit and when $\tilde{s}_{t}^{i}=\tilde{s}_{0}^{i} e^{-\psi t}$, at the secondbest optimum we have

$$
N \hat{\bar{F}} A_{0}^{i}=0 \quad \text { and } \quad \hat{\bar{\theta}}^{i}=\tilde{s}_{0}^{i}\left[\frac{\kappa_{y}}{\kappa_{y}-(\rho+\psi) \psi}+\frac{\rho-2 v}{\alpha_{\pi} v^{2}+1} \frac{\left(\alpha_{\pi} v \kappa_{y}-\rho-\psi\right) \psi}{(\rho+\psi-v)\left[\kappa_{y}-(\rho+\psi) \psi\right]}\right]
$$

This proposition shows that the intuition given above in the case of rigid prices for the increased effectiveness of transfers as the economy becomes more closed generalizes to the case where prices are not entirely rigid. As we approach the closed-economy limit, optimal transfers become vanishingly small ( $N \hat{\bar{F}} A_{0}^{i}=0$ in the limit) but the allocation remains significantly different from the allocation with no transfers $\left(\hat{\bar{\theta}}^{i} \neq 0\right.$ in the limit).

Proposition 16 (Closed-Economy Limit, Permanent Shocks). In the closed-economy limit, in response to a permanent shock $\tilde{s}_{t}^{i}=\tilde{s}_{0}^{i}$, the second-best optimum achieves the first best $\hat{\bar{y}}_{t}^{i}=\hat{\bar{\pi}}_{H, t}^{i}=0$ with

$$
N \hat{\bar{F}} A_{0}^{i}=0 \text { and } \quad \hat{\bar{\theta}}^{i}=\tilde{s}_{0}^{i}
$$

Transfers are particularly useful in the case where shocks are permanent so that $\psi=0$. In this 
case we have $\hat{\bar{\theta}}^{i}=\tilde{s}_{0}^{i}$ and we get perfect stabilization of output and inflation. This striking result illustrates that for rather closed economies in a currency union, even modest transfers can achieve large stabilization benefits. It offers an interesting contrast to the arguments presented by McKinnon (1963) that common currencies are more costly for economies that are more closed, but did not consider transfers. Our result shows that this matters since transfers are more potent in more closed economies.

\subsection{The Role of Fixed Exchange Rates}

We now clarify the role of fixed exchange rates for our results. We assume that only a subset of countries $I \subseteq[0,1]$ are in the currency union. Countries outside the currency union have flexible exchange rates and PCP. ${ }^{31}$

We can write down the corresponding planning problem as follows

$$
\min \frac{1}{2} \int_{0}^{\infty} \int_{0}^{1} e^{-\rho t}\left[\alpha_{\pi}\left(\hat{\bar{\pi}}_{H, t}^{i}\right)^{2}+\left(\hat{\bar{y}}_{t}^{i}\right)^{2}+\alpha_{\theta}\left(\hat{\bar{\theta}}^{i}\right)^{2}+\alpha_{\pi}\left(\pi_{t}^{*}\right)^{2}+\left(\hat{y}_{t}^{*}\right)^{2}\right] d i d t
$$

subject to

$$
\begin{gathered}
\dot{\pi}_{t}^{*}=\rho \pi_{t}^{*}-\kappa_{y} \hat{y}_{t}^{*} \\
\int_{0}^{1} \hat{\bar{\theta}}^{i} d i=0
\end{gathered}
$$

for $i \in I$,

$$
\begin{aligned}
\dot{\hat{\bar{\pi}}}_{H, t}^{i} & =\rho \hat{\bar{\pi}}_{H, t}^{i}-\kappa_{y} \hat{\bar{y}}_{t}^{i}-\lambda \alpha \hat{\bar{\theta}}^{i}, \\
\dot{\bar{y}}_{t}^{i} & =-\hat{\bar{\pi}}_{H, t}^{i}-\dot{\tilde{s}}_{t}^{i} \\
\hat{\bar{y}}_{0}^{i} & =(1-\alpha) \hat{\bar{\theta}}^{i}-\tilde{s}_{0}^{i},
\end{aligned}
$$

and for $i \notin I$,

$$
\dot{\hat{\pi}}_{H, t}^{i}=\rho \hat{\bar{\pi}}_{H, t}^{i}-\kappa_{y} \hat{\bar{y}}_{t}^{i}-\lambda \alpha \hat{\bar{\theta}}^{i}
$$

For countries outside the currency union the only constraint is the Phillips curve. The Euler equation and the initial condition do not appear as constraints because with a flexible exchange rate $\hat{\bar{e}}_{t}^{i}$, they become

$$
\begin{gathered}
\dot{\hat{\bar{y}}}_{t}^{i}=\dot{\hat{\bar{e}}}_{t}^{i}-\hat{\bar{\pi}}_{H, t}^{i}-\dot{\tilde{s}}_{t}^{i}, \\
\hat{\bar{y}}_{0}^{i}=\hat{\bar{e}}_{0}^{i}+(1-\alpha) \hat{\bar{\theta}}^{i}-\tilde{s}_{0}^{i} .
\end{gathered}
$$

Thus, these equations simply define the required value for the path of the exchange rate $\hat{\bar{e}}_{t}^{i}$. As a

\footnotetext{
${ }^{31}$ For countries in a currency union the distinction between LCP and PCP is irrelevant. Here for countries outside a currency union, we have assumed PCP. Under LCP, fluctuations in the exchange rate would not be enough to deliver the first best, and there would remain a role for transfers for countries outside of the currency union, but our analysis of optimal transfers in a currency union would be unchanged.
} 
result, the solution entails $\hat{\bar{\pi}}_{H, t}^{i}=\hat{\bar{y}}_{t}^{i}=\hat{\bar{\theta}}^{i}=0$ for $i \notin I$. These countries do not send or receive transfers. They reach the same allocation as under complete markets and without interventions in financial markets.

Proposition 17 (Countries Outside the Currency Union). For countries outside the currency union, second-best optimal transfers are zero and $\hat{\bar{\theta}}^{i}=0$. They achieve perfect stabilization $\hat{\bar{\pi}}_{H, t}^{i}=\hat{\bar{y}}_{t}^{i}=0$.

This proposition echoes Proposition 10 and shows that in our model, the role of transfers hinges on the fixed exchange rates prevailing in a currency union.

\section{Numerical Illustration}

In Section 5 we set up a framework to analyze optimal transfers in a currency union and provided complete analytical characterizations for two extreme but enlightening special cases: rigid prices and the closed-economy limit. In this section, we undertake a systematic numerical exploration away from these special cases. We also compare the relative effectiveness of transfers and of other macroeconomic stabilization instruments available in currency unions such as domestic fiscal policy and capital controls. We focus throughout on asymmetric shocks, since symmetric shocks can be perfectly stabilized by union-wide monetary policy.

\subsection{Extending and Calibrating the Model}

We start by extending the dynamic model of Sections 4-5. First, we incorporate hand-to-mouth agents. Second, we allow for other instruments such as domestic fiscal policy as well as capital controls. Given space constraints, we only give high-level descriptions of our extensions here. All the details and derivations can be found in a comprehensive and entirely self-contained appendix (Appendix D).

Hand-to-mouth consumers. In the dynamic model of Sections 4-5, agents have access to perfect financial markets and behave like permanent-income consumers. There is ample evidence that this is counterfactual, and that a substantial fraction of consumers act as if they are borrowing constrained or don't have access to financial markets. As we shall see below, accounting for this feature is important to understand the role of transfers.

We therefore enrich the model by introducing a fraction $\chi$ of hand-to-mouth consumers with no access to financial markets who simply consume their income in every period with a high marginal propensity to consume out of transitory income of one. The remaining fraction $1-\chi$ of consumers has perfect access to financial markets and acts in accordance with the permanent-income hypothesis with a low marginal propensity to consume out of transitory income. The baseline model of Sections 4-5 corresponds to the particular case $\chi=0$.

This modeling device strikes an attractive balance between on the one hand capturing consumer heterogeneity and borrowing constraints and on the other hand maintaining enough tractability to 
perform an extensive quantitative normative analysis. A substantial technical difficulty is the derivation of the loss function, which unlike in the baseline model with only permanent-income consumers, requires a second-order approximation of several equilibrium constraints to substitute out the linear terms in the second-order approximation of welfare. This is required to obtain an expression with only second-order terms which can then be maximized subject to a first-order approximation of the equilibrium conditions.

Permanent-income consumers are exactly like in the baseline model. Hand-to-mouth consumers consume their income in every period. This income is composed of their labor income and their share of the profits of domestic firms net of a lump-sum tax (which is negative in the case of a rebate). We assume that profits are distributed uniformly in the population. In every period, international transfers are assumed to flow through the government which receives them and rebates them uniformly across the population.

The optimal policy problem for a given shock then consists in finding the temporal sequence of transfers that minimizes the loss function subject to the log-linearized equilibrium constraints of the economy. The presence of hand-to-mouth consumers requires solving not only for the overall level of transfers, as captured by their net present value as in the case where there are only permanentincome consumers, but also for their timing and hence for the entire path of transfers.

Alternative macroeconomic instruments. In the dynamic model of Sections 4-5, international transfers are the only policy instrument. We extend the model to allow for other policy instruments: capital controls and domestic fiscal policy. In Farhi and Werning (2012), we used the same model presented in Sections 4-5 without hand-to-mouth consumers to study time-varying capital controls as well as time-varying domestic government spending on domestic goods. ${ }^{32,33,34}$ The appendix extends this analysis to allow for hand-to-mouth consumers. While Ricardian equivalence holds in the domestic economy in the model without hand-to-mouth consumers, it does not in the extended model with hand-to-mouth consumers. In the latter case, other dimensions of domestic fiscal policy pertaining to the timing and distribution of domestic taxes become relevant. We consider timevarying government spending financed by uniform taxes on all consumers, time-varying deficits

\footnotetext{
${ }^{32}$ For government spending, our analysis replicates Gali and Monacelli (2008). See Section 4.6 of the NBER working paper version of Farhi and Werning (2012).

${ }^{33}$ It is important to clarify the difference between international transfers and our interpretation of capital controls. Whereas international transfers affect the allocation of consumption across states of the world, capital controls affect the allocation of consumption over time in each state. In our rendition, capital controls are therefore different from the state-contingent portfolio taxes that we analyzed in the complete markets implementation of the static model in Sections 2-3 where this dynamic temporal dimension is entirely absent. The optimal use of capital controls can be derived by studying a planning problem related to (22). The differences are as follows. First, in the objective function (22) and in the New Keynesian Philips curve (23), the constant variable $\hat{\theta}^{i}$ is now replaced by the time-dependent variable $\hat{\theta}_{t}^{i}$. Second, the initial condition (25) now features $\hat{\theta}_{0}^{i}$ instead of $\hat{\bar{\theta}}^{i}$. Third, there is an additional set of constraints $\int e^{-\rho t} \hat{\theta}_{t}^{i} d t=0$ if international transfers are not available but only capital controls are available.

${ }^{34}$ We refer the reader to Appendix B.4 for a treatment of government spending in the context of the static model of Sections 2-3. We characterize the jointly optimal use of international transfers and government spending. Our analysis underscores that both instruments should be used in conjunction. Moreover, we show that our characterization of fiscal unions is robust to the availability of government spending as an additional instrument. We also compare their relative performance depending on a number of deep economic parameters.
} 
in the form of debt-financed tax cuts combined with future tax increases falling uniformly on consumers, time-varying redistribution between consumers, and various combinations of these different options.

Calibration. To allow for maximal comparability between our different instruments, we perform all our numerical simulations within the same extended model with a steady-state share of domestic government spending of 0.2 as in Gali et al. (2007). ${ }^{35}$ We set standard values for $\phi=3$ corresponding to a Frisch elasticity of labor supply of 0.33 , for the elasticity of substitution between varieties $\epsilon=$ 11 , and for the discount rate $\rho=0.06$. In our benchmark calibration we set $\rho_{\delta}=0.79$ ("sticky") corresponding to an average price duration of 5.6 quarters following Altig et al. (2011); we also experiment with $\rho_{\delta}=1.66$ ("more flexible") corresponding to an average price duration of 3 quarters and with fully rigid prices $\rho_{\delta}=0$ ("rigid"). We set the degree of openness to $\alpha=0.4$ ("open") corresponding to a relatively open economy as in Gali and Monacelli (2008); we also experiment with a more closed economy $\alpha=0.1$ ("closed") and with a very closed economy $\alpha=0.01$ ("very closed"). We consider both the version of the model without hand-to-mouth consumers $\chi=0$ ("no HtM"), and with hand-to-mouth consumers. When we consider hand-to-mouth consumers, we follow Gali et al. (2007) and set $\chi=0.5$ ("HtM") which matches the range of estimated values for the proportion of rule-of-thumb consumers documented in Mankiw (2000).

\subsection{Transfers}

In this section, we focus on international transfers. To do so, we assume that all the other instruments are passive or equivalently that they are at their natural level, by which we mean that they are given by their optimal values under flexible prices given the shock. We refer to the corresponding allocation as the natural allocation which is first-best efficient. In our model, the natural level of capital controls is zero as in steady state. By contrast, the natural levels of government spending and taxes are different from their steady state values, depend on the shock, and are not constant over time. However, they do not reflect any macroeconomic stabilization motive and hence represent a passive form of fiscal policy. ${ }^{36,37}$ We start with the case without hand-to-mouth consumers and then

\footnotetext{
${ }^{35}$ Following Gali and Monacelli (2008), we take the utility function to be $(1-v) \frac{C_{t}^{1-\sigma}}{1-\sigma}+v \frac{G_{t}^{1-\sigma}}{1-\sigma}-\frac{N_{t}^{1+\phi}}{1+\phi}$, and we calibrate $v$ to match a steady state government spending share of 0.2 .

${ }^{36}$ That optimal capital controls are zero with flexible prices as in the steady state reflects our focus on coordinated welfare from the perspective of the currency union as opposed to the individual perspective of the country, which neutralizes terms of trade manipulations motives for capital taxation. With flexible prices, optimal government spending and taxes are different from their steady state values, but taxes are uniform across agents and the government budge is balanced in every period.

${ }^{37}$ What constitutes passive fiscal policy is debatable. An alternative to our definition, which we do not pursue, would be to broaden the notion of passive fiscal policy to encompass the "opportunistic" dimension of fiscal policy, which captures the notion that in the presence of nominal rigidities, the microeconomic opportunity cost of fiscal resources might differ from its natural level, and so that on purely microeconomic grounds, there is a case for deviating from the natural fiscal policy. Only the "stimulus" dimension of fiscal policy, which recognizes that with nominal rigidities, there might be a case for fiscal policy to deviate from what would be warranted on purely microeconomic grounds, would then be defined as active. See Werning (2012) for an elaboration of these concepts in the case of government spending. Our
} 
consider the case with hand-to-mouth consumers.

No hand-to-mouth consumers. Figure 2 displays the behavior of the economy with optimal transfers and with no transfers in response to a permanent shock with $\tilde{s}_{t}^{i}=0.05$. The top panel corresponds to $\alpha=0.01$, the middle panel to $\alpha=0.1$ and the bottom panel to $\alpha=0.4$. In this figure, time is measured in years, output is measured in gaps from the natural allocation, and inflation is annualized. The allocation without transfers features deflation and a recession in the short run which vanishes in the long run as prices adjust: the output gap increases from $-5 \%$ to 0 and the inflation rate from $-6 \%$ to 0 . The allocation with transfers features less deflation and smaller recession in the short run, but lower output in the long run. For example, with $\alpha=0.1$, the output gap at impact is only $-1.2 \%$ and the inflation rate $-1.4 \%$. The allocation without transfers is independent of openness $\alpha$. By contrast, the solution with optimal transfers is more stable the more closed the economy (the lower $\alpha$ ). Optimal transfers stabilize the economy more effectively when the economy is more closed.

Figure 3 displays a measure of macroeconomic stabilization due to transfers. As a measure of stabilization, we compute the fraction of the welfare loss without transfers that is eliminated by the introduction of optimal transfers, where welfare losses are computed with respect to the baseline of the natural allocation. We feed in exponentially decaying shocks $\tilde{s}_{t}^{i}=e^{-\psi t} \tilde{s}_{0}^{i}$. We then plot our stabilization measure as a function of openness $\alpha$ and the persistence of the shock as measured by its half life $-\log (0.5) / \psi$. The measure of stabilization is invariant with respect to the size of the shock $\tilde{s}_{0}^{i}$.

Using the same shock, Figure 4 displays $N \hat{\bar{F}} A_{0}^{i}$, which can be interpreted as the net present value of transfers as a fraction of GDP, as a function of the same two parameters. These numbers scale in proportion the size of the shock which we normalize to $\tilde{s}_{0}^{i}=0.05$.

Stabilization is increasing in the persistence of the shock and decreasing in openness. Optimal transfers are increasing in the persistence of the shock starting at zero for fully transitory shocks, but hump-shaped as a function of openness, starting at zero in the closed-economy limit. Significant stabilization is achieved with relatively modest transfers when the economy is relatively closed and shocks are relatively permanent. ${ }^{38}$

Hand-to-mouth consumers. With only permanent-income consumers, transfers are not a well targeted instrument to deal with transitory shocks. For example in the case of a transitory recessive shock, permanent-income consumers spend the permanent value of the transfers in every period,

definition of passive fiscal policy, which identifies as active any deviation from the natural fiscal policy, excludes both the "opportunistic" and the "stimulus" dimensions of fiscal policy. It should be clear that compared to this alternative, our choices only increase the benefits of active fiscal policy.

${ }^{38}$ When prices are perfectly rigid, we saw earlier in Proposition 14 that transfers were zero both when $\alpha=0$ (but stabilization is not zero) and when $\alpha=1$ (and stabilization is zero). When prices are not perfectly rigid, transfers are still exactly zero when $\alpha=0$ but not when $\alpha=1$ (and stabilization is not zero). When the economy is completely open with $\alpha=1$, transfers still influence the domestic wage through a wealth effect and hence inflation as long as prices are not entirely rigid. Therefore, transfers help mitigate the deflation and remain useful for stabilization. 
both in the short run, when their extra spending is needed to stimulate output, and in the long run, when it is not. This changes in the presence of hand-to-mouth consumers with high marginal propensities to consume out of transitory income. The spending of these consumers can be frontloaded by front-loading transfers. As a result, transfers become a more effective instrument to deal with transitory shocks and, in other words, the performance of transfers depends less on the persistence of shocks. A similar intuition applies to the dependence of the performance of transfers on the degree of price rigidity, since greater price flexibility makes the impact of a given shock on macroeconomic stability more transitory given that prices adjust faster. What matters is the persistence of the effects of the shock on macroeconomic stability, either because the shocks themselves are persistent or because price are somewhat sticky. We will make repeated use of this terminology in the discussion below and refer to a shock with transitory effects as a situation where either the shock itself is transitory or where prices are somewhat flexible.

Figure 5 displays the behavior of the economy with optimal transfers and with no transfers in response to a permanent shock with $\tilde{s}_{t}^{i}=0.05$. The top panel corresponds to $\alpha=0.01$, the middle panel to $\alpha=0.1$ and the bottom panel to $\alpha=0.4$. The figure also documents the optimal path of transfers. Because prices are sticky but not perfectly rigid, the effects of the shock on macroeconomic stability are transitory even though the shock is permanent. In the absence of transfers, the economy experiences a recession with a negative output gap and deflation in the short run but the economy equilibrates in the long run returning to zero output gap and no inflation. The equilibrium allocation without transfers is actually identical to that which obtains with no hand-to-mouth consumers. This is due to our Cole-Obstfeld specification: In the absence of transfers and without hand-to-mouth consumers, trade remains balanced and permanent-income consumers end up consuming their income in every period just like hand-to-mouth consumers. This invariance with respect to the fraction of hand-to-mouth consumers breaks down with transfers. As is intuitive, the figure shows that optimal transfers are significantly front-loaded. This contrasts with the baseline case with only permanent-income consumers where the timing of transfers is irrelevant.

Figure 6 displays the amount of stabilization (defined as above) achieved by transfers as a function of openness $\alpha$ and the persistence of the shock as measured by its half life $-\log (0.5) / \psi$. Figure 7 displays the net present value of transfers as a fraction of GDP as a function of the same two parameters. The main differences from the baseline case with only permanent-income consumers $\chi=0$ discussed above are that: Stabilization is much less dependent on the persistence of the shock and the level of transfers is higher. This is because with hand-to-mouth consumers, transfers become a more effective stabilization tool for shocks with transitory effects on the economy. ${ }^{39}$

\footnotetext{
${ }^{39}$ That stabilization increases is intuitive since transfers become more effective. That the net present value of transfers increases is less obvious because in general, making an instrument more effective could either increase or decrease its optimal use. That it could increase its use is intuitive since the instrument becomes more attractive-a form of policy substitution effect. That it could reduce it is due to the fact that a given improvement in the objective can be achieved with a lesser use of the tool-a form of policy income effect. In the case at hand regarding the effectiveness of transfers as the fraction of hand-to-mouth consumers in the economy increases, the policy substitution effect dominates the policy wealth effect, and as a result transfers are used more.
} 


\subsection{Transfers vs. Other Macroeconomic Instruments}

In this section we compare the effectiveness of international transfers to that of other macroeconomic stabilization instruments: capital controls and domestic fiscal policy. Of course international transfers, capital controls, and domestic fiscal policy are different instruments. In general no single instrument can achieve perfect macroeconomic stabilization, and it is best to use all three in conjunction. This is not what interests us here. Instead, we seek to characterize the relative effectiveness of these different instruments. We shall see that their relative ranking varies in intuitive ways depending on the fraction of hand-to-mouth consumers, the openness of the economy, the persistence of shocks, and the degree of nominal rigidity.

\begin{tabular}{|c|c|c|c|c|c|c|c|c|c|c|c|c|c|c|c|}
\hline \multirow[b]{3}{*}{$\begin{array}{l}\text { NOMINAL } \\
\text { RIGIDITIES }\end{array}$} & \multirow[b]{3}{*}{$\begin{array}{l}\text { OPEN- } \\
\text { NESS }\end{array}$} & \multicolumn{14}{|c|}{ PERMANENT } \\
\hline & & \multicolumn{7}{|c|}{ No HtM agents } & \multicolumn{7}{|c|}{ HtM agents } \\
\hline & & No policy & Transfers & $\begin{array}{l}\text { Capital } \\
\text { controls }\end{array}$ & $\begin{array}{c}\text { Gov. } \\
\text { spending }\end{array}$ & $\begin{array}{c}\text { Redistri- } \\
\text { bution }\end{array}$ & Deficits & $\begin{array}{c}\text { Joint fiscal } \\
\text { policy }\end{array}$ & No policy & Transfens & $\begin{array}{l}\text { Capital } \\
\text { controls }\end{array}$ & $\begin{array}{c}\text { Gou } \\
\text { spending }\end{array}$ & $\begin{array}{c}\text { Redistri- } \\
\text { bution }\end{array}$ & Deficits & $\begin{array}{c}\text { Joint fiscal } \\
\text { policy }\end{array}$ \\
\hline \multirow{2}{*}{ More flexible } & Open & $0 \%$ & $25 \%$ & $67 \%$ & $36 \%$ & $0 \%$ & $0 \%$ & $36 \%$ & $0 \%$ & $63 \%$ & $66 \%$ & $36 \%$ & $58 \%$ & $58 \%$ & $62 \%$ \\
\hline & Closed & $0 \%$ & $68 \%$ & $85 \%$ & $36 \%$ & $0 \%$ & $0 \%$ & $36 \%$ & $0 \%$ & $83 \%$ & $85 \%$ & $36 \%$ & $73 \%$ & $73 \%$ & $74 \%$ \\
\hline \multirow{2}{*}{ Sticky } & Open & $0 \%$ & $41 \%$ & $65 \%$ & $36 \%$ & $0 \%$ & $0 \%$ & $36 \%$ & $0 \%$ & $66 \%$ & $64 \%$ & $36 \%$ & $55 \%$ & $55 \%$ & $61 \%$ \\
\hline & Closed & $0 \%$ & $81 \%$ & $82 \%$ & $36 \%$ & $0 \%$ & $0 \%$ & $36 \%$ & $0 \%$ & $87 \%$ & $82 \%$ & $36 \%$ & $71 \%$ & $71 \%$ & $72 \%$ \\
\hline \multirow{2}{*}{ Rigid } & Open & $0 \%$ & $66 \%$ & $0 \%$ & $26 \%$ & $0 \%$ & $0 \%$ & $26 \%$ & $0 \%$ & $66 \%$ & $0 \%$ & $26 \%$ & $0 \%$ & $0 \%$ & $26 \%$ \\
\hline & Closed & $0 \%$ & $94 \%$ & $0 \%$ & $26 \%$ & $0 \%$ & $0 \%$ & $26 \%$ & $0 \%$ & $94 \%$ & $0 \%$ & $26 \%$ & $0 \%$ & $0 \%$ & $26 \%$ \\
\hline
\end{tabular}

\begin{tabular}{|c|c|c|c|c|c|c|c|c|c|c|c|c|c|c|c|}
\hline \multirow[b]{3}{*}{$\begin{array}{l}\text { NOMINAL } \\
\text { RIGIDITIES }\end{array}$} & \multirow[b]{3}{*}{$\begin{array}{c}\text { OPEN- } \\
\text { NESS }\end{array}$} & \multicolumn{14}{|c|}{ TRANSITORY } \\
\hline & & \multicolumn{7}{|c|}{ No HtM agents } & \multicolumn{7}{|c|}{ HtM agents } \\
\hline & & No poligy & Transfers & $\begin{array}{l}\text { Capital } \\
\text { controls }\end{array}$ & $\begin{array}{c}\text { Gou } \\
\text { spending }\end{array}$ & $\begin{array}{c}\text { Redistri- } \\
\text { bution }\end{array}$ & Deficits & $\begin{array}{c}\text { Joint fiscal } \\
\text { policy }\end{array}$ & No policy & Transfers & $\begin{array}{l}\text { Capital } \\
\text { controls }\end{array}$ & $\begin{array}{c}\text { Gou } \\
\text { spending }\end{array}$ & $\begin{array}{c}\text { Redistri- } \\
\text { bution }\end{array}$ & Deficits & $\begin{array}{c}\text { Joint fiscal } \\
\text { policy }\end{array}$ \\
\hline \multirow{2}{*}{ More flexible } & Open & $0 \%$ & $21 \%$ & $83 \%$ & $47 \%$ & $0 \%$ & $0 \%$ & $47 \%$ & $0 \%$ & $76 \%$ & $82 \%$ & $47 \%$ & $73 \%$ & $73 \%$ & $78 \%$ \\
\hline & Closed & $0 \%$ & $57 \%$ & $96 \%$ & $47 \%$ & $0 \%$ & $0 \%$ & $47 \%$ & $0 \%$ & $91 \%$ & $96 \%$ & $47 \%$ & $88 \%$ & $88 \%$ & $89 \%$ \\
\hline \multirow{2}{*}{ Sticky } & Open & $0 \%$ & $29 \%$ & $84 \%$ & $49 \%$ & $0 \%$ & $0 \%$ & $49 \%$ & $0 \%$ & $78 \%$ & $83 \%$ & $49 \%$ & $75 \%$ & $75 \%$ & $80 \%$ \\
\hline & Closed & $0 \%$ & $58 \%$ & $97 \%$ & $49 \%$ & $0 \%$ & $0 \%$ & $49 \%$ & $0 \%$ & $92 \%$ & $97 \%$ & $49 \%$ & $89 \%$ & $89 \%$ & $90 \%$ \\
\hline \multirow{2}{*}{ Rigid } & Open & $0 \%$ & $10 \%$ & $56 \%$ & $26 \%$ & $0 \%$ & $0 \%$ & $26 \%$ & $0 \%$ & $53 \%$ & $54 \%$ & $26 \%$ & $43 \%$ & $43 \%$ & $53 \%$ \\
\hline & Closed & $0 \%$ & $14 \%$ & $79 \%$ & $26 \%$ & $0 \%$ & $0 \%$ & $26 \%$ & $0 \%$ & $81 \%$ & $79 \%$ & $26 \%$ & $67 \%$ & $67 \%$ & $72 \%$ \\
\hline
\end{tabular}

Table 1: Stabilization achieved by different macroeconomic instruments, for permanent shocks (top panel) and transitory shocks with a half life of one year (bottom panel). The measure of the stabilization of a given instrument is the fraction of the welfare loss which is eliminated by allowing for the optimal use of this instrument, where welfare losses are computed with respect to the baseline of the natural allocation. The labels "more flexible", "sticky", and "rigid", correspond to $\rho_{\delta}=1.66$, $\rho_{\delta}=0.79$, and $\rho_{\delta}=0$. The labels "open" and "closed" correspond to $\alpha=0.4$ and $\alpha=0.1$. The labels "No HtM" and "HtM" correspond to $\chi=0$ and $\chi=0.5$.

We compute the amount of stabilization (defined as above) achieved by these different instruments. The results are reported in the top and bottom panels of Table 1. The panels cover not only our benchmark calibration with $\rho_{\delta}=0.79$ ("sticky") and $\alpha=0.4$ ("open"), but also variants with 
$\rho_{\delta}=1.66$ ("more flexible") and $\rho_{\delta}=0$ ("rigid") as well as with $\alpha=0.1$ ("closed"), for an economy without hand-to-mouth consumers ("No HtM") and with hand-to-mouth consumers ("HtM"). We feed in exponentially decaying shocks $\tilde{s}_{t}^{i}=e^{-\psi t} \tilde{s}_{0}^{i}$ with size $\tilde{s}_{0}^{i}$ and half life $-\log (0.5) / \psi$. The top panel focuses on permanent shocks with an infinite half life ("permanent") and the bottom panel on transitory shocks with a half life of one year ("transitory"). We do not need to specify the size of shocks since our stabilization measure is invariant to it.

For each of these cases, we report the stabilization measure obtained for the optimal use of different macroeconomic stabilization instruments. "No policy" corresponds to international transfers at their natural values of zero, capital controls at their natural values of zero, and government spending and taxes at their natural values, i.e. the natural allocation. This constitutes the baseline from which we consider one-at-a-time deviations by allowing for a specific instrument to be optimized at a level different from its natural value. "Transfers" corresponds to time-varying international transfers. "Capital controls" corresponds to time-varying capital controls. "Government spending" corresponds to time-varying deviations of government spending from its natural value financed by contemporaneous and uniform taxes on all consumers. "Redistribution" corresponds to timevarying budget-balanced redistribution between permanent-income consumers and hand-to-mouth consumers. "Deficits" corresponds to time-varying uniform taxes (and government debt) on all consumers. "Joint fiscal policy" corresponds to the combination of all the aforementioned domestic fiscal policy instruments with time-varying government spending and time-varying taxes that are differentiated across consumers.

We start with the case where there are no hand-to-mouth consumers. In this case, Ricardian equivalence holds in the domestic economy and so the timing and distribution of taxes over time are irrelevant. The relative performance of these instruments depends on the parameters of the economy, and in particular on two key parameters: the degree of openness, and the persistence of the effects of the shocks, where as explained above the latter reflects both the persistence of the shock itself and the rigidity of prices. In general, international transfers and capital controls are more potent when the economies are relatively closed. ${ }^{40}$ By contrast, the effectiveness of government spending is independent of the degree of openness - an exact result which relies on our Cole-Obstfeld specification. ${ }^{41}$ International transfers are better suited to deal with shocks with persistent effects whereas capital controls are better suited to deal with transitory shocks. This is because international transfers permanently shift the level of spending whereas capital controls influence the timing of spending. The effectiveness of government spending is less dependent on the persistence of the effects of shocks. ${ }^{42}$

\footnotetext{
${ }^{40}$ For capital controls, we have made this point in formal propositions and simulations in this paper and in Farhi and Werning (2012).

${ }^{41}$ We showed in Farhi and Werning (2012) and we confirm here that under our Cole-Obstfeld specification, optimal government spending, the output gap, and inflation are independent of openness.

${ }^{42}$ The intuition is also supported by the following formal results. In the closed-economy limit, when shocks $\tilde{s}_{t}{ }^{i}=$ $a_{i, t}-a_{t}^{*}$ are permanent, international transfers achieve the first best, while capital controls and government spending do not. In this case, there is no residual macroeconomic stabilization role for capital controls or government spending once international transfers are available. Conversely, in the limit where productivity shocks $\tilde{s}_{t}{ }^{i}=a_{i, t}-a_{t}^{*}$ are purely transitory, optimal international transfers are zero and do not perform any macroeconomic stabilization role, while optimal capital controls and government spending do help stabilize the economy.
} 
We now turn to the case with hand-to-mouth consumers. As explained above, the presence of hand-to-mouth consumers improves the performance of international transfers in dealing with shocks with transitory effects. By contrast, the presence of hand-to-mouth consumers leaves the performance of capital controls and government spending almost unchanged. In fact, in the case of government spending, under our Cole-Obstfeld specification, hand-to-mouth consumers are irrelevant in the sense that their presence does not change the equilibrium allocations, simply because trade remains balanced for any path of government spending, and so permanent-income consumers end up simply consuming their income in every period just like hand-to-mouth consumers. This exact irrelevance does not hold in the case of capital controls, but it remains a good approximation under our various calibrations.

The presence of hand-to-mouth consumers also opens up other dimensions of domestic fiscal policy. For example when facing a recessive shock with transitory effects on the economy, it is optimal to redistribute towards hand-to-mouth consumers in the short run and towards permanent-income consumers in the long run. This is because hand-to-mouth consumers spend the extra revenue when they get it whereas permanent-income consumers spread their loss in revenue by reducing spending uniformly over time. Redistribution towards hand-to-mouth consumers therefore increases spending in the short run when it is needed. Similarly, redistribution towards permanent-income consumers in the long run mitigates the decrease in their spending due to redistribution away from them in the short run. Furthermore, a given amount of transitory redistribution towards hand-tomouth consumers has a more stimulative effect in the short run and a more depressive effect in the long run when the economy is more closed because the corresponding changes in spending fall more on domestic goods. These intuitions explain why the performance of redistribution is higher when the economy is more closed or when the shocks have more transitory effects.

Perhaps surprisingly, optimal deficits achieve exactly the same outcomes as optimal redistribution. This is because under our calibrations, optimal redistribution has the property that the redistribution towards hand-to-mouth consumers in the short run and towards permanent-income consumers in the long run exactly cancel out in a net present value sense, a consequence, we conjecture, of our Cole-Obstfeld specification. As a result, an alternative implementation of the corresponding allocation can be found in the form uniform (across consumers) tax cuts in the short run, financed by debt which is purchased by permanent-income consumers and repaid in the long run by uniform (across consumers) tax increases.

\section{Conclusion}

In this paper, we have characterized fiscal unions as optimal risk sharing arrangements. We have shown that they play a special role within currency unions. We have given a precise description of the effectiveness of such a fiscal union and of the size of the underlying transfers as a function of a small numbers of key characteristics of the currency union, such as the rigidity of prices, the openness of member countries, the asymmetry of shocks, and their persistence. Finally we have 
compared the performance of fiscal unions to that of other macroeconomic stabilization instruments available in currency unions such as capital controls, government spending, fiscal deficits, and redistribution.

\section{References}

Adao, Bernardino, Isabel Correia, and Pedro Teles, "On the relevance of exchange rate regimes for stabilization policy," Journal of Economic Theory, July 2009, 144 (4), 1468-1488.

Altig, David, Lawrence Christiano, Martin Eichenbaum, and Jesper Linde, "Firm-Specific Capital, Nominal Rigidities and the Business Cycle," Review of Economic Dynamics, April 2011, 14 (2), 225247.

Atkeson, Andrew and Tamim Bayoumi, "Private Capital Markets in a Currency Union," in P.R. Masson and M.P. Taylor, eds., Policy Issues in the Operation of Currency Unions, Cambridge University Press, 1993.

Auray, Stephane and Aurelien Eyquem, "On Financial Market Incompleteness, Price Stickiness, and Welfare in a Monetary Union," Annals of Economics and Statistics, 2013, (109-110), 205-233.

Beetsma, Roel M.W.J. and Henrik Jensen, "Monetary and fiscal policy interactions in a microfounded model of a monetary union," Journal of International Economics, December 2005, 67 (2), 320-352.

Benigno, Pierpaolo, "Optimal Monetary Policy for Open Economies," Princeton University Ph.D dissertation 2000.

_ , "Optimal monetary policy in a currency area," Journal of International Economics, July 2004, 63 (2), 293-320.

_ , "Price Stability with Imperfect Financial Integration," Journal of Money, Credit and Banking, 02 2009, 41 (s1), 121-149.

- and Michael Woodford, "Linear-quadratic approximation of optimal policy problems," Journal of Economic Theory, 2012, 147 (1), 1-42.

Clarida, Richard, Jordi Gali, and Mark Gertler, "A Simple Framework for International Monetary Policy Analysis," Journal of Monetary Economics, July 2002, 49 (5), 879-904.

Cole, Harold L. and Maurice Obstfeld, "Commodity Trade and International Risk Sharing: How Much Do Financial Markets Matter?," Journal of Monetary Economics, 1991, 28 (1), 3-24.

Devereux, Michael B. and Charles Engel, "Monetary Policy in the Open Economy Revisited: Price Setting and Exchange-Rate Flexibility," Review of Economic Studies, October 2003, 70 (4), 765-783. 
Farhi, Emmanuel and Ivan Werning, "Dealing with the Trilemma: Optimal Capital Controls with Fixed Exchange Rates," NBER Working Papers 18199, National Bureau of Economic Research, Inc June 2012.

_ and _ , "A Theory of Macroprudential Policies in the Presence of Nominal Rigidities," Econometrica, 09 2016, 84, 1645-1704.

_ and _ , "Fiscal Multipliers: Liquidity Traps and Currency Unions," forthcoming in the Handbook of Macroeconomics, 2017.

_, Gita Gopinath, and Oleg Itskhoki, "Fiscal Devaluations," Review of Economic Studies, 2014,81 (2), 725-760.

Ferrero, Andrea, "Fiscal and monetary rules for a currency union," Journal of International Economics, February 2009, 77 (1), 1-10.

Friedman, Milton, "The Case for Flexible Exchange Rates," in "Essays in Positive Economics," University of Chicago Press, 1953, pp. 157-203.

Gali, Jordi and Tommaso Monacelli, "Monetary Policy and Exchange Rate Volatility in a Small Open Economy," Review of Economic Studies, 07 2005, 72 (3), 707-734.

_ and _, "Optimal monetary and fiscal policy in a currency union," Journal of International Economics, September 2008, 76 (1), 116-132.

_ , J. David Lopez-Salido, and Javier Valles, “Understanding the Effects of Government Spending on Consumption," Journal of the European Economic Association, 03 2007, 5 (1), 227-270.

Kehoe, Patrick and Elena Pastorino, "Financial Markets and Fiscal Unions," NBER Working Papers 23235, National Bureau of Economic Research, Inc January 2017.

Kenen, Peter, "The Theory of Optimum Currency Areas: An Eclectic View," in R.A. Mundell and A.K. Swoboda, eds., Monetary Problems of the International Economy, Chicago University Press, 1969.

Keynes, J.M., “The German transfer problem,” The Economic Journal, 1929, 39 (153), 1-7.

Korinek, Anton and Alp Simsek, "Liquidity Trap and Excessive Leverage," American Economic Review, March 2016, 106 (3), 699-738.

Lucas, Robert E., Jr. and Nancy L. Stokey, "Optimal Fiscal and Monetary Policy in an Economy without Capital," Journal of Monetary Economics, 1983, 12, 55-93.

Mankiw, Gregory N., "The Savers-Spenders Theory of Fiscal Policy," American Economic Review, May 2000, 90 (2), 120-125.

McKinnon, Ronald, “Optimum Currency Areas,” American Economic Review, 1963, 53, 717-724. 
Mundell, Robert, "A Theory of Optimum Currency Areas," American Economic Review, September 1961, 51 (4), 657-665.

_ , "The Economics of Common Currencies," in H.G. Johnson and A.K. Swoboda, eds., Uncommon Arguments for Common Currencies, Allen and Unwin, 1973.

Obstfeld, Maurice and Kenneth Rogoff, "New directions for stochastic open economy models," Journal of International Economics, February 2000, 50 (1), 117-153.

Ohlin, B., "The reparation problem: a discussion," Economic Journal, 1929, 39 (154), 172-182.

Samuelson, Paul, "The Pure Theory of Public Expenditure," The Review of Economics and Statistics, 1954, 36, 387-389.

Schmitt-Grohe, Stephanie and Martin Uribe, "Prudential Policies for Peggers," NBER Working Papers 18031, National Bureau of Economic Research, Inc June 2012.

_ and _ , "Downward Nominal Wage Rigidity, Currency Pegs, and Involuntary Unemployment," Journal of Political Economy, October 2016, 124, 1466-1514.

Werning, Ivan, “Managing a Liquidity Trap: Monetary and Fiscal Policy," 2012. NBER Working Papers. 

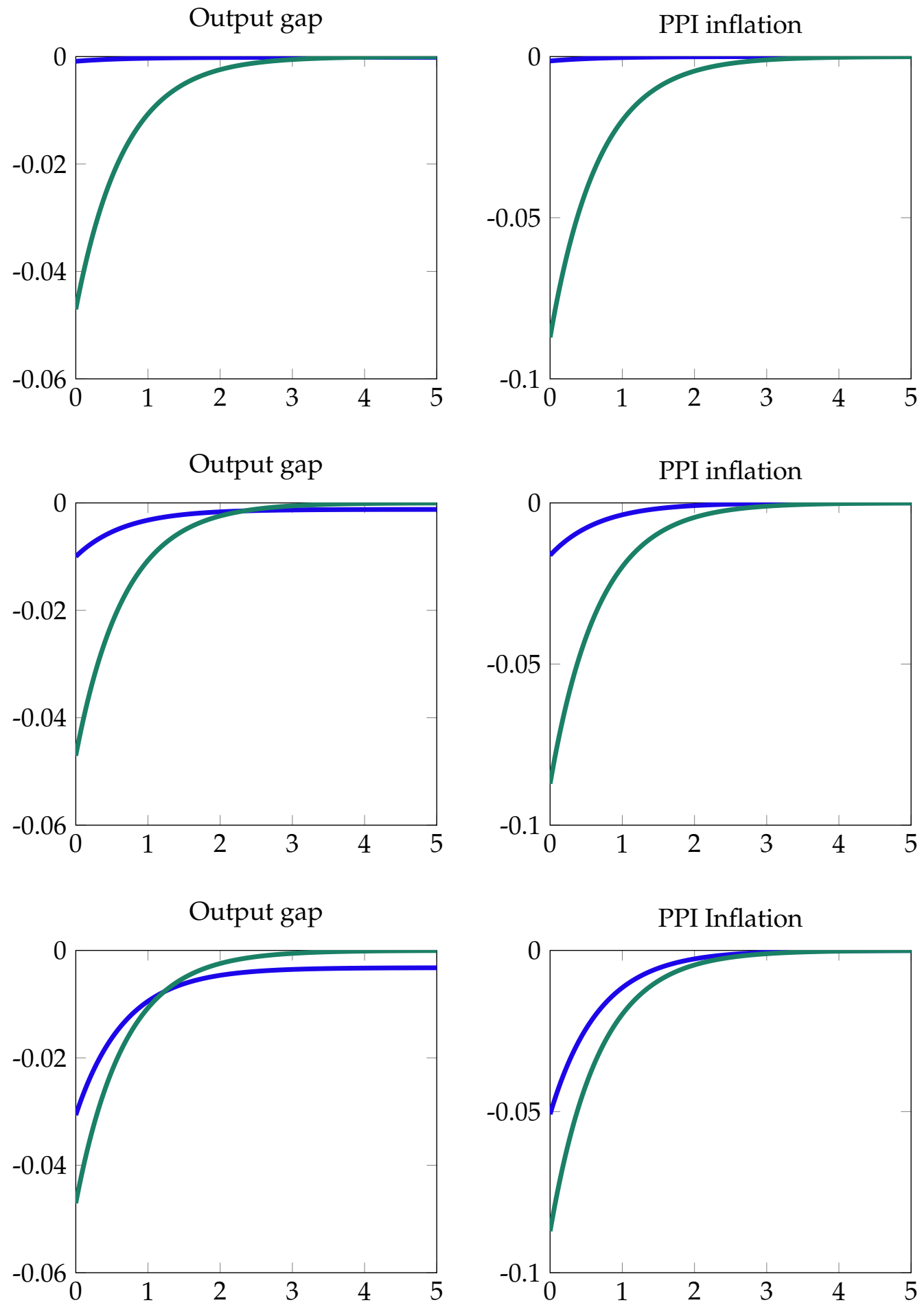

Figure 2: Allocations with optimal transfers (blue) and no transfers (green). The top panel corresponds to $\alpha=0.01$, the middle panel to $\alpha=0.1$ and the bottom panel to $\alpha=0.4$. Inflation is annualized and time is measured in years. Calibration with no hand-to-mouth consumers $\chi=0$. 


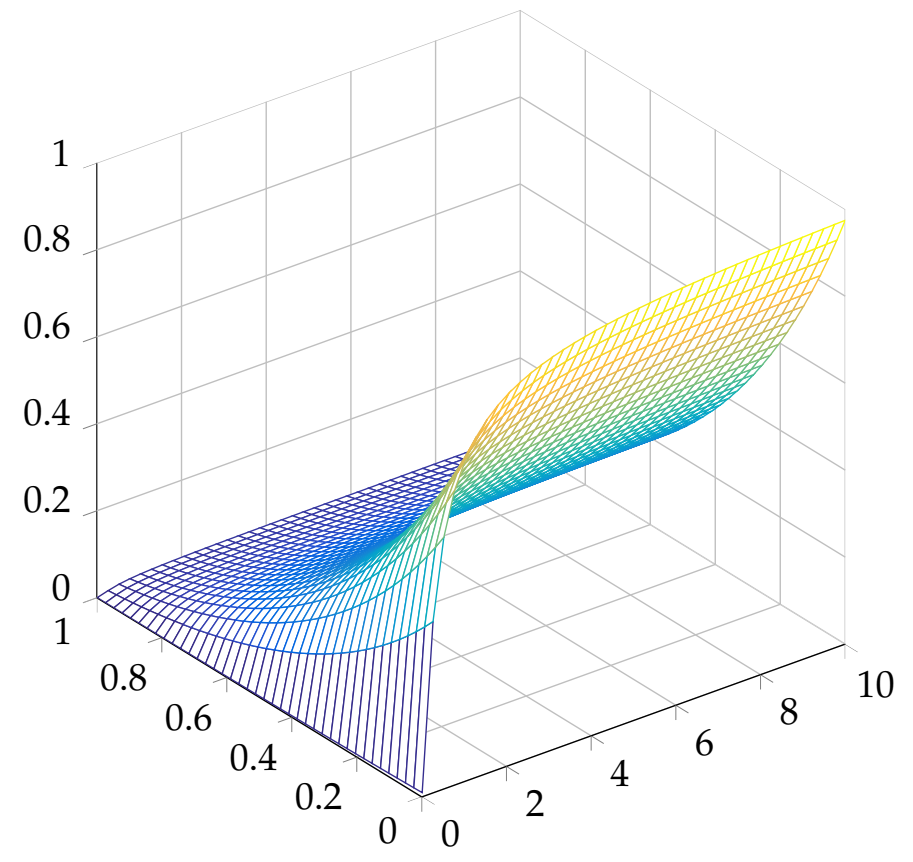

Figure 3: Stabilization effect of transfers as a function of openness measured by $\alpha \in(0,1)$ and persistence measured by the half-life of the shock $-\frac{\log (0.5)}{\psi} \in(0,10)$. The measure of the stabilization of transfers is the fraction of the welfare loss without transfers which is eliminated by the introduction of optimal transfers, where welfare losses are computed with respect to the baseline of the natural allocation. Calibration with no hand-to-mouth consumers $\chi=0$.

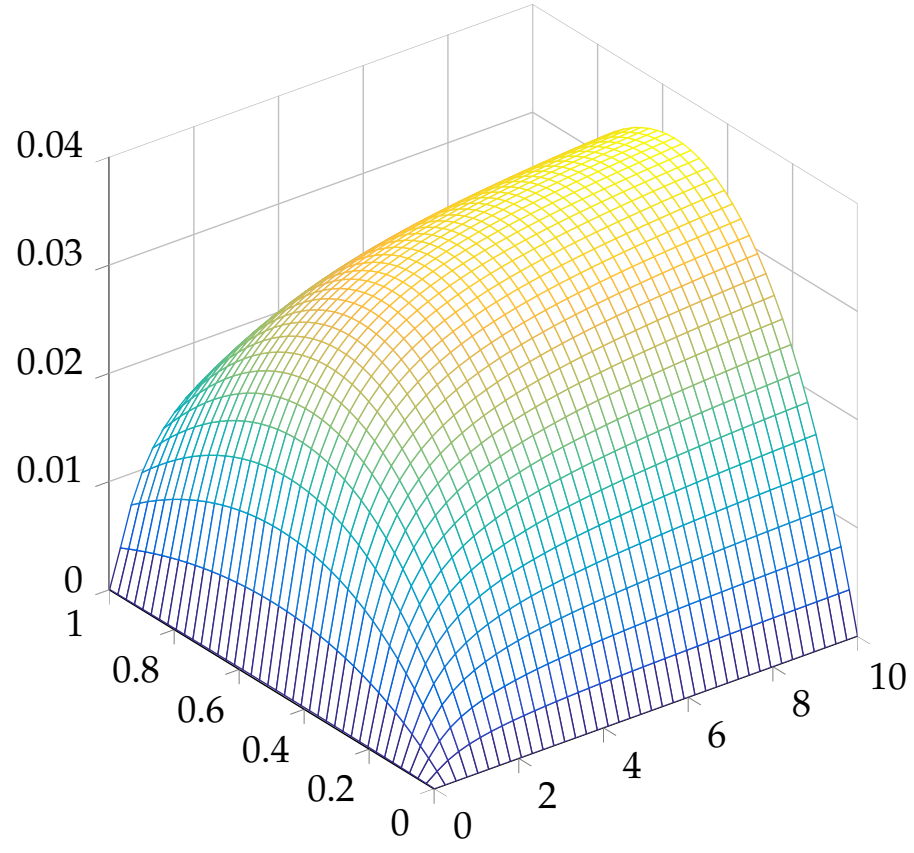

Figure 4: Net present value of transfers as fraction of GDP for a 5\% shock to the terms of trade as a function of openness measured by $\alpha \in(0,1)$ and persistence measured by the half-life of the shock $-\frac{\log (0.5)}{\psi} \in(0,10)$. Calibration with no hand-to-mouth consumers $\chi=0$. 

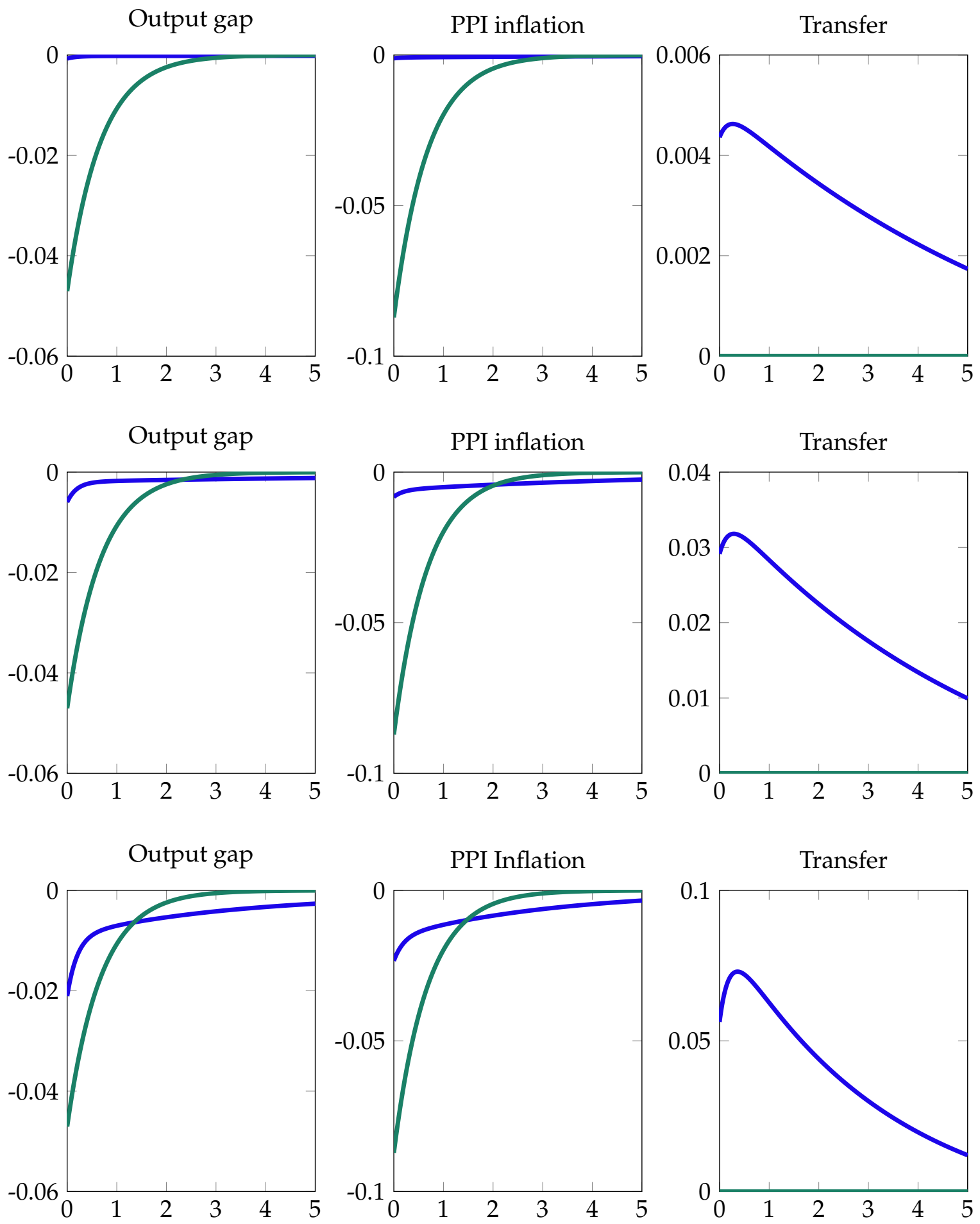

Figure 5: Allocations with optimal transfers (blue) and no transfers (green). The top panel corresponds to $\alpha=0.01$, the middle panel to $\alpha=0.1$ and the bottom panel to $\alpha=0.4$. Inflation is annualized and time is measured in years. Calibration with fraction of hand-to-mouth consumers $\chi=0.5$. 


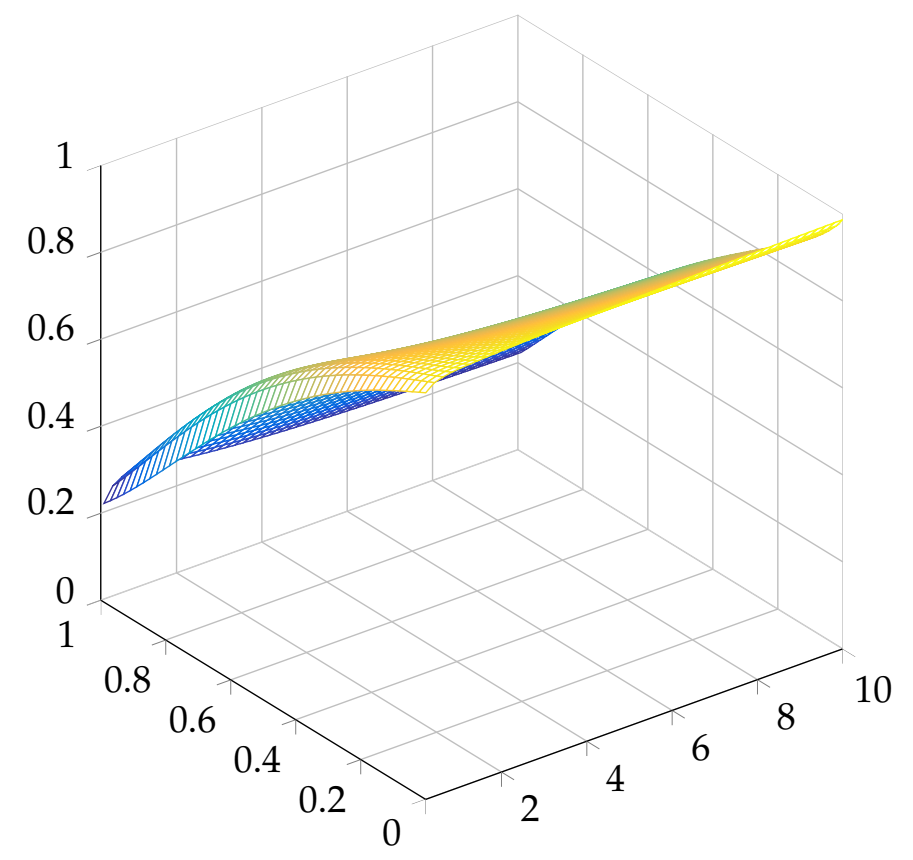

Figure 6: Stabilization effect of transfers as a function of openness measured by $\alpha \in(0,1)$ and persistence measured by the half-life of the shock $-\frac{\log (0.5)}{\psi} \in(0,10)$. The measure of the stabilization of transfers is the fraction of the welfare loss without transfers which is eliminated by the introduction of optimal transfers, where welfare losses are computed with respect to the baseline of the natural allocation. Calibration with fraction of hand-to-mouth consumers $\chi=0.5$.

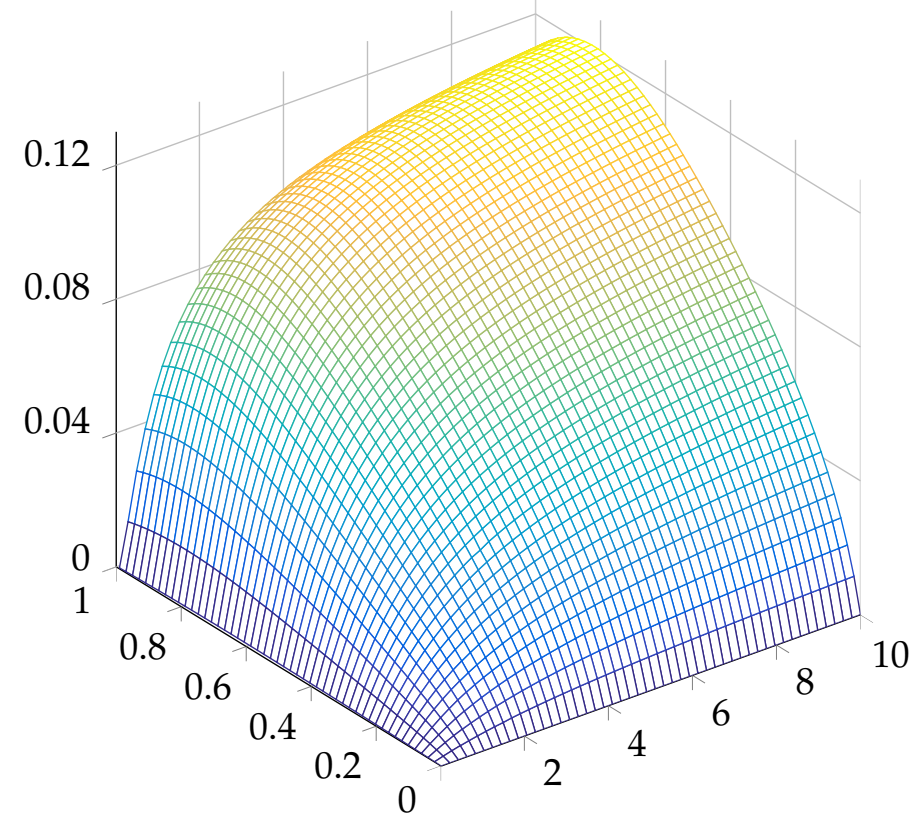

Figure 7: Net present value of optimal transfers as fraction of GDP for a 5\% shock to the terms of trade as a function of openness measured by $\alpha \in(0,1)$ and persistence measured by the half-life of the shock $-\frac{\log (0.5)}{\psi} \in(0,10)$. Calibration with fraction of hand-to-mouth consumers $\chi=0.5$. 


\section{A Online Appendix: Proofs and Details for Static Model in Sec- tions 2-3}

\section{A.1 Proof of Proposition 1}

We have already proved that the conditions in the proposition are necessary for an allocation together with prices to form part of an equilibrium with complete markets. We now need to establish these conditions are sufficient. The proof is constructive. Start with an allocation together with prices that satisfy these conditions. We choose wages $W^{i}(s)$ to satisfy the labor-leisure condition (5) for each $i \in I$ and $s \in S$. Given some set of state prices $Q(s)$, we pick portfolio taxes $\tau_{D}^{i}(s)$ to satisfy the risk sharing condition (3) for each $i \in I$ and $s \in S$. Note a first dimension of indeterminacy here: we can always multiply state prices $Q(s)$ and portfolio taxes $1+\tau_{D}^{i}(s)$ by some arbitrary common function $\lambda(s)$ of $s$. We then pick labor taxes $\tau_{L}^{i}$ to satisfy the price setting equation (6). Finally, for a given set of ex-post fiscal transfers $\hat{T}^{i}(s)$ that satisfy the country budget constraint $\int Q(s) \hat{T}^{i}(s) \pi(s) d s=\int Q(s)\left[P_{T}(s)\left(C_{T}^{i}(s)-E_{T}^{i}(s)\right)\right] \pi(s)$ and the condition that aggregate net international transfers are zero in every state (8), we compute transfers to households $T^{i}(s)$ using the government budget constraint (7). We can then compute the required portfolio positions $D^{i}(s)$ using the ex-post household budget constraint (2). These choices guarantee that the ex-ante household budget constraint (1) is verified. Note a second dimension of indeterminacy, as we have some degree of freedom in choosing ex-post fiscal transfers $\hat{T}^{i}(s)$.

\section{A.2 Proof of Proposition 2}

We have already proved that the conditions in the proposition are necessary for an allocation together with prices to form part of an equilibrium with complete markets. We now need to establish these conditions are sufficient. The proof is constructive. Start with an allocation together with prices that satisfy these conditions. We choose wages $W^{i}(s)$ to satisfy the labor-leisure condition (5) for each $i \in I$ and $s \in S$. We then pick labor taxes $\tau_{L}^{i}$ to satisfy the prices setting equation (12). We choose transfers $T^{i}(s)$ to satisfy the household budget constraint (11). We then choose ex-post fiscal transfers $\hat{T}^{i}(s)$ to satisfy the government budget constraint (13). We can verify that these choices satisfy (8).

\section{A.3 Price Setting with Constant Elasticity of Substitution}

We have

$$
1-\frac{\int \tau^{i}(s) U_{C_{N T}}^{i}(s) C_{N T}^{i}(s) \pi(s) d s}{\int U_{C_{N T}}^{i}(s) C_{N T}^{i}(s) \pi(s) d s}=\frac{1}{1+\tau_{L}^{i}} \frac{\varepsilon-1}{\varepsilon} .
$$


We can rewrite the first-order condition for $P_{N T}^{i}$ as

$$
\int \frac{\alpha_{p}^{i}(s)}{\alpha^{i}(s)} p^{i}(s) \alpha^{i}(s) C_{T}^{i}(s) \frac{1}{p^{i}(s)} U_{C_{T}}^{i}(s) \tau^{i}(s) \pi(s) d s=0
$$

If $\frac{\alpha_{p}^{i}(s)}{\alpha^{i}(s)} p^{i}(s)$ is constant then this implies that

$$
\int C_{N T}^{i}(s) U_{C_{N T}}^{i}(s) \tau^{i}(s) \pi(s) d s=0
$$

Thus in this case $\frac{1}{1+\tau_{L}^{i}} \frac{\varepsilon-1}{\varepsilon}=1$ or $\tau_{L}^{i}=-1 / \varepsilon$.

\section{A.4 Proof of Proposition 7}

Consider an equilibrium such that $\tau^{i}(s) \neq 0$ for some $i \in I, s \in S$. Assume, towards a contradiction, that the allocation is constrained Pareto efficient.

We consider two cases in turn. First, suppose that $V_{C_{T}}^{i}(s)=U_{C_{T}}^{i}(s)\left(1+\frac{\alpha^{i}(s)}{p^{i}(s)} \tau^{i}(s)\right)<0$ for some set $\Omega \subset I \times S$ of positive measure of countries and states. Define the sections $\Omega(s)=\{i:(i, s) \in \Omega\}$. Then there exists a perturbation that for each $s \in S:$ (a) lowers $C_{T}^{i}(s)$ for $i \in \Omega(s)$ and improves welfare $V^{i}(s)$; (b) increases $C_{T}^{i}(s)$ for $i \notin \Omega(s)$ and improves welfare $V^{i}(s)$; and (c) satisfies the resource constraint $\int C_{T}^{i}(s) d i=\int E_{T}^{i}(s) d i$. This perturbation is feasible and creates a Pareto improvement, a contradiction.

Next, consider the case where $1+\frac{\alpha^{i}(s)}{p^{i}(s)} \tau^{i}(s) \geq 0$ for all $i \in I, s \in S$. For each state $s$ consider ranking countries by their weighted labor wedge $\frac{\alpha^{i}(s)}{p^{i}(s)} \tau^{i}(s)$. By Proposition 6 it must be that

$$
\frac{1+\frac{\alpha^{i}(s)}{p^{i}(s)} \tau^{i}(s)}{1+\frac{\alpha^{i^{\prime}}(s)}{p^{i^{\prime}}(s)} \tau^{i^{\prime}}(s)}=\frac{1+\frac{\alpha^{i}(s)}{p^{i}(s)} \tau^{i}\left(s^{\prime}\right)}{1+\frac{\alpha^{i^{\prime}}(s)}{p^{i^{\prime}}(s)} \tau^{i^{\prime}}\left(s^{\prime}\right)}
$$

for all $i, i^{\prime}, s$ and $s^{\prime}$. This implies that the ranking must be the same in all states $s$. It follows that there is a country $i^{*}$ that is at top of the ranking for all states $s$, i.e. $i^{*} \in \cap_{s \in S} \arg \max _{i \in I} \frac{\alpha^{i}(s)}{p^{i}(s)} \tau^{i}(s)$. Proposition 5 then implies that this country has a positive labor wedge: $\tau^{i^{*}}(s) \geq 0$ for all s. Proposition 4 then implies that $\tau^{i^{*}}(s)=0$ for all $s$. Therefore we have that $\tau^{i}(s) \leq 0$ for all $i \in I, s \in S$. Proposition 5 then implies that actually $\tau^{i}(s)=0$ for all $i \in I, s \in S$. 


\section{B Online Appendix: Extensions for Static Model in Sections 2-3}

\section{B.1 Sticky Wages}

In order to have a well defined wage setting problem we assume that labor services are produced by combining a variety of differentiated labor inputs according to the constant returns CES technology

$$
N^{i}(s)=\left(\int_{0}^{1} N^{i, h}(s)^{1-\frac{1}{\varepsilon_{w}}} d h\right)^{\frac{1}{1-\frac{1}{\varepsilon_{w}}}} .
$$

The rest of the technology is as before. We assume that in each country there is a continuum of workers $h \in[0,1]$, each supplying a particular variety $h \in[0,1]$ with preferences

$$
\int U^{i}\left(C_{N T}^{i, h}(s), C_{T}^{i, h}(s), N^{i, h}(s) ; s\right) \pi(s) d s .
$$

The budget constraints are the same as before

$$
\begin{gathered}
\int D^{i, h}(s) Q(s) \pi(s) d s \leq 0, \\
P_{N T}^{i}(s) C_{N T}^{i, h}(s)+P_{T}(s) C_{T}^{i, h}(s) \leq\left(1-\tau_{L}^{i}\right) W^{i, h} N^{i, h}(s) \\
+P_{T}(s) E_{T}^{i}(s)+\Pi^{i}(s)+T^{i}(s)+\left(1+\tau_{D}^{i}(s)\right) D^{i, h}(s),
\end{gathered}
$$

except that the wage $W^{i, h}$ is now specific to each worker $h$ but independent of $s$ because wages are set in advance of the realization of the state $s$. Note that prices of non-traded goods are now state-contingent. For convenience, we now assume that the worker pays for the labor tax; firms are untaxed.

Workers set their own wages $W^{i, h}$ taking into account that in each state of the world $s$ labor demand is given by $N^{i}(s)\left(W^{i, h} / W^{i}\right)^{-\varepsilon_{w}}$ where $W^{i}=\left(\int\left(W^{i, h}\right)^{1-\varepsilon_{w}} d h\right)^{1 /\left(1-\varepsilon_{w}\right)}$ is the wage index for labor services. In a symmetric equilibrium, all workers set the same wage $W^{i, h}=W^{i}$, and consume and work the same so that $C_{N T}^{i, h}(s)=C_{N T}^{i}(s), C_{T}^{i, h}(s)=C_{T}^{i}(s)$ and $N^{i, h}(s)=N^{i}(s)$. The wage $W^{i}$ is given by

$$
W^{i}=\frac{1}{1-\tau_{L}^{i}} \frac{\epsilon_{w}}{\epsilon_{w}-1} \frac{\int-N^{i}(s) U_{N}^{i}(s) \pi(s) d s}{\int \frac{U_{C_{N T}}^{i}(s)}{P_{N T}^{i}(s)} N^{i}(s) \pi(s) d s} .
$$

All varieties sell at the same price so that $P_{N T}^{i, j}(s)=P_{N T}^{i}(s)$. This price is given by

$$
P_{N T}^{i}(s)=\frac{\epsilon}{\epsilon-1} \frac{W^{i}}{A^{i}(s)} .
$$

All the results that we derived in the version of the model with sticky prices carry through with no modification to this specification with sticky wages. In particular, Propositions 1-12 are still 
valid. However, the corresponding allocations can be different than under sticky prices if there are productivity shocks.

\section{B.2 Limited Commitment}

Explicit or implicit insurance (risk sharing) arrangements inevitably raise concerns of incentives. We have abstracted from these considerations, not because we believe them to be unimportant, but in order to isolate the effects that our aggregate demand externality has on optimal risk sharing. Modeling limits to insurance due to incentive problems requires making specific choices about the underlying shocks, the asymmetry of information, the available monitoring technologies, or the type of commitment problem, etc. Although the possibilities are vast and exploring them all is beyond the scope of this paper, we believe the main insights of our analysis would carry over. ${ }^{43}$

In the online appendix B.3, we analyze an example with moral hazard. Here instead, we develop an example with limited commitment. Consider the implementation with incomplete markets and international transfers, where all international risk sharing occurs through international transfers. Ex post, in every state of the world $s$, some countries $i$ are net contributors to the union with $\hat{T}^{i}(s) \leq 0$, and some countries $i$ are net beneficiaries with $\hat{T}^{i}(s) \geq 0$. This poses no particular problem to the extent that there exists a strong enough union-wide institutional enforcement mechanism. But with imperfect enforcement and limited commitment, the concern arises that governments of ex-post net contributor countries do no in fact contribute the transfers that were agreed upon ex ante behind the veil of ignorance before the realization of the shock.

To make things stark, consider the extreme case where there is no institutional enforcement mechanism. Governments can default on their promised transfers $\hat{T}^{i}(s)$, and have no ability to commit. We assume that default leads to a utility loss for which we adopt a flexible parametrization $K^{i}(s)$. The planning problem can now be written as

$$
\max _{P_{T}, P_{N T}^{i}, C_{T}^{i}(s)} \iint \lambda^{i} V^{i}\left(C_{T}^{i}(s), \frac{P_{T}}{P_{N T}^{i} ; s}\right) \pi(s) d i d s
$$

subject to

$$
\int C_{T}^{i}(s) d i=\int E_{T}^{i}(s) d i
$$

and

$$
V^{i}\left(C_{T}^{i}(s), \frac{P_{T}}{P_{N T}^{i}} ; s\right) \geq V^{i}\left(E_{T}^{i}(s), \frac{P_{T}}{P_{N T}^{i}} ; s\right)-K^{i}(s) .
$$

The only difference introduced by the limited commitment problem is the presence of the incen-

\footnotetext{
${ }^{43}$ In practice of course, institutional mechanisms exist to mitigate these agency problems. For example, most fiscal unions such as the US channel a large part of their transfers through more or less ex-ante-rules-based automatic stabilizers (through the unemployment insurance program, federal income and social security taxes, bailout funds), probably for reasons of political acceptability and transparency, but also to mitigate the difficulties associated with collective and distributing discretionary ex-post transfers in a world with limited commitment. Another example is state debt-limit in the US, or collective budget procedures and enforcement mechanisms that already exist in Europe.
} 
tive compatibility constraint (30), requiring each country to be better off sticking to the fiscal union arrangement than defaulting and reverting to autarky while experiencing the endowment loss associated with default.

Let $\mu>0$ be the multiplier on (29) and $v^{i}(s) \geq 0$ be the multiplier on (30). The condition for constrained efficient risk sharing becomes

$$
U_{C_{T}}^{i}(s)\left[1+\frac{\alpha^{i}(s)}{p^{i}(s)} \tau^{i}(s)\right]\left[1+v^{i}(s)\right]=\mu .
$$

By contrast, the corresponding corresponding condition for a country outside the currency union is

$$
U_{C_{T}}^{i}(s)\left[1+v^{i}(s)\right]=\mu .
$$

Condition (32) shows that even with flexible exchange rates, limited commitment endogenously limits insurance (risk sharing) possibilities. A high value of the multiplier $v^{i}(s)$ indicates that it is relatively tempting for country $i$ to default in a state $s$. The optimal contract then adjusts the transfer $\hat{T}^{i}(s)$ and the traded goods consumption $C_{T}^{i}(s)$ so that default is prevented. Condition (31) shows how the optimal provision of insurance (risk sharing) and incentives must be modified when the country is in a currency union. The provision of incentives requires the private consumption of traded goods to vary with the realization of government consumption. Because prices are sticky, this generates a non-zero pattern of labor wedges $\tau^{i}(s)$. This in turn opens up a wedge between the social and private marginal utility of income, which creates another force agains the perfect equalization of consumption of traded goods across states for each country.

This example shows that the optimal risk sharing arrangements are different for countries that belong to a currency union than that for countries who have a flexible exchange rate. This is true with or without enforcement frictions. In both cases, the optimal arrangement involves a key sufficient statistic, the social marginal utility of transfers given by $U_{C_{T}}^{i}(s)\left[1+\frac{\alpha^{i}(s)}{p^{i}(s)} \tau^{i}(s)\right]$ for a country in a currency union and given by $U_{C_{T}}^{i}(s)$ for a country outside a currency union.

\section{B.3 Moral Hazard}

Suppose that the government can exert effort $e$ ex ante to affect the distribution of the endowment of the traded good ex post, but that effort $e$ is not observable, creating a moral hazard problem. We focus on a single country $i \in[0,1]$. We assume that the shock $s$ is purely idiosyncratic and only affects the value of the endowment $E_{T}^{i}(s)$ in country $i$. Naturally, monetary policy at the union level should not react to to the idiosyncratic shocks $s$ of an infinitesimal country, so that $P_{T}(s)=P_{T}$ is constant. These assumptions simplify the exposition. The principal-agent problem is then

$$
\max _{P_{N T}^{i}, C_{T}^{i}(s), e} \int V^{i}\left(C_{T}^{i}(s), \frac{P_{T}}{P_{N T}^{i}}\right) \pi(s \mid e) d s-h(e)
$$


subject to

$$
\int\left(C_{T}^{i}(s)-E_{T}^{i}(s)\right) \pi(s \mid e) d s \leq 0
$$

and

$$
\int V^{i}\left(C_{T}^{i}(s), \frac{P_{T}}{P_{N T}^{i}}\right) \pi(s \mid e) d s-h(e) \geq \int V^{i}\left(C_{T}^{i}(s), \frac{P_{T}}{P_{N T}^{i}}\right) \pi\left(s \mid e^{\prime}\right) d s-h\left(e^{\prime}\right) \quad \text { for all } e^{\prime} .
$$

The first constraint (34) simply conditions the average level of expected transfers; this reflects the fact that insurance is priced fairly i.e. $(Q(s)=1)$, since the shock is experienced by a single country and does not affect aggregate resources at the union level. The last constraint (35) is the incentive compatibility condition, requiring the country's effort to be optimal, taking the schedule $C_{T}^{i}(s)$ as given.

In the absence of nominal rigidities or for a country with flexible exchange rates and independent monetary policy, we would solve the same problem but using $V^{i *}\left(C_{T}^{i}(s)\right)=\max _{p^{i}} V^{i}\left(C_{T}^{i}(s), p^{i}\right)$ in place of $V^{i}\left(C_{T}^{i}(s), \frac{P_{T}}{P_{N T}^{i}}\right)$. Note that $V^{i}\left(C_{T^{\prime}}^{i} p^{i}\right) \leq V^{i *}\left(C_{T}^{i}\right)$ with equality at a single value of $C_{T}^{i}$, so that $V^{i *}$ is an upper envelope of $V^{i}$. When prices are rigid it is as if the country were more risk averse, in the sense described earlier. In the presence of moral hazard, higher risk aversion affects the optimal insurance (risk sharing) contract $C_{T}^{i}(\cdot)$.

Consider the planning problem (33). Let $\mu$ be the multiplier on (34) and $d v\left(e^{\prime}\right)$ be the measure multiplier on (35). The corresponding for constrained efficient risk sharing becomes

$$
U_{C_{T}}^{i}(s)\left[1+\frac{\alpha^{i}(s)}{p^{i}(s)} \tau^{i}(s)\right]\left[1+\int \frac{\pi(s \mid e)-\pi\left(s \mid e^{\prime}\right)}{\pi(s \mid e)} d v\left(e^{\prime}\right)\right]=\mu .
$$

By contrast, the corresponding corresponding condition for a country outside the currency union is

$$
U_{C_{T}}^{i}(s)\left[1+\int \frac{\pi(s \mid e)-\pi\left(s \mid e^{\prime}\right)}{\pi(s \mid e)} d v\left(e^{\prime}\right)\right]=\mu .
$$

Condition (37) shows that even with flexible exchange rates, moral hazard endogenously limits insurance (risk sharing) possibilities. There is a meaningful tradeoff between insurance (risk sharing) and incentives and providing incentives for the country's government to exert the adequate level effort requires the government to have "skin in the game". The private consumption of traded goods must vary with the realization of government spending on traded goods. It must be high whenever the particular realization of government spending is more likely (as measured by the likelihood ration $\left.\frac{\pi(s \mid e)-\pi\left(s \mid e^{\prime}\right)}{\pi(s \mid e)}\right)$ under the desired effort level than under alternative effort levels that the government is tempted to exert (as measured by the measure multiplier $d v\left(e^{\prime}\right)$ on the corresponding incentive compatibility constraint). This is accomplished by reducing the level of transfers to the country when government spending on traded goods is high.

Condition (36) shows how the optimal provision of insurance (risk sharing) and incentives must be modified when the country is in a currency union. The provision of incentives requires the pri- 
vate consumption of traded goods to vary with the realization of government consumption. Because prices are sticky, this generates a non-zero pattern of labor wedges $\tau^{i}(s)$. This in turn opens up a wedge between the social and private marginal utility of income, which creates another force agains the perfect equalization of consumption of traded goods across states. As a result, the optimal insurance insurance (risk sharing) arrangements are different for countries that belong to a currency union than that for countries who have a flexible exchange rate.

\section{B.4 Government Spending}

We introduce government spending in the model. We characterize the joint optimal use of international transfers and government spending. Our analysis underscores that both instruments should be used in conjunction. Moreover, we show that our characterization of fiscal unions is robust to the availability of government spending as an additional instrument. We also compare their relative performance depending on a number of deep economic parameters by studying a few limit cases.

Introducing government spending. Following the literature, we focus on the case where government spending is concentrated on non-traded goods, which we view as the most practically relevant case. ${ }^{44}$ In each state $s$ and country $i$, the government spends $P_{N T}^{i} G_{N T}^{i}(s)$ to finance government consumption of $G_{N T}^{i}(s)$ of non-traded goods. As is standard, we capture agents' preferences of government consumption by including it in the utility function and write

$$
U^{i}\left(G_{N T}^{i}(s), C_{N T}^{i}(s), C_{T}^{i}(s), N^{i}(s) ; s\right)
$$

for the state-s utility function of country $i$ agents. We assume that that preferences are weakly separable over government consumption on the one hand, and private consumption and labor on the other hand. In addition, we continue to assume that preferences over consumption goods are weakly separable from labor, and that the preference over consumption goods are homothetic.

Apart from that, there are only minor differences with the setup of the main model. These differences involve the government budget constraint, the resource constraint for non-traded goods, and the price setting conditions. ${ }^{45}$ Our implementability results in Propositions 1 and 2 can be extended in a straightforward way.

\footnotetext{
${ }^{44}$ For example Beetsma and Jensen (2005) and Gali and Monacelli (2008) introduce government spending on domestic goods in models where all goods are traded, with or without home bias in consumption. The natural equivalent in our setup is to study government spending on non-traded goods. We have also analyzed government spending on traded goods. The analysis is available upon request.

${ }^{45}$ The government budget constraint is now $T^{i}(s)+P_{N T}^{i} G_{N T}^{i}(s)=\tau_{L}^{i} W^{i}(s) N^{i}(s)-\tau_{D}^{i}(s) D^{i}(s)+\hat{T}^{i}(s)$. The resource constraint for non-traded goods is now $C_{N T}^{i}(s)+G_{N T}^{i}(s)=A^{i}(s) N^{i}(s)$. The price setting constraint is now $P_{N T}^{i}=$ $\left(1+\tau_{L}^{i}\right) \frac{\varepsilon}{\varepsilon-1} \frac{\int \frac{Q(s)}{1+\tau_{D}^{i}(s)} \frac{W^{i}(s)}{A^{i}(s)}\left[C_{N T}^{i}(s)+G_{N T}^{i}(s)\right] \pi(s) d s}{\int \frac{Q(s)}{1+\tau_{D}^{i}(s)}\left[C_{N T}^{i}(s)+G_{N T}^{i}(s)\right] \pi(s) d s}$.
} 
Second-Best Planning problem. In order to write down the second-best Ramsey planning problem jointly characterizing international transfers and government spending, we modify the indirect utility function. We define

$$
\tilde{V}^{i}\left(G_{N T}^{i}(s), C_{T}, p ; s\right)=U^{i}\left(G_{N T}^{i}(s), \alpha^{i}(p ; s) C_{T}, C_{T}, \frac{\alpha^{i}(p ; s) C_{T}+G_{N T}^{i}(s)}{A^{i}(s)} ; s\right) .
$$

In an equilibrium with $G_{N T}^{i}(s), C_{T}^{i}(s)$ and $p^{i}(s)$, ex post welfare in state $s$ in country $i$ is then given by

$$
\tilde{V}^{i}\left(G_{N T}^{i}(s), C_{T}^{i}(s), p^{i}(s) ; s\right) .
$$

The second-best planning problem is

$$
\max _{G_{N T}^{i}(s), P_{T}(s), P_{N T}^{i}, C_{T}^{i}(s)} \iint \tilde{V}^{i}\left(G_{N T}^{i}(s), C_{T}^{i}(s), \frac{P_{T}(s)}{P_{N T}^{i}} ; s\right) \lambda^{i} \pi(s) d i d s
$$

subject to

$$
\int C_{T}^{i}(s) d i=\int E_{T}^{i}(s) d i .
$$

We can solve this planning problem recursively by defining

$$
V^{i}\left(C_{T}, p ; s\right)=\max _{G_{N T}^{i}(s)} U^{i}\left(G_{N T}^{i}(s), \alpha^{i}(p ; s) C_{T}, C_{T}, \frac{\alpha^{i}(p ; s) C_{T}+G_{N T}^{i}(s)}{A^{i}(s)} ; s\right),
$$

and then solving

$$
\max _{P_{T}(s), P_{N T}^{i}, C_{T}^{i}(s)} \iint V^{i}\left(C_{T}^{i}(s), \frac{P_{T}(s)}{P_{N T}^{i}} ; s\right) \lambda^{i} \pi(s) d i d s
$$

subject to

$$
\int C_{T}^{i}(s) d i=\int E_{T}^{i}(s) d i
$$

Constrained Pareto efficient allocations. With these notations, the analysis is identical to that of the model without government spending. Indeed, the derivatives of the indirect utility function $V^{i}\left(C_{T}, p ; s\right)$ are given by exactly the same formula as in Proposition 3 , and as a result, Propositions 4-10 as well as Proposition 12 carry through without any modification. ${ }^{46}$ Hence our analysis of fiscal unions is robust to the availability of government spending as an additional instrument.

Of course, this does not mean that the resulting allocation is unchanged. Away from this case, optimal government spending can reduce the deviations of the labor wedge $\tau^{i}(s)$ from zero, but it does not eliminate them. ${ }^{47}$ There are two informative ways to write the optimality condition for government spending, both of which follow directly from the definition of $V^{i}\left(C_{T}, p ; s\right)$ in equation

\footnotetext{
${ }^{46}$ The exact conditions in Proposition 11 for the constrained efficiency of the complete markets equilibrium without portfolio taxes are different in the presence of government spending.

${ }^{47}$ Formally, this is true except in the knife-edge cases where the optimal allocation with flexible prices can be implemented with a fixed exchange rate.
} 
(38):

$$
U_{G_{N T}}^{i}(s)=-\frac{1}{A^{i}(s)} U_{N}^{i}(s)
$$

or

$$
U_{G_{N T}}^{i}(s)=\left(1-\tau^{i}(s)\right) U_{C_{N T}}^{i}(s) .
$$

To understand these formulas, it is best to analyze first the case when prices or exchange rates are flexible. Optimal government spending is then characterized by equation (39) or equation (40) with $\tau^{i}(s)=0$. Both equations equalize the marginal benefit $U_{G_{N T}}^{i}(s)$ of government consumption with its marginal cost, but express the marginal cost in two different (but equivalent) ways. Equation (39) expresses the marginal cost $-\frac{1}{A^{i}(s)} U_{N}^{i}(s)$ in terms of the marginal increase labor that would be required to service the marginal increase in government consumption, while equation (40) with $\tau^{i}(s)=0$ expresses the marginal cost $U_{C_{N T}}^{i}(s)$ in terms of the marginal reduction in private consumption that would be required to service the marginal increase in government consumption. These are two equivalent ways of stating the Samuelson rule (see Samuelson 1954) for the optimal provision of public goods.

Depending on which of these formulations one prefers to focus on, rigid prices and fixed exchange rates either require no deviation from the Samuelson rule (equation (39)) or a deviation from the Samuelson rule (equation (40)). The reason is that the social marginal cost of government spending is still given by $-\frac{1}{A^{i}(s)} U_{N}^{i}(s)$ but not by $U_{C_{N T}}^{i}(s)$ and instead by $\left(1-\tau^{i}(s)\right) U_{C_{N T}}^{i}(s)$. This is because the price of non-traded goods does not reflect the marginal cost of producing them. The discrepancy is precisely given by the labor wedge. The government internalizes this wedge when it decides its consumption of non-traded goods, but private agents do not. As a result, in recessions when $\tau^{i}(s)>0$, it is optimal to tilt the mix of government and private consumption of non-traded goods in the direction of the former, and the opposite holds true in booms when $\tau^{i}(s)<0 .{ }^{48}$

Having characterized the jointly optimal use of international transfers and government spending and shown the robustness of our characterization of optimal international transfers to the availability of government spending as an additional instrument, we now compare the relative performance of government spending and international transfers in a few enlightening limit cases. We first treat the case of the closed-economy limit. We show that international transfers achieve perfect macroeconomic stabilization, with no residual role for government spending. By contrast, in the perfectly open economy limit, international transfers are not used for macroeconomic stabilization, but government spending is. We then treat the cases where the disutility of labor is linear or government spending is purely wasteful. In both cases, we show that even though the optimum is away from the first best, government spending is not useful for macroeconomic stabilization.

\footnotetext{
${ }^{48}$ This analysis assumes that prices are entirely rigid. If there is some adjustment in prices, then increases in government spending stimulate inflation. Given a fixed exchange rate, and other things equal, this leads to an appreciation of the real exchange rate which depresses private spending on non-traded goods and counteracts the direct effect of government spending on total spending on non-traded goods (see e.g. Farhi-Werning 2012). This lessens the macroeconomic stabilization role of government spending. The same holds true for international transfers, as we emphasize in Section 5.
} 
Closed-economy limit. Consider first the closed-economy limit. This limit can be understood as follows. Suppose for simplicity that preferences are given by $v\left(G_{N T}^{i}(s)\right)+\frac{C^{i}(s)^{1-\gamma}}{1-\gamma}-\phi\left(N^{i}(s)\right)$ where $C^{i}(s)=\left[(1-\alpha)^{\frac{1}{\eta}} C_{N T}^{i}(s)^{\frac{\eta-1}{\eta}}+\alpha^{\frac{1}{\eta}} C_{T}^{i}(s)^{\frac{\eta-1}{\eta}}\right]^{\frac{\eta}{\eta-1}}$. Then for any $p, \alpha^{i}(p ; s)$ is decreasing in $\alpha$. The closed-economy limit is obtained in the limit by also scaling where $\alpha$ goes to zero and $\alpha^{i}(p ; s)$ goes to infinity as long as we also scale $E_{T}^{i}(s)$ by $\frac{\alpha}{(1-\alpha)}$ so as to keep $\alpha^{i}(p ; s) E_{T}^{i}(s)$ constant. In this limit, the first-best level of welfare is achieved. This is because international transfers are extremely powerful in relatively closed economies. Indeed, we have already emphasized that the "dollar-for-dollar" output multiplier of transfers is precisely given by the relative expenditure share of non-traded to traded goods. And this multiplier goes to infinity in the closed-economy limit. As we approach the closed-economy limit, vanishingly small departures (as a fraction of each country's nominal income) from the international transfers that support the first-best allocation are enough to perfectly stabilize the economy and deliver $\tau^{i}(s)=0$ for all $i$ and $s$. There is no residual macroeconomic stabilization role for government spending, which then simply follows the first-best Samuelson rule.

Perfectly-open economy limit. The relative usefulness of international transfers and government spending is reversed in the limit where countries are perfectly open, which we capture by letting $\alpha$ go to one. In this limit, international transfers are not used for macroeconomic stabilization, in the sense that constrained efficient and privately optimal risk sharing coincide, so that optimal international transfers are only needed when markets are incomplete, in order to replicate the complete markets allocation with privately optimal risk sharing. By contrast, government spending is used for macroeconomic stabilization as characterized by the same optimality conditions (39) and (40).

Linear disutility of labor. Suppose now that the disutility from labor is linear. We maintain the same parametrization of preferences and assume in addition that $\phi\left(N^{i}(s)\right)=\phi N^{i}(s)$ for some constant $\phi>0$. In this case, the first-order condition for optimal government spending (39) becomes $v^{\prime}\left(G_{N T}^{i}(s)\right)=\frac{\phi}{A^{i}(s)}$. This formula, which pins down $G_{N T}^{i}(s)$ as a function of $A^{i}(s)$, holds both under rigid prices and fixed exchange rates, and under flexible prices or flexible exchange rates. Hence there is a sense in which government spending is not used for macroeconomic stabilization, despite the fact that macroeconomic stabilization is imperfect. The same is not true of international transfers.

Purely wasteful government spending. Another enlightening case is the case in which government spending is purely wasteful, so that it does not enter preferences. ${ }^{49}$ In that case, formulas (39) and (40) indicate that it is optimal not to use government spending, both with rigid prices and fixed exchange rates and with flexible prices or flexible exchange rates. Hence, once again, there is a sense in which government spending is not used for macroeconomic stabilization, despite the fact that macroeconomic stabilization is imperfect. The same is not true of international transfers.

${ }^{49}$ Formally, this means that $U^{i}\left(G_{N T}^{i}(s), C_{N T}^{i}(s), C_{T}^{i}(s), N^{i}(s) ; s\right)$ is independent of $G_{N T}^{i}(s)$. 


\section{Online Appendix: Proofs and Details for Dynamic Model in Sections 4-5}

\section{C.1 Nonlinear Calvo Price Setting Equations}

The equilibrium conditions for the Calvo price setting model can be expressed as follows

$$
\begin{gathered}
\frac{1-\delta \Pi_{H, t}^{\epsilon-1}}{1-\delta}=\left(\frac{F_{t}}{K_{t}}\right)^{\epsilon-1}, \\
K_{t}=\frac{\varepsilon}{\varepsilon-1} \frac{1+\tau^{L}}{A_{H, t}} Y_{t} N_{t}^{\phi}+\delta \beta \Pi_{H, t+1}^{\epsilon} K_{t+1}, \\
F_{t}=Y_{t} C_{t}^{-\sigma} S_{t}^{-1} \mathcal{Q}_{t}+\delta \beta \Pi_{H, t+1}^{\epsilon-1} F_{t+1},
\end{gathered}
$$

together with an equation determining the evolution of price dispersion

$$
\Delta_{t}=h\left(\Delta_{t-1}, \Pi_{H, t}\right),
$$

where $h(\Delta, \Pi)=\delta \Delta \Pi^{\epsilon}+(1-\delta)\left(\frac{1-\delta \Pi^{\epsilon-1}}{1-\delta}\right)^{\frac{\epsilon}{\epsilon-1}}$

\section{C.2 Decomposing the Planning Problem (22)}

We can break down the planning problem into two parts. First, there is an aggregate planning problem determining the average output gap and inflation $\hat{y}_{t}^{*}$ and $\pi_{t}^{*}$

$$
\min \frac{1}{2} \int_{0}^{\infty} e^{-\rho t}\left[\alpha_{\pi}\left(\pi_{t}^{*}\right)^{2}+\left(\hat{y}_{t}^{*}\right)^{2}\right] d t
$$

subject to (27).

Second, there is a disaggregated planning problem determining deviations from the aggregates for output gap, home inflation and consumption smoothing, $\hat{\bar{y}}_{t}^{i}, \hat{\bar{\pi}}_{H, t}^{i}$ and $\hat{\theta}_{t}^{i}$

$$
\min \frac{1}{2} \int_{0}^{\infty} \int_{0}^{1} e^{-\rho t}\left[\alpha_{\pi}\left(\hat{\bar{\pi}}_{H, t}^{i}\right)^{2}+\left(\hat{\bar{y}}_{t}^{i}\right)^{2}+\alpha_{\theta}\left(\hat{\bar{\theta}}^{i}\right)^{2}\right] d i d t
$$

subject to (23), (24), (25), (26). Note that because the forcing variables in this linear quadratic problem satisfy $\int_{0}^{1} \tilde{s}_{t}^{i} d i=0$, the aggregation constraint (26) is not binding. We can therefore drop it from the planning problem. The resulting relaxed planning problem can be broken down into separate component planning problems for each country $i \in[0,1]$

$$
\min \frac{1}{2} \int_{0}^{\infty} e^{-\rho t}\left[\alpha_{\pi}\left(\hat{\bar{\pi}}_{H, t}^{i}\right)^{2}+\left(\hat{\bar{y}}_{t}^{i}\right)^{2}+\alpha_{\theta}\left(\hat{\bar{\theta}}^{i}\right)^{2}\right] d t
$$


subject to (23), (24) and (25).

\section{C.3 Incomplete Markets and No Transfers in a Currency Union}

Here we analyze the solution with incomplete markets and no transfers. This solution imposes $\hat{\theta}^{i}=0$ and coincides with the solution with complete markets and no interventions in financial markets, a well-known property of the Cole-Obstfeld case, where the lack of complete markets is not a constraint on private risk sharing.

Using the fact that $\int_{0}^{1} \hat{\bar{y}}_{t}^{i} d i=\int_{0}^{1} \hat{\bar{\pi}}_{H, t}^{i} d i=0$, we are led to the following planning problem:

$$
\min \frac{1}{2} \int_{0}^{\infty} \int_{0}^{1} e^{-\rho t}\left[\alpha_{\pi}\left(\hat{\bar{\pi}}_{H, t}^{i}\right)^{2}+\left(\hat{\bar{y}}_{t}^{i}\right)^{2}+\alpha_{\pi}\left(\pi_{t}^{*}\right)^{2}+\left(\hat{y}_{t}^{*}\right)^{2}\right] d i d t
$$

subject to

$$
\begin{gathered}
\dot{\bar{\pi}}_{H, t}^{i}=\rho \hat{\bar{\pi}}_{H, t}^{i}-\kappa_{y} \hat{\bar{y}}_{t}^{i} \\
\dot{\bar{y}}_{t}^{i}=-\hat{\bar{\pi}}_{H, t}^{i}-\dot{\tilde{s}}_{t}^{i} \\
\hat{\bar{y}}_{0}^{i}=-\tilde{s}_{0}^{i} \\
\dot{\pi}_{t}^{*}=\rho \pi_{t}^{*}-\kappa_{y} \hat{y}_{t}^{*},
\end{gathered}
$$

where the minimization is over the variables $\hat{\bar{\pi}}_{H, t}^{i}, \pi_{t}^{*}, \hat{y}_{t}^{i}, \hat{y}_{t}^{*}$. Note that since $\hat{\theta}^{i}=0$, the two aggregation constraints $\int_{0}^{1} \hat{\hat{y}}_{t}^{i} d i=0$ and $\int_{0}^{1} \hat{\pi}_{H, t}^{i} d i=0$ are automatically verified.

The solution of the planning problem is then simply $\hat{y}_{t}^{*}=\pi_{t}^{*}=0$ for the aggregates. This result is a restatement of the result in Benigno (2004) and Gali and Monacelli (2008) that optimal monetary policy in a currency union ensures that the union average output gap and inflation are zero in every period. Monetary policy can be chosen at the union level so that monetary conditions are adapted to the average country. The disaggregated variables $\hat{\bar{\pi}}_{H, t}^{i}$ and $\hat{\bar{y}}_{t}^{i}$ solve the following system of differential equations,

$$
\begin{aligned}
\dot{\hat{\pi}}_{H, t}^{i} & =\rho \hat{\bar{\pi}}_{H, t}^{i}-\kappa_{y} \hat{\hat{y}}_{t}^{i}, \\
\dot{\hat{y}}_{t}^{i} & =-\hat{\bar{\pi}}_{H, t}^{i}-\dot{\tilde{s}}_{t}^{i},
\end{aligned}
$$

with initial condition

$$
\hat{\bar{y}}_{0}^{i}=-\tilde{s}_{0}^{i} .
$$

Proposition 18. The solution with incomplete markets and no interventions in financial markets (N $\hat{\bar{F}} A_{0}^{i}=$ $\left.\hat{\theta}^{i}=0\right)$ coincides with the solution with complete markets and no interventions in financial markets. In both cases, union-wide aggregates are zero

$$
\hat{y}_{t}^{*}=\pi_{t}^{*}=0 .
$$




\section{C.4 Transfer Multipliers in a Currency Union}

Before solving the normative problem it is useful to review the positive effects of transfers. The next proposition characterizes the response of the economy to a marginal increase in transfers.

Proposition 19 (Transfer Multipliers). Let $v=\frac{\rho-\sqrt{\rho^{2}+4 \kappa_{y}}}{2}$. Transfer multipliers are given by

$$
\begin{aligned}
\frac{\partial \hat{\bar{y}}_{t}^{i}}{\partial N \hat{\bar{F}} A_{0}^{i}} & =e^{v t} \rho \frac{1-\alpha}{\alpha}-\left(1-e^{\nu t}\right) \rho \frac{1}{1+\phi^{\prime}} \\
\frac{\partial \hat{\bar{\pi}}_{H, t}^{i}}{\partial N \hat{\bar{F}} A_{0}^{i}} & =-v e^{\nu t}\left[\rho \frac{1-\alpha}{\alpha}+\rho \frac{1}{1+\phi}\right], \\
\frac{\partial \hat{\bar{s}}_{t}^{i}}{\partial N \hat{\bar{F}} A_{0}^{i}} & =-\left[1-e^{\nu t}\right]\left[\rho \frac{1-\alpha}{\alpha}+\rho \frac{1}{1+\phi}\right] .
\end{aligned}
$$

The presence of the discount factor $\rho$ in all these expressions is natural because what matters is the annuity value $\rho N \hat{\bar{F}} A_{0}^{i}$ of the transfer. Note that the terms of trade gap equals accumulated inflation: $\hat{\bar{s}}_{t}=-\int_{0}^{t} \hat{\bar{\pi}}_{H, s}^{i} d s$.

Transfers have opposite effects on output in the short and long run. In the short run, when prices are rigid, there is a Keynesian effect due to the fact that transfers stimulate the demand for home goods: $\frac{\partial \hat{y}_{0}^{i}}{\partial N \hat{\bar{F}} A_{0}^{i}}=\rho \frac{1-\alpha}{\alpha}$. In the long run, when prices adjust, the neoclassical wealth effect on labor supply lowers output: $\lim _{t \rightarrow \infty} \frac{\partial \hat{y}_{t}^{i}}{\partial N \hat{\hat{F}} A_{0}^{i}}=-\rho \frac{1}{1+\phi}$. In the medium run, the speed of adjustment, from the Keynesian short-run response to the neoclassical long-run response, is controlled by the degree of price flexibility $\kappa_{y}$, which affects $v^{50}$

Note that the determinants of the Keynesian and neoclassical wealth effects are very different. The strength of the Keynesian effect hinges on the relative expenditure share of home goods $\frac{1-\alpha}{\alpha}$ : the more closed the economy, the larger the Keynesian effect. The strength of the neoclassical wealth effect depends on the elasticity of labor supply $\phi$ : the more elastic labor supply, the larger the neoclassical wealth effect.

Positive transfers also increase home inflation. The long-run cumulated response in the price of home produced goods equals $\rho \frac{1-\alpha}{\alpha}+\rho \frac{1}{1+\phi}$. The first term $\rho \frac{1-\alpha}{\alpha}$ comes from the fact that transfers increase the demand for home goods, due to home bias. The second term $\rho \frac{1}{1+\phi}$ is due to a neoclassical wealth effect that reduces labor supply, raising the wage. How fast this increase in the price of home goods occurs depends positively on the flexibility of prices through its effect on $v .^{51}$

The effects echo the celebrated Transfer Problem controversy of Keynes (1929) and Ohlin (1929). With home bias, a transfer generates a boom when prices are sticky, and a real appreciation of the terms of trade when prices are flexible. The neoclassical wealth effect associated with a transfer comes into play when prices are flexible, and generates an output contraction and a further real

\footnotetext{
${ }^{50}$ Note that $v$ is decreasing in $\kappa_{y}$, with $v=0$ when prices are rigid $\left(\kappa_{y}=0\right)$, and $v=-\infty$ when prices are flexible $\left(\kappa_{y}=\infty\right)$.

${ }^{51}$ Recall that $v$ is decreasing in the degree of price flexibility $\kappa_{y}$.
} 
appreciation.

\section{C.5 Proof of Proposition 14}

In this case, $\kappa_{y}=0$ and the constraint set boils down to $\hat{\bar{y}}_{t}^{i}=(1-\alpha) \hat{\bar{\theta}}^{i}-\tilde{s}_{t}^{i}$, and we are therefore left with the following component planning problem

$$
\min \frac{1}{2} \int_{0}^{\infty} e^{-\rho t}\left[\left((1-\alpha) \hat{\bar{\theta}}^{i}-\tilde{s}_{t}^{i}\right)^{2}+\alpha_{\theta}\left(\hat{\bar{\theta}}^{i}\right)^{2}\right] d t
$$

The result follows.

\section{C.6 Proof of Proposition 19}

We use the decomposition of the planning problem given in Appendix C.2. We focus on the component planning problem for a country $i$. We first solve the behavior of an economy for a given transfer $\hat{\bar{\theta}}^{i}$. Then in Appendix C.7, we solve for the optimal $\hat{\bar{\theta}}^{i}$.

$$
\begin{aligned}
\dot{\overline{\bar{\pi}}}_{H, t}^{i} & =\rho \hat{\bar{\pi}}_{H, t}^{i}-\kappa_{y} \hat{\bar{y}}_{t}^{i}-\lambda \alpha \hat{\bar{\theta}}^{i}, \\
\dot{\hat{y}}_{t}^{i} & =-\hat{\bar{\pi}}_{H, t}^{i}-\dot{\tilde{s}}_{t}^{i}, \\
\hat{\bar{y}}_{0}^{i} & =(1-\alpha) \hat{\bar{\theta}}^{i}-\tilde{s}_{0}^{i} .
\end{aligned}
$$

Define $E_{1}=[1,0]^{\prime}$ and $E_{2}=[0,1]^{\prime}$. Let $X_{t}^{i}=\left[\hat{\bar{\pi}}_{H, t}^{i}, \hat{\bar{y}}_{t}^{i}\right]^{\prime}, B_{t}^{i}=\left[-\lambda \alpha \hat{\bar{\theta}}^{i},-\dot{\tilde{s}}_{t}^{i}\right]^{\prime}=-\lambda \alpha \hat{\bar{\theta}}^{i} E_{1}-\dot{\tilde{s}}_{t}^{i} E_{2}$. Define $A=\left[\begin{array}{cc}\rho & -\kappa_{y} \\ -1 & 0\end{array}\right]$. Let $v=\frac{\rho-\sqrt{\rho^{2}+4 \kappa_{y}}}{2}<0$ be the (only) negative eigenvalue of $A$, and $X_{v}=[-v, 1]^{\prime}$ and be an eigenvector associated with the negative eigenvalue of $A$. The solution is given by

$$
X_{t}^{i}=e^{v t} \alpha_{v}^{i} X_{v}-\int_{t}^{\infty} e^{A(t-s)} B_{s}^{i} d s=e^{v t} \alpha_{v}^{i} X_{v}+\lambda \alpha \hat{\bar{\theta}}^{i} A^{-1} E_{1}+\int_{t}^{\infty} \dot{\tilde{s}}_{u}^{i} e^{A(t-u)} E_{2} d u
$$

where

$$
\begin{gathered}
X_{0}^{i}+\int_{0}^{\infty} e^{-A s} B_{s}^{i} d s=\alpha_{v}^{i} X_{v} \\
E_{2}^{\prime} X_{0}^{i}=(1-\alpha) \hat{\bar{\theta}}^{i}-\tilde{s}_{0}^{i} .
\end{gathered}
$$

We find

$$
\alpha_{v}^{i}=\left[(1-\alpha)-\lambda \alpha E_{2}^{\prime} A^{-1} E_{1}\right] \hat{\bar{\theta}}^{i}-\tilde{s}_{0}^{i}-\int_{0}^{\infty} \dot{\tilde{s}}_{t}^{i} E_{2}^{\prime} e^{-A t} E_{2} d t
$$

Using $E_{2}^{\prime} A^{-1} E_{1}=-\kappa_{y}^{-1}$, and $E_{1}^{\prime} A^{-1} E_{1}=0$, we can infer the path for output $\hat{\bar{y}}_{t}^{i}=E_{2}^{\prime} X_{t}^{i}$ and inflation $\hat{\bar{\pi}}_{H, t}^{i}=E_{1}^{\prime} X_{t}^{i}$ as follows:

$$
\hat{\bar{y}}_{t}^{i}=e^{v t} \alpha_{v}^{i}-\frac{\lambda}{\kappa_{y}} \alpha \hat{\bar{\theta}}^{i}+\int_{t}^{\infty} \dot{\tilde{s}}_{u}^{i} E_{2}^{\prime} e^{A(t-u)} E_{2} d u
$$




$$
\hat{\bar{\pi}}_{H, t}^{i}=-v e^{v t} \alpha_{v}^{i}+\int_{t}^{\infty} \dot{\tilde{s}}_{u}^{i} E_{1}^{\prime} e^{A(t-u)} E_{2} d u .
$$

The results in Proposition 19 follow by specializing these expressions to the case $\tilde{s}_{t}=0$.

\section{C.7 Derivation of the Optimum in Section 6.2}

In Appendix C.6, we solved for the behavior of the disaggregated variables $X_{t}^{i}=\left[\hat{\bar{\pi}}_{H, t}^{i}, \hat{\bar{y}}_{t}^{i}\right]^{\prime}$ for a given $\hat{\bar{\theta}}^{i}$. We now solve for the optimal $\hat{\bar{\theta}}^{i}$. We apply the results of Appendix C.6 in the particular case $\tilde{s}_{t}^{i}=\tilde{s}_{0}^{i} e^{-\psi t}$. We get

$$
X_{t}^{i}=e^{v t} \alpha_{v}^{i} X_{v}+\lambda \alpha \hat{\bar{\theta}}^{i} A^{-1} E_{1}-\psi e^{-\psi t} \tilde{s}_{0}^{i}(A+\psi I)^{-1} E_{2}
$$

where

$$
\alpha_{v}^{i}=\left[(1-\alpha)-\lambda \alpha E_{2}^{\prime} A^{-1} E_{1}\right] \hat{\theta}^{i}-\tilde{s}_{0}^{i}+\psi \tilde{s}_{0}^{i} E_{2}^{\prime}(A+\psi I)^{-1} E_{2}
$$

$E_{1}=[1,0]^{\prime}, E_{2}=[0,1]^{\prime}, A=\left[\begin{array}{cc}\rho & -\kappa_{y} \\ -1 & 0\end{array}\right], v=\frac{\rho-\sqrt{\rho^{2}+4 \kappa_{y}}}{2}<0$ is the negative eigenvalue of $A$, and $X_{v}=[-v, 1]^{\prime}$ is an eigenvector associated with the negative eigenvalue of $A$. This yields

$$
\begin{aligned}
\hat{\bar{\pi}}_{H, t}^{i} & =-\alpha_{v} v e^{v t}-\frac{\psi \kappa_{y} \tilde{s}_{0}^{i}}{(\rho+\psi) \psi-\kappa_{y}} e^{-\psi t}, \\
\hat{\bar{y}}_{t}^{i} & =\alpha_{v} e^{v t}-\frac{\alpha \lambda}{\kappa_{y}} \hat{\bar{\theta}}^{i}-\frac{(\rho+\psi) \psi \tilde{s}_{0}^{i}}{(\rho+\psi) \psi-\kappa_{y}} e^{-\psi t} .
\end{aligned}
$$

We need to solve

$$
\min _{\hat{\bar{\theta}}^{i}} \frac{1}{2} \frac{\alpha_{\theta}}{\rho}\left(\hat{\bar{\theta}}^{i}\right)^{2}+\frac{1}{2} \int_{0}^{\infty} e^{-\rho t}\left[\alpha_{\pi}\left(\hat{\bar{\pi}}_{H, t}^{i}\right)^{2}+\left(\hat{\bar{y}}_{t}^{i}\right)^{2}\right] d t
$$

This can be rewritten as

$$
\begin{aligned}
\min _{\hat{\bar{\theta}}^{i}} \frac{1}{2} \frac{\alpha_{\theta}}{\rho}\left(\hat{\bar{\theta}}^{i}\right)^{2}+\frac{1}{2} \int_{0}^{\infty} e^{-\rho t} & {\left[\alpha_{\pi}\left(\alpha_{v}^{2} v^{2} e^{2 v t}+\left(\frac{\psi \kappa_{y} \tilde{s}_{0}^{i}}{(\rho+\psi) \psi-\kappa_{y}}\right)^{2} e^{-2 \psi t}+\frac{2 \alpha_{v} \nu \psi \kappa_{y} \tilde{s}_{0}^{i}}{(\rho+\psi) \psi-\kappa_{y}} e^{-(\psi-v) t}\right)\right.} \\
& +\left(\alpha_{v}^{2} e^{2 v t}+\left(\frac{\alpha \lambda}{\kappa_{y}}\right)^{2}\left(\hat{\bar{\theta}}^{i}\right)^{2}+\left(\frac{(\rho+\psi) \psi \tilde{s}_{0}^{i}}{(\rho+\psi) \psi-\kappa_{y}}\right)^{2} e^{-2 \psi t}-2 \alpha_{v} \frac{\alpha \lambda}{\kappa_{y}} \hat{\bar{\theta}}^{i} e^{v t}\right. \\
& \left.\left.-\frac{2 \alpha_{v}(\rho+\psi) \psi \tilde{s}_{0}^{i}}{(\rho+\psi) \psi-\kappa_{y}} e^{-(\psi-v) t}+\frac{2 \alpha \lambda(\rho+\psi) \psi \tilde{s}_{0}^{i}}{\kappa_{y}\left[(\rho+\psi) \psi-\kappa_{y}\right]} \hat{\bar{\theta}}^{i} e^{-\psi t}\right)\right] d t .
\end{aligned}
$$

Solving the integrals, we arrive at

$$
\begin{aligned}
\min _{\hat{\theta}^{i}} & \frac{\alpha_{\theta}}{2 \rho}\left(\hat{\bar{\theta}}^{i}\right)^{2}+\frac{(\alpha \lambda)^{2}}{2 \rho \kappa^{2}}\left(\hat{\bar{\theta}}^{i}\right)^{2}+\frac{\alpha_{\pi} v^{2}+1}{2(\rho-2 v)}\left(\alpha_{v}^{i}\right)^{2}+\frac{\left(\alpha_{\pi} \kappa^{2}+(\rho+\psi)^{2}\right)\left(\psi \tilde{s}_{0}^{i}\right)^{2}}{2(\rho+2 \psi)\left[(\rho+\psi) \psi-\kappa_{y}\right]^{2}} \\
& +\frac{\left(\alpha_{\pi} v \kappa_{y}-\rho-\psi\right) \psi \tilde{s}_{0}^{i}}{(\rho+\psi-v)\left[(\rho+\psi) \psi-\kappa_{y}\right]} \alpha_{v}^{i}+\frac{\alpha \lambda \tilde{s}_{0}^{i} \psi}{(\rho+\psi) \psi \kappa_{y}-\kappa_{y}^{2}} \hat{\bar{\theta}}^{i}-\frac{\alpha \lambda}{(\rho-v) \kappa_{y}} \alpha_{v}^{i} \hat{\bar{\theta}}^{i} .
\end{aligned}
$$


The solution is

$$
\hat{\theta}^{i}=\frac{\left[\frac{\alpha \lambda}{(\rho-v) \kappa_{y}}-\frac{\alpha_{\pi} \nu^{2}+1}{\rho-2 v}\left(1-\alpha+\frac{\alpha \lambda}{\kappa_{y}}\right)\right] \frac{\kappa_{y} \tilde{s}_{0}^{i}}{(\rho+\psi) \psi-\kappa_{y}}-\frac{\alpha \lambda \psi \tilde{s}_{0}^{i}}{(\rho+\psi) \psi \kappa_{y}-\kappa_{y}^{2}}-\frac{\left(\alpha_{\pi} v \kappa_{y}-\rho-\psi\right) \psi \tilde{s}_{0}^{i}\left(1-\alpha+\frac{\alpha \lambda}{\kappa_{y}}\right)}{(\rho+\psi-v)\left((\rho+\psi) \psi-\kappa_{y}\right)}}{\frac{\alpha_{\theta}}{\rho}+\frac{(\alpha \lambda)^{2}}{\rho \kappa_{y}^{2}}+\frac{\alpha_{\pi} v^{2}+1}{\rho-2 v}\left(1-\alpha+\frac{\alpha \lambda}{\kappa_{y}}\right)^{2}-\frac{2 \alpha \lambda}{(\rho-\nu) \kappa_{y}}\left(1-\alpha+\frac{\alpha \lambda}{\kappa_{y}}\right)} .
$$

\section{C.8 Proof of Proposition 15}

In the closed-economy limit, as $\alpha \rightarrow 0$, we have $\alpha_{\theta}=0$, and we see directly from Section C.7 that

$$
\hat{\bar{\theta}}^{i}=-\frac{\kappa_{y} \tilde{s}_{0}^{i}}{(\rho+\psi) \psi-\kappa_{y}}-\frac{\rho-2 v}{\alpha_{\pi} v^{2}+1} \frac{\left(\alpha_{\pi} v \kappa_{y}-\rho-\psi\right) \psi \tilde{s}_{0}^{i}}{(\rho+\psi-v)\left[(\rho+\psi) \psi-\kappa_{y}\right]} .
$$

\section{Online Appendix: Extensions of Dynamic Model in Sections 4-5 for Section 6}

In this appendix, we detail two extensions of the dynamic model by introducing hand-to-mouth consumers and alternative macroeconomic instruments. This appendix is organized as follows. Section D.1 outlines the extended model. Section D.2 derives the allocations given exogenous policies. Section D.3 derives the loss function. Sections D.4-D.9 set up the planning problem as well as the solution method for alternative macroeconomic instruments: international transfers, capital controls, government spending, redistribution, deficits, and then all domestic fiscal policy instruments combined. Section D.10 specializes the previous sections to the case of no hand-to-mouth consumers. We first setup up the model allowing only for international transfers, government spending, and capital controls in Sections D.4-D.6. We then generalize it to allow for redistribution and deficits in Sections D.7-D.9.

\section{D.1 Model}

This is a model of fiscal unions with two types of agents: hand-to-mouth (HtM) agents of measure $\chi \in[0,1]$ and optimizers of measure $1-\chi$. We assume throughout that countries are in a currency union. From the get go, we study idiosyncratic productivity shocks in a given country $i$, which will correspond to Home in the exposition of Sections 4-5. ${ }^{52}$ We abstract from idiosyncratic shocks in other countries and from aggregate shocks. As a result, union-wide aggregates and policies are constant at their steady state where outputs in all countries are equal to one.

\footnotetext{
${ }^{52}$ There is no rest of the world (RoW) beyond the members of the fiscal union.
} 


\section{D.1.1 Households}

The two types of households in country $i$ share the same preferences, given by

$$
\int_{0}^{\infty} e^{-\rho t}\left((1-v) \ln \left(C_{t}^{i, s}\right)-\frac{1}{1+\phi}\left(N_{t}^{i, s}\right)^{1+\phi}+v \ln \left(G_{t}^{i}\right)\right) d t
$$

where $s \in\{o, r\}$ designates the agent type; $o$ denotes optimizers and $r$ HtM agents. As in the main paper, $N_{t}^{i, s}$ denotes labor supplied by agent $s$ in country $i$, and the consumption basket is given by

$$
C_{t}^{i, s}=\left[(1-\alpha)^{\frac{1}{\eta}}\left(C_{H, t}^{i, s}\right)^{\frac{\eta-1}{\eta}}+\alpha^{\frac{1}{\eta}}\left(C_{F, t}^{i, s}\right)^{\frac{\eta-1}{\eta}}\right]^{\frac{\eta}{\eta-1}},
$$

where

$$
C_{H, t}^{i, s}=\left(\int_{0}^{1}\left(C_{H, t}^{i, s}(j)\right)^{\frac{\epsilon-1}{\epsilon}} d j\right)^{\frac{\epsilon}{\epsilon-1}}
$$

is the basket of home goods consumed by agent $s$ in country $i$, with $j$ denoting an individual good variety. Similarly,

$$
C_{F, t}^{i, s}=\left(\int_{0}^{1}\left(C_{k, t}^{i, s}\right)^{\frac{\gamma-1}{\gamma}} d k\right)^{\frac{\gamma}{\gamma-1}}
$$

denotes the consumption index of imported goods consumed by agent $s$. We also have

$$
C_{k, t}^{i, s}=\left(\int_{0}^{1}\left(C_{k, t}^{i, s}(j)\right)^{\frac{\epsilon-1}{\epsilon}} d j\right)^{\frac{\epsilon}{\epsilon-1}}
$$

Throughout the paper, we focus exclusively on the Cole-Obstfeld calibration and subsequently set $\eta=\gamma=1$. As is standard, the parameter $\alpha$ indexes the degree of home bias in goods consumption. As $\alpha \rightarrow 0$, the share of imported goods in domestic consumption vanishes. As $\alpha \rightarrow 1$, the share of home goods vanishes. The two agent types differ with respect to their per-period budget constraints, which we now describe.

\section{D.1.2 Budget Constraints, Transfers, and NFA}

In our analysis of transfers, country $i$ will receive a transfer in every period from the remaining members of the fiscal union after the realization of a productivity shock. We impose that each optimizer and each HtM agent receive an equal share of this transfer in every period. To that end, the government will tax optimizers and rebate the proceeds to the HtM agents accordingly.

For optimizers, only the net present value of this transfer matters. We encode the net present value of this transfer in the initial net foreign asset position of country $i, N F A_{0}^{i}$, which we will later derive from the optimizers' budget constraint explicitly.

We start our discussion of transfers by considering the optimizers' budget constraint. Each opti- 
mizer faces the per-period constraint of

$$
\begin{array}{r}
\int_{0}^{1} P_{H, t}^{i}(j) C_{H, t}^{i, o}(j) d j+\int_{0}^{1} \int_{0}^{1} P_{t}^{k}(j) C_{k, t}^{i}(j) d k d j+D_{i, t+1}^{i}+\int_{0}^{1} D_{k, t+1}^{i} d k \\
\leq W_{t}^{i} N_{t}^{i, o}+\left(1+i_{t-1}^{i}\right) D_{i, t}^{i}+\int_{0}^{1}\left(1+\tau_{t-1}^{c a p, i}\right)\left(1+i^{*}\right) D_{k, t}^{i} d k+\frac{1}{1-\chi} P_{t}^{i} Z_{H, t}^{i}+P_{t}^{i} T_{t}^{c a p, i, o}+P_{t}^{i} T_{t}^{i, o}+P_{t}^{i} \mathcal{T}_{t}^{i}
\end{array}
$$

In this expression, $D_{k, t+1}^{i}$ is country $i^{\prime}$ s holdings of country $k$ bonds, $D_{i, t+1}^{i}$ is country $i^{\prime}$ s holdings of country $i$ bonds, $P_{t}^{i}$ is the domestic consumer price index, $W_{t}^{i}$ is the domestic wage, $P_{H, t}^{i}(j)$ is the price of variety $j$ of the home good in country $i, P_{t}^{k}(j)$ is the price of variety $j$ of the good of country $k$. Besides labor income and income on bond holdings, the budget constraint of optimizers features three additional sources of transfers. We denote by $P_{t}^{i} Z_{H, t}^{i}=P_{H, t}^{i} Y_{t}^{i}-W_{t}^{i} N_{t}^{i}$ nominal domestic profits, where $P_{H, t}^{i}$ is the price of the home good in country $i$. Note that optimizers receive all the profits in the economy.

We now turn to the policy instruments that show up in the budget constraint. $T_{t}^{i, 0}$ is the real transfer each optimizer in country $i$ receives from the domestic government. $\mathcal{T}_{t}^{i}$ denotes the transfer that each optimizer receives from the fiscal union. Note that for convenience, we adopt the convention that international transfers are paid to optimizers and then passed through to HtM consumers via government taxes and transfers. We could equivalently have specified that international transfers are paid to the government and then distributed to optimizers and HtM consumers. Finally $i_{t-1}^{i}$ is the interest rate in country $i$ and $i^{*}$ in the rest of the currency union with $\beta\left(1+i^{*}\right)=1$. These can be different because of capital controls in the form of taxes on capital inflows $\tau_{t-1}^{c a p, i}$ in country $i$. We rebate the proceeds from the capital controls lump sum to optimizers using $T_{t}^{c a p, i, o}=-\int_{0}^{1} \tau_{t-1}^{c a p, i}\left(1+i^{*}\right) D_{k, t}^{i} d k$, and from now on, we omit these flows from the government budget constraint.

The HtM budget constraint given by

$$
P_{t}^{i} C_{t}^{i, r}=W_{t}^{i} N_{t}^{i, r}+P_{t}^{i} T_{t}^{i, r}
$$

In our baseline model (we relax this later), the government must balance its budget in every period $t$, yielding the following government budget constraint

$$
P_{H, t}^{i} G_{t}^{i}+\chi P_{t}^{i} T_{t}^{i, r}+(1-\chi) P_{t}^{i} T_{t}^{i, o}=0,
$$

where $G_{t}^{i}$ are real government purchases of domestic goods.

We impose that the lump sum transfers to optimizers $T_{t}^{i, o}$ and to hand-to-mouth agents $T_{t}^{i, r}$ are such that after these transfers: (i) each agent receives and equal share of domestic profits $P_{t}^{i} Z_{H, t}^{i}$ in every period; (ii) each agent receives and equal share of the international transfer $P_{t}^{i} \mathcal{T}_{t}^{i}$ in every period; (iii) each agent contributes equally towards the financing of domestic government spending $P_{H, t}^{i} G_{t}^{i}$ in every period.

For convenience, we introduce a separate notation, $\tau_{t}^{i, 0}$ and $\tau_{t}^{i, r}$, for the part of these transfers that 
ensures equal distribution of profits across agents as described in point (i) above:

$$
\begin{gathered}
T_{t}^{i, r}-\tau_{t}^{i, r}=Z_{H, t}^{i} \\
T_{t}^{i, o}-\tau_{t}^{i, o}=-\frac{\chi}{1-\chi} Z_{H, t}^{i} .
\end{gathered}
$$

The government budget constraint can then equivalently be restated as

$$
P_{H, t}^{i} G_{t}^{i}+\chi P_{t}^{i} \tau_{t}^{i, r}+(1-\chi) P_{t}^{i} \tau_{t}^{i, o}=0 .
$$

We denote the net present value of international transfers $P_{t}^{i} \mathcal{T}_{t}^{i}$ discounted as the union interest rate $i^{*}=\beta^{-1}-1$ in units of the initial domestic price index, $P_{0}^{i}$, which is the same thing as the price index for imports in country $i, P_{F}^{i}$, in every period: ${ }^{53}$

$$
N F A_{0}^{i}=\sum_{t=0}^{\infty} \beta^{t} \frac{P_{t}^{i} \mathcal{T}_{t}^{i}}{P_{0}^{i}}=\sum_{t=0}^{\infty} \beta^{t} \frac{P_{t}^{i} \mathcal{T}_{t}^{i}}{P_{F}^{i}}=\sum_{t=0}^{\infty} \beta^{t}\left(S_{t}^{i}\right)^{-(1-\alpha)} \mathcal{T}_{t}^{i} .
$$

Points (ii) and (iii) above imply that

$$
N F A_{0}^{i}=\sum_{t=0}^{\infty} \beta^{t}\left[\tau_{t}^{i, r}+\left(S_{t}^{i}\right)^{-\alpha} G_{t}^{i}\right] .
$$

In equilibrium, $N F A_{0}$ is equal to the net present value of trade balances, as required by the current account identity. Net exports are given by

$$
N X_{t}^{i}=P_{H, t}^{i} Y_{t}^{i}-P_{t}^{i} C_{t}^{i}-P_{H, t}^{i} G_{t}^{i} .
$$

Therefore, we have

$$
N F A_{0}^{i}=-\sum_{t=0}^{\infty} \beta^{t} \frac{N X_{t}^{i}}{P_{F}^{i}}=-\sum_{t=0}^{\infty} \beta^{t}\left(\left(S_{t}^{i}\right)^{-1} Y_{t}^{i}-\left(S_{t}^{i}\right)^{-(1-\alpha)} C_{t}^{i}-\left(S_{t}^{i}\right)^{-1} G_{t}^{i}\right) .
$$

\section{D.1.3 Firms}

Our descriptions of firms here is identical to that in the body of the paper. Firms produce differentiated goods with a linear technology given by

$$
Y_{t}^{i}(j)=A_{t}^{i} N_{t}^{i}(j)
$$

where $A_{t}^{i}$ is the productivity in the home country. To offset the monopoly distortion, we introduce a constant employment tax $1+\tau^{L}$ so that the real marginal cost deflated by the home producer price index is given by

$$
M C_{t}^{i}=\frac{1+\tau^{L}}{A_{t}^{i}} \frac{W_{t}^{i}}{P_{H, t}^{i}} .
$$

\footnotetext{
${ }^{53}$ In the absence of transfers we simply have $N F A_{0}^{i}=\mathcal{T}_{t}^{i}=0$ and $\tau_{t}^{i, r}=\tau_{t}^{i, o}=-\left(S_{t}^{i}\right)^{-\alpha} G_{t}^{i}$.
} 
This employment tax is set cooperatively at a symmetric steady state with flexible prices. As we show later, this implies that $\tau^{L}=-\frac{1}{\epsilon}$ or $\frac{\epsilon}{\epsilon-1}\left(1+\tau^{L}\right)=1$.

As in the main paper, we assume that the Law of One Price holds at all times and maintain Producer Currency Pricing. Our price setting assumptions here are also analogous to those in the paper, except that we now use continuous time. As a result, we can write the supply side equilibrium conditions of the model as

$$
\begin{aligned}
\frac{1-\delta\left(\Pi_{H, t}^{i}\right)^{\epsilon-1}}{1-\delta} & =\left(\frac{F_{t}^{i}}{K_{t}^{i}}\right)^{\epsilon-1} \\
K_{t}^{i} & =\frac{\epsilon}{\epsilon-1} \frac{1+\tau^{L}}{A_{t}^{i}} Y_{t}^{i}\left(N_{t}^{i}\right)^{\phi}+\beta \delta\left(\Pi_{H, t+1}^{i}\right)^{\epsilon} K_{t+1}^{i} \\
F_{t}^{i} & =\frac{Y_{t}^{i} Q_{t}^{i}}{C_{t}^{i} S_{t}^{i}}+\beta \delta\left(\Pi_{H, t+1}^{i}\right)^{\epsilon} F_{t+1}^{i} \\
\Pi_{H, t}^{i} & =\frac{P_{H, t}^{i}}{P_{H, t-1}^{i}} .
\end{aligned}
$$

\section{D.1.4 Terms of trade, Exchange rates, and UIP}

We briefly restate here our conventions about price indices and exchange rates from the body of the paper.

We can write the consumer price index (CPI) as

$$
P_{t}^{i}=\left[(1-\alpha)\left(P_{H, t}^{i}\right)^{1-\eta}+\alpha\left(P_{F}^{i}\right)^{1-\eta}\right]^{\frac{1}{1-\eta}}
$$

and the home producer price index (PPI) as

$$
P_{H, t}^{i}=\left(\int_{0}^{1} P_{H, t}^{i}(j)^{1-\epsilon} d j\right)^{\frac{1}{1-\epsilon}} .
$$

The import price index is given by

$$
P_{F}^{i}=\left(\int_{0}^{1}\left(P_{k}^{i}\right)^{1-\gamma} d k\right)^{\frac{1}{1-\gamma}}
$$

with $P_{k}^{i}=\left(\int_{0}^{1} P_{k}^{i}(j)^{1-\epsilon} d j\right)^{\frac{1}{1-\epsilon}}$ denoting country $i^{\prime}$ s PPI.

Under the Law of One Price, we have $P_{H, t}^{i}=P_{H, t}^{k}$ where $P_{H, t}^{k}$ is country $k^{\prime}$ s domestic PPI in terms of country $k^{\prime}$ s currency. Therefore, $P_{F}^{i}=P^{*}$, where $P^{*}=\left(\int_{0}^{1}\left(P_{H}^{k}\right)^{1-\gamma} d k\right)^{\frac{1}{1-\gamma}}$, is the world price index. As is standard, we define the terms of trade by $S_{t}^{i}=\frac{P_{F}^{i}}{P_{H, t}^{i}}$, which allows us to write the home CPI as

$$
P_{t}^{i}=P_{H, t}^{i}\left(1-\alpha+\alpha\left(S_{t}^{i}\right)^{1-\eta}\right)^{\frac{1}{1-\eta}} .
$$


The real exchange rate with country $k$ is given by $Q_{k, t}^{i}=\frac{P^{k}}{P_{t}^{i}}$ where $P^{k}$ is country $k^{\prime} \mathrm{s} C P I$. The effective real exchange rate is therefore $Q_{t}^{i}=\frac{P^{*}}{P_{t}^{i}}$.

The UIP condition is

$$
1+i_{t}^{i}=\left(1+\tau_{t}^{c a p, i}\right)\left(1+i^{*}\right) .
$$

We define the Pareto weights $\Theta_{t}^{i}$ by

$$
C_{t}^{i, o}=\Theta_{t}^{i} C^{*, o} \mathcal{Q}_{t}^{i} .
$$

The UIP condition implies a direct mapping between capital controls and these Pareto weights

$$
\frac{\Theta_{t+1}^{i}}{\Theta_{t}^{i}}=1+\tau_{t}^{c a p, i}
$$

From now on, we simply describe capital controls in terms of the Pareto weights $\Theta_{t}^{i}$.

\section{D.1.5 Steady State}

We postulate a symmetric steady state such that all countries $i \in[0,1]$ within the monetary union are symmetric so that $S^{i}=\Theta^{i}=1$ and for simplicity set $A^{i}=A^{k}=A^{*}=1$. The social planner sets the labor tax $\tau^{L}$ so that the steady state allocation is efficient. In particular, the centralized problem to pin down the efficient steady state allocation can be written as

$$
\max (1-\chi)(1-v) \ln \left(C^{i, o}\right)+\chi(1-v) \ln \left(C^{i, r}\right)-\frac{\chi}{1+\phi}\left(N^{i, r}\right)^{1+\phi}-\frac{1-\chi}{1+\phi}\left(N^{i, o}\right)^{1+\phi}+v \ln \left(G^{i}\right),
$$

subject to technology and resource constraints

$$
\begin{aligned}
Y^{i} & =N^{i} \\
Y^{i} & =C^{i}+G^{i} \\
C^{i} & =\chi C^{i, r}+(1-\chi) C^{i, o} \\
N^{i} & =\chi N^{i, r}+(1-\chi) N^{i, o} .
\end{aligned}
$$

The first-order conditions with respect to $C^{i, o}, C^{i, r}, N^{i, o}$ and $N^{i, r}$ immediately imply that agents should be symmetric. That is, the efficient steady state is characterized by $C^{i, o}=C^{i, r}=C^{i}$ and $N^{i, o}=N^{i, r}=$ $N^{i}$. The remaining first-order conditions can be rearranged so that

$$
\begin{aligned}
(1-v) G^{i} & =v C^{i} \\
\left(N^{i}\right)^{\phi} & =\frac{1-v}{C^{i}} .
\end{aligned}
$$


The first of these, together with the resource constraint, implies that

$$
v=\frac{G^{i}}{Y^{i}}, \quad 1-v=\frac{C^{i}}{Y^{i}} .
$$

The second, using the technology constraint, yields

$$
N^{i}=1, \quad Y^{i}=1
$$

It is straightforward to show, as in Gali-Monacelli (2008) for example, that the efficient steady state can be decentralized with an employment tax that solves $\frac{\epsilon}{\epsilon-1}\left(1+\tau^{L}\right)=1$, which implies $\tau^{L}=-\frac{1}{\epsilon}$.

Let $Z_{H, t}^{i}$ denote profits of domestic firms, defined as

$$
P_{t}^{i} Z_{H, t}^{i}=P_{H, t}^{i} Y_{t}^{i}-W_{t}^{i} N_{t}^{i}
$$

In steady state, we have

$$
Z_{H}^{i}=\left(S^{i}\right)^{-\alpha} Y^{i}-\frac{1}{1-v} C^{i, o}\left(N^{i, o}\right)^{\phi} N^{i}=Y^{i}-(1-v) Y^{i} \frac{1}{1-v}=0,
$$

where the second line follows by setting $S^{i}=1$ and $N^{i, o}=N^{i}=1$.

It follows from our discussion in the previous subsection that in steady state, when trade is balanced and there are no transfers, we have

$$
\tau^{i, r}=\tau^{i, o}=-\left(S^{i}\right)^{-\alpha} G^{i}=-v Y^{i}
$$

which is consistent with the government budget constraint. This furthermore implies that

$$
T^{i, r}=T^{i, o}+\frac{1}{1-\chi} Z_{H}^{i}=T^{i, o}=-v Y^{i} .
$$

\section{D.1.6 Linearized Equilibrium}

As in the baseline model of Sections(4)-(5) and for the same reasons, we move to continuous time.

Notation. We begin by defining some useful notation. For all variables $X_{t}$, we drop the time subscript to denote the steady state by $X$. For $X_{t} \in\left\{T_{t}^{i, o}, T_{t}^{i, r}, \tau_{t}^{i, o}, \tau_{t}^{i, r}, N F A_{0}^{i}, N X_{t}^{i}\right\}$, we define $x_{t}=$ $X_{t}-X$ and $\tilde{x}_{t}=\tilde{X}_{t}-X$, where $\tilde{X}_{t}$ denotes the natural allocation. ${ }^{54}$ For all other variables, we define $x_{t}=\ln \left(X_{t}\right)-\ln (X)$ and $\tilde{x}_{t}=\ln \left(\tilde{X}_{t}\right)-\ln (X)$.

Furthermore, it will be convenient to work with the allocation in gaps from the natural allocation. To that end, we define for all variables $\hat{x}_{t}=x_{t}-\tilde{x}_{t}$. We will furthermore normalize our allocation by writing it in gaps from union aggregates. For all country $i$ variables, we let $\hat{x}_{t}^{i}=\hat{x}_{t}^{i}-\hat{x}_{t}^{*}$, where

\footnotetext{
${ }^{54}$ We will abuse notation and let $\tau_{t}^{i, o}$ and $\tau_{t}^{i, r}$ refer to both the level of rebates and the normalized deviation from steady state. Throughout, it will be obvious which of the two we refer to.
} 
$\hat{x}_{t}^{*}=\int_{0}^{1} \hat{x}_{t}^{i} d i$ is the union aggregate of the log-linearized variable $x_{t}^{i}$ in gaps from the natural. ${ }^{55}$

Finally since inflation under flexible prices is not well defined, we define $\hat{\pi}^{i}=\pi^{i}$ and $\hat{\pi}^{i}=$ $\pi^{i}-\pi^{*}$ for both PPI and CPI inflation.

Labor supply. We start with the households' labor supply condition,

$$
(1-v) \frac{W_{t}^{i}}{P_{t}^{i}}=C_{t}^{i, o}\left(N_{t}^{i, o}\right)^{\phi}=C_{t}^{i, r}\left(N_{t}^{i, r}\right)^{\phi} .
$$

Linearizing, we have

$$
w_{t}^{i}-p_{t}^{i}=c_{t}^{i, o}+\phi n_{t}^{i, o}=c_{t}^{i, r}+\phi n_{t}^{i, r}
$$

Therefore, we have

$$
\begin{aligned}
\text { (Natural) } & \tilde{c}_{t}^{i, o}+\phi \tilde{n}_{t}^{i, o}=\tilde{c}_{t}^{i, r}+\phi \tilde{n}_{t}^{i, r} \\
\text { (Gaps from natural) } & \hat{c}_{t}^{i, o}+\phi \hat{n}_{t}^{i, o}=\hat{c}_{t}^{i, r}+\phi \hat{n}_{t}^{i, r} \\
\text { (Gaps from union) } & \hat{\bar{c}}_{t}^{i, o}+\phi \hat{\bar{n}}_{t}^{i, o}=\hat{\bar{c}}_{t}^{i, r}+\phi \hat{\bar{n}}_{t}^{i, r} .
\end{aligned}
$$

Backus-Smith. We have

$$
C_{t}^{i, o}=\Theta_{t}^{i} C_{t}^{*, o} Q_{t}^{i}
$$

where $Q_{t}^{i}=\left(S_{t}^{i}\right)^{1-\alpha} \cdot{ }^{56}$ Linearizing, we have

$$
c_{t}^{i, o}=\theta_{t}^{i}+c_{t}^{*, o}+(1-\alpha) s_{t}^{i} .
$$

Therefore, we have

(Natural)

(Gaps from natural)

(Gaps from union)

$$
\begin{aligned}
& \tilde{c}_{t}^{i, o}=\tilde{\theta}_{t}^{i}+(1-\alpha) \tilde{s}_{t}^{i} \\
& \hat{c}_{t}^{i, o}=\hat{\theta}_{t}^{i}+c_{t}^{*, o}+(1-\alpha) \hat{s}_{t}^{i} \\
& \hat{\bar{c}}_{t}^{i, o}=\hat{\bar{\theta}}_{t}^{i}+(1-\alpha) \hat{\bar{s}}_{t}^{i} .
\end{aligned}
$$

Aggregation. We have

$$
c_{t}^{i}=\chi c_{t}^{i, r}+(1-\chi) c_{t}^{i, o}
$$

and

$$
n_{t}^{i}=\chi n_{t}^{i, r}+(1-\chi) n_{t}^{i, o}
$$

Production. We have

${ }^{55}$ We note here that under idiosyncratic shocks, we will have $\tilde{x}_{t}^{*}=\int_{0}^{1} \tilde{x}_{t}^{i} d i=0$ for all natural variables. That is, any idiosyncratic shock to measure- 0 country $i$ will not affect the union's natural allocation.

${ }^{56} C_{t}^{*, o}=\int_{0}^{1} C_{t}^{i, o} d i$ denotes the union aggregate of optimizers' consumption. For double-superscripts (country and agent type), we write the country index ( $i$ or $*$ ) first, followed by the agent index ( $o$ or $r$ ). 


$$
Y_{t}^{i}=A_{t}^{i} N_{t}^{i}
$$

Linearizing, we have

$$
y_{t}^{i}=a_{t}^{i}+n_{t}^{i}
$$

Therefore, we have

$$
\begin{aligned}
\text { (Natural) } & \tilde{y}_{t}^{i}=a_{t}^{i}+\tilde{n}_{t}^{i} \\
\text { (Gaps from natural) } & \hat{y}_{t}^{i}=\hat{n}_{t}^{i} \\
\text { (Gaps from union) } & \hat{\bar{y}}_{t}^{i}=\hat{\bar{n}}_{t}^{i} .
\end{aligned}
$$

HtM budget constraint. We have

$$
P_{t}^{i} T_{t}^{i, r}+W_{t}^{i} N_{t}^{i, r}=P_{t}^{i} C_{t}^{i, r},
$$

or

$$
T_{t}^{i, r}+\frac{1}{1-v} C_{t}^{i, r}\left(N_{t}^{i, r}\right)^{1+\phi}=C_{t}^{i, r},
$$

Linearizing, we have

$$
\frac{T^{i, r}}{Y^{i}}+t_{t}^{i, r}+(1+\phi) n_{t}^{i, r}+v c_{t}^{i, r}+v=0,
$$

and since $\frac{T^{i, r}}{Y^{i}}=-v$, we have

$$
t_{t}^{i, r}+(1+\phi) n_{t}^{i, r}+v c_{t}^{i, r}=0
$$

Therefore, we have

$$
\begin{aligned}
\text { (Natural) } & \tilde{\hat{t}}_{t}^{i, r}+(1+\phi) \tilde{n}_{t}^{i, r}+v \tilde{c}_{t}^{i, r}=0 \\
\text { (Gaps from natural) } & \hat{t}_{t}^{i, r}+(1+\phi) \hat{n}_{t}^{i, r}+v \hat{c}_{t}^{i, r}=0 \\
\text { (Gaps from union) } & \hat{\hat{t}}_{t}^{i, r}+(1+\phi) \hat{\bar{n}}_{t}^{i, r}+v \hat{\bar{c}}_{t}^{i, r}=0 .
\end{aligned}
$$

Aggregate demand. We have

$$
Y_{t}^{i}=(1-\alpha) C_{t}^{i}\left(S_{t}^{i}\right)^{\alpha}+\alpha \frac{C_{t}^{i, o}\left(S_{t}^{i}\right)^{\alpha}}{\Theta_{t}^{i}} \int_{0}^{1} \frac{\Theta_{t}^{j} C_{t}^{j}}{C_{t}^{j, o}} d j+G_{t}^{i} .
$$

Linearizing, we have

$$
\frac{1}{1-v^{2}} y_{t}^{i}=(1-\alpha)\left(\theta_{t}^{i}+c_{t}^{i}+c_{t}^{*, o}-c_{t}^{i, o}\right)+s_{t}^{i}+\alpha c_{t}^{*}+\frac{v}{1-v} g_{t}^{i} .
$$


Therefore, we have

$$
\begin{aligned}
\text { (Natural) } & \frac{1}{1-v} \tilde{y}_{t}^{i}=\tilde{s}_{t}^{i}+(1-\alpha)\left(\tilde{\theta}_{t}^{i}+\tilde{c}_{t}^{i}-\tilde{c}_{t}^{i, o}\right)+\frac{v}{1-v} \tilde{g}_{t}^{i} \\
\text { (Gaps from natural) } & \frac{1}{1-v} \hat{y}_{t}^{i}=\hat{s}_{t}^{i}+\alpha c_{t}^{*}+(1-\alpha)\left(\hat{\theta}_{t}^{i}+\hat{c}_{t}^{i}+c_{t}^{*, o}-\hat{c}_{t}^{i, o}\right)+\frac{v}{1-v} \hat{g}_{t}^{i} \\
\text { (Gaps from union) } & \frac{1}{1-v} \hat{\bar{y}}_{t}^{i}=\hat{\bar{s}}_{t}^{i}+(1-\alpha)\left(\hat{\bar{\theta}}_{t}^{i}+\hat{\bar{c}}_{t}^{i}-\hat{\bar{c}}_{t}^{i, o}\right)+\frac{v}{1-v} \hat{\bar{g}}^{i} .
\end{aligned}
$$

NFA. Log-linearizing (52), we find

$$
N F A_{0}^{i}=-\int_{0}^{\infty} e^{-\rho t}\left(y_{t}^{i}-\alpha(1-v) s_{t}^{i}-(1-v) c_{t}^{i}-v g_{t}^{i}\right) d t,
$$

From (76), we have

$$
\begin{aligned}
\text { (Natural) } & N \tilde{F} A_{0}^{i}=-\int_{0}^{\infty} e^{-\rho t}\left(\tilde{y}_{t}^{i}-\alpha(1-v) \tilde{s}_{t}^{i}-(1-v) \tilde{c}_{t}^{i}-v \tilde{g}_{t}^{i}\right) d t=0 \\
\text { (Gaps from natural) } & N \hat{F} A_{0}^{i}=-\int_{0}^{\infty} e^{-\rho t}\left(\hat{y}_{t}^{i}-\alpha(1-v) \hat{s}_{t}^{i}-(1-v) \hat{c}_{t}^{i}-v \hat{g}_{t}^{i}\right) d t \\
\text { (Gaps from union) } & N \hat{\bar{F}} A_{0}^{i}=-\int_{0}^{\infty} e^{-\rho t}\left(\hat{y}_{t}^{i}-\alpha(1-v) \hat{\bar{s}}_{t}^{i}-(1-v) \hat{\bar{c}}_{t}^{i}-v \hat{\bar{g}}_{t}^{i}\right) d t .
\end{aligned}
$$

Transfer conversion. We have

$$
\tau_{t}^{i, r}=T_{t}^{i, r}-Z_{H, t}^{i}=T_{t}^{i, r}-Y_{t}^{i}\left(S_{t}^{i}\right)^{-\alpha}+\frac{1}{1-v} C_{t}^{i, o}\left(N_{t}^{i, o}\right)^{\phi} N_{t}^{i} .
$$

Linearizing, we have

$$
\tau_{t}^{i, r}=t_{t}^{i, r}-\left(y_{t}^{i}-\alpha s_{t}^{i}-c_{t}^{i, o}-\phi n_{t}^{i, o}-n_{t}^{i}\right)
$$

Therefore, we have

(Natural)

(Gaps from natural)

(Gaps from union)

$$
\begin{aligned}
& \tilde{\tau}_{t}^{i, r}=\tilde{t}_{t}^{i, r}-\left(\tilde{y}_{t}^{i}-\alpha \tilde{s}_{t}^{i}-\tilde{c}_{t}^{i, o}-\phi \tilde{n}_{t}^{i, o}-\tilde{n}_{t}^{i}\right) \\
& \hat{\tau}_{t}^{i, r}=\hat{t}_{t}^{i, r}-\left(\hat{y}_{t}^{i}-\alpha \hat{s}_{t}^{i}-\hat{c}_{t}^{i, o}-\phi \hat{n}_{t}^{i, o}-\hat{n}_{t}^{i}\right) \\
& \hat{\bar{\tau}}_{t}^{i, r}=\hat{\bar{t}}_{t}^{i, r}-\left(\hat{\bar{y}}_{t}^{i}-\alpha \hat{\bar{s}}_{t}^{i}-\hat{\bar{c}}_{t}^{i, o}-\phi \hat{n}_{t}^{i, o}-\hat{n}_{t}^{i}\right) .
\end{aligned}
$$

Similar conditions hold for $\tau_{t}^{i, o}$.

Government budget constraint. We have

$$
0=P_{H, t}^{i} G_{t}^{i}+\chi P_{t}^{i} \tau_{t}^{i, r}+(1-\chi) P_{t}^{i} \tau_{t}^{i, o},
$$

which we can linearize as

$$
\alpha v s_{t}^{i}=\chi \tau_{t}^{i, r}+(1-\chi) \tau_{t}^{i, o}+v g_{t}^{i}
$$


Therefore, we have

(Natural)

(Gaps from natural)

(Gaps from union)

$$
\begin{aligned}
& \alpha v \tilde{s}_{t}^{i}=\chi \tilde{\tau}_{t}^{i, r}+(1-\chi) \tilde{\tau}_{t}^{i, o}+v \tilde{g}_{t}^{i} \\
& \alpha v \hat{s}_{t}^{i}=\chi \hat{\tau}_{t}^{i, r}+(1-\chi) \hat{\tau}_{t}^{i, o}+v \hat{g}_{t}^{i} \\
& \alpha v \hat{\bar{s}}_{t}^{i}=\chi \hat{\bar{\tau}}_{t}^{i, r}+(1-\chi) \hat{\bar{\tau}}_{t}^{i, o}+v \hat{\bar{g}}_{t}^{i} .
\end{aligned}
$$

Symmetric fiscal union transfers. We want to rebate the transfers received by optimizers from the union to HtM agents so that each agent receives the same amount. This condition is given by

$$
N F A_{0}^{i}=\int_{0}^{\infty} e^{-\rho t}\left[\tau_{t}^{i, r}+\left(S_{t}^{i}\right)^{-\alpha} G_{t}^{i}\right] d t .
$$

Linearizing yields

$$
N F A_{0}^{i}=\int_{0}^{\infty} e^{-\rho t}\left[\tau_{t}^{i, r}+v g_{t}^{i}-v \alpha s_{t}^{i}\right] d t
$$

This implies

$$
\begin{aligned}
\text { (Natural) } & N \tilde{F} A_{0}^{i}=\int_{0}^{\infty} e^{-\rho t}\left[\tilde{\tau}_{t}^{i, r}+v \tilde{g}_{t}^{i}-v \alpha \tilde{s}_{t}^{i}\right] d t=0 \\
\text { (Gaps from natural) } & N \hat{F} A_{0}^{i}=\int_{0}^{\infty} e^{-\rho t}\left[\hat{\tau}_{t}^{i, r}+v \hat{g}_{t}^{i}-v \alpha \hat{s}_{t}^{i}\right] d t=0 \\
\text { (Gaps from union) } & N \hat{\bar{F}} A_{0}^{i}=\int_{0}^{\infty} e^{-\rho t}\left[\hat{\bar{\tau}}_{t}^{i, r}+v \hat{\bar{g}}_{t}^{i}-v \alpha \hat{\bar{s}}_{t}^{i}\right] d t=0 .
\end{aligned}
$$

Supply side with flexible prices. Under the natural, the supply side simplifies to the single equation

$$
\tilde{y}_{t}^{i}=\tilde{c}_{t}^{i}+\alpha \tilde{s}_{t}^{i}+(1+\phi) \tilde{n}_{t}^{i}
$$

Supply side with sticky prices. Let $\lambda=\rho_{\delta}\left(\rho+\rho_{\delta}\right)$, where $\rho_{\delta}$ is the arrival rate of price changes. Using the supply-side equilibrium conditions in continuous time, we can write the Phillips Curve as

(Gaps from natural)

(Gaps from union)

$$
\begin{aligned}
& \dot{\hat{\pi}}_{H, t}^{i}=\rho \hat{\pi}_{H, t}^{i}-\lambda\left(\phi \hat{n}_{t}^{i}+\hat{c}_{t}^{i}+\alpha \hat{\bar{s}}_{t}^{i}\right) \\
& \dot{\bar{\pi}}_{H, t}^{i}=\rho \hat{\bar{\pi}}_{H, t}^{i}-\lambda\left(\phi \hat{\bar{n}}_{t}^{i}+\hat{\bar{c}}_{t}^{i}+\alpha \hat{\bar{s}}_{t}^{i}\right) .
\end{aligned}
$$

\section{D.2 Allocations Given Exogenous Policies}

We begin this section by characterizing and solving for the natural allocation. We then summarize the allocation with policy in gaps from the natural, which will be our exclusive focus for all subsequent analysis. Furthermore, we derive the (IS) and (NKPC) equations, an initial condition with which we can solve the dynamical system later on, as well as country $i$ 's generalized budget constraint. Finally, we solve analytically the allocation under sticky prices but without policy, which we 
will later use for several of our numerical exercises.

\section{D.2.1 Natural Allocation}

We define the natural as the allocation with flexible prices and with passive policy. As discussed in the main text, we allow government spending and taxes to move with the technology shock to ensure that the flexible price allocation is efficient. At the natural allocation, taxes are uniform across agents and the government budget is balanced in every period. This implies $\tilde{g}_{t}^{i}=a_{t}^{i}$ and $\tilde{\theta}_{t}^{i}=\tilde{\theta}^{i}$ for all $i$. Furthermore, there are no fiscal union transfers so that $N \tilde{F} A_{0}^{i}=0$. This implies that $\tilde{\tau}_{t}^{i, r}$ and $\tilde{\tau}_{t}^{i, o}$ merely denote tax rebates that are equally imposed on both agents to balance the per-period government budget constraint. We note here again that under idiosyncratic shocks to any measure-0 country $i$, the union-wide natural allocation remains unaffected and $\tilde{x}_{t}^{*}=0$ for all variables $\tilde{x}_{t}$.

The natural allocation is given by the system of equations

$$
\begin{aligned}
\tilde{y}_{t}^{i} & =a_{t}^{i}+\tilde{n}_{t}^{i} \\
\tilde{c}_{t}^{i} & =\chi \tilde{c}_{t}^{i, r}+(1-\chi) \tilde{c}_{t}^{i, o} \\
\tilde{n}_{t}^{i} & =\chi \tilde{n}_{t}^{i, r}+(1-\chi) \tilde{n}_{t}^{i, o} \\
\tilde{c}_{t}^{i, o}+\phi \tilde{n}_{t}^{i, o} & =\tilde{c}_{t}^{i, r}+\phi \tilde{n}_{t}^{i, r} \\
\tilde{c}_{t}^{i, o} & =\tilde{\theta}_{t}^{i}+(1-\alpha) \tilde{s}_{t}^{i} \\
0 & =\tilde{t}_{t}^{i, r}+(1+\phi) \tilde{n}_{t}^{i, r}+v \tilde{c}_{t}^{i, r} \\
\frac{1}{1-v} \tilde{y}_{t}^{i} & =\tilde{s}_{t}^{i}+(1-\alpha)\left(\tilde{\theta}_{t}^{i}+\tilde{c}_{t}^{i}-\tilde{c}_{t}^{i, o}\right)+\frac{v}{1-v} \tilde{g}_{t}^{i} \\
\tilde{y}_{t}^{i} & =\tilde{c}_{t}^{i}+\alpha \tilde{s}_{t}^{i}+(1+\phi) \tilde{n}_{t}^{i}
\end{aligned}
$$

as well as the following conditions for transfers and the NFA

$$
\begin{aligned}
\alpha v \tilde{s}_{t}^{i} & =\chi \tilde{\tau}_{t}^{i, r}+(1-\chi) \tilde{\tau}_{t}^{i, o}+v \tilde{g}_{t}^{i} \\
\tilde{\tau}_{t}^{i, o} & =\tilde{t}_{t}^{i, o}+\frac{\chi}{1-\chi}\left(\tilde{y}_{t}^{i}-\alpha \tilde{s}_{t}^{i}-\tilde{c}_{t}^{i, o}-\phi \tilde{n}_{t}^{i, o}-\tilde{n}_{t}^{i}\right) \\
\tilde{\tau}_{t}^{i, r} & =\tilde{t}_{t}^{i, r}-\left(\tilde{y}_{t}^{i}-\alpha \tilde{s}_{t}^{i}-\tilde{c}_{t}^{i, o}-\phi \tilde{n}_{t}^{i, o}-\tilde{n}_{t}^{i}\right) \\
0 & =-\int_{0}^{\infty} e^{-\rho t}\left(\tilde{y}_{t}^{i}-\alpha(1-v) \tilde{s}_{t}^{i}-(1-v) \tilde{c}_{t}^{i}-v \tilde{g}_{t}^{i}\right) d t \\
0 & =\int_{0}^{\infty} e^{-\rho t}\left(\tilde{\tau}_{t}^{i, r}+v \tilde{g}_{t}^{i}-\alpha v \tilde{s}_{t}^{i}\right) d t=0 .
\end{aligned}
$$

We highlight two important features of the natural allocation: First, for any level of steady state government spending $v \in[0,1]$, the natural allocation is $\chi$-invariant and $\tilde{\theta}^{i}=0$. Second, for any level of steady state government spending $v \in[0,1]$ and for any share of hand-to-mouth agents $\chi \in[0,1]$, the natural allocation is symmetric, with $\tilde{n}_{t}^{i, r}=\tilde{n}_{t}^{i, o}$ and $\tilde{c}_{t}^{i, r}=\tilde{c}_{t}^{i, o}$ for all $t$. 
We will now solve the natural allocation in closed form. We use $\tilde{g}_{t}^{i}=a_{t}^{i}$. Solving out for $\tilde{\theta}_{t}^{i}$ $\tilde{n}_{t}^{i}=\tilde{y}_{t}^{i}-a_{t}^{i}, \tilde{c}_{t}^{i}=\chi \tilde{c}_{t}^{i, r}+(1-\chi) \tilde{c}_{t}^{i, o}$, and $\tilde{c}_{t}^{i, o}=\tilde{\theta}_{t}^{i}+(1-\alpha) \tilde{s}_{t}^{i}$, we find

$$
\begin{aligned}
\tilde{y}_{t}^{i}-a_{t}^{i} & =\chi \tilde{n}_{t}^{i, r}+(1-\chi) \tilde{n}_{t}^{i, o} \\
\phi \tilde{n}_{t}^{i, o}+(1-\alpha) \tilde{s}_{t}^{i} & =\tilde{c}_{t}^{i, r}+\phi \tilde{n}_{t}^{i, r} \\
\alpha v \tilde{s}_{t}^{i}+(1+\phi) \tilde{n}_{t}^{i, r}+v \tilde{c}_{t}^{i, r} & =\tilde{s}_{t}^{i}+\phi \tilde{n}_{t}^{i, o}-(1-v) a_{t}^{i} \\
\frac{1}{1-v} \tilde{y}_{t}^{i} & =\left[1-\chi(1-\alpha)^{2}\right] \tilde{s}_{t}^{i}+\chi(1-\alpha) \tilde{c}_{t}^{i, r}+\frac{v}{1-v} a_{t}^{i} \\
(1+\phi) a_{t}^{i} & =\chi \tilde{c}_{t}^{i, r}+[(1-\chi)(1-\alpha)+\alpha] \tilde{s}_{t}^{i}+\phi \tilde{y}_{t}^{i} .
\end{aligned}
$$

From here, we can solve for $\tilde{c}_{t}^{i, r}$ and $\tilde{n}_{t}^{i, r}$, yielding

$$
\begin{aligned}
\tilde{c}_{t}^{i, r} & =\frac{\phi}{1-\chi+(1-v) \phi} \tilde{y}_{t}^{i}-\frac{v \phi}{1-\chi+(1-v) \phi} a_{t}^{i}+\frac{(1-\alpha)(1-\chi)-(1-v) \alpha \phi}{1-\chi+(1-v) \phi} \tilde{s}_{t}^{i} \\
\tilde{n}_{t}^{i, r} & =\frac{(1-v) \phi}{1-\chi+(1-v) \phi} \tilde{y}_{t}^{i}-\frac{(1-v)(1-\chi+\phi)}{1-\chi+(1-v) \phi} a_{t}^{i}+\frac{(1-\chi)(1-v)}{1-\chi+(1-v) \phi} \tilde{s}_{t}^{i} .
\end{aligned}
$$

Plugging these into the aggregate demand relation, we can solve for the terms of trade and find that $\tilde{s}_{t}^{i}=\frac{1}{1-v} \tilde{y}_{t}^{i}-\frac{v}{1-v} a_{t}^{i}$. Using these to solve for the output gap, finally, we find

$$
\tilde{y}_{t}^{i}=a_{t}^{i}
$$

and similarly

$$
\tilde{s}_{t}^{i}=a_{t}^{i}
$$

Solving the remainder of the allocation is straightforward using these two results. Finally, we note here that

$$
\begin{aligned}
\dot{\tilde{s}}_{t}^{i} & =-\dot{p}_{H, t}^{i} \\
& =-\psi e^{-\psi t} a_{0}^{i} .
\end{aligned}
$$

We will make use of this result later on.

\section{D.2.2 Allocation with Policy}

We now present the allocation in terms of gaps from the natural. Recall that $\hat{\bar{x}}_{t}^{i}=\ln \left(X_{t}^{i}\right)-\ln \left(\tilde{X}_{t}^{i}\right)$ for all variables $X_{t}^{i}$, where $\tilde{X}_{t}^{i}$ denotes the natural allocation.

The linear model with policy is given by

$$
\begin{aligned}
\hat{\bar{c}}_{t}^{i, o}+\phi \hat{\bar{n}}_{t}^{i, o} & =\hat{\bar{c}}_{t}^{i, r}+\phi \hat{\bar{n}}_{t}^{i, r} \\
\hat{\bar{c}}_{t}^{i, o} & =\hat{\bar{\theta}}_{t}^{i}+(1-\alpha) \hat{\bar{s}}_{t}^{i} \\
\hat{\bar{c}}_{t}^{i} & =\chi \hat{\bar{c}}_{t}^{i, r}+(1-\chi) \hat{\bar{c}}_{t}^{i, o}
\end{aligned}
$$




$$
\begin{aligned}
\hat{\bar{n}}_{t}^{i} & =\chi \hat{\bar{n}}_{t}^{i, r}+(1-\chi) \hat{\bar{n}}_{t}^{i, o} \\
\hat{\bar{y}}_{t}^{i} & =\hat{\bar{n}}_{t}^{i} \\
\hat{\bar{t}}_{t}^{i, r} & =-(1+\phi) \hat{\bar{n}}_{t}^{i, r}-v \hat{\bar{c}}_{t}^{i, r} \\
\frac{1}{1-v} \hat{\bar{y}}_{t}^{i} & =\hat{\bar{s}}_{t}^{i}+(1-\alpha)\left(\hat{\bar{\theta}}_{t}^{i}+\hat{\bar{c}}_{t}^{i}-\hat{\bar{c}}_{t}^{i, o}\right)+\frac{v}{1-v} \hat{\bar{g}}_{t}^{i} \\
\hat{\bar{\tau}}_{t}^{i, r} & =\hat{\bar{t}}_{t}^{i, r}-\left(\hat{\bar{y}}_{t}^{i}-\alpha \hat{\bar{s}}_{t}^{i}-\hat{\bar{c}}_{t}^{i, o}-\phi \hat{\bar{n}}_{t}^{i, o}-\hat{\bar{n}}_{t}^{i}\right) \\
\alpha v \hat{\bar{s}}_{t}^{i} & =\chi \hat{\bar{\tau}}_{t}^{i, r}+(1-\chi) \hat{\bar{\tau}}_{t}^{i, o}+v \hat{\bar{g}}_{t}^{i} \\
N \hat{\bar{F}}_{A_{0}}^{i} & =\int_{0}^{\infty} e^{-\rho t}\left[\hat{\bar{\tau}}_{t}^{i, r}+v \hat{\bar{g}}_{t}^{i}-v \alpha \hat{\bar{s}}_{t}^{i}\right] d t \\
N \hat{\bar{F}}_{0}^{i} & =-\int_{0}^{\infty} e^{-\rho t}\left[\hat{\bar{y}}_{t}^{i}-\alpha(1-v) \hat{\bar{s}}_{t}^{i}-(1-v) \hat{\bar{c}}_{t}^{i}-v \hat{\bar{g}}_{t}^{i}\right] d t \\
\dot{\bar{\tau}}_{H, t}^{i} & =\rho \hat{\bar{\pi}}_{H, t}^{i}-\lambda\left(\phi \hat{\bar{n}}_{t}^{i}+\hat{\bar{c}}_{t}^{i}+\alpha \hat{\bar{s}}_{t}^{i}\right) \\
\pi_{t}^{i} & =\pi_{H, t}^{i}+\alpha \dot{s}_{t}^{i} \\
\dot{\bar{c}}_{t}^{i, o} & =i_{t}^{i}-\pi_{t}^{i}-\rho .
\end{aligned}
$$

Note that for all variables we have $\int_{0}^{1} \tilde{x}_{t}^{i} d i=0$, so that $\tilde{x}_{t}^{i}=\tilde{x}_{t}^{i}$. We used this simplification above.

We furthermore need an initial value condition to close the system and pin down the solution of this first-order linear system of ODEs. We will derive the initial condition below.

Before doing so, it will be convenient to solve this system in terms of $\hat{y}_{t}^{i}, \hat{\bar{\tau}}_{t}^{i, r}, \hat{\theta}_{t}^{i}$ and $\hat{\bar{g}}_{t}^{i}$. We start by solving out for $\hat{\bar{n}}_{t}^{i}, \hat{\bar{c}}_{t}^{i}$, and $\hat{\bar{c}}_{t}^{i, 0}$. This yields

$$
\begin{aligned}
\hat{\bar{\theta}}_{t}^{i}+(1-\alpha) \hat{\bar{s}}_{t}^{i}+\phi \hat{\bar{n}}_{t}^{i, o} & =\hat{\bar{c}}_{t}^{i, r}+\phi \hat{\bar{n}}_{t}^{i, r} \\
\hat{\bar{y}}_{t}^{i} & =\chi \hat{\bar{n}}_{t}^{i, r}+(1-\chi) \hat{\bar{n}}_{t}^{i, o} \\
\hat{\bar{t}}_{t}^{i, r} & =-(1+\phi) \hat{\bar{n}}_{t}^{i, r}-v \hat{\bar{c}}_{t}^{i, r} \\
\frac{1}{1-v} \hat{\bar{y}}_{t}^{i} & =(1-\alpha)(1-\chi) \hat{\bar{\theta}}_{t}^{i}+\chi(1-\alpha) \hat{\bar{c}}_{t}^{i, r}+\left[1-\chi(1-\alpha)^{2}\right] \hat{\bar{s}}_{t}^{i}+\frac{v}{1-v} \hat{\bar{g}}_{t}^{i} \\
\hat{\bar{t}}_{t}^{i, r} & =\hat{\bar{\tau}}_{t}^{i, r}+\hat{\bar{y}}_{t}^{i}-\alpha \hat{\bar{s}}_{t}^{i}-\hat{\bar{c}}_{t}^{i, o}-\phi \hat{\bar{n}}_{t}^{i, o}-\hat{\bar{n}}_{t}^{i} .
\end{aligned}
$$

Next, we solve out for $\hat{t}_{t}^{i, r}$ using the last equation in the previous block, and for $\hat{\bar{n}}_{t}^{i, o}=\frac{1}{1-\chi} \hat{\bar{y}}_{t}^{i}-$ $\frac{\chi}{1-\chi} \hat{n}_{t}^{i, r}$. We are interested in the following two equations,

$$
\begin{aligned}
\hat{\bar{\theta}}_{t}^{i}+(1-\alpha) \hat{\bar{s}}_{t}^{i}+\frac{\phi}{1-\chi} \hat{\bar{y}}_{t}^{i} & =\hat{\bar{c}}_{t}^{i, r}+\frac{\phi}{1-\chi} \hat{\bar{n}}_{t}^{i, r} \\
\hat{\bar{\tau}}_{t}^{i, r}-\hat{\bar{s}}_{t}^{i}-\hat{\bar{\theta}}_{t}^{i}-\frac{\phi}{1-\chi} \hat{\bar{y}}_{t}^{i}+\left(1+\phi+\frac{\chi \phi}{1-\chi}\right) \hat{\bar{n}}_{t}^{i, r} & =-v \hat{\bar{c}}_{t}^{i, r},
\end{aligned}
$$

which we can now use to solve for $\hat{\bar{c}}_{t}^{i, r}$ and $\hat{\hat{n}}_{t}^{i, r}$. In particular, this yields

$$
\hat{\bar{n}}_{t}^{i, r}=\frac{1-\chi}{1-\chi+(1-v) \phi}\left((1-v) \frac{\phi}{1-\chi} \hat{\bar{y}}_{t}^{i}+(1-v) \hat{\theta}_{t}^{i}+[1-v(1-\alpha)] \hat{s}_{t}^{i}-\hat{\bar{\tau}}_{t}^{i, r}\right)
$$


and

$$
\hat{\bar{c}}_{t}^{i, r}=\frac{1}{1-\chi+(1-v) \phi}\left((1-\chi) \hat{\bar{\theta}}_{t}^{i}+\phi \hat{\bar{y}}_{t}^{i}+[(1-\alpha)(1-\chi)-\alpha \phi] \hat{\bar{s}}_{t}^{i}+\phi \hat{\bar{\tau}}_{t}^{i, r}\right) .
$$

Finally, we use the linearized aggregate demand relation to solve for $\hat{\bar{s}}_{t}^{i}$. We find

$$
\begin{aligned}
\hat{\bar{s}}_{t}^{i}= & \left(1-(1-\alpha) \chi \frac{(1-v) \phi+\alpha v \phi}{1-\chi+(1-v) \phi}\right)^{-1}\left[\left(\frac{1}{1-v}-\frac{(1-\alpha) \chi \phi}{1-\chi+(1-v) \phi}\right) \hat{\bar{y}}_{t}^{i}-\frac{v}{1-v} \hat{\bar{g}}_{t}^{i}\right. \\
& \left.-(1-\alpha)(1-\chi) \frac{1+\phi-v \phi}{1-\chi+(1-v) \phi} \hat{\bar{\theta}}_{t}^{i}-\frac{(1-\alpha) \chi \phi}{1-\chi+(1-v) \phi} \hat{\bar{\tau}}_{t}^{i, r}\right] \\
\equiv & v_{y} \hat{\bar{y}}_{t}^{i}+v_{g} \hat{\bar{g}}_{t}^{i}+v_{\theta} \hat{\bar{\theta}}_{t}^{i}+v_{\tau} \hat{\bar{\tau}}_{t}^{i, r} .
\end{aligned}
$$

Therefore, we can for the future introduce the following notation

$$
\begin{aligned}
\hat{\bar{c}}_{t}^{i, r} & =\psi_{y} \hat{\bar{y}}_{t}^{i}+\psi_{\theta} \hat{\bar{\theta}}_{t}^{i}+\psi_{\tau} \hat{\bar{\tau}}_{t}^{i, r}+\psi_{g} \hat{\bar{g}}_{t}^{i} \\
\hat{\bar{n}}_{t}^{i, r} & =\eta_{y} \hat{\bar{y}}_{t}^{i}+\eta_{g} \hat{\bar{g}}_{t}^{i}+\eta_{\theta} \hat{\bar{\theta}}_{t}^{i}+\eta_{\tau} \hat{\bar{\tau}}_{t}^{i, r} \\
\hat{\bar{c}}_{t}^{i} & =\zeta_{y} \hat{\bar{y}}_{t}^{i}+\zeta_{\theta} \hat{\bar{\theta}}_{t}^{i}+\zeta_{\tau} \hat{\bar{\tau}}_{t}^{i, r}+\zeta_{g} \hat{\bar{g}}_{t}^{i}
\end{aligned}
$$

where

$$
\begin{aligned}
& \psi_{y}=\frac{\phi}{1-\chi+(1-v) \phi}+\left[1-\alpha-\frac{(1-v) \phi+\alpha v \phi}{1-\chi+(1-v) \phi}\right] v_{y} \\
& \psi_{\theta}=\frac{1-\chi}{1-\chi+(1-v) \phi}+\left[1-\alpha-\frac{(1-v) \phi+\alpha v \phi}{1-\chi+(1-v) \phi}\right] v_{\theta} \\
& \psi_{\tau}=\frac{\phi}{1-\chi+(1-v) \phi}+\left[1-\alpha-\frac{(1-v) \phi+\alpha v \phi}{1-\chi+(1-v) \phi}\right] v_{\tau} \\
& \psi_{g}=\left[1-\alpha-\frac{(1-v) \phi+\alpha v \phi}{1-\chi+(1-v) \phi}\right] v_{g}
\end{aligned}
$$

and

$$
\begin{aligned}
\eta_{y} & =\frac{1-\chi}{1-\chi+(1-v) \phi}\left[(1-v) \frac{\phi}{1-\chi}+[1-v(1-\alpha)] v_{y}\right] \\
\eta_{g} & =\frac{1-\chi}{1-\chi+(1-v) \phi}[1-v(1-\alpha)] v_{g} \\
\eta_{\theta} & =\frac{1-\chi}{1-\chi+(1-v) \phi}\left[1-v+[1-v(1-\alpha)] v_{\theta}\right] \\
\eta_{\tau} & =\frac{1-\chi}{1-\chi+(1-v) \phi}\left[-1+[1-v(1-\alpha)] v_{\tau}\right],
\end{aligned}
$$

and

$$
\zeta_{y}=\chi \psi_{y}+(1-\chi)(1-\alpha) v_{y}
$$




$$
\begin{aligned}
& \zeta_{g}=\chi \psi_{g}+(1-\chi)(1-\alpha) v_{g} \\
& \zeta_{\tau}=\chi \psi_{\tau}+(1-\chi)(1-\alpha) v_{\tau} \\
& \zeta_{\theta}=\chi \psi_{\theta}+(1-\chi)\left[1+(1-\alpha) v_{\theta}\right]
\end{aligned}
$$

\section{D.2.3 IS equation}

We start from the linearized aggregate demand equation and use slightly more convenient notation to write

$$
\Lambda_{y} \hat{\bar{y}}_{t}^{i}=\Lambda_{s} \hat{\bar{s}}_{t}^{i}+\Lambda_{g} \hat{\bar{g}}_{t}^{i}+\Lambda_{\theta} \hat{\bar{\theta}}_{t}^{i}+\Lambda_{\tau} \hat{\bar{\tau}}_{t}^{i, r}
$$

where

$$
\begin{aligned}
& \Lambda_{y}=\frac{1}{1-v}-\frac{(1-\alpha) \chi \phi}{1-\chi+(1-v) \phi} \\
& \Lambda_{s}=1-(1-\alpha) \chi \frac{(1-v) \phi+\alpha v \phi}{1-\chi+(1-v) \phi} \\
& \Lambda_{\theta}=(1-\alpha)(1-\chi) \frac{1+\phi-v \phi}{1-\chi+(1-v) \phi} \\
& \Lambda_{g}=\frac{v}{1-v} \\
& \Lambda_{\tau}=\frac{(1-\alpha) \chi \phi}{1-\chi+(1-v) \phi^{\prime}}
\end{aligned}
$$

and where we have the easy conversions: $v_{y}=\frac{\Lambda_{y}}{\Lambda_{s}}, v_{g}=-\frac{\Lambda_{g}}{\Lambda_{s}}, v_{\theta}=-\frac{\Lambda_{\theta}}{\Lambda_{s}}$, and $v_{\tau}=-\frac{\Lambda_{\tau}}{\Lambda_{s}}$. Differentiating, we have

$$
\Lambda_{y} \dot{\hat{\bar{y}}}_{t}^{i}=\Lambda_{s} \dot{\hat{\overline{\hat{S}}}}_{t}^{i}+\Lambda_{g} \dot{\hat{\bar{g}}}_{t}^{i}+\Lambda_{\theta} \dot{\overline{\hat{\theta}}}_{t}^{i}+\Lambda_{\tau} \dot{\overline{\hat{\tau}}}_{t}^{i, r},
$$

To derive the (IS) equation, all that is left to do is to find an expression for $\dot{\hat{s}}_{t}^{i}$. From the definition of the terms of trade, we can directly write

$$
\dot{\hat{\bar{s}}}_{t}^{i}=-\left(\hat{\bar{\pi}}_{H, t}^{i}+\dot{\tilde{s}}_{t}^{i}\right)
$$

Therefore, the fully general (IS) equation is given by

$$
\Lambda_{y} \dot{\hat{\bar{y}}}_{t}^{i}=-\Lambda_{s}\left(\hat{\bar{\pi}}_{H, t}^{i}+\dot{\tilde{s}}_{t}^{i}\right)+\Lambda_{g} \dot{\hat{\bar{g}}}_{t}^{i}+\Lambda_{\theta} \dot{\hat{\bar{\theta}}}_{t}^{i}+\Lambda_{\tau} \dot{\hat{\bar{\tau}}}_{t}^{i, r} .
$$

\section{D.2.4 NKPC}

We start with the general Phillips Curve

$$
\dot{\pi}_{H, t}^{i}=\rho \pi_{H, t}^{i}-\lambda\left(\phi n_{t}^{i}+c_{t}^{i}+\alpha s_{t}^{i}-a_{t}^{i}\right)
$$


Noting that

$$
\phi \tilde{n}_{t}^{i}+\tilde{c}_{t}^{i}+\alpha \tilde{s}_{t}^{i}-a_{t}^{i}=0
$$

under the natural allocation, and taking deviations from union aggregates, we can write the NKPC in gap notation as

$$
\dot{\overline{\bar{\pi}}}_{H, t}^{i}=\rho \hat{\bar{\pi}}_{H, t}^{i}-\lambda\left(\phi \hat{\bar{n}}_{t}^{i}+\hat{\bar{c}}_{t}^{i}+\alpha \hat{\bar{s}}_{t}^{i}\right),
$$

Using expressions we derived earlier, we have

$$
\dot{\overline{\hat{\tau}}}_{H, t}^{i}=\rho \hat{\bar{\pi}}_{H, t}^{i}-\lambda \phi \hat{\bar{y}}_{t}^{i}-\lambda\left(\zeta_{y} \hat{\bar{y}}_{t}^{i}+\zeta_{\theta} \hat{\bar{\theta}}_{t}^{i}+\zeta_{\tau} \hat{\bar{\tau}}_{t}^{i, r}+\zeta_{g} \hat{\bar{g}}_{t}^{i}\right)-\lambda \alpha\left(v_{y} \hat{\bar{y}}_{t}^{i}+v_{g} \hat{\bar{g}}_{t}^{i}+v_{\theta} \hat{\bar{\theta}}_{t}^{i}+v_{\tau} \hat{\bar{\tau}}_{t}^{i, r}\right),
$$

or, collecting terms,

$$
\dot{\overline{\bar{\pi}}}_{H, t}^{i}=\rho \hat{\bar{\pi}}_{H, t}^{i}-\kappa_{y} \hat{\bar{y}}_{t}^{i}-\kappa_{\theta} \hat{\bar{\theta}}_{t}^{i}-\kappa_{\tau} \hat{\bar{\tau}}_{t}^{i, r}-\kappa_{g} \hat{\bar{g}}_{t}^{i}
$$

where

$$
\begin{aligned}
& \kappa_{y}=\lambda\left(\phi+\zeta_{y}+\alpha v_{y}\right) \\
& \kappa_{g}=\lambda\left(\zeta_{g}+\alpha v_{g}\right) \\
& \kappa_{\tau}=\lambda\left(\zeta_{\tau}+\alpha v_{\tau}\right) \\
& \kappa_{\theta}=\lambda\left(\zeta_{\theta}+\alpha v_{\theta}\right) .
\end{aligned}
$$

\section{D.2.5 Initial Condition}

As for the (IS) equation, we start with the linearized aggregate demand relation. We have

$$
\Lambda_{y} \hat{\bar{y}}_{0}^{i}=\Lambda_{s} \hat{\bar{s}}_{0}^{i}+\Lambda_{g} \hat{\bar{g}}_{0}^{i}+\Lambda_{\theta} \hat{\bar{\theta}}_{0}^{i}+\Lambda_{\tau} \hat{\bar{\tau}}_{0}^{i, r}
$$

We can obtain an initial condition for our dynamic system by noting that

$$
s_{0}^{i}=\hat{\bar{s}}_{t}^{i}+\tilde{s}_{0}^{i}=0,
$$

implying that $\hat{\bar{s}}_{t}^{i}=-\tilde{s}_{0}^{i}$. Using this, we can write

$$
\Lambda_{y} \hat{\bar{y}}_{0}^{i}=-\Lambda_{s} \tilde{s}_{0}^{i}+\Lambda_{g} \hat{\bar{g}}_{0}^{i}+\Lambda_{\theta} \hat{\bar{\theta}}_{0}^{i}+\Lambda_{\tau} \hat{\bar{\tau}}_{0}^{i, r}
$$

\section{D.2.6 Country- $i$ Budget Constraint}

Finally, it remains to simplify the net foreign asset relations. Solving out for $N \hat{\bar{F}} A_{0}^{i}$, we can write

$$
\int_{0}^{\infty} e^{-\rho t}\left[\hat{\bar{\tau}}_{t}^{i, r}+v \hat{\bar{g}}_{t}^{i}-v \alpha \hat{\bar{s}}_{t}^{i}\right] d t=-\int_{0}^{\infty} e^{-\rho t}\left[\hat{\bar{y}}_{t}^{i}-\alpha(1-v) \hat{\bar{s}}_{t}^{i}-(1-v) \hat{\bar{c}}_{t}^{i}-v \hat{\bar{g}}_{t}^{i}\right] d t .
$$


We can simplify to get

$$
\begin{gathered}
0=\int_{0}^{\infty} e^{-\rho t}\left[\hat{\bar{y}}_{t}^{i}+\hat{\tau}_{t}^{i, r}-(1-v) \chi\left(\psi_{y} \hat{\bar{y}}_{t}^{i}+\psi_{\theta} \hat{\theta}_{t}^{i}+\psi_{\tau} \hat{\bar{\tau}}_{t}^{i, r}+\psi_{g} \hat{\bar{g}}_{t}^{i}\right)-(1-v)(1-\chi) \hat{\bar{\theta}}_{t}^{i}\right. \\
\left.-[\alpha+(1-v)(1-\chi)(1-\alpha)]\left(v_{y} \hat{\bar{y}}_{t}^{i}+v_{g} \hat{\bar{g}}_{t}^{i}+v_{\theta} \hat{\theta}_{t}^{i}+v_{\tau} \hat{\bar{\tau}}_{t}^{i, r}\right)\right] d t .
\end{gathered}
$$

Collecting terms, we can write

$$
0=\int_{0}^{\infty} e^{-\rho t}\left[\Gamma_{y} \hat{\hat{y}}_{t}^{i}+\Gamma_{\tau} \hat{\bar{\tau}}_{t}^{i, r}+\Gamma_{\theta} \hat{\theta}_{t}^{i}+\Gamma_{g} \hat{\bar{g}}_{t}^{i}\right] d t
$$

where

$$
\begin{aligned}
& \Gamma_{y}=1-(1-v) \chi \psi_{y}-[\alpha+(1-v)(1-\chi)(1-\alpha)] v_{y} \\
& \Gamma_{\tau}=1-(1-v) \chi \psi_{\tau}-[\alpha+(1-v)(1-\chi)(1-\alpha)] v_{\tau} \\
& \Gamma_{\theta}=-(1-v)(1-\chi)-(1-v) \chi \psi_{\theta}-[\alpha+(1-v)(1-\chi)(1-\alpha)] v_{\theta} \\
& \Gamma_{g}=-(1-v) \chi \psi_{g}-[\alpha+(1-v)(1-\chi)(1-\alpha)] v_{g} .
\end{aligned}
$$

\section{D.2.7 Allocation with Sticky Prices}

Finally, we will solve the allocation presented above under sticky prices but without optimal policy intervention. In particular, we have $N \hat{\bar{F}} A_{0}^{i}=0$ since there are no international transfers. This implies that $\hat{\tau}_{t}^{i, r}=\hat{\bar{\tau}}_{t}^{i, o}=\alpha v \hat{\bar{s}}_{t}^{i}$.

Furthermore, we set $\hat{g}_{t}^{i}=0$ and $\hat{\theta}_{t}^{i}=\hat{\theta}^{i}$ in the absence of government spending policy and capital controls. Since we also have $\hat{\bar{s}}_{t}^{i}=v_{y} \hat{\bar{y}}_{t}^{i}+v_{\tau} \hat{\bar{\tau}}_{t}^{i, r}+v_{\theta} \hat{\bar{\theta}}^{i}$, we can write

$$
\begin{aligned}
& \hat{\bar{\tau}}_{t}^{i, r}=\frac{\alpha v}{1-\alpha v v_{\tau}}\left(v_{y} \hat{\bar{y}}_{t}^{i}+v_{\theta} \hat{\bar{\theta}}^{i}\right) \\
& \dot{\hat{\bar{\tau}}}_{t}^{i, r}=\frac{\alpha v}{1-\alpha v v_{\tau}} v_{y} \dot{\hat{y}}_{t}^{i} .
\end{aligned}
$$

This allocation will be important for our numerical exercises where we compare optimal policy to this no-policy benchmark.

NFA. The general country $i$ budget constraint simplifies to

$$
\begin{aligned}
& 0=\int_{0}^{\infty} e^{-\rho t}\left[\Gamma_{y} \hat{\bar{y}}_{t}^{i}+\Gamma_{\tau} \hat{\bar{\tau}}_{t}^{i, r}+\Gamma_{\theta} \hat{\bar{\theta}}^{i}\right] d t \\
& 0=\int_{0}^{\infty} e^{-\rho t}\left[\left(\Gamma_{y}+\Gamma_{\tau} \frac{\alpha v}{1-\alpha v v_{\tau}} v_{y}\right) \hat{\bar{y}}_{t}^{i}+\left(\Gamma_{\theta}+\Gamma_{\tau} \frac{\alpha v}{1-\alpha v v_{\tau}} v_{\theta}\right) \hat{\bar{\theta}}^{i}\right] d t .
\end{aligned}
$$

One can easily verify that $\Gamma_{y}+\Gamma_{\tau} \frac{\alpha v}{1-\alpha v v_{\tau}} v_{y}=0$. The budget constraint then directly implies that $\hat{\theta}^{i}=0$ for the no-policy allocation. 
Allocation. The simplified (IS) equation can then be written as

$$
\dot{\hat{y}}_{t}^{i}=-G^{\prime}\left(\hat{\bar{\pi}}_{H, t}^{i}+\dot{\tilde{s}}_{t}^{i}\right)
$$

where $G^{\prime}=\frac{\Lambda_{s}}{\Lambda_{y}-\Lambda_{\tau} \frac{\alpha v}{1-\alpha v v_{\tau}} v_{y}}$. The (NKPC) becomes

$$
\dot{\bar{\pi}}_{H, t}^{i}=\rho \hat{\bar{\pi}}_{H, t}^{i}-\hat{\kappa}_{y} \hat{\bar{y}}_{t}^{i}
$$

with $\hat{\kappa}_{y}=\kappa_{y}+\kappa_{\tau} \frac{\alpha v}{1-\alpha v v_{\tau}} v_{y}$. And finally, the initial condition can be written as

$$
\hat{\bar{y}}_{0}^{i}=-\bar{G}^{\prime} \tilde{s}_{0}^{i}
$$

with $\bar{G}^{\prime}=\frac{\Lambda_{s}+\alpha v \Lambda_{\tau}}{\Lambda_{y}}$.

We can write the dynamical system describing the no-policy allocation as

$$
\dot{X}_{t}^{i}=A X_{t}^{i}+B_{t}^{i}
$$

where

$$
A=\left(\begin{array}{cc}
\rho & -\hat{\kappa}_{y} \\
-G^{\prime} & 0
\end{array}\right), \quad B_{t}^{i}=\left(\begin{array}{c}
0 \\
-G^{\prime} \dot{s}_{t}^{i}
\end{array}\right), \quad X_{0}^{i}=\left(\begin{array}{c}
\hat{\bar{T}}_{H, 0}^{i} \\
-\bar{G}^{\prime} \tilde{s}_{0}^{i}
\end{array}\right),
$$

We will solve for $\hat{\pi}_{H, 0}^{i}$ to ensure the stability of this system. It is straightforward to verify that the only negative eigenvalue of $A$ is

$$
v=\frac{1}{2}\left(\rho-\sqrt{\rho^{2}+4 G^{\prime} \hat{\kappa}_{y}}\right) .
$$

The corresponding eigenvector is $X_{v}=\left(-\frac{v}{G^{\prime}}, 1\right)^{\prime}$.

We know that the solution to this system is given by

$$
X_{t}^{i}=e^{A t}\left[X_{0}^{i}+\int_{0}^{t} e^{-A s}\left(-G^{\prime} \dot{s}_{t}^{i} E_{2}\right) d s\right],
$$

where $E_{1}=(1,0)^{\prime}$ and $E_{2}=(0,1)^{\prime}$ are projection matrices. We restrict attention to the parameter subspace where $A$ is nonsingular. Furthermore, we know that

$$
\dot{\tilde{s}}_{t}^{i}=-\psi a_{0}^{i} e^{-\psi t},
$$

where $a_{0}^{i}$ is the initial technology shock. This implies

$$
X_{t}^{i}=e^{A t}\left[X_{0}^{i}+\psi G^{\prime} a_{0}^{i} \int_{0}^{t} e^{-A s-\psi s} E_{2} d s\right]
$$




$$
=e^{A t}\left[X_{0}^{i}+\psi G^{\prime} a_{0}^{i}(A+\psi I)^{-1} E_{2}\right]-\psi G^{\prime} a_{0}^{i} e^{-\psi t}(A+\psi I)^{-1} E_{2} .
$$

Stability. For stability, we require the term in square brackets be spanned on the stable manifold, which is satisfied if and only if there exists an $\alpha_{v} \in \mathbb{C}$ such that

$$
\alpha_{v} X_{v}=X_{0}^{i}+\psi G^{\prime} a_{0}^{i}(A+\psi I)^{-1} E_{2} .
$$

This condition implies the two stability equations

$$
\begin{aligned}
-\frac{v}{G^{\prime}} \alpha_{v} & =\hat{\bar{\pi}}_{H, 0}^{i}+\psi G^{\prime} a_{0}^{i} E_{1}^{\prime}(A+\psi I)^{-1} E_{2} \\
\alpha_{v} & =-\bar{G}^{\prime} \tilde{s}_{0}^{i}+\psi G^{\prime} a_{0}^{i} E_{2}^{\prime}(A+\psi I)^{-1} E_{2},
\end{aligned}
$$

from which we can solve explicitly for $\alpha_{v}$ and $\hat{\bar{\pi}}_{H, 0}^{i}$. Note that we have

$$
\begin{aligned}
& E_{1}^{\prime}(A+\psi I)^{-1} E_{2}=\frac{\tilde{\kappa}_{y}}{\hat{\psi}} \\
& E_{2}^{\prime}(A+\psi I)^{-1} E_{2}=\frac{\rho+\psi}{\hat{\psi}},
\end{aligned}
$$

where $\hat{\psi}=(\rho+\psi) \psi-G^{\prime} \hat{\kappa}_{y}$. We can now rewrite the two stability conditions as

$$
\begin{aligned}
-\frac{v}{G^{\prime}} \alpha_{v} & =\hat{\pi}_{H, 0}^{i}+\psi G^{\prime} \frac{\hat{\kappa}_{y}}{\hat{\psi}} a_{0}^{i} \\
\alpha_{v} & =-\bar{G}^{\prime} \tilde{s}_{0}^{i}+\psi G^{\prime} \frac{\rho+\psi}{\hat{\psi}} a_{0}^{i} .
\end{aligned}
$$

We can now characterize the closed-form solution of our dynamical system, which is given by

$$
X_{t}^{i}=\alpha_{v} e^{v t} X_{v}-\psi G^{\prime} a_{0}^{i} e^{-\psi t}(A+\psi I)^{-1} E_{2}
$$

from which we can back out explicit solutions for inflation and output:

$$
\begin{aligned}
\hat{\bar{\pi}}_{H, t}^{i} & =-\frac{v}{G^{\prime}} \alpha_{\nu} e^{\nu t}-\psi G^{\prime} \frac{\hat{\kappa}_{y}}{\hat{\psi}} e^{-\psi t} a_{0}^{i} \\
\hat{\bar{y}}_{t}^{i} & =\alpha_{\nu} e^{\nu t}-\psi G^{\prime} \frac{\rho+\psi}{\hat{\psi}} e^{-\psi t} a_{0}^{i} .
\end{aligned}
$$

We note here that the no-policy benchmark allocation is $\chi$-invariant. To gain some intuition for this result, note that under Cole-Obstfeld and with profit redistribution the positive allocation is symmetric, with $\hat{\bar{c}}_{t}^{i, o}=\hat{\bar{c}}_{t}^{i, r}$, and trade is balanced in every period, $\hat{N X}_{t}^{i}=0$. That is, HtM agents and optimizers always consume the same amount and face the same budget constraint, in a sense, since 
there is no inter-temporal substitution.

\section{D.3 Loss Function and Planning Problem}

Before we can set up the planning problem for a currency union with hand-to-mouth agents, we have to derive the loss function. Our loss function features three distinct policy instruments: expost transfers, capital controls and government spending. It also leaves room for several derivative policy instruments which we will discuss later. We follow the linear-quadratic approach discussed in Benigno and Woodford (2012). We continue to assume that there are no aggregate shocks but in this section, to derive the loss function, we need to temporarily allow for idiosyncratic shocks in all countries.

\section{D.3.1 Notation}

Once again, we reiterate our notational conventions. For all $X_{t}^{i} \notin\left\{T_{t}^{i, o}, T_{t}^{i, r}, \tau_{t}^{i, o}, \tau_{t}^{i, r}, N F A_{t}^{i}, N X_{t}^{i}\right\}$, we have $x_{t}^{i}=\ln \left(X_{t}^{i}\right)-\ln \left(X^{i}\right)$, where $X^{i}$ is the steady state value of $X_{t}^{i}$; furthermore, we define $\tilde{x}_{t}^{i}=$ $\ln \left(\tilde{X}_{t}^{i}\right)-\ln \left(X^{i}\right), \hat{x}_{t}^{i}=\ln \left(X_{t}^{i}\right)-\ln \left(\tilde{X}_{t}^{i}\right)$ and $\hat{\bar{x}}_{t}^{i}=\hat{x}_{t}^{i}-\int_{0}^{1} \hat{x}_{t}^{i} d i \equiv \hat{x}_{t}^{i}-\hat{x}_{t}^{*}$. Our goal is to approximate welfare in terms of $\hat{\bar{x}}_{t}^{i}$ variables.

By definition, we have $X_{t}^{i}=X^{i} e^{x_{t}^{i}}$. Taylor expansions of the function $x_{t}^{i} \mapsto X_{t}^{i}$ around $x_{t}^{i}=0$ yield:

To $1^{\text {st }}$ order: $X_{t}^{i} \approx X^{i}+X^{i} x_{t}^{i}=X^{i}\left(1+x_{t}^{i}\right)$

To $2^{\text {nd }}$ order: $X_{t}^{i} \approx X^{i}+X^{i} x_{t}^{i}+\frac{1}{2} X^{i}\left(x_{t}^{i}\right)^{2}=X^{i}\left(1+x_{t}^{i}+\frac{1}{2}\left(x_{t}^{i}\right)^{2}\right)$.

So for example, the second-order approximation of $\ln \left(X_{t}^{i}\right)=\ln \left(X^{i}\right)+x_{t}^{i}$ around $x_{t}^{i}=0$ is exactly $\ln \left(X^{i}\right)+x_{t}^{i}$. Similarly, approximating $\left(X_{t}^{i}\right)^{\phi}=\left(X^{i}\right)^{\phi} e^{\phi x_{t}^{i}}$ around $\phi x_{t}^{i}=0$, we have

$$
\left(X_{t}^{i}\right)^{\phi} \approx\left(X^{i}\right)^{\phi}+\frac{\left(X^{i}\right)^{\phi} e^{0}}{1}+\frac{\left(X^{i}\right)^{\phi} e^{0}}{2}\left(\phi x_{t}^{i}\right)^{2}=\left(X^{i}\right)^{\phi}\left(1+\phi x_{t}^{i}+\frac{\phi^{2}}{2}\left(x_{t}^{i}\right)^{2}\right) .
$$

\section{D.3.2 Starting with Household Utility}

We begin by taking as our welfare criterion the households' utility function. In particular, we solve a coordinated problem, with the social planner's objective function of the form

$$
\begin{aligned}
U=\int_{0}^{\infty} \int_{0}^{1} e^{-\rho t}[ & \chi(1-v) \ln \left(C_{t}^{i, r}\right)-\frac{\chi}{1+\phi}\left(N_{t}^{i, r}\right)^{1+\phi} \\
& \left.+(1-\chi)(1-v) \ln \left(C_{t}^{i, o}\right)-\frac{1-\chi}{1+\phi}\left(N_{t}^{i, o}\right)^{1+\phi}+v \ln \left(G_{t}^{i}\right)\right] d i d t
\end{aligned}
$$


Approximating the RHS to second order, we have

$$
\begin{aligned}
& U=\int_{0}^{\infty} \int_{0}^{1} e^{-\rho t}\left[\chi(1-v) \ln \left(C^{i, r}\right)+\chi(1-v) c_{t}^{i, r}+(1-\chi)(1-v) \ln \left(C^{i, o}\right)+(1-\chi)(1-v) c_{t}^{i, o}\right. \\
&-\frac{\chi}{1+\phi}\left(N^{i, r}\right)^{1+\phi}\left(1+(1+\phi) n_{t}^{i, r}+\frac{(1+\phi)^{2}}{2}\left(n_{t}^{i, r}\right)^{2}\right) \\
&\left.-\frac{1-\chi}{1+\phi}\left(N^{i, o}\right)^{1+\phi}\left(1+(1+\phi) n_{t}^{i, o}+\frac{(1+\phi)^{2}}{2}\left(n_{t}^{i, o}\right)^{2}\right)+v \ln \left(G^{i}\right)+v g_{t}^{i}\right] d i d t .
\end{aligned}
$$

Recall the steady state relations: $Y^{i}=1, C^{i, r}=C^{i, o}=(1-v), G^{i}=v$, and $\left(N^{i, o}\right)^{1+\phi}=$ $\left(N^{i, r}\right)^{1+\phi}=1$. Hence, we have

$$
\begin{aligned}
U=\int_{0}^{\infty} \int_{0}^{1} e^{-\rho t}[ & (1-v) \ln (1-v)-\frac{1}{1+\phi}+v \ln (v) \\
& +(1-v)\left[\chi c_{t}^{i, r}+(1-\chi) c_{t}^{i, o}\right]-\left[\chi n_{t}^{i, r}+(1-\chi) n_{t}^{i, o}\right]+v g_{t}^{i} \\
& \left.-\frac{\chi(1+\phi)}{2}\left(n_{t}^{i, r}\right)^{2}-\frac{(1-\chi)(1+\phi)}{2}\left(n_{t}^{i, o}\right)^{2}\right] \text { didt } .
\end{aligned}
$$

Let $Z^{i} \equiv(1-v) \ln (1-v)-\frac{1}{1+\phi}+v \ln (v)$. Also note that $\chi c_{t}^{i, r}+(1-\chi) c_{t}^{i, o}=c_{t}^{i}$ and $\chi n_{t}^{i, r}+(1-$ $\chi) n_{t}^{i, o}=n_{t}^{i}$. Finally, we use the linearity of the integral operator, noting that $\int_{0}^{1} x_{t}^{i} d i \equiv \int_{0}^{1}\left(\hat{x}_{t}^{i}+\hat{x}_{t}^{*}+\right.$ $\left.\tilde{x}_{t}^{i}\right) d i=\hat{x}_{t}^{*}$, to write

$$
U=\int_{0}^{\infty} \int_{0}^{1} e^{-\rho t}\left[Z^{i}+(1-v) \hat{c}_{t}^{*}+v \hat{g}_{t}^{*}-n_{t}^{i}-\frac{\chi(1+\phi)}{2}\left(n_{t}^{i, r}\right)^{2}-\frac{(1-\chi)(1+\phi)}{2}\left(n_{t}^{i, o}\right)^{2}\right] d i d t .
$$

\section{D.3.3 Linear-Quadratic Framework}

Having completed a second-order approximation of the social planner's welfare criterion, we will now try to express said metric in terms of the variables $\hat{\bar{y}}_{t}^{i}, \hat{\bar{\pi}}_{H, t}^{i}, \hat{\bar{\tau}}_{t}^{i, r}, \hat{\bar{\theta}}_{t}^{i}$, and $\hat{\bar{g}}_{t}^{i}$, where the latter three represent the full set of policy instruments available to the planner. In this particular case, a proper linear-quadratic approximation rests on the following two key steps:

i. Approximate the A-D relation to second order to find an expression for $(1-v) \hat{c}_{t}^{*}+v \hat{g}_{t}^{*}$.

ii. Express $\hat{n}_{t}^{i}$ in terms of inflation.

We start with (2): With Calvo-pricing, we have the distorted production function

$$
Y_{t}^{i} \int_{0}^{1}\left(\frac{P_{H, t}^{i}(j)}{P_{H, t}^{i}}\right)^{-\epsilon} d j=A_{t}^{i} N_{t}^{i} .
$$

Therefore, we can approximate $n_{t}^{i}=y_{t}^{i}+z_{t}^{i}-a_{t}^{i}$ to second order, where

$$
z_{t}^{i}=\ln \left(\int_{0}^{1}\left(\frac{P_{H, t}^{i}(j)}{P_{H, t}^{i}}\right)^{-\epsilon} d j\right) \approx \frac{\epsilon}{2} \int_{0}^{1}\left(P_{H, t}^{i}(j)-P_{H, t}^{i}\right)^{2} d j=\alpha_{\pi}\left(\pi_{H, t}^{i}\right)^{2},
$$


for $\alpha_{\pi}=\frac{\epsilon}{2 \lambda}$ and $\lambda=\rho_{\delta}\left(\rho+\rho_{\delta}\right)$. Note that this definition of $\alpha_{\pi}$ differs slightly from that used in the main body of the paper. We found this notation slightly more convenient for the analysis in the appendix. Hence, we have

$$
n_{t}^{i}=y_{t}^{i}+\alpha_{\pi}\left(\pi_{H, t}^{i}\right)^{2}-a_{t}^{i} .
$$

The technology term is invariant to policy and so we drop it. It would later naturally drop out as we rewrite the loss in gaps from the natural allocation.

Next, we tackle the more difficult step (1): Recall that the A-D relation can be written as

$$
Y_{t}^{i}-G_{t}^{i}=S_{t}^{i} C^{*, o}\left((1-\alpha) \frac{\Theta_{t}^{i} C_{t}^{i}}{C_{t}^{i, o}}+\alpha \int_{0}^{1} \frac{\Theta_{t}^{j} C_{t}^{j}}{C_{t}^{j, o}} d j\right)
$$

The linear-quadratic procedure requires that we approximate all those constraints to second order that we use to substitute out terms in the loss function. We start by approximating the LHS to second order. We have

$$
\begin{aligned}
L H S & \approx\left(1+y_{t}^{i}+\frac{1}{2}\left(y_{t}^{i}\right)^{2}\right)-v\left(1+g_{t}^{i}+\frac{1}{2}\left(g_{t}^{i}\right)^{2}\right) \\
& =(1-v)+\left[\hat{y}_{t}^{i}+\tilde{y}_{t}^{i}\right]+\frac{1}{2}\left[\hat{y}_{t}^{i}+\tilde{y}_{t}^{i}\right]^{2}-v\left[\hat{g}_{t}^{i}+\tilde{g}_{t}^{i}\right]-\frac{v}{2}\left[\hat{g}_{t}^{i}+\tilde{g}_{t}^{i}\right]^{2} .
\end{aligned}
$$

Integrating (using linearity of the integral), noting that $\tilde{y}_{t}^{*}=0$ and $\tilde{g}_{t}^{i}=a_{t}^{i}$ because we only consider idiosyncratic shocks (and country $i$ is of measure 0 ) and we defined the natural allocation without policy intervention, respectively, we have

$$
L H S \approx(1-v) Y^{i}+Y^{i} \hat{y}_{t}^{*}+\frac{Y^{i}}{2} \int_{0}^{1}\left[\hat{y}_{t}^{i}+\tilde{y}_{t}^{i}\right]^{2} d i-v Y^{i} \hat{g}_{t}^{*}-\frac{v Y^{i}}{2} \int_{0}^{1}\left[\hat{g}_{t}^{i}+\tilde{g}_{t}^{i}\right]^{2} d i .
$$

For the RHS, we have

$$
\begin{aligned}
R H S \approx & (1-\alpha) \frac{S^{i} C^{*, o} \Theta^{i} C^{i}}{C^{i, o}}\left(1+s_{t}^{i}+c_{t}^{*, o}+\theta_{t}^{i}+c_{t}^{i}-c_{t}^{i, o}+\frac{1}{2}\left[s_{t}^{i}+c_{t}^{*, o}+\theta_{t}^{i}+c_{t}^{i}-c_{t}^{i, o}\right]^{2}\right) \\
& +\alpha \int_{0}^{1} \frac{\Theta^{j} C^{j} S^{i} C^{*, o}}{C^{j, o}}\left(1+s_{t}^{i}+c_{t}^{*, o}+\theta_{t}^{j}+c_{t}^{j}-c_{t}^{j, o}+\frac{1}{2}\left[s_{t}^{i}+c_{t}^{*, o}+\theta_{t}^{j}+c_{t}^{j}-c_{t}^{j, o}\right]^{2}\right) d j \\
= & (1-\alpha)(1-v) Y^{i}\left(1+s_{t}^{i}+c_{t}^{*, o}+\theta_{t}^{i}+c_{t}^{i}-c_{t}^{i, o}+\frac{1}{2}\left[s_{t}^{i}+c_{t}^{*, o}+\theta_{t}^{i}+c_{t}^{i}-c_{t}^{i, o}\right]^{2}\right) \\
& +\alpha(1-v) Y^{i}\left(1+\theta_{t}^{*}+c_{t}^{*}+s_{t}^{*}+\frac{1}{2} \int_{0}^{1}\left[s_{t}^{i}+c_{t}^{*, o}+\theta_{t}^{j}+c_{t}^{j}-c_{t}^{j, o}\right]^{2} d j\right) .
\end{aligned}
$$

Putting these together, we can write

$$
\begin{aligned}
& \hat{y}_{t}^{*}-v \hat{g}_{t}^{*}+\frac{1}{2} \int_{0}^{1}\left[\hat{y}_{t}^{i}+\tilde{y}_{t}^{i}\right]^{2} d i-\frac{v}{2} \int_{0}^{1}\left[\hat{g}_{t}^{i}+\tilde{g}_{t}^{i}\right]^{2} d i \\
= & (1-v) c_{t}^{*}+(1-v) s_{t}^{*}+(1-v) \theta_{t}^{*}
\end{aligned}
$$




$$
\begin{aligned}
& +\frac{1-v}{2} \int_{0}^{1}\left(\theta_{t}^{i}+c_{t}^{i}-c_{t}^{i, o}+c_{t}^{*, o}\right)^{2} d i \\
& +\frac{(1-\alpha)(1-v)}{2} \int_{0}^{1}\left(\left(s_{t}^{i}\right)^{2}+2 s_{t}^{i}\left(\theta_{t}^{i}+c_{t}^{i}-c_{t}^{i, o}+c_{t}^{*, o}\right)\right) d i \\
& +\frac{\alpha(1-v)}{2} \int_{0}^{1} \int_{0}^{1}\left(\left(s_{t}^{i}\right)^{2}+2 s_{t}^{i}\left(\theta_{t}^{j}+c_{t}^{j}-c_{t}^{j, o}+c_{t}^{*, o}\right)\right) d j d i .
\end{aligned}
$$

\section{Re-writing,}

$$
\begin{aligned}
& \hat{y}_{t}^{*}-v \hat{g}_{t}^{*}+\frac{1}{2} \int_{0}^{1}\left(y_{t}^{i}\right)^{2} d i-\frac{v}{2} \int_{0}^{1}\left(g_{t}^{i}\right)^{2} d i \\
= & (1-v) c_{t}^{*}+(1-v) s_{t}^{*}+(1-v) \theta_{t}^{*} \\
& +\frac{1-v}{2} \int_{0}^{1}\left(\left(\theta_{t}^{i}\right)^{2}+\left(c_{t}^{i}\right)^{2}+\left(c_{t}^{i, o}\right)^{2}+\left(c_{t}^{*, o}\right)^{2}+2 \theta_{t}^{i} c_{t}^{i}+2 \theta_{t}^{i} c_{t}^{*, o}-2 \theta_{t}^{i} c_{t}^{i, o}-2 c_{t}^{i} c_{t}^{i, o}+2 c_{t}^{i} c_{t}^{*, o}-2 c_{t}^{i, o} c_{t}^{*, o}\right) d i \\
& +(1-\alpha)(1-v) s_{t}^{*} c_{t}^{*, o}+\frac{(1-\alpha)(1-v)}{2} \int_{0}^{1}\left(s_{t}^{i}\right)^{2} d i+(1-\alpha)(1-v) \int_{0}^{1} s_{t}^{i}\left(\theta_{t}^{i}+c_{t}^{i}-c_{t}^{i, o}+c_{t}^{*, o}\right) d i \\
& +\frac{\alpha(1-v)}{2} \int_{0}^{1}\left(s_{t}^{i}\right)^{2} d i+\alpha(1-v)\left(\theta_{t}^{*}+c_{t}^{*}\right) \int_{0}^{1} s_{t}^{i} d i .
\end{aligned}
$$

Note that by definition, we have for all union variables $x_{t}^{*}=\hat{x}_{t}^{*}+\tilde{x}_{t}^{*}=\hat{x}_{t}^{*}$ because $\tilde{x}_{t}^{*}=0$ (we use idiosyncratic shocks and country $i$ is of measure 0 ). Therefore, we also have $\hat{s}_{t}^{*}=s_{t}^{*}=0$ and $\hat{\theta}_{t}^{*}=\theta_{t}^{*}=0$. Hence, we have

$$
\begin{aligned}
& \hat{y}_{t}^{*}-v \hat{g}_{t}^{*}+\frac{1}{2} \int_{0}^{1}\left(y_{t}^{i}\right)^{2} d i-\frac{v}{2} \int_{0}^{1}\left(g_{t}^{i}\right)^{2} d i \\
= & (1-v) \hat{c}_{t}^{*}+\frac{1-v}{2}\left(\hat{c}_{t}^{*, o}\right)^{2}-(1-v)\left(\hat{c}_{t}^{*, o}\right)^{2}+(1-v) \hat{c}_{t}^{*} \hat{c}_{t}^{*, o} \\
& +\frac{1-v}{2} \int_{0}^{1}\left(\left(\theta_{t}^{i}\right)^{2}+\left(c_{t}^{i}\right)^{2}+\left(c_{t}^{i, o}\right)^{2}+2 \theta_{t}^{i} c_{t}^{i}-2 \theta_{t}^{i} c_{t}^{i, o}-2 c_{t}^{i} c_{t}^{i, o}\right) d i \\
& +\frac{1-v}{2} \int_{0}^{1}\left(s_{t}^{i}\right)^{2} d i+(1-\alpha)(1-v) \int_{0}^{1} s_{t}^{i}\left(\theta_{t}^{i}+c_{t}^{i}-c_{t}^{i, o}\right) d i .
\end{aligned}
$$

And so finally, we have

$$
\begin{aligned}
(1-v) \hat{c}_{t}^{*}+v \hat{g}_{t}^{*}= & \hat{y}_{t}^{*}+\frac{1}{2} \int_{0}^{1}\left(y_{t}^{i}\right)^{2} d i-\frac{v}{2} \int_{0}^{1}\left(g_{t}^{i}\right)^{2} d i-(1-v) \hat{c}_{t}^{*} \hat{c}_{t}^{*, o} \\
& +\frac{1-v}{2}\left(\hat{c}_{t}^{*, o}\right)^{2}-\frac{1-v}{2} \int_{0}^{1}\left(\left(\theta_{t}^{i}\right)^{2}+\left(c_{t}^{i}\right)^{2}+\left(c_{t}^{i, o}\right)^{2}+2 \theta_{t}^{i} c_{t}^{i}-2 \theta_{t}^{i} c_{t}^{i, o}-2 c_{t}^{i} c_{t}^{i, o}\right) d i \\
& -\frac{1-v}{2} \int_{0}^{1}\left(s_{t}^{i}\right)^{2} d i-(1-\alpha)(1-v) \int_{0}^{1} s_{t}^{i}\left(\theta_{t}^{i}+c_{t}^{i}-c_{t}^{i, o}\right) d i .
\end{aligned}
$$

Therefore, we can write the welfare criterion as

$$
U=\int_{0}^{\infty} e^{-\rho t}\left[-\alpha_{\pi}\left(\pi_{H, t}^{i}\right)^{2}+U_{t}^{i}\right] d t
$$


where $\int_{0}^{1} \hat{y}_{t}^{*}-y_{t}^{i} d i=0$ and thus drops out, and so

$$
\begin{aligned}
U_{t}^{i}= & Z^{i}+\frac{1-v}{2}\left(\hat{c}_{t}^{*, o}\right)^{2}-(1-v) \hat{c}_{t}^{*} \hat{c}_{t}^{*, o} \\
& +\frac{1}{2}\left(y_{t}^{i}\right)^{2}-\frac{v}{2}\left(g_{t}^{i}\right)^{2}-\frac{1-v}{2}\left(s_{t}^{i}\right)^{2}-(1-\alpha)(1-v) s_{t}^{i}\left(\theta_{t}^{i}+c_{t}^{i}-c_{t}^{i, o}\right) \\
& -\frac{1-v}{2}\left(\left(\theta_{t}^{i}\right)^{2}+\left(c_{t}^{i}\right)^{2}+\left(c_{t}^{i, o}\right)^{2}+2 \theta_{t}^{i} c_{t}^{i}-2 \theta_{t}^{i} c_{t}^{i, o}-2 c_{t}^{i} c_{t}^{i, o}\right) \\
& -\frac{\chi(1+\phi)}{2}\left(n_{t}^{i, r}\right)^{2}-\frac{(1-\chi)(1+\phi)}{2}\left(n_{t}^{i, o}\right)^{2} .
\end{aligned}
$$

\section{D.3.4 Gap Notation}

We have now finished deriving a second-order approximation of the social planner's welfare criterion. We want to express this equation entirely in gap notation because we later want to write all optimal policy problems in gap notation as well.

To that end, we convert variables according to $x_{t}^{i}=\hat{x}_{t}^{i}+\hat{x}_{t}^{*}+\tilde{x}_{t}^{i}$. After some algebra, we find

$$
\begin{aligned}
& U_{t}^{i}=Z^{i}+\hat{Z}_{t}^{*}+\tilde{Z}_{t}^{i}+\frac{1}{2}\left(\hat{\bar{y}}_{t}^{i}\right)^{2}+\hat{\bar{y}}_{t}^{i} \tilde{y}_{t}^{i}-\frac{v}{2}\left(\hat{\bar{g}}_{t}^{i}\right)^{2}-v \hat{\bar{g}}_{t}^{i} \tilde{g}_{t}^{i}-\frac{1-v}{2}\left(\hat{\bar{s}}_{t}^{i}\right)^{2}-(1-v) \hat{\bar{s}}_{t}^{i} \tilde{s}_{t}^{i} \\
& -(1-\alpha)(1-v) \hat{\bar{s}}_{t}^{i}\left[\hat{\theta}_{t}^{i}+\tilde{\theta}_{t}^{i}+\hat{\bar{c}}_{t}^{i}+\tilde{c}_{t}^{i}-\hat{\bar{c}}_{t}^{i, o}-\tilde{c}_{t}^{i, o}\right]-(1-\alpha)(1-v) \tilde{s}_{t}^{i}\left[\hat{\theta}_{t}^{i}+\hat{\bar{c}}_{t}^{i}-\hat{\bar{c}}_{t}^{i, o}\right] \\
& -\frac{1-v}{2}\left(\left(\hat{\bar{\theta}}_{t}^{i}\right)^{2}+2 \hat{\theta}_{t}^{i} \tilde{\theta}_{t}^{i}+\left(\hat{\bar{c}}_{t}^{i}\right)^{2}+2 \hat{\bar{c}}_{t} \tilde{c}_{t}^{i}+\left(\hat{\bar{c}}_{t}^{i, o}\right)^{2}+2 \hat{\bar{c}}_{t}^{i, o} \widetilde{c}_{t}^{i, o}+2 \hat{\bar{\theta}}_{t}^{i} \hat{\bar{c}}_{t}^{i}+2 \hat{\theta}_{t}^{i} \tilde{c}_{t}^{i}+2 \tilde{\theta}_{t}^{i} \hat{\bar{c}}_{t}^{i}\right. \\
& \left.-2 \hat{\bar{\theta}}_{t}^{i} \hat{\bar{c}}_{t}^{i, o}-2 \hat{\bar{\theta}}_{t}^{i} \tilde{c}_{t}^{i, o}-2 \tilde{\theta}_{t}^{i} \hat{\bar{c}}_{t}^{i, o}-2 \hat{\bar{c}}_{t}^{i} \hat{\bar{c}}_{t}^{i, o}-2 \hat{\bar{c}}_{t}^{i} \tilde{c}_{t}^{i, o}-2 \tilde{c}_{t}^{i} \hat{\bar{c}}_{t}^{i, o}\right) \\
& -\frac{\chi(1+\phi)}{2}\left(\hat{\bar{n}}_{t}^{i, r}\right)^{2}-\frac{\chi(1+\phi)}{2} \hat{\bar{n}}_{t}^{i, r} \tilde{n}_{t}^{i, r}-\frac{(1-\chi)(1+\phi)}{2}\left(\hat{\bar{n}}_{t}^{i, o}\right)^{2}-\frac{(1-\chi)(1+\phi)}{2} \hat{n}_{t}^{i, o} \tilde{n}_{t}^{i, o},
\end{aligned}
$$

where

$$
\begin{aligned}
\hat{Z}_{t}^{*}= & \frac{1}{2}\left(\hat{y}_{t}^{*}\right)^{2}-\frac{v}{2}\left(\hat{g}_{t}^{*}\right)^{2}-\frac{1-v}{2}\left(\hat{c}_{t}^{*}\right)^{2}-\frac{\chi(1+\phi)}{2}\left(\hat{n}_{t}^{*, r}\right)^{2}-\frac{(1-\chi)(1+\phi)}{2}\left(\hat{n}_{t}^{*, o}\right)^{2} \\
\tilde{Z}_{t}^{i}= & \frac{1}{2}\left(\tilde{y}_{t}^{i}\right)^{2}-\frac{v}{2}\left(\tilde{g}_{t}^{i}\right)^{2}-\frac{1-v}{2}\left(\tilde{s}_{t}^{i}\right)^{2}-(1-\alpha)(1-v) \tilde{s}_{t}^{i}\left[\tilde{\theta}_{t}^{i}+\hat{c}_{t}^{*}+\tilde{c}_{t}^{i}-\hat{c}_{t}^{*, o}-\tilde{c}_{t}^{i, o}\right]-\frac{1-v}{2}\left(\tilde{\theta}_{t}^{i}\right)^{2}-\frac{1-v}{2}\left(\tilde{c}_{t}^{i}\right)^{2} \\
& -\frac{1-v}{2}\left(\tilde{c}_{t}^{i, o}\right)^{2}-(1-v) \tilde{\theta}_{t}^{i}\left(\hat{c}_{t}^{*}+\tilde{c}_{t}^{i}\right)+(1-v) \tilde{\theta}_{t}^{i}\left(\hat{c}_{t}^{*, o}+\tilde{c}_{t}^{i, o}\right)+(1-v)\left[\tilde{c}_{t}^{i} \tilde{c}_{t}^{i, o}+\hat{c}_{t}^{*} \tilde{c}_{t}^{i, o}+\tilde{c}_{t}^{i} \hat{c}_{t}^{*, o}\right] \\
& -\frac{\chi(1+\phi)}{2}\left(\tilde{n}_{t}^{i, r}\right)^{2}-\frac{(1-\chi)(1+\phi)}{2}\left(\tilde{n}_{t}^{i, o}\right)^{2} .
\end{aligned}
$$

We also use gap notation for the inflation term in the loss function, noting that $\pi_{H, t}^{i}=\hat{\pi}_{H, t}^{i}=$ $\hat{\pi}_{H, t}^{i}+\hat{\pi}_{H, t}^{*}$. As in the body of the paper, it is easy to verify that the social planner can fully stabilize union-wide inflation so that $\hat{\pi}_{H, t}^{*}=0$. Alternatively, we could have added terms in $\hat{\pi}_{H, t}^{*}$ to the term $\hat{Z}_{t}^{*}$ which will be entirely inconsequential for all subsequent analysis since the planning problem can be disaggregated by country, as in the body of the paper, and we will focus exclusively on idiosyncratic shocks in country $i$. 


\section{D.3.5 Final Steps}

What remains now is to use the first-order approximation of the equilibrium conditions to solve out terms so that we can write the welfare criterion entirely as a function of $\hat{\bar{y}}_{t}^{i}, \hat{\bar{\pi}}_{H, t}^{i}, \hat{\bar{\tau}}_{t}^{i, r}, \hat{\bar{\theta}}_{t}^{i}$, and $\hat{\bar{g}}_{t}^{i}$. This is consistent with the linear-quadratic approach.

Drawing on our earlier derivations, we use the following substitutions:

$$
\begin{aligned}
\hat{\bar{n}}_{t}^{i, r} & =\eta_{y} \hat{\bar{y}}_{t}^{i}+\eta_{g} \hat{\bar{g}}_{t}^{i}+\eta_{\theta} \hat{\bar{\theta}}_{t}^{i}+\eta_{\tau} \hat{\bar{\tau}}_{t}^{i, r} \\
\hat{\bar{n}}_{t}^{i, o} & =\left(\frac{1}{1-\chi}-\frac{\chi}{1-\chi} \eta_{y}\right) \hat{\bar{y}}_{t}^{i}-\frac{\chi}{1-\chi} \eta_{g} \hat{\bar{g}}_{t}^{i}-\frac{\chi}{1-\chi} \eta_{\theta} \hat{\bar{\theta}}_{t}^{i}-\frac{\chi}{1-\chi} \eta_{\tau} \hat{\bar{\tau}}_{t}^{i, r} \\
\hat{\bar{c}}_{t}^{i, r} & =\psi_{y} \hat{\bar{y}}_{t}^{i}+\psi_{\theta} \hat{\bar{\theta}}_{t}^{i}+\psi_{\tau} \hat{\bar{\tau}}_{t}^{i, r}+\psi_{g} \hat{\bar{g}}_{t}^{i} \\
\hat{\bar{c}}_{t}^{i, o} & =(1-\alpha) \nu_{y} \hat{\bar{y}}_{t}^{i}+\left[1+(1-\alpha) v_{\theta}\right] \hat{\bar{\theta}}_{t}^{i}+(1-\alpha) \nu_{g} \hat{\bar{g}}_{t}^{i}+(1-\alpha) \nu_{\tau} \hat{\bar{\tau}}_{t}^{i, r} \\
\hat{\bar{c}}_{t}^{i} & =\zeta_{y} \hat{\bar{y}}_{t}^{i}+\zeta_{\theta} \hat{\bar{\theta}}_{t}^{i}+\zeta_{\tau} \hat{\bar{\tau}}_{t}^{i, r}+\zeta_{g} \hat{\bar{g}}_{t}^{i} .
\end{aligned}
$$

After a lot of algebra, the social planner's welfare criterion can be written as

$$
\begin{aligned}
U=\int_{0}^{\infty} e^{-\rho t} \int_{0}^{1}[ & -\alpha_{\pi}\left(\hat{\bar{\tau}}_{H, t}^{i}\right)^{2}+Z^{i}+\hat{Z}_{t}^{*}+\tilde{Z}_{t}^{i}+\alpha_{y y}\left(\hat{\bar{y}}_{t}^{i}\right)^{2}+\alpha_{\theta \theta}\left(\hat{\bar{\theta}}_{t}^{i}\right)^{2}+\alpha_{\tau \tau}\left(\hat{\bar{\tau}}_{t}^{i, r}\right)^{2}+\alpha_{g g}\left(\hat{\bar{g}}_{t}^{i}\right)^{2} \\
& +\alpha_{y \theta} \hat{\bar{y}}_{t}^{i} \hat{\bar{\theta}}_{t}^{i}+\alpha_{y g} \hat{\bar{y}}_{t}^{i} \hat{\bar{g}}_{t}^{i}+\alpha_{y \tau} \hat{\bar{y}}_{t}^{i} \hat{\bar{\tau}}_{t}^{i, r}+\alpha_{g \theta} \hat{\bar{g}}_{t}^{i} \hat{\bar{\theta}}_{t}^{i}+\alpha_{g \tau} \hat{\bar{g}}_{t}^{i} \hat{\bar{\tau}}_{t}^{i, r}+\alpha_{\tau \theta} \hat{\bar{\tau}}_{t}^{i, r} \hat{\bar{\theta}}_{t}^{i} \\
& \left.+\alpha_{y, t}^{i} \hat{\bar{y}}_{t}^{i}+\alpha_{g, t}^{i} \hat{\bar{g}}_{t}^{i}+\alpha_{\tau, t}^{i} \hat{\bar{\tau}}_{t}^{i}+\alpha_{\theta, t}^{i} \hat{\bar{\theta}}_{t}^{i}\right] d i d t,
\end{aligned}
$$

where

$$
\begin{aligned}
& \alpha_{y y}=\frac{1}{2}-\frac{1-v}{2} v_{y}^{2}-(1-\alpha)(1-v) v_{y}\left(\zeta_{y}-(1-\alpha) v_{y}\right)-\frac{1-v}{2} \zeta_{y}^{2}-\frac{1-v}{2}(1-\alpha)^{2} v_{y}^{2} \\
& +(1-v)(1-\alpha) v_{y} \zeta_{y}-\frac{\chi(1+\phi)}{2} \eta_{y}^{2}-\frac{(1-\chi)(1+\phi)}{2}\left(\frac{1}{1-\chi}-\frac{\chi}{1-\chi} \eta_{y}\right)^{2} \\
& \alpha_{\theta \theta}=-\frac{1-v}{2} v_{\theta}^{2}-(1-\alpha)(1-v) v_{\theta}\left(\zeta_{\theta}-(1-\alpha) v_{\theta}\right)-\frac{1-v}{2}-\frac{1-v}{2} \zeta_{\theta}^{2}-\frac{1-v}{2}\left[(1-\alpha) v_{\theta}+1\right]^{2} \\
& -(1-v)\left(\zeta_{\theta}-\left[1+(1-\alpha) v_{\theta}\right]\right)+(1-v)\left[1+(1-\alpha) v_{\theta}\right] \zeta_{\theta}-\frac{\chi(1+\phi)}{2} \eta_{\theta}^{2}-\frac{(1-\chi)(1+\phi)}{2}\left(\frac{\chi}{1-\chi} \eta_{\theta}\right)^{2} \\
& \alpha_{\tau \tau}=-\frac{1-v}{2} v_{\tau}^{2}-(1-\alpha)(1-v) \nu_{\tau}\left(\zeta_{\tau}-(1-\alpha) v_{\tau}\right)-\frac{1-v}{2} \zeta_{\tau}^{2}-\frac{1-v}{2}(1-\alpha)^{2} v_{\tau}^{2} \\
& +(1-v)(1-\alpha) v_{\tau} \zeta_{\tau}-\frac{\chi(1+\phi)}{2} \eta_{\tau}^{2}-\frac{(1-\chi)(1+\phi)}{2}\left(\frac{\chi}{1-\chi} \eta_{\tau}\right)^{2} \\
& \alpha_{g g}=-\frac{v}{2}-\frac{1-v}{2} v_{g}^{2}-(1-\alpha)(1-v) v_{g}\left(\zeta_{g}-(1-\alpha) v_{g}\right)-\frac{1-v}{2} \zeta_{g}^{2}-\frac{1-v}{2}(1-\alpha)^{2} v_{g}^{2} \\
& +(1-v)(1-\alpha) v_{g} \zeta_{g}-\frac{\chi(1+\phi)}{2} \eta_{g}^{2}-\frac{(1-\chi)(1+\phi)}{2}\left(\frac{\chi}{1-\chi} \eta_{g}\right)^{2} \\
& \alpha_{y \theta}=-(1-v) v_{y} v_{\theta}-(1-\alpha)(1-v)\left(v_{y}\left[\zeta_{\theta}-(1-\alpha) v_{\theta}\right]+v_{\theta}\left[\zeta_{y}-(1-\alpha) v_{y}\right]\right) \\
& -(1-v) \zeta_{y} \zeta_{\theta}-(1-v)(1-\alpha) v_{y}\left[1+(1-\alpha) v_{\theta}\right]-(1-v)\left(\zeta_{y}-(1-\alpha) v_{y}\right)
\end{aligned}
$$




$$
\begin{aligned}
& +(1-v)(1-\alpha) v_{y} \zeta_{\theta}+(1-v)\left[1+(1-\alpha) v_{\theta}\right] \zeta_{y}-\chi(1+\phi) \eta_{y} \eta_{\theta}-(1+\phi) \chi \eta_{\theta}\left(\frac{1}{1-\chi}-\frac{\chi}{1-\chi} \eta_{y}\right) \\
& \alpha_{y g}=-(1-v) v_{y} v_{g}-(1-\alpha)(1-v)\left(v_{y}\left[\zeta_{g}-(1-\alpha) v_{g}\right]+v_{g}\left[\zeta_{y}-(1-\alpha) v_{y}\right]\right) \\
& -(1-v) \zeta_{y} \zeta_{g}-(1-v)(1-\alpha)^{2} v_{y} v_{g}+(1-v)(1-\alpha) v_{y} \zeta_{g}+(1-v)(1-\alpha) v_{g} \zeta_{y} \\
& -\chi(1+\phi) \eta_{y} \eta_{g}-(1+\phi) \chi \eta_{g}\left(\frac{1}{1-\chi}-\frac{\chi}{1-\chi} \eta_{y}\right) \\
& \alpha_{y \tau}=-(1-v) v_{y} v_{\tau}-(1-\alpha)(1-v)\left(v_{y}\left[\zeta_{\tau}-(1-\alpha) v_{\tau}\right]+v_{\tau}\left[\zeta_{y}-(1-\alpha) v_{y}\right]\right) \\
& -(1-v) \zeta_{y} \zeta_{\tau}-(1-v)(1-\alpha)^{2} v_{y} v_{\tau}+(1-v)(1-\alpha) v_{y} \zeta_{\tau}+(1-v)(1-\alpha) \nu_{\tau} \zeta_{y} \\
& -\chi(1+\phi) \eta_{y} \eta_{\tau}-(1+\phi) \chi \eta_{\tau}\left(\frac{1}{1-\chi}-\frac{\chi}{1-\chi} \eta_{y}\right) \\
& \alpha_{g \theta}=-(1-v) v_{g} v_{\theta}-(1-\alpha)(1-v)\left(v_{g}\left[\zeta_{\theta}-(1-\alpha) v_{\theta}\right]+v_{\theta}\left[\zeta_{g}-(1-\alpha) v_{g}\right]\right) \\
& -(1-v) \zeta_{g} \zeta_{\theta}-(1-v)(1-\alpha) v_{g}\left[1+(1-\alpha) v_{\theta}\right]-(1-v)\left(\zeta_{g}-(1-\alpha) v_{g}\right) \\
& +(1-v)\left[1+(1-\alpha) v_{\theta}\right] \zeta_{g}+(1-v)(1-\alpha) v_{g} \zeta_{\theta}-\chi(1+\phi) \eta_{g} \eta_{\theta}-(1+\phi) \frac{\chi^{2}}{1-\chi} \eta_{\theta} \eta_{g} \\
& \alpha_{g \tau}=-(1-v) v_{g} v_{\tau}-(1-\alpha)(1-v)\left(v_{g}\left[\zeta_{\tau}-(1-\alpha) v_{\tau}\right]+v_{\tau}\left[\zeta_{g}-(1-\alpha) v_{g}\right]\right) \\
& -(1-v) \zeta_{g} \zeta_{\tau}-(1-v)(1-\alpha)^{2} v_{g} v_{\tau}+(1-v)(1-\alpha) v_{g} \zeta_{\tau}+(1-v)(1-\alpha) v_{\tau} \zeta_{g} \\
& -\chi(1+\phi) \eta_{g} \eta_{\tau}-(1+\phi) \frac{\chi^{2}}{1-\chi} \eta_{g} \eta_{\tau} \\
& \alpha_{\tau \theta}=-(1-v) v_{\tau} v_{\theta}-(1-\alpha)(1-v)\left(v_{\tau}\left[\zeta_{\theta}-(1-\alpha) v_{\theta}\right]+v_{\theta}\left[\zeta_{\tau}-(1-\alpha) v_{\tau}\right]\right) \\
& -(1-v) \zeta_{\tau} \zeta_{\theta}-(1-v)(1-\alpha) v_{\tau}\left[1+(1-\alpha) v_{\theta}\right]-(1-v)\left(\zeta_{\tau}-(1-\alpha) v_{g} \tau\right) \\
& +(1-v)\left[1+(1-\alpha) v_{\theta}\right] \zeta_{\tau}+(1-v)(1-\alpha) \nu_{\tau} \zeta_{\theta}-\chi(1+\phi) \eta_{\tau} \eta_{\theta}-(1+\phi) \frac{\chi^{2}}{1-\chi} \eta_{\theta} \eta_{\tau} \\
& \alpha_{y, t}^{i}=a_{t}^{i}-(1-v) v_{y} a_{t}^{i}-(1-\alpha)(1-v)\left[\zeta_{y}-(1-\alpha) v_{y}\right] a_{t}^{i} \\
& \alpha_{g, t}^{i}=-(1-v) v_{g} a_{t}^{i}-(1-\alpha)(1-v)\left[\zeta_{g}-(1-\alpha) v_{g}\right] a_{t}^{i}-v a_{t}^{i} \\
& \alpha_{\tau, t}^{i}=-(1-v) v_{\tau} a_{t}^{i}-(1-\alpha)(1-v)\left[\zeta_{\tau}-(1-\alpha) \nu_{\tau}\right] a_{t}^{i} \\
& \alpha_{\theta, t}^{i}=-(1-v) v_{\theta} a_{t}^{i}-(1-\alpha)(1-v)\left[\zeta_{\theta}-(1-\alpha) v_{\theta}\right] a_{t}^{i} \text {. }
\end{aligned}
$$

It turns out that we generally have $\alpha_{y, t}^{i}=\alpha_{g, t}^{i}=\alpha_{\tau, t}^{i}=\alpha_{\theta, t}^{i}=0$ for all $t$ and $i$.

\section{D.3.6 Loss Function}

We will find it more convenient later on to work with the loss function instead of the welfare function. The former is the negative of the latter. Moreover, as in the body of the paper, we will be interested in the loss function in gaps from its natural counterpart. Note in (161) that for the loss function under the natural allocation, all terms drop out except for $Z^{i}+\hat{Z}_{t}^{*}+\tilde{Z}_{t}^{i}$. Therefore, we have the following 
loss function in gaps from the natural

$$
\begin{aligned}
& \mathbb{L}=-(U-\tilde{U}) \\
& =\int_{0}^{\infty} \int_{0}^{1} e^{-\rho t}\left[\alpha_{\pi}\left(\hat{\bar{\pi}}_{H, t}^{i}\right)^{2}-\alpha_{y y}\left(\hat{\bar{y}}_{t}^{i}\right)^{2}-\alpha_{\theta \theta}\left(\hat{\bar{\theta}}_{t}^{i}\right)^{2}-\alpha_{\tau \tau}\left(\hat{\bar{\tau}}_{t}^{i, r}\right)^{2}-\alpha_{g g}\left(\hat{\bar{g}}_{t}^{i}\right)^{2}\right. \\
& \left.-\alpha_{y \theta} \hat{\bar{y}}_{t}^{i} \hat{\bar{\theta}}_{t}^{i}-\alpha_{y g} \hat{\bar{y}}_{t}^{i} \hat{\bar{g}}_{t}^{i}-\alpha_{y \tau} \hat{\bar{y}}_{t}^{i} \hat{\bar{\tau}}_{t}^{i, r}-\alpha_{g \theta} \hat{\bar{g}}_{t}^{i} \hat{\bar{\theta}}_{t}^{i}-\alpha_{g \tau} \hat{\bar{g}}_{t}^{i} \hat{\bar{\tau}}_{t}^{i, r}-\alpha_{\tau \theta} \hat{\bar{\tau}}_{t}^{i, r} \hat{\bar{\theta}}_{t}^{i}\right] d i d t \\
& \equiv \int_{0}^{\infty} \int_{0}^{1} e^{-\rho t}\left[\alpha_{\pi}\left(\hat{\bar{\pi}}_{H, t}^{i}\right)^{2}-\hat{U}_{t}^{i}\right] d i d t
\end{aligned}
$$

\section{D.3.7 General Planning Problem}

In summary, in this section we have derived all elements that comprise the general planning problem for country $i$, featuring all three policy instruments. Assembling them now, we can write the fully general country-i planning problem as follows:

$$
\min _{\left\{\hat{\theta}_{t}^{i}, \hat{s}_{\hat{t}}^{i}, \hat{\tau}_{t}^{, r}\right\}_{t \geq 0}} \int_{0}^{\infty} e^{-\rho t}\left[\alpha_{\pi}\left(\hat{\bar{\pi}}_{H, t}^{i}\right)^{2}-\hat{U}_{t}^{i}\right] d t
$$

subject to

$$
\begin{aligned}
\Lambda_{y} \dot{\overline{\hat{y}}}_{t}^{i} & =-\Lambda_{s}\left(\hat{\bar{\pi}}_{H, t}^{i}+\dot{\tilde{s}}_{t}^{i}\right)+\Lambda_{g} \dot{\hat{\bar{g}}}_{t}^{i}+\Lambda_{\theta} \dot{\hat{\bar{\theta}}}_{t}^{i}+\Lambda_{\tau} \dot{\hat{\bar{\tau}}}_{t}^{i, r} \\
\dot{\hat{\bar{\pi}}}_{H, t}^{i} & =\rho \hat{\bar{\pi}}_{H, t}^{i}-\kappa_{y} \hat{\bar{y}}_{t}^{i}-\kappa_{\theta} \hat{\bar{\theta}}_{t}^{i}-\kappa_{\tau} \hat{\bar{\tau}}_{t}^{i, r}-\kappa_{g} \hat{\bar{g}}_{t}^{i} \\
\Lambda_{y} \hat{\bar{y}}_{0}^{i} & =-\Lambda_{s} \tilde{s}_{0}^{i}+\Lambda_{g} \hat{\bar{g}}_{0}^{i}+\Lambda_{\theta} \hat{\bar{\theta}}_{0}^{i}+\Lambda_{\tau} \hat{\bar{\tau}}_{0}^{i_{i}, r} \\
0 & =\int_{0}^{\infty} e^{-\rho t}\left[\Gamma_{y} \hat{\bar{y}}_{t}^{i}+\Gamma_{\tau} \hat{\bar{\tau}}_{t}^{i, r}+\Gamma_{\theta} \hat{\bar{\theta}}_{t}^{i}+\Gamma_{g} \hat{\bar{g}}_{t}^{i}\right] d t .
\end{aligned}
$$

We note again that the coordinated union-wide planning problem coincides with the disaggregated problem, where the social planner solves the above optimal control problem for each country $i \in[0,1]$ separately. This follows from the analogous result derived in the main body of the paper. In the remainder of this appendix, we will therefore only consider the disaggregated planning problem of country $i$ under an idiosyncratic productivity shock.

The constraints in this planning problem are the relevant implementability conditions. All other equilibrium conditions can be used to simply back out the equilibrium variables that are not featured in the planning problem.

\section{D.4 Optimal Transfers}

We now consider the problem where the social planner only has access to ex-post transfers, as in body of the paper. In particular, we have $\hat{\theta}_{t}^{i}=\hat{\bar{\theta}}^{i}$ for all $t$ and $\hat{\bar{g}}_{t}^{i}=0$. 
Country $i$ 's planning problem can then be written as

$$
\min _{\hat{\theta}^{i},\left\{\hat{\tau}_{t}^{i, r}\right\}_{t \geq 0}} \int_{0}^{\infty} e^{-\rho t}\left[\alpha_{\pi}\left(\hat{\bar{\tau}}_{H, t}^{i}\right)^{2}-\left.\hat{U}_{t}^{i}\right|_{\hat{\theta}_{t}^{i}=\hat{\theta}^{i}, \hat{\delta}_{t}^{i}=0}\right] d t
$$

subject to

$$
\begin{aligned}
\dot{\hat{y}}_{t}^{i} & =-D^{\prime}\left(\hat{\bar{\pi}}_{H, t}^{i}+\dot{\tilde{s}}_{t}^{i}\right)+D \hat{\bar{v}}_{t}^{i} \\
\dot{\hat{\bar{\tau}}}_{t}^{i, r} & =\hat{\bar{v}}_{t}^{i} \\
\dot{\vec{\pi}}_{H, t}^{i} & =\rho \hat{\bar{\pi}}_{H, t}^{i}-\kappa_{y} \hat{\bar{y}}_{t}^{i}-\kappa_{\theta} \hat{\theta}^{i}-\kappa_{\tau} \hat{\bar{\tau}}_{t}^{i, r}
\end{aligned}
$$

where $D^{\prime}=\frac{\Lambda_{s}}{\Lambda_{y}}$ and $D=\frac{\Lambda_{\tau}}{\Lambda_{y}}$, and also subject to the intial condition

$$
\Lambda_{y} \hat{\bar{y}}_{0}^{i}=-\Lambda_{s} \tilde{s}_{0}^{i}+\Lambda_{\theta} \hat{\theta}^{i}+\Lambda_{\tau} \hat{\bar{\tau}}_{0}^{i, r}
$$

which will be used to ensure the stability of the dynamical system, and the budget constraint

$$
0=\int_{0}^{\infty} e^{-\rho t}\left[\Gamma_{y} \hat{\bar{y}}_{t}^{i}+\Gamma_{\tau} \hat{\bar{\tau}}_{t}^{i, r}+\Gamma_{\theta} \hat{\theta}^{i}\right] d t
$$

which will be used to solve for $\hat{\theta}^{i}$. In particular, our strategy will be to solve the optimal allocation and policy intervention as a function of $\hat{\theta}^{i}$ analytically, and then numerically solve for the optimal $\hat{\theta}^{i}$ in a second stage.

Also note that we have replaced $\dot{\hat{\tau}}_{t}^{i, r}$ in the (IS) equation with another variable. This is necessary to bring the planning problem into the form of a standard optimal control problem. Finally, we will integrate the initial condition and the budget constraint, an isoperimetric constraint in this case, into the optimal control problem using Lagrange multipliers. In particular, let

- $x_{t}^{i}=\left\{\hat{\bar{\pi}}_{H, t}^{i}, \hat{\bar{y}}_{t}^{i}, \hat{\bar{\tau}}_{t}^{i, r}\right\}$ denote the vector of state variables,

- $u_{t}^{i}=\left\{\hat{v}_{t}^{i}\right\}$ denote the vector of control variables, and

- $\mu_{t}^{i}=\left\{\mu_{\pi, t}^{i}, \mu_{y, t}^{i}, \mu_{\tau, t}^{i}\right\}$ denote the vector of costates.

Then we can summarize country i's optimal control problem with its current-value Hamiltonian, which is given by

$$
\begin{aligned}
H\left(x_{t}^{i}, u_{t}^{i}, \mu_{t}^{i}\right)= & \alpha_{\pi}\left(\pi_{H, t}^{i}\right)^{2}-\left.\hat{U}_{t}^{i}\right|_{\hat{\theta}_{t}^{i}=\hat{\theta}^{i}, \hat{g}_{t}^{i}=0} \\
& +\lambda\left[\Gamma_{y} \hat{\bar{y}}_{t}^{i}+\Gamma_{\tau} \hat{\bar{\tau}}_{t}^{i, r}+\Gamma_{\theta} \hat{\bar{\theta}}^{i}\right] \\
& +\Delta\left[\Lambda_{y} \hat{\bar{y}}_{0}^{i}+\Lambda_{s} \tilde{s}_{0}^{i}-\Lambda_{\theta} \hat{\theta}^{i}-\Lambda_{\tau} \hat{\bar{\tau}}_{0}^{i, r}\right] \\
& +\mu_{y, t}^{i}\left[D \hat{\bar{v}}_{t}^{i}-D^{\prime}\left(\hat{\bar{\tau}}_{H, t}^{i}+\dot{\tilde{s}}_{t}^{i}\right)\right]
\end{aligned}
$$




$$
\begin{aligned}
& +\mu_{\tau, t}^{i} \hat{\bar{v}}_{t}^{i} \\
& +\mu_{\pi, t}^{i}\left[\rho \hat{\bar{\pi}}_{H, t}^{i}-\kappa_{y} \hat{\bar{y}}_{t}^{i}-\kappa_{\theta} \hat{\bar{\theta}}^{i}-\kappa_{\tau} \hat{\bar{\tau}}_{t}^{i, r}\right] .
\end{aligned}
$$

\section{D.4.1 Optimality Conditions}

Since we have transformed the planning problem into a standard optimal control problem, the optimality conditions are given by

$[(1)]$

i. $\partial_{u} H_{t}^{i}=0$ (Optimality)

ii. $\rho \mu_{x, t}^{i}-\dot{\mu}_{x, t}^{i}=\partial_{x} H_{t}^{i}$ (Multiplier)

iii. $\dot{x}_{t}^{i}=\partial_{\mu_{x}} H_{t}^{i}$ (State equations)

iv. $\mu_{x, 0}^{i}=\partial_{x} H_{0}^{i}$ (Initial conditions),

where we abuse notation slightly to let $x$ and $u$ stand in for each element of the respective vectors. $\partial_{x}$ denotes the partial derivative with respect to $x$. This yields the following nine first-order conditions:

Optimality:

$$
D \mu_{y, t}^{i}+\mu_{\tau, t}^{i}=0 .
$$

\section{Multiplier:}

$$
\begin{aligned}
\dot{\mu}_{\pi, t}^{i} & =D^{\prime} \mu_{y, t}^{i}-2 \alpha_{\pi} \hat{\bar{\pi}}_{H, t}^{i} \\
\rho \mu_{y, t}^{i}-\dot{\mu}_{y, t}^{i} & =\lambda \Gamma_{y}-\kappa_{y} \mu_{\pi, t}^{i}-2 \alpha_{y y} \hat{\bar{y}}_{t}^{i}-\alpha_{y \theta} \hat{\bar{\theta}}^{i}-\alpha_{y \tau} \hat{\bar{\tau}}_{t}^{i, r} \\
\rho \mu_{\tau, t}^{i}-\dot{\mu}_{\tau, t}^{i} & =\lambda \Gamma_{\tau}-\kappa_{\tau} \mu_{\pi, t}^{i}-2 \alpha_{\tau \tau} \hat{\bar{\tau}}_{t}^{i, r}-\alpha_{\tau \theta} \hat{\bar{\theta}}^{i}-\alpha_{y \tau} \hat{\bar{y}}_{t}^{i}
\end{aligned}
$$

State:

$$
\begin{aligned}
\dot{\hat{y}}_{t}^{i} & =-D^{\prime}\left(\hat{\bar{\pi}}_{H, t}^{i}+\dot{\tilde{s}}_{t}^{i}\right)+D \hat{\bar{v}}_{t}^{i} \\
\dot{\hat{\bar{\tau}}}_{t}^{i, r} & =\hat{v}_{t}^{i} \\
\dot{\hat{\pi}}_{H, t}^{i} & =\rho \hat{\bar{\pi}}_{H, t}^{i}-\kappa_{y} \hat{\bar{y}}_{t}^{i}-\kappa_{\theta} \hat{\bar{\theta}}^{i}-\kappa_{\tau} \hat{\bar{\tau}}_{t}^{i, r}
\end{aligned}
$$

\section{Initial conditions:}

$$
\begin{aligned}
\mu_{\pi, 0}^{i} & =0 \\
\mu_{y, 0}^{i} & =\Delta \Lambda_{y} \\
\mu_{\tau, 0}^{i} & =-\Delta \Lambda_{\tau} .
\end{aligned}
$$


We will now reduce the size of the above dynamical system by solving out for $\mu_{\pi, t}^{i}$ and $\mu_{\tau, t}^{i}$. Using equations (168), (170) and (171), we can write

$$
\left(\kappa_{\tau}+D \kappa_{y}\right) \mu_{\pi, t}^{i}=\left(\Gamma_{y} D+\Gamma_{\tau}\right) \lambda-\left(2 \alpha_{\tau \tau}+D \alpha_{y \tau}\right) \hat{\bar{\tau}}_{t}^{i, r}-\left(2 D \alpha_{y y}+\alpha_{y \tau}\right) \hat{\bar{y}}_{t}^{i}-\left(\alpha_{\tau \theta}+D \alpha_{y \theta}\right) \hat{\bar{\theta}}^{i}
$$

Taking the derivative with respect to time and using (169), we have

$$
\left(\kappa_{\tau}+D \kappa_{y}\right)\left(D^{\prime} \mu_{y, t}^{i}-2 \alpha_{\pi} \hat{\bar{\pi}}_{H, t}^{i}\right)+\left(2 \alpha_{\tau \tau}+D \alpha_{y \tau}\right) \dot{\overline{\hat{\tau}}}_{t}^{i, r}+\left(2 D \alpha_{y y}+\alpha_{y \tau}\right) \dot{\bar{y}}_{t}^{i}=0,
$$

from which we can solve for the rate of change of transfers using the (IS) equation:

$$
\dot{\hat{\bar{\tau}}}_{t}^{i, r}=-\frac{\left(\kappa_{\tau}+D \kappa_{y}\right)\left(D^{\prime} \mu_{y, t}^{i}-2 \alpha_{\pi} \hat{\bar{\pi}}_{H, t}^{i}\right)-D^{\prime}\left(2 D \alpha_{y y}+\alpha_{y \tau}\right)\left(\hat{\bar{\pi}}_{H, t}^{i}+\dot{\tilde{s}}_{t}^{i}\right)}{2 \alpha_{\tau \tau}+D \alpha_{y \tau}+D\left(2 D \alpha_{y y}+\alpha_{y \tau}\right)}
$$

Using the (IS) equation again, we have

$$
\dot{\hat{y}}_{t}^{i}=-D^{\prime}\left(\hat{\bar{\pi}}_{H, t}^{i}+\dot{\tilde{s}}_{t}^{i}\right)-D \frac{\left(\kappa_{\tau}+D \kappa_{y}\right)\left(D^{\prime} \mu_{y, t}^{i}-2 \alpha_{\pi} \hat{\bar{\pi}}_{H, t}^{i}\right)-D^{\prime}\left(2 D \alpha_{y y}+\alpha_{y \tau}\right)\left(\hat{\bar{\pi}}_{H, t}^{i}+\dot{\tilde{s}}_{t}^{i}\right)}{2 \alpha_{\tau \tau}+D \alpha_{y \tau}+D\left(2 D \alpha_{y y}+\alpha_{y \tau}\right)} .
$$

Finally, using (170) and our solution for $\mu_{\pi, t}^{i}$ above, we find

$$
\dot{\mu}_{y, t}^{i}=\rho \mu_{y, t}^{i}+\lambda \frac{\kappa_{y} \Gamma_{\tau}-\kappa_{\tau} \Gamma_{y}}{\kappa_{\tau}+D \kappa_{y}}-\hat{\bar{\tau}}_{t}^{i, r} \frac{2 \kappa_{y} \alpha_{\tau \tau}-\kappa_{\tau} \alpha_{y \tau}}{\kappa_{\tau}+D \kappa_{y}}-\hat{\bar{y}}_{t}^{i} \frac{\alpha_{y \tau} \kappa_{y}-2 \kappa_{\tau} \alpha_{y y}}{\kappa_{\tau}+D \kappa_{y}}-\hat{\bar{\theta}}^{i} \frac{\kappa_{y} \alpha_{\tau \theta}-\kappa_{\tau} \alpha_{y \theta}}{\kappa_{\tau}+D \kappa_{y}} .
$$

Therefore, we can write the reduced system of optimality conditions as

$$
\begin{aligned}
\dot{\mu}_{y, t}^{i} & =\rho \mu_{y, t}^{i}+K_{\tau} \hat{\bar{\tau}}_{t}^{i, r}+K_{y} \hat{\bar{y}}_{t}^{i}+K_{\theta} \hat{\bar{\theta}}^{i}+K_{\lambda} \lambda \\
\dot{\hat{y}}_{t}^{i} & =J_{\mu_{y}} \mu_{y, t}^{i}+J_{\pi} \hat{\bar{\pi}}_{H, t}^{i}+J_{s} \dot{\tilde{s}}_{t}^{i} \\
\dot{\bar{\tau}}_{t}^{i, r} & =H_{\mu_{y}} \mu_{y, t}^{i}+H_{\pi} \hat{\bar{\pi}}_{H, t}^{i}+H_{s} \dot{\tilde{s}}_{t}^{i} \\
\dot{\overline{\bar{\tau}}}_{H, t}^{i} & =\rho \hat{\bar{\pi}}_{H, t}^{i}-\kappa_{y} \hat{\bar{y}}_{t}^{i}-\kappa_{\theta} \hat{\bar{\theta}}^{i}-\kappa_{\tau} \hat{\bar{\tau}}_{t}^{i, r} .
\end{aligned}
$$

\section{D.4.2 Solving the Dynamic System}

In particular, the optimality conditions yield a system of linear ODEs which, letting $X_{t}^{i}=\left\{\hat{\bar{\pi}}_{H, t}^{i}, \hat{\bar{y}}_{t}^{i}, \hat{\bar{\tau}}_{t}^{i, r}, \mu_{y, t}^{i}\right\}$, we can express as

$$
\dot{X}_{t}^{i}=A X_{t}^{i}+B_{t}^{i}
$$

where

$$
A=\left(\begin{array}{cccc}
\rho & -\kappa_{y} & -\kappa_{\tau} & 0 \\
J_{\pi} & 0 & 0 & J_{\mu_{y}} \\
H_{\pi} & 0 & 0 & H_{\mu_{y}} \\
0 & K_{y} & K_{\tau} & \rho
\end{array}\right), \quad B_{t}^{i}=\left(\begin{array}{c}
-\kappa_{\theta} \hat{\bar{\theta}}^{i} \\
\bar{J}_{t}^{i} \\
\bar{H}_{t}^{i} \\
\bar{K}_{t}^{i}
\end{array}\right), \quad X_{0}^{i}=\left(\begin{array}{c}
\hat{\bar{\tau}}_{H, 0}^{i} \\
\hat{\bar{y}}_{0}^{i} \\
\hat{\bar{\tau}}_{0}^{i, r} \\
\Delta \Lambda_{y}
\end{array}\right),
$$


where $\bar{J}_{t}^{i}=J_{s} \dot{\tilde{s}}_{t}^{i}, \bar{H}_{t}^{i}=H_{s} \dot{\tilde{s}}_{t}^{i}$, and $\bar{K}_{t}^{i}=K_{\theta} \hat{\bar{\theta}}^{i}+K_{\lambda} \lambda$.

The solution to this dynamical system is, as is well known, given by

$$
X_{t}^{i}=e^{A t}\left[X_{0}^{i}+\int_{0}^{t} e^{-A s} B_{s}^{i} d s\right]
$$

which we can rewrite as

$$
X_{t}^{i}=e^{A t}\left[X_{0}^{i}+\int_{0}^{t} e^{-A s}\left(-\kappa_{\theta} \hat{\bar{\theta}}^{i} E_{1}+\bar{J}_{s}^{i} E_{2}+\bar{H}_{s}^{i} E_{3}+\bar{K}_{s}^{i} E_{4} d s\right]\right.
$$

where $E_{i}$ is the $4 \times 1$ zero vector with a 1 in the $i^{\text {th }}$ position. We can write

$$
\begin{aligned}
X_{t}^{i}=e^{A t} & {\left[X_{0}^{i}-\kappa_{\theta} \hat{\bar{\theta}}^{i} \int_{0}^{t} e^{-A s} E_{1} d s+\left(K_{\theta} \hat{\bar{\theta}}^{i}+K_{\lambda} \lambda\right) \int_{0}^{t} e^{-A s} E_{4} d s\right.} \\
& \left.+W_{2} \int_{0}^{t} e^{-A s-\psi s} E_{2} d s+W_{3} \int_{0}^{t} e^{-A s-\psi s} E_{3} d s\right],
\end{aligned}
$$

where $W_{2}=-\psi a_{0}^{i} J_{s}$, and $W_{3}=-\psi a_{0}^{i} H_{s}$.

Assuming that the economy is in the parameter subspace where $A$ is nonsingular, we can use the results $\int_{0}^{t} e^{-A s} d s=A^{-1}\left(I-e^{-A t}\right)$ and $\int_{0}^{t} e^{-(A+\psi I) s} d s=(A+\psi I)^{-1}\left(I-e^{-(A+\psi I) t}\right)$ to solve the integral, so that

$$
\begin{aligned}
X_{t}^{i}=e^{A t} & {\left[X_{0}^{i}-\kappa_{\theta} \hat{\bar{\theta}}^{i} A^{-1}\left(I-e^{-A t}\right) E_{1}+\left(K_{\theta} \hat{\bar{\theta}}^{i}+K_{\lambda} \lambda\right) A^{-1}\left(I-e^{-A t}\right) E_{4}\right.} \\
& \left.+W_{2}(A+\psi I)^{-1}\left(I-e^{-(A+\psi I) t}\right) E_{2}+W_{3}(A+\psi I)^{-1}\left(I-e^{-(A+\psi I) t}\right) E_{3}\right] .
\end{aligned}
$$

From this, we finally arrive at the solution

$$
\begin{aligned}
X_{t}^{i}= & e^{A t}\left[X_{0}^{i}-\kappa_{\theta} \hat{\bar{\theta}}^{i} A^{-1} E_{1}+\left(K_{\theta} \hat{\bar{\theta}}^{i}+K_{\lambda} \lambda\right) A^{-1} E_{4}+(A+\psi I)^{-1}\left(W_{2} E_{2}+W_{3} E_{3}\right)\right] \\
& +\kappa_{\theta} \hat{\bar{\theta}}^{i} A^{-1} E_{1}-\left(K_{\theta} \hat{\bar{\theta}}^{i}+K_{\lambda} \lambda\right) A^{-1} E_{4}-(A+\psi I)^{-1} e^{-\psi t}\left(W_{2} E_{2}+W_{3} E_{3}\right)
\end{aligned}
$$

\section{D.4.3 Stability}

There is a unique solution if and only if $A$ has two negative eigenvalues. For stability, we then need

$$
X_{0}^{i}-\kappa_{\theta} \hat{\bar{\theta}}^{i} A^{-1} E_{1}+\left(K_{\theta} \hat{\theta}^{i}+K_{\lambda} \lambda\right) A^{-1} E_{4}+(A+\psi I)^{-1}\left(W_{2} E_{2}+W_{3} E_{3}\right)
$$

to be in the stable manifold, the subspace of the state space spanned by the eigenvectors associated with the negative eigenvalues. Let $V_{j}, j \in\{1,2\}$, be the eigenvector of $A$ associated with the negative 
eigenvalue $\lambda_{j}$. Then there must be $\alpha_{j} \in \mathbb{C}, j \in\{1,2\}$ such that

$$
\begin{aligned}
0= & \alpha_{1} V_{1}+\alpha_{2} V_{2}+X_{0}^{i}-\kappa_{\theta} \hat{\bar{\theta}}^{i} A^{-1} E_{1}+\left(K_{\theta} \hat{\theta}^{i}+K_{\lambda} \lambda\right) A^{-1} E_{4} \\
& +(A+\psi I)^{-1}\left(W_{2} E_{2}+W_{3} E_{3}\right) .
\end{aligned}
$$

In particular, to guarantee stability we need to solve for the vector $Z=\left(\alpha_{1}, \alpha_{2}, \lambda, \Delta, \hat{\bar{\pi}}_{H, 0}^{i} \hat{\bar{y}}_{0}^{i}, \hat{\bar{\tau}}_{0}^{i, r}\right)^{\prime} \in$ $\mathbb{C}^{7}$. Therefore, we need seven linearly independent conditions involving the desired variables. Equation (185) yields four of these conditions. To see this, left-multiply (185) by $E_{i}^{\prime}$ for each $i \in\{1,2,3,4\}$.

The fifth stability condition will be the initial condition, $\Lambda_{y} \hat{\bar{y}}_{0}^{i}=-\Lambda_{s} \tilde{s}_{0}^{i}+\Lambda_{\tau} \hat{\bar{\tau}}_{0}^{i, r}+\Lambda_{\theta} \hat{\bar{\theta}}^{i}$. The sixth condition we obtain by writing

$$
\begin{aligned}
\left(\kappa_{\tau}+D \kappa_{y}\right) \mu_{\pi, 0}^{i} & =0 \\
& =\left(\Gamma_{y} D+\Gamma_{\tau}\right) \lambda-\left(2 \alpha_{\tau \tau}+D \alpha_{y \tau}\right) \hat{\bar{\tau}}_{0}^{i, r}-\left(2 D \alpha_{y y}+\alpha_{y \tau}\right) \hat{\bar{y}}_{0}^{i}-\left(\alpha_{\tau \theta}+D \alpha_{y \theta}\right) \hat{\bar{\theta}}^{i} .
\end{aligned}
$$

The seventh and final condition we obtain from country $i$ 's budget constraint. In particular, we have

$$
\begin{aligned}
0 & =\int_{0}^{\infty} e^{-\rho t}\left[\Gamma_{y} \hat{\bar{y}}_{t}^{i}+\Gamma_{\tau} \hat{\bar{\tau}}_{t}^{i, r}+\Gamma_{\theta} \hat{\bar{\theta}}^{i}\right] d t \\
& =\frac{\Gamma_{\theta}}{\rho} \hat{\theta}^{i}+\int_{0}^{\infty} e^{-\rho t}\left(\Gamma_{y} E_{2}+\Gamma_{\tau} E_{3}\right)^{\prime} X_{t}^{i} d t .
\end{aligned}
$$

We can write

$$
\begin{aligned}
X_{t}^{i} & =e^{A t}\left(-\alpha_{1} V_{1}-\alpha_{2} V_{2}\right)+\kappa_{\theta} \hat{\bar{\theta}}^{i} A^{-1} E_{1}-\left(K_{\theta} \hat{\theta}^{i}+K_{\lambda} \lambda\right) A^{-1} E_{4}-(A+\psi I)^{-1} e^{-\psi t}\left(W_{2} E_{2}+W_{3} E_{3}\right) \\
& =-\alpha_{1} e^{\lambda_{1} t} V_{1}-\alpha_{2} e^{\lambda_{2} t} V_{2}+\kappa_{\theta} \hat{\theta}^{i} A^{-1} E_{1}-\left(K_{\theta} \hat{\theta}^{i}+K_{\lambda} \lambda\right) A^{-1} E_{4}-(A+\psi I)^{-1} e^{-\psi t}\left(W_{2} E_{2}+W_{3} E_{3}\right) .
\end{aligned}
$$

Plugging this in and solving the integrals, we obtain our final stability condition

$$
\begin{aligned}
0= & \frac{\Gamma_{\theta}}{\rho} \hat{\theta}^{i}+\frac{\alpha_{1}}{\lambda_{1}-\rho}\left(\Gamma_{y} E_{2}+\Gamma_{\tau} E_{3}\right)^{\prime} V_{1}+\frac{\alpha_{2}}{\lambda_{2}-\rho}\left(\Gamma_{y} E_{2}+\Gamma_{\tau} E_{3}\right)^{\prime} V_{2} \\
& +\frac{\kappa_{\theta} \hat{\theta}^{i}}{\rho}\left(\Gamma_{y} E_{2}+\Gamma_{\tau} E_{3}\right)^{\prime} A^{-1} E_{1}-\frac{K_{\theta} \hat{\theta}^{i}+K_{\lambda} \lambda}{\rho}\left(\Gamma_{y} E_{2}+\Gamma_{\tau} E_{3}\right)^{\prime} A^{-1} E_{4} \\
& -\frac{1}{\rho+\psi}\left(\Gamma_{y} E_{2}+\Gamma_{\tau} E_{3}\right)^{\prime}(A+\psi I)^{-1}\left[W_{2} E_{2}+W_{3} E_{3}\right] .
\end{aligned}
$$


Putting everything together, we can write $M Z=N$, where

$$
M=\left(\begin{array}{ccccccc}
E_{1}^{\prime} V_{1} & E_{1}^{\prime} V_{2} & K_{\lambda} E_{1}^{\prime} A^{-1} E_{4} & 0 & 1 & 0 & 0 \\
E_{2}^{\prime} V_{1} & E_{2}^{\prime} V_{2} & K_{\lambda} E_{2}^{\prime} A^{-1} E_{4} & 0 & 0 & 1 & 0 \\
E_{3}^{\prime} V_{1} & E_{3}^{\prime} V_{2} & K_{\lambda} E_{3}^{\prime} A^{-1} E_{4} & 0 & 0 & 0 & 1 \\
E_{4}^{\prime} V_{1} & E_{4}^{\prime} V_{2} & K_{\lambda} E_{4}^{\prime} A^{-1} E_{4} & \Lambda_{y} & 0 & 0 & 0 \\
0 & 0 & 0 & 0 & 0 & \Lambda_{y} & -\Lambda_{\tau} \\
0 & 0 & \Gamma_{\tau}+D \Gamma_{y} & 0 & 0 & -\left(2 \alpha_{y y} D+\alpha_{y \tau}\right) & -\left(2 \alpha_{\tau \tau}+D \alpha_{y \tau}\right) \\
\frac{\left(\Gamma_{y} E_{2}+\Gamma_{\tau} E_{3}\right)^{\prime}}{\lambda_{1}-\rho} V_{1} & \frac{\left(\Gamma_{y} E_{2}+\Gamma_{\tau} E_{3}\right)^{\prime}}{\lambda_{2}-\rho} V_{2} & -\frac{K_{\lambda}}{\rho}\left(\Gamma_{y} E_{2}+\Gamma_{\tau} E_{3}\right)^{\prime} A^{-1} E_{4} & 0 & 0 & 0 & 0
\end{array}\right)
$$

and

$$
N=\left(\begin{array}{c}
-E_{1}^{\prime} W \\
-E_{2}^{\prime W} \\
-E_{3}^{\prime} W \\
-E_{4}^{\prime} W \\
-\Lambda_{s} \tilde{s}_{0}^{i}+\Lambda_{\theta} \hat{\theta}^{i} \\
\left(\alpha_{\tau \theta}+D \alpha_{y \theta}\right)^{i} \\
-\frac{\Gamma_{\theta} \hat{\theta}^{i}}{\rho}-\frac{\kappa_{\theta} \hat{\theta}^{i}}{\rho}\left(\Gamma_{y} E_{2}+\Gamma_{\tau} E_{3}\right)^{\prime} A^{-1} E_{1}+\frac{K_{\theta} \hat{\theta}^{i}}{\rho}\left(\Gamma_{y} E_{2}+\Gamma_{\tau} E_{3}\right)^{\prime} A^{-1} E_{4}+\frac{\left(\Gamma_{y} E_{2}+\Gamma_{\tau} E_{3}\right)^{\prime}}{\rho+\psi}(A+\psi I)^{-1}\left[W_{2} E_{2}+W_{3} E_{3}\right]
\end{array}\right)
$$

where $W=-\kappa_{\theta} \hat{\bar{\theta}}^{i} A^{-1} E_{1}+K_{\theta} \hat{\theta}^{i} A^{-1} E_{4}+(A+\psi I)^{-1}\left[W_{2} E_{2}+W_{3} E_{3}\right]$. Given these matrices, we can compute the vector $Z$ numerically by setting

$$
Z=M^{-1} N
$$

over the parameter subspace on which $M$ is invertible.

\section{D.5 Optimal Capital Controls}

We now consider the case where the social planner only has access to capital controls. That is, $\hat{\hat{g}}_{t}^{i}=0$ for all $t$ and $N F A_{0}^{i}=0$. The planner's choice of capital controls will be encoded in a time-varying $\hat{\theta}_{t}^{i}$.

This directly implies that $N \hat{\bar{F}} A_{0}^{i}=0$ in the absence of transfers. Furthermore, since we assume that the government does not engage in additional redistribution, we have $\tau_{t}^{i, r}=-\left(S_{t}^{i}\right)^{-\alpha} G_{t}^{i}$. Similarly, $\tau_{t}^{i, r}=\tau_{t}^{i, o}$ because the government does not discriminate between different types of agents. Hence, we have

$$
\hat{\bar{\tau}}_{t}^{i, r}=\alpha v \hat{\bar{s}}_{t}^{i}
$$

Plugging in for $\hat{\bar{s}}_{t}^{i}=v_{y} \hat{\bar{y}}_{t}^{i}+v_{g} \hat{\bar{g}}_{t}^{i}+v_{\tau} \hat{\bar{\tau}}_{t}^{i}+v_{\theta} \hat{\theta}_{t}^{i}$, with $\hat{\bar{g}}_{t}^{i}=0$, we find that

$$
\begin{aligned}
& \hat{\bar{\tau}}_{t}^{i, r}=\frac{\alpha v}{1-\alpha v v_{\tau}}\left(v_{y} \hat{\bar{y}}_{t}^{i}+v_{\theta} \hat{\bar{\theta}}_{t}^{i}\right) \\
& \dot{\hat{\bar{\tau}}}_{t}^{i, r}=\frac{\alpha v}{1-\alpha v v_{\tau}}\left(v_{y} \dot{\hat{y}}_{t}^{i}+v_{\theta} \dot{\hat{\theta}}_{t}^{i}\right) .
\end{aligned}
$$


IS equation. This allows us to rewrite the general (IS) equation for this particular allocation as

$$
\Lambda_{y} \dot{\hat{y}}_{t}^{i}=-\Lambda_{s}\left(\hat{\bar{\pi}}_{H, t}^{i}+\dot{\tilde{s}}_{t}^{i}\right)+\frac{\alpha v v_{y}}{1-\alpha v v_{\tau}} \Lambda_{\tau} \dot{\hat{\bar{y}}}_{t}^{i}+\left(\Lambda_{\theta}+\frac{\alpha v v_{\theta}}{1-\alpha v v_{\tau}} \Lambda_{\tau}\right) \dot{\hat{\theta}}_{t}^{i},
$$

or, more conveniently,

$$
\dot{\bar{y}}_{t}^{i}=-G^{\prime}\left(\hat{\bar{\pi}}_{H, t}^{i}+\dot{\tilde{s}}_{t}^{i}\right)+G \dot{\hat{\bar{\theta}}}_{t}^{i}
$$

where

$$
\begin{aligned}
G & =\frac{\Lambda_{\theta}+\frac{\alpha v v_{\theta}}{1-\alpha v v_{\tau}} \Lambda_{\tau}}{\Lambda_{y}-\frac{\alpha v v_{y}}{1-\alpha v v_{\tau}} \Lambda_{\tau}} \\
G^{\prime} & =\frac{\Lambda_{s}}{\Lambda_{y}-\frac{\alpha v v_{y}}{1-\alpha v v_{\tau}} \Lambda_{\tau}} .
\end{aligned}
$$

NKPC. Similarly, we can rewrite the Phillips Curve noting that we now have

$$
\dot{\bar{\pi}}_{H, t}^{i}=\rho \hat{\bar{\pi}}_{H, t}^{i}-\kappa_{y} \hat{\bar{y}}_{t}^{i}-\kappa_{\theta} \hat{\bar{\theta}}_{t}^{i}-\frac{\alpha v}{1-\alpha v v_{\tau}} \kappa_{\tau}\left(v_{y} \hat{\bar{y}}_{t}^{i}+v_{\theta} \hat{\bar{\theta}}_{t}^{i}\right),
$$

or, more conveniently,

$$
\dot{\bar{\pi}}_{H, t}^{i}=\rho \hat{\bar{\pi}}_{H, t}^{i}-\hat{\kappa}_{y} \hat{\bar{y}}_{t}^{i}-\hat{\kappa}_{\theta} \hat{\bar{\theta}}_{t}^{i}
$$

where

$$
\begin{aligned}
& \hat{\kappa}_{y}=\kappa_{y}+\frac{\alpha v}{1-\alpha v v_{\tau}} \kappa_{\tau} v_{y} \\
& \hat{\kappa}_{\theta}=\kappa_{\theta}+\frac{\alpha v}{1-\alpha v v_{\tau}} \kappa_{\tau} v_{\theta} .
\end{aligned}
$$

Initial condition. Following similar conversions relative to the general specification presented in the last section, we find that

$$
\hat{\bar{y}}_{0}^{i}=-\bar{G}^{\prime} \tilde{s}_{0}^{i}+\bar{G} \hat{\bar{\theta}}_{0}^{i}
$$

for

$$
\begin{aligned}
\bar{G} & =\frac{\Lambda_{\theta}}{\Lambda_{y}} \\
\bar{G}^{\prime} & =\frac{\Lambda_{s}+\alpha v \Lambda_{\tau}}{\Lambda_{y}} .
\end{aligned}
$$

NFA. Finally, we record here the simplified country-i budget constraint when only capital controls are used. We have

$$
0=\int_{0}^{\infty} e^{-\rho t}\left(\hat{\Gamma}_{y} \hat{\bar{y}}_{t}^{i}+\hat{\Gamma}_{\theta} \hat{\bar{\theta}}_{t}^{i}\right) d t
$$


where

$$
\begin{aligned}
& \hat{\Gamma}_{y}=\Gamma_{y}+\frac{\alpha v}{1-\alpha v v_{\tau}} v_{y} \Gamma_{\tau} \\
& \hat{\Gamma}_{\theta}=\Gamma_{\theta}+\frac{\alpha v}{1-\alpha v v_{\tau}} v_{\theta} \Gamma_{\tau} .
\end{aligned}
$$

We note at this point that $\hat{\Gamma}_{y}=0$ over the entire parameter space. We will continue to carry the term around in this section, but we want to highlight the important implication of this result: informally, present-value capital controls must always average to zero over time. Formally, of course, we must have

$$
0=\int_{0}^{\infty} e^{-\rho t} \hat{\Gamma}_{\theta} \hat{\bar{\theta}}_{t}^{i} d t=\int_{0}^{\infty} e^{-\rho t} \hat{\bar{\theta}}_{t}^{i} d t
$$

Disaggregated control problem. We are now in a position to transform country-i's disaggregated planning problem to the form of a standard optimal control problem. In particular, the planning problem is given by

$$
\min _{\left\{\hat{\theta}_{t}^{i}\right\}_{t \geq 0}} \int_{0}^{\infty} e^{-\rho t}\left[\alpha_{\pi}\left(\hat{\bar{\pi}}_{H, t}^{i}\right)^{2}-\left.\hat{U}_{t}^{i}\right|_{\hat{\bar{g}}_{t}^{i}=0, \hat{\tau}_{t}^{i, r}=\alpha v \hat{\bar{s}}_{t}^{i}}\right] d t
$$

subject to

$$
\begin{aligned}
\dot{\hat{\bar{y}}}_{t}^{i} & =-G^{\prime}\left(\hat{\bar{\pi}}_{H, t}^{i}+\dot{\tilde{s}}_{t}^{i}\right)+G \dot{\overline{\hat{\theta}}}_{t}^{i} \\
\dot{\bar{\pi}}_{H, t}^{i} & =\rho \hat{\bar{\pi}}_{H, t}^{i}-\hat{\kappa}_{y} \hat{\bar{y}}_{t}^{i}-\hat{\kappa}_{\theta} \hat{\bar{\theta}}_{t}^{i} \\
\hat{\bar{y}}_{0}^{i} & =-\bar{G}^{\prime} \tilde{s}_{0}^{i}+\bar{G}^{i} \\
0 & =\int_{0}^{\infty} e^{-\rho t}\left(\hat{\bar{\Gamma}}_{y} \hat{\bar{y}}_{t}^{i}+\hat{\Gamma}_{\theta} \hat{\bar{\theta}}_{t}^{i}\right) d t .
\end{aligned}
$$

As in the previous section for optimal transfers, the conversion from planning two optimal control problem requires to important steps. First, we include the initial condition and the isoperimetric budget constraint in the Hamiltonian using Lagrange multipliers. Second, we have to substitute out the time derivative $\dot{\hat{\theta}}_{t}^{i}$ in the (IS) equation and replace it with placeholder $\hat{\bar{v}}_{t}^{i}$. We record country $i^{\prime}$ s disaggregated optimal control problem in the form of the following Hamiltonian:

$$
\begin{aligned}
H_{t}^{i}\left(x_{t}^{i}, u_{t}^{i}, \mu_{t}^{i}\right)= & \alpha_{\pi}\left(\pi_{H, t}^{i}\right)^{2}-\left.\hat{\bar{U}}_{t}^{i}\right|_{\hat{\bar{g}}_{t}^{i}=0, \hat{\bar{\tau}}_{t}^{i, r}=\alpha v \hat{\bar{s}}_{t}^{i}} \\
& +\lambda\left[\hat{\Gamma}_{y} \hat{\bar{y}}_{t}^{i}+\hat{\Gamma}_{\theta} \hat{\bar{\theta}}_{t}^{i}\right] \\
& +\Delta\left[\hat{\bar{y}}_{0}^{i}+\bar{G}^{\prime} \tilde{s}_{0}^{i}-\bar{G} \hat{\bar{\theta}}_{0}^{i}\right] \\
& +\mu_{y, t}^{i}\left[G \hat{\bar{v}}_{t}^{i}-G^{\prime}\left(\hat{\bar{\pi}}_{H, t}^{i}+\dot{\tilde{s}}_{t}^{i}\right)\right] \\
& +\mu_{\theta, t}^{i} \hat{\bar{v}}_{t}^{i}
\end{aligned}
$$




$$
+\mu_{\pi, t}^{i}\left[\rho \hat{\bar{\pi}}_{H, t}^{i}-\hat{\kappa}_{y} \hat{\bar{y}}_{t}^{i}-\hat{\kappa}_{\theta} \hat{\bar{\theta}}_{t}^{i}\right] .
$$

As before, $x_{t}^{i}$ describes the vector of state variables, $u_{t}^{i}$ the vector of control variables, and $\mu_{t}^{i}$ the vector of costates.

Loss function. Finally, to work with the above Hamiltonian we have to record the precise form that the loss function takes when the planner can only use capital controls. It is straightforward to verify that we have

$$
\left.\hat{\bar{U}}_{t}^{i}\right|_{\hat{g}_{t}^{i}=0, \hat{\bar{\tau}}_{t}^{i, r}=\alpha v \hat{s}_{t}^{i}}=\hat{\alpha}_{y y}\left(\hat{\bar{y}}_{t}^{i}\right)^{2}+\hat{\alpha}_{\theta \theta}\left(\hat{\bar{\theta}}_{t}^{i}\right)^{2}+\hat{\alpha}_{y \theta} \hat{\bar{y}}_{t}^{i} \hat{\bar{\theta}}_{t}^{i}
$$

where

$$
\begin{aligned}
& \hat{\alpha}_{y y}=\alpha_{y y}+\left(\frac{\alpha v}{1-\alpha v v_{\tau}}\right)^{2} v_{y}^{2} \alpha_{\tau \tau}+\frac{\alpha v}{1-\alpha v v_{\tau}} v_{y} \alpha_{y \tau} \\
& \hat{\alpha}_{\theta \theta}=\alpha_{\theta \theta}+\left(\frac{\alpha v}{1-\alpha v v_{\tau}}\right)^{2} v_{\theta}^{2} \alpha_{\tau \tau}+\frac{\alpha v}{1-\alpha v v_{\tau}} v_{\theta} \alpha_{\tau \theta} \\
& \hat{\alpha}_{y \theta}=2 \alpha_{\tau \tau}\left(\frac{\alpha v}{1-\alpha v v_{\tau}}\right)^{2} v_{y} v_{\tau}+\alpha_{y \theta}+\frac{\alpha v}{1-\alpha v v_{\tau}} v_{\theta} \alpha_{y \tau}+\frac{\alpha v}{1-\alpha v v_{\tau}} v_{y} \alpha_{\tau \theta} .
\end{aligned}
$$

\section{D.5.1 Optimality Conditions}

The Hamiltonian is associated with the following first-order conditions.

\section{Optimality:}

$$
G \mu_{y, t}^{i}+\mu_{\tau, t}^{i}=0
$$

\section{Multiplier:}

$$
\begin{aligned}
\dot{\mu}_{\pi, t}^{i} & =G^{\prime} \mu_{y, t}^{i}-2 \alpha_{\pi} \hat{\bar{\pi}}_{H, t}^{i} \\
\rho \mu_{y, t}^{i}-\dot{\mu}_{y, t}^{i} & =\lambda \hat{\Gamma}_{y}-\hat{\kappa}_{y} \mu_{\pi, t}^{i}-2 \hat{\alpha}_{y y} \hat{\bar{y}}_{t}^{i}-\hat{\alpha}_{y \theta} \hat{\bar{\theta}}_{t}^{i} \\
\rho \mu_{\theta, t}^{i}-\dot{\mu}_{\theta, t}^{i} & =\lambda \hat{\Gamma}_{\theta}-\hat{\kappa}_{\theta} \mu_{\pi, t}^{i}-2 \hat{\alpha}_{\theta \theta} \hat{\bar{\theta}}_{t}^{i}-\hat{\alpha}_{y \theta} \hat{\bar{y}}_{t}^{i}
\end{aligned}
$$

\section{State:}

$$
\begin{aligned}
\dot{\hat{\bar{y}}}_{t}^{i} & =-G^{\prime}\left(\hat{\bar{\pi}}_{H, t}^{i}+\dot{\tilde{s}}_{t}^{i}\right)+G \hat{\bar{v}}_{t}^{i} \\
\dot{\overline{\hat{\theta}}}_{t}^{i} & =\hat{\bar{v}}_{t}^{i} \\
\dot{\overline{\bar{\pi}}}_{H, t}^{i} & =\rho \hat{\bar{\pi}}_{H, t}^{i}-\hat{\kappa}_{y} \hat{\bar{y}}_{t}^{i}-\hat{\kappa}_{\theta} \hat{\bar{\theta}}_{t}^{i}
\end{aligned}
$$

\section{Initial conditions:}

$$
\begin{aligned}
& \mu_{\pi, 0}^{i}=0 \\
& \mu_{y, 0}^{i}=\Delta
\end{aligned}
$$




$$
\mu_{\theta, 0}^{i}=-\Delta \bar{G}
$$

We will now reduce the size of the above dynamical system by solving out for $\mu_{\pi, t}^{i}$ and $\mu_{\theta, t}^{i}$. Using equations (201), (203) and (204), we can write

$$
\left(\hat{\kappa}_{\theta}+G \hat{\kappa}_{y}\right) \mu_{\pi, t}^{i}=\left(\hat{\Gamma}_{y} G+\hat{\Gamma}_{\theta}\right) \lambda-\left(2 \hat{\alpha}_{\theta \theta}+G \hat{\alpha}_{y \theta}\right) \hat{\bar{\theta}}_{t}^{i}-\left(2 G \hat{\alpha}_{y y}+\hat{\alpha}_{y \theta}\right) \hat{y}_{t}^{i} .
$$

Taking the derivative with respect to time and using (202), we have

$$
\left(\hat{\kappa}_{\theta}+G \hat{\kappa}_{y}\right)\left(G^{\prime} \mu_{y, t}^{i}-2 \alpha_{\pi} \hat{\bar{\pi}}_{H, t}^{i}\right)+\left(2 \hat{\alpha}_{\theta \theta}+G \hat{\alpha}_{y \theta}\right) \dot{\hat{\theta}}_{t}^{i}+\left(2 G \hat{\alpha}_{y y}+\hat{\alpha}_{y \theta}\right) \dot{\hat{y}}_{t}^{i}=0,
$$

from which we can solve for optimal capital controls using the (IS) equation:

$$
\dot{\hat{\bar{\theta}}}_{t}^{i}=-\frac{\left(\hat{\kappa}_{\theta}+G \hat{\kappa}_{y}\right)\left(G^{\prime} \mu_{y, t}^{i}-2 \alpha_{\pi} \hat{\pi}_{H, t}^{i}\right)-G^{\prime}\left(2 G \hat{\alpha}_{y y}+\hat{\alpha}_{y \theta}\right)\left(\hat{\pi}_{H, t}^{i}+\dot{\tilde{s}}_{t}^{i}\right)}{2 \hat{\alpha}_{\theta \theta}+G \hat{\alpha}_{y \theta}+G\left(2 G \hat{\alpha}_{y y}+\hat{\alpha}_{y \theta}\right)}
$$

Using the (IS) equation again, we have

$$
\dot{\hat{y}}_{t}^{i}=-G^{\prime}\left(\hat{\pi}_{H, t}^{i}+\dot{\tilde{s}}_{t}^{i}\right)-G \frac{\left(\hat{\kappa}_{\theta}+G \hat{\kappa}_{y}\right)\left(G^{\prime} \mu_{y, t}^{i}-2 \alpha_{\pi} \hat{\bar{\pi}}_{H, t}^{i}\right)-G^{\prime}\left(2 G \hat{\alpha}_{y y}+\hat{\alpha}_{y \theta}\right)\left(\hat{\pi}_{H, t}^{i}+\dot{\tilde{s}}_{t}^{i}\right)}{2 \hat{\alpha}_{\theta \theta}+G \hat{\alpha}_{y \theta}+G\left(2 G \hat{\alpha}_{y y}+\hat{\alpha}_{y \theta}\right)} .
$$

Finally, using (203) and our solution for $\mu_{\pi, t}^{i}$ above, we find

$$
\dot{\mu}_{y, t}^{i}=\rho \mu_{y, t}^{i}+\lambda \frac{\hat{\kappa}_{y} \hat{\Gamma}_{\theta}-\hat{\kappa}_{\theta} \hat{\Gamma}_{y}}{\hat{\kappa}_{\theta}+G \hat{\kappa}_{y}}-\hat{\theta}_{t}^{i} \frac{2 \hat{\kappa}_{y} \hat{\alpha}_{\theta \theta}-\hat{\kappa}_{\theta} \hat{\alpha}_{y \theta}}{\hat{\kappa}_{\theta}+G \hat{\kappa}_{y}}-\hat{\bar{y}}_{t}^{i} \frac{\hat{\alpha}_{y \theta} \hat{\kappa}_{y}-2 \hat{\kappa}_{\theta} \hat{\alpha}_{y y}}{\hat{\kappa}_{\theta}+G \hat{\kappa}_{y}} .
$$

Therefore, we can write the reduced system of optimality conditions just like in the case of optimal transfers as the dynamical system

$$
\begin{aligned}
\dot{\mu}_{y, t}^{i} & =\rho \mu_{y, t}^{i}+\hat{K}_{\theta} \hat{\bar{\theta}}_{t}^{i}+\hat{K}_{y} \hat{\bar{y}}_{t}^{i}+\hat{K}_{\lambda} \lambda \\
\dot{\hat{y}}_{t}^{i} & =\hat{J}_{\mu_{y}} \mu_{y, t}^{i}+\hat{J}_{\pi} \hat{\bar{\pi}}_{H, t}^{i}+\hat{J}_{s} \dot{\tilde{s}}_{t}^{i} \\
\dot{\hat{\theta}}_{t}^{i} & =\hat{H}_{\mu_{y}} \mu_{y, t}^{i}+\hat{H}_{\pi} \hat{\bar{\pi}}_{H, t}^{i}+\hat{H}_{s} \dot{\tilde{s}}_{t}^{i} \\
\dot{\bar{\pi}}_{H, t}^{i} & =\rho \hat{\bar{\pi}}_{H, t}^{i}-\hat{\kappa}_{y} \hat{\bar{y}}_{t}^{i}-\hat{\kappa}_{\theta} \hat{\bar{\theta}}_{t}^{i}
\end{aligned}
$$

subject to the initial conditions $\mu_{y, 0}^{i}=\Delta, \hat{\bar{y}}_{0}^{i}=-\bar{G}^{\prime} \tilde{s}_{0}^{i}+\bar{G} \hat{\theta}_{0}^{i}$.

\section{D.5.2 Solving the Dynamic System}

Viewing the system of optimality conditions as system of linear ODEs, letting $X_{t}^{i}=\left\{\hat{\bar{\pi}}_{H, t}^{i}, \hat{\bar{y}}_{t}^{i}, \hat{\bar{\theta}}_{t}^{i}, \mu_{y, t}^{i}\right\}$, we can write as

$$
\dot{X}_{t}^{i}=A X_{t}^{i}+B_{t}^{i}
$$


where

$$
A=\left(\begin{array}{cccc}
\rho & -\hat{\kappa}_{y} & -\hat{\kappa}_{\theta} & 0 \\
\hat{J}_{\pi} & 0 & 0 & \hat{J}_{\mu_{y}} \\
\hat{H}_{\pi} & 0 & 0 & \hat{H}_{\mu_{y}} \\
0 & \hat{K}_{y} & \hat{K}_{\theta} & \rho
\end{array}\right), \quad B_{t}^{i}=\left(\begin{array}{c}
0 \\
\hat{J}_{t}^{i} \\
\hat{H}_{t}^{i} \\
\hat{K}_{t}^{i}
\end{array}\right), \quad X_{0}^{i}=\left(\begin{array}{c}
\hat{\bar{\pi}}_{H, 0}^{i} \\
\hat{y}_{0}^{i} \\
\hat{\theta}_{0}^{i} \\
\Delta
\end{array}\right),
$$

where $\hat{J}_{t}^{i}=\hat{J}_{s} \dot{\tilde{s}}_{t}^{i}, \hat{H}_{t}^{i}=\hat{H}_{s} \dot{\tilde{s}}_{t}^{i}$ and $\hat{K}_{t}^{i}=\hat{K}_{\theta} \hat{\theta}_{t}^{i}+\hat{K}_{\lambda} \lambda$.

The solution to this dynamical system is given by

$$
X_{t}^{i}=e^{A t}\left[X_{0}^{i}+\int_{0}^{t} e^{-A s} B_{s}^{i} d s\right],
$$

which we can rewrite as

$$
X_{t}^{i}=e^{A t}\left[X_{0}^{i}+\int_{0}^{t} e^{-A s}\left(\hat{\bar{J}}_{s}^{i} E_{2}+\hat{H}_{s}^{i} E_{3}+\hat{\bar{K}}_{s}^{i} E_{4} d s\right],\right.
$$

where $E_{i}$ is the $4 \times 1$ zero vector with a 1 in the $i^{t h}$ position. We rewrite the solution for $X_{t}^{i}$ and get

$$
\begin{aligned}
X_{t}^{i}=e^{A t} & {\left[X_{0}^{i}+\hat{K}_{\lambda} \lambda \int_{0}^{t} e^{-A s} E_{4} d s\right.} \\
& \left.+W_{2} \int_{0}^{t} e^{-A s-\psi s} E_{2} d s+W_{3} \int_{0}^{t} e^{-A s-\psi s} E_{3} d s\right],
\end{aligned}
$$

where now $W_{2}=-\psi a_{0}^{i} \hat{J}_{s}$ and $W_{3}=-\psi a_{0}^{i} \hat{H}_{s}$.

Following the same steps as in the previous section for optimal transfers, we arrive at the following final expression for the solution of the dynamical system of optimality conditions:

$$
\begin{aligned}
X_{t}^{i}= & e^{A t}\left[X_{0}^{i}+\hat{K}_{\lambda} \lambda A^{-1} E_{4}+(A+\psi I)^{-1}\left(W_{2} E_{2}+W_{3} E_{3}\right)\right] \\
& -\hat{K}_{\lambda} \lambda A^{-1} E_{4}-(A+\psi I)^{-1} e^{-\psi t}\left(W_{2} E_{2}+W_{3} E_{3}\right) .
\end{aligned}
$$

\section{D.5.3 Stability}

There is a unique solution to this system of linear ODEs if and only if $A$ has two negative eigenvalues. For stability, we require the term in square brackets above to be in the stable manifold. This gives us four stability conditions, requiring that the term be spanned by the two $4 \times 1$ eigenvectors of $A$ associated with the negative eigenvalues. In particular, we have

$$
\begin{aligned}
0= & \alpha_{1} V_{1}+\alpha_{2} V_{2}+X_{0}^{i}-\kappa_{\theta} \hat{\bar{\theta}}^{i} A^{-1} E_{1}+\left(K_{\theta} \hat{\bar{\theta}}^{i}+K_{\lambda} \lambda\right) A^{-1} E_{4} \\
& +(A+\psi I)^{-1}\left(W_{2} E_{2}+W_{3} E_{3}\right),
\end{aligned}
$$

where, as before, $V_{i}$ is associated with the negative eigenvalue of $A \lambda_{i}$, and $\alpha_{1}, \alpha_{2} \in \mathbb{C}$. To guarantee stability, we solve the vector $Z=\left(\alpha_{1}, \alpha_{2}, \lambda, \Delta, \hat{\bar{\pi}}_{H, 0}^{i}, \hat{\bar{y}}_{0}^{i}, \hat{\bar{\tau}}_{0}^{i, r}\right)^{\prime} \in \mathbb{C}^{7}$ using seven linearly independent 
equations that satisfy said stability criterion as well as the initial conditions of the dynamical system. Equation (218) gives us four of these conditions if we left-multiply both sides by $E_{i}^{\prime}$ for $i \in\{1,2,3,4\}$.

As before, the fifth stability condition will be the initial condition, $\hat{\bar{y}}_{0}^{i}+\bar{G}^{\prime} \tilde{s}_{0}^{i}-\bar{G} \hat{\theta}_{0}^{i}=0$. Using the initial conditions we obtained from the first-order conditions, we can write

$$
0=\left(\hat{\Gamma}_{y} G+\hat{\Gamma}_{\theta}\right) \lambda-\left(2 \hat{\alpha}_{\theta \theta}+G \hat{\alpha}_{y \theta}\right) \hat{\bar{\theta}}_{0}^{i}-\left(2 G \hat{\alpha}_{y y}+\hat{\alpha}_{y \theta}\right) \hat{\bar{y}}_{0}^{i} .
$$

Finally, using the budget constraint, we have

$$
\begin{aligned}
0 & =\int_{0}^{\infty} e^{-\rho t}\left(\hat{\Gamma}_{y} \hat{\bar{y}}_{t}^{i}+\hat{\Gamma}_{\theta} \hat{\bar{\theta}}_{t}^{i}\right) d t \\
& =\int_{0}^{\infty} e^{-\rho t}\left(\hat{\Gamma}_{y} E_{2}+\hat{\Gamma}_{\theta} E_{3}\right)^{\prime} X_{t}^{i} d t .
\end{aligned}
$$

Solving as before yields

$$
\begin{aligned}
0= & \frac{\alpha_{1}}{\lambda_{1}-\rho}\left(\hat{\Gamma}_{y} E_{2}+\hat{\Gamma}_{\theta} E_{3}\right)^{\prime} V_{1}+\frac{\alpha_{2}}{\lambda_{2}-\rho}\left(\hat{\Gamma}_{y} E_{2}+\hat{\Gamma}_{\theta} E_{3}\right)^{\prime} V_{2} \\
& -\frac{\hat{K}_{\lambda} \lambda}{\rho}\left(\hat{\Gamma}_{y} E_{2}+\hat{\Gamma}_{\theta} E_{3}\right)^{\prime} A^{-1} E_{4}-\frac{1}{\rho+\psi}\left(\hat{\Gamma}_{y} E_{2}+\hat{\Gamma}_{\theta} E_{3}\right)^{\prime}(A+\psi I)^{-1}\left(W_{2} E_{2}+W_{3} E_{3}\right) .
\end{aligned}
$$

Putting everything together, we can write $M Z=N$, where

$$
M=\left(\begin{array}{ccccccc}
E_{1}^{\prime} V_{1} & E_{1}^{\prime} V_{2} & \hat{K}_{\lambda} E_{1}^{\prime} A^{-1} E_{4} & 0 & 1 & 0 & 0 \\
E_{2}^{\prime} V_{1} & E_{2}^{\prime} V_{2} & \hat{K}_{\lambda} E_{2}^{\prime} A^{-1} E_{4} & 0 & 0 & 1 & 0 \\
E_{3}^{\prime} V_{1} & E_{3}^{\prime} V_{2} & \hat{K}_{\lambda} E_{3}^{\prime} A^{-1} E_{4} & 0 & 0 & 0 & 1 \\
E_{4}^{\prime} V_{1} & E_{4}^{\prime} V_{2} & \hat{K}_{\lambda} E_{4}^{\prime} A^{-1} E_{4} & 1 & 0 & 0 & 0 \\
0 & 0 & 0 & 0 & 0 & 1 & -\bar{G} \\
0 & 0 & \hat{\Gamma}_{\theta}+G \hat{\Gamma}_{y} & 0 & 0 & -\left(2 \hat{\alpha}_{y y} G+\hat{\alpha}_{y \theta}\right) & -\left(2 \hat{\alpha}_{\theta \theta}+G \hat{\alpha}_{y \theta}\right) \\
\frac{\left(\hat{\Gamma}_{y} E_{2}+\hat{\Gamma}_{\theta} E_{3}\right)^{\prime}}{\lambda_{1}-\rho} V_{1} & \frac{\left(\hat{\Gamma}_{y} E_{2}+\hat{\Gamma}_{\theta} E_{3}\right)^{\prime}}{\lambda_{2}-\rho} V_{2} & -\frac{\hat{K}_{\rho}}{\rho}\left(\hat{\Gamma}_{y} E_{2}+\hat{\Gamma}_{\theta} E_{3}\right)^{\prime} A^{-1} E_{4} & 0 & 0 & 0 & 0
\end{array}\right)
$$

Let $W=(A+\psi I)^{-1}\left(W_{2} E_{2}+W_{3} E_{3}\right)$, then

$$
N=\left(\begin{array}{c}
-E_{1}^{\prime} W \\
-E_{2}^{\prime} W \\
-E_{3}^{\prime} W \\
-E_{4}^{\prime} W \\
-\bar{G}^{\prime} \tilde{s}_{0}^{i} \\
0 \\
\frac{1}{\rho+\psi}\left(\hat{\Gamma}_{y} E_{2}+\hat{\Gamma}_{\theta} E_{3}\right)^{\prime}(A+\psi I)^{-1}\left[W_{2} E_{2}+W_{3} E_{3}\right]
\end{array}\right) .
$$

Given these matrices, we can compute the vector $Z$ numerically by setting 


$$
Z=M^{-1} N
$$

over the parameter subspace on which $M$ is invertible.

\section{D.6 Optimal Government Spending}

Finally, we consider optimal government spending. We set $\hat{\bar{\theta}}_{t}^{i}=\hat{\bar{\theta}}^{i}$ for all $t$ and $N F A_{0}^{i}=0$. This again implies $N \hat{\bar{F}} A_{0}^{i}=0$. Hence, $\tau_{t}^{i, r}=-\left(S_{t}^{i}\right)^{-\alpha} G_{t}^{i}=\tau_{t}^{i, o}$ because the government does not discriminate between agents in the way it sets taxes. Thus, we have $\hat{\bar{\tau}}_{t}^{i, r}=\alpha v \hat{\bar{s}}_{t}^{i}-v \hat{\bar{g}}_{t}^{i}$ or, substituting in for the terms of trade,

$$
\hat{\bar{\tau}}_{t}^{i, r}=\frac{\alpha v}{1-\alpha v v_{\tau}}\left(v_{y} \hat{\bar{y}}_{t}^{i}+\left(v_{g}-\frac{1}{\alpha}\right) \hat{\bar{g}}_{t}^{i}+v_{\theta} \hat{\bar{\theta}}^{i}\right)
$$

NFA. We start by considering country $i$ 's budget constraint and record an important result: For all $\chi \in[0,1]$ and $v \in[0,1]$, we have $\hat{\theta}^{i}=0$ under optimal government spending. Moreover, under the Cole-Obstfeld calibration trade is balanced in every period and HtM agents and optimizers are entirely symmetric.

Under optimal government spending, the budget constraint becomes

$0=\int_{0}^{\infty} e^{-\rho t}\left[\left(\Gamma_{y}+\frac{\alpha v}{1-\alpha v v_{\tau}} v_{y} \Gamma_{\tau}\right) \hat{\bar{y}}_{t}^{i}+\left(\Gamma_{\theta}+\frac{\alpha v}{1-\alpha v v_{\tau}} v_{\theta} \Gamma_{\tau}\right) \hat{\bar{\theta}}^{i}+\left(\Gamma_{g}+\frac{\alpha v}{1-\alpha v v_{\tau}}\left(v_{g}-\frac{1}{\alpha}\right) \Gamma_{\tau}\right) \hat{\hat{g}}_{t}^{i}\right] d t$,

and we have

$$
\begin{aligned}
& \hat{\Gamma}_{y}=\Gamma_{y}+\frac{\alpha v}{1-\alpha v v_{\tau}} v_{y} \Gamma_{\tau}=0 \\
& \hat{\Gamma}_{g}=\Gamma_{g}+\frac{\alpha v}{1-\alpha v v_{\tau}}\left(v_{g}-\frac{1}{\alpha}\right) \Gamma_{\tau}=0 .
\end{aligned}
$$

Therefore, we must have $\hat{\theta}^{i}=0$. Since the term in square brackets is also equivalent to net exports, $\hat{\Gamma}_{y} \hat{\bar{y}}_{t}^{i}+\hat{\Gamma}_{g} \hat{\bar{g}}_{t}^{i}+\hat{\Gamma}_{\theta} \hat{\theta}_{t}^{i}=N \hat{\bar{X}} X_{t}^{i}$, we furthermore find that trade is balanced in every period. This implies that optimizers and HtM agents will have the same consumption profile, since the former do not save or borrow to smooth their consumption over time.

As a result, we can rewrite the expression for the rebate as follows:

$$
\hat{\bar{\tau}}_{t}^{i, r}=\frac{\alpha v}{1-\alpha v v_{\tau}}\left(v_{y} \hat{\hat{y}}_{t}^{i}+\left(v_{g}-\frac{1}{\alpha}\right) \hat{\bar{g}}_{t}^{i}\right) .
$$

And with that, we can now present the simplified dynamical system characterizing the allocation under optimal government spending.

IS equation. The (IS) equation becomes

$$
\dot{\hat{\bar{y}}}_{t}^{i}=-G^{\prime}\left(\hat{\bar{\pi}}_{H, t}^{i}+\dot{\tilde{s}}_{t}^{i}\right)+F \dot{\hat{\bar{g}}}_{t}^{i}
$$


where

$$
F=\frac{\Lambda_{g}+\frac{\alpha v}{1-\alpha v v_{\tau}} \Lambda_{\tau}\left(v_{g}-\frac{1}{\alpha}\right)}{\Lambda_{y}-\frac{\alpha v v_{y}}{1-\alpha v v_{\tau}} \Lambda_{\tau}}
$$

NKPC. The Phillips Curve becomes

$$
\dot{\hat{\bar{\pi}}}_{H, t}^{i}=\rho \hat{\bar{\pi}}_{H, t}^{i}-\hat{\kappa}_{y} \hat{\bar{y}}_{t}^{i}-\hat{\kappa}_{\theta} \hat{\bar{\theta}}^{i}-\hat{\kappa}_{g} \hat{\bar{g}}_{t}^{i}
$$

where

$$
\hat{\kappa}_{g}=\kappa_{g}+\frac{\alpha v}{1-\alpha v v_{\tau}} \kappa_{\tau}\left(v_{g}-\frac{1}{\alpha}\right) .
$$

Initial condition. Finally, we can rewrite the initial condition as

$$
\Lambda_{y} \hat{\bar{y}}_{0}^{i}=-\left(\Lambda_{s}+\alpha v \Lambda_{\tau}\right) \tilde{s}_{0}^{i}+\left(\Lambda_{g}-v \Lambda_{\tau}\right) \hat{g}_{0}^{i}+\Lambda_{\theta} \hat{\bar{\theta}}^{i}
$$

Planning problem. Therefore, the planning problem for country $i$ under optimal government spending becomes

$$
\min _{\left\{\hat{\hat{g}}_{t}^{i}\right\}_{t \geq 0}} \int_{0}^{\infty} e^{-\rho t}\left[\alpha_{\pi}\left(\hat{\bar{\pi}}_{H, t}^{i}\right)^{2}-\left.\hat{\bar{U}}_{t}^{i}\right|_{\hat{\theta}_{t}^{i}=0, \hat{\tau}_{t}^{, i r}=\alpha v \hat{\hat{s}}_{t}^{i}}\right] d t
$$

subject to

$$
\begin{aligned}
\dot{\hat{\bar{y}}}_{t}^{i} & =-G^{\prime}\left(\hat{\bar{\pi}}_{H, t}^{i}+\dot{\tilde{s}}_{t}^{i}\right)+F \dot{\hat{\bar{g}}}_{t}^{i} \\
\dot{\bar{\pi}}_{H, t}^{i} & =\rho \hat{\bar{\pi}}_{H, t}^{i}-\hat{\kappa}_{y} \hat{\bar{y}}_{t}^{i}-\hat{\kappa}_{g} \hat{\bar{g}}_{t}^{i} \\
\Lambda_{y} \hat{\bar{y}}_{0}^{i} & =-\left(\Lambda_{s}+\alpha v \Lambda_{\tau}\right) \tilde{s}_{0}^{i}+\left(\Lambda_{g}-v \Lambda_{\tau}\right) \hat{\bar{g}}_{0}^{i}
\end{aligned}
$$

Control problem. Again, the conversion from planning to optimal control problem requires two steps. First, we now only include the initial condition in the Hamiltonian using Lagrange multiplier $\Delta$, since the isoperimetric budget constraint has dropped out. Second, we substitute out the time derivative $\dot{\hat{g}}_{t}^{i}$ in the (IS) equation and replace it with placeholder $\hat{\bar{v}}_{t}^{i}$. The following Hamiltonian emerges:

$$
\begin{aligned}
H_{t}^{i}\left(x_{t}^{i}, u_{t}^{i}, \mu_{t}^{i}\right)= & \alpha_{\pi}\left(\hat{\bar{\pi}}_{H, t}^{i}\right)^{2}-\left.\hat{\bar{U}}_{t}^{i}\right|_{\hat{\theta}_{t}^{i}=0, \hat{\bar{t}}_{t}^{i, r}=\alpha v \hat{s}_{t}^{i}} \\
& +\Delta\left[\Lambda_{y} \hat{\bar{y}}_{0}^{i}+\left(\Lambda_{s}+\alpha v \Lambda_{\tau}\right) \tilde{s}_{0}^{i}-\left(\Lambda_{g}-v \Lambda_{\tau}\right) \hat{g}_{0}^{i}\right] \\
& +\mu_{y, t}^{i}\left[F \hat{\bar{v}}_{t}^{i}-G^{\prime}\left(\hat{\bar{\pi}}_{H, t}^{i}+\dot{\tilde{s}}_{t}^{i}\right)\right] \\
& +\mu_{g, t}^{i} \hat{\hat{v}}_{t}^{i}
\end{aligned}
$$




$$
+\mu_{\pi, t}^{i}\left[\rho \hat{\bar{\pi}}_{H, t}^{i}-\hat{\kappa}_{y} \hat{\bar{y}}_{t}^{i}-\hat{\kappa}_{g} \hat{\bar{g}}_{t}^{i}\right] .
$$

As before, $x_{t}^{i}$ describes the vector of state variables, $u_{t}^{i}$ the vector of control variables, and $\mu_{t}^{i}$ the vector of costates.

Loss function. Finally, we want to express the simplified loss function under government spending is characterized by

$$
\left.\hat{\bar{U}}_{t}^{i}\right|_{\hat{\bar{\theta}}_{t}^{i}=0, \hat{\tau}_{t}^{i, r}=\alpha v \hat{\bar{s}}_{t}^{i}}=\hat{\alpha}_{y y}\left(\hat{\bar{y}}_{t}^{i}\right)^{2}+\hat{\alpha}_{g g}\left(\hat{\bar{g}}_{t}^{i}\right)^{2}+\hat{\alpha}_{y g} \hat{\bar{y}}_{t}^{i} \hat{\bar{g}}_{t}^{i}
$$

where

$$
\begin{aligned}
& \hat{\alpha}_{g g}=\alpha_{g g}+\left(\frac{\alpha v}{1-\alpha v v_{\tau}}\right)^{2}\left(v_{g}-\frac{1}{\alpha}\right)^{2} \alpha_{\tau \tau}+\frac{\alpha v}{1-\alpha v v_{\tau}}\left(v_{g}-\frac{1}{1-\alpha}\right) \alpha_{g \tau} \\
& \hat{\alpha}_{y g}=2 \alpha_{\tau \tau}\left(\frac{\alpha v}{1-\alpha v v_{\tau}}\right)^{2} v_{y}\left(v_{g}-\frac{1}{\alpha}\right)+\alpha_{y g}+\frac{\alpha v}{1-\alpha v v_{\tau}}\left(v_{g}-\frac{1}{\alpha}\right) \alpha_{y \tau}+\frac{\alpha v}{1-\alpha v v_{\tau}} v_{y} \alpha_{g \tau}
\end{aligned}
$$

and the remaining coefficients are as before.

\section{D.6.1 Optimality Conditions}

The Hamiltonian yields the following first-order conditions.

\section{Optimality:}

$$
F \mu_{y, t}^{i}+\mu_{\tau, t}^{i}=0
$$

\section{Multiplier:}

$$
\begin{gathered}
\dot{\mu}_{\pi, t}^{i}=G^{\prime} \mu_{y, t}^{i}-2 \alpha_{\pi} \hat{\bar{\pi}}_{H, t}^{i} \\
\rho \mu_{y, t}^{i}-\dot{\mu}_{y, t}^{i}=-\hat{\kappa}_{y} \mu_{\pi, t}^{i}-2 \hat{\alpha}_{y y} \hat{\bar{y}}_{t}^{i}-\hat{\alpha}_{y g} \hat{\bar{g}}_{t}^{i} \\
\rho \mu_{g, t}^{i}-\dot{\mu}_{g, t}^{i}=-\hat{\kappa}_{g} \mu_{\pi, t}^{i}-2 \hat{\alpha}_{g g} \hat{\bar{g}}_{t}^{i}-\hat{\alpha}_{y g} \hat{\bar{y}}_{t}^{i}
\end{gathered}
$$

State:

$$
\begin{aligned}
\dot{\hat{\bar{y}}}_{t}^{i} & =-G^{\prime}\left(\hat{\bar{\pi}}_{H, t}^{i}+\dot{\tilde{s}}_{t}^{i}\right)+F \hat{\bar{v}}_{t}^{i} \\
\dot{\hat{\bar{g}}}_{t}^{i} & =\hat{\bar{v}}_{t}^{i} \\
\dot{\overline{\bar{\pi}}}_{H, t}^{i} & =\rho \hat{\bar{\pi}}_{H, t}^{i}-\hat{\kappa}_{y} \hat{\bar{y}}_{t}^{i}-\hat{\kappa}_{g} \hat{\bar{g}}_{t}^{i}
\end{aligned}
$$

\section{Initial Conditions:}

$$
\begin{aligned}
& \mu_{\pi, 0}^{i}=0 \\
& \mu_{y, 0}^{i}=\Delta \Lambda_{y}
\end{aligned}
$$




$$
\mu_{g, 0}^{i}=-\Delta\left(\Lambda_{g}-v \Lambda_{\tau}\right)
$$

We will now simplify the above dynamical system by solving out for $\mu_{\pi, t}^{i}$ and $\mu_{\theta, t}^{i}$. Using equations (228), (230) and (231), we can write

$$
\left(\hat{\kappa}_{g}+F \hat{\kappa}_{y}\right) \mu_{\pi, t}^{i}=-\left(2 \hat{\alpha}_{g g}+F \hat{\alpha}_{y g}\right) \hat{\bar{g}}_{t}^{i}-\left(2 F \hat{\alpha}_{y y}+\alpha_{y g}\right) \hat{\bar{y}}_{t}^{i}
$$

Taking the derivative with respect to time and using (229), we have

$$
\left(\hat{\kappa}_{g}+F \hat{\kappa}_{y}\right)\left(G^{\prime} \mu_{y, t}^{i}-2 \alpha_{\pi} \hat{\bar{\pi}}_{H, t}^{i}\right)+\left(2 \hat{\alpha}_{g g}+F \hat{\alpha}_{y g}\right) \dot{\hat{g}}_{t}^{i}+\left(2 F \hat{\alpha}_{y y}+\hat{\alpha}_{y g}\right) \dot{\hat{y}}_{t}^{i}=0,
$$

from which we can solve for optimal capital controls using the (IS) equation:

$$
\dot{\hat{\bar{g}}}_{t}^{i}=-\frac{\left(\hat{\kappa}_{g}+F \hat{\kappa}_{y}\right)\left(G^{\prime} \mu_{y, t}^{i}-2 \alpha_{\pi} \hat{\pi}_{H, t}^{i}\right)-G^{\prime}\left(2 F \hat{\alpha}_{y y}+\hat{\alpha}_{y g}\right)\left(\hat{\pi}_{H, t}^{i}+\dot{\tilde{s}}_{t}^{i}\right)}{2 \hat{\alpha}_{g g}+F \hat{\alpha}_{y g}+F\left(2 F \hat{\alpha}_{y y}+\hat{\alpha}_{y g}\right)}
$$

Using the (IS) equation again, we have

$$
\dot{\hat{y}}_{t}^{i}=-G^{\prime}\left(\hat{\bar{\pi}}_{H, t}+\dot{\tilde{s}}_{t}^{i}\right)-F \frac{\left(\hat{\kappa}_{g}+F \hat{\kappa}_{y}\right)\left(G^{\prime} \mu_{y, t}^{i}-2 \alpha_{\pi} \hat{\bar{\pi}}_{H, t}^{i}\right)-G^{\prime}\left(2 F \hat{\alpha}_{y y}+\hat{\alpha}_{y g}\right)\left(\hat{\bar{\pi}}_{H, t}^{i}+\dot{\tilde{s}}_{t}^{i}\right)}{2 \hat{\alpha}_{g g}+F \hat{\alpha}_{y g}+F\left(2 F \hat{\alpha}_{y y}+\hat{\alpha}_{y g}\right)} .
$$

Finally, using (230) and our solution for $\mu_{\pi, t}^{i}$ above, we find

$$
\dot{\mu}_{y, t}^{i}=\rho \mu_{y, t}^{i}-\hat{\bar{g}}_{t}^{i} \frac{2 \hat{\kappa}_{y} \hat{\alpha}_{g g}-\hat{\kappa}_{g} \hat{\alpha}_{y g}}{\hat{\kappa}_{g}+F \hat{\kappa}_{y}}-\hat{\bar{y}}_{t}^{i} \frac{\hat{\alpha}_{y g} \hat{\kappa}_{y}-2 \hat{\kappa}_{g} \hat{\alpha}_{y y}}{\hat{\kappa}_{g}+F \hat{\kappa}_{y}} .
$$

Therefore, we can write the reduced system of optimality conditions as

$$
\begin{aligned}
\dot{\mu}_{y, t}^{i} & =\rho \mu_{y, t}^{i}+\tilde{K}_{y} \hat{\bar{y}}_{t}^{i}+\tilde{K}_{g} \hat{\bar{g}}_{t}^{i} \\
\dot{\hat{y}}_{t}^{i} & =\tilde{J}_{\mu_{y}} \mu_{y, t}^{i}+\tilde{J}_{\pi} \hat{\bar{\pi}}_{H, t}^{i}+\tilde{J}_{s} \dot{\tilde{s}}_{t}^{i} \\
\dot{\hat{\theta}}_{t}^{i} & =\tilde{H}_{\mu_{y}} \mu_{y, t}^{i}+\tilde{H}_{\pi} \hat{\bar{\pi}}_{H, t}^{i}+\tilde{H}_{s} \dot{\tilde{s}}_{t}^{i} \\
\dot{\hat{\pi}}_{H, t}^{i} & =\rho \hat{\bar{\pi}}_{H, t}^{i}-\tilde{\kappa}_{y} \hat{\bar{y}}_{t}^{i}-\tilde{\kappa}_{g} \hat{\bar{g}}_{t}^{i}
\end{aligned}
$$

subject to the initial conditions $\mu_{y, 0}^{i}=\Delta \Lambda_{y}$ and $\Lambda_{y} \hat{\bar{y}}_{0}^{i}=-\left(\Lambda_{s}+\alpha v \Lambda_{\tau}\right) \tilde{s}_{0}^{i}+\left(\Lambda_{g}-v \Lambda_{\tau}\right) \hat{\bar{g}}_{0}^{i}$.

\section{D.6.2 Solving the Dynamic System}

Let $X_{t}^{i}=\left\{\hat{\bar{\pi}}_{H, t}^{i}, \hat{\bar{y}}_{t}^{i}, \hat{\bar{g}}_{t}^{i}, \mu_{y, t}^{i}\right\}$. Then we have

$$
\dot{X}_{t}^{i}=A X_{t}^{i}+B_{t}^{i}
$$


where

$$
A=\left(\begin{array}{cccc}
\rho & -\hat{\kappa}_{y} & -\hat{\kappa}_{\theta} & 0 \\
\tilde{J}_{\pi} & 0 & 0 & \tilde{J}_{\mu_{y}} \\
\tilde{H}_{\pi} & 0 & 0 & \tilde{H}_{\mu_{y}} \\
0 & \tilde{K}_{y} & \tilde{K}_{\theta} & \rho
\end{array}\right), \quad B_{t}^{i}=\left(\begin{array}{c}
0 \\
\tilde{J}_{t}^{i} \\
\tilde{H}_{t}^{i} \\
\tilde{K}_{t}^{i}
\end{array}\right), \quad X_{0}^{i}=\left(\begin{array}{c}
\hat{\bar{\pi}}_{H, 0}^{i} \\
\hat{\bar{y}}_{0}^{i} \\
\hat{\bar{\theta}}_{0}^{i} \\
\Delta \Lambda_{y}
\end{array}\right),
$$

where $\tilde{\widetilde{J}}_{t}^{i}=\tilde{J}_{s} \dot{\tilde{s}}_{t}^{i}, \tilde{H}_{t}^{i}=\tilde{H}_{s} \dot{\tilde{S}}_{t}^{i}$, and $\tilde{\bar{K}}_{t}^{i}=\tilde{K}_{\lambda} \lambda$.

Therefore, following the same steps as in the previous two sections the solution of the dynamical system of optimality conditions can be written as

$$
\begin{aligned}
X_{t}^{i}= & e^{A t}\left[X_{0}^{i}+(A+\psi I)^{-1}\left(W_{2} E_{2}+W_{3} E_{3}+W_{4} E_{4}\right)\right] \\
& -(A+\psi I)^{-1} e^{-\psi t}\left(W_{2} E_{2}+W_{3} E_{3}\right),
\end{aligned}
$$

where $W_{2}=-\psi a_{0}^{i} \tilde{J}_{s}$ and $W_{3}=-\psi a_{0}^{i} \tilde{H}_{s}$.

\section{D.6.3 Stability}

As we argued in previous sections, there is a unique solution to this system of linear ODEs if and only if $A$ has two negative eigenvalues. For stability, we require the term in square brackets above to be in the stable manifold. In other words, we require the term in square brackets to be spanned by the two eigenvectors, $V_{1}$ and $V_{2}$, of $A$ that are associated with the two negative eigenvalues, which we will call $\lambda_{1}$ and $\lambda_{2}$. This gives us a system of four linearly independent equations

$$
0=\alpha_{1} V_{1}+\alpha_{2} V_{2}+X_{0}^{i}+(A+\psi I)^{-1}\left(W_{2} E_{2}+W_{3} E_{3}+W_{4} E_{4}\right),
$$

where $\alpha_{1}, \alpha_{2} \in \mathbb{C}$.

Unlike in the previous two sections, we now only have to solve for six variables which we summarize in the vector $Z=\left(\alpha_{1}, \alpha_{2}, \Delta, \hat{\bar{\pi}}_{H, 0}^{i}, \hat{y}_{0}^{i}, \hat{\bar{\tau}}_{0}^{i, r}\right)^{\prime} \in \mathbb{C}^{6}$. Equation (218) gives us four of these conditions if we left-multiply both sides by $E_{i}^{\prime}$ for $i \in\{1,2,3,4\}$.

The fifth stability condition will be the initial condition, $\Lambda_{y} \hat{\bar{y}}_{0}^{i}=-\left(\Lambda_{s}+\alpha v \Lambda_{\tau}\right) \tilde{s}_{0}^{i}+\left(\Lambda_{g}-v \Lambda_{\tau}\right) \hat{\bar{g}}_{0}^{i}$. The sixth and final stability condition we obtain from one of the initial conditions of the dynamical system of optimality conditions,

$$
0=-\left(2 \hat{\alpha}_{g g}+F \hat{\alpha}_{y g}\right) \hat{\bar{g}}_{0}^{i}-\left(2 F \hat{\alpha}_{y y}+\alpha_{y g}\right) \hat{\bar{y}}_{0}^{i}
$$


In matrix form, this system of linear equations can be written $M Z=N$, where

$$
M=\left(\begin{array}{cccccc}
E_{1}^{\prime} V_{1} & E_{1}^{\prime} V_{2} & 0 & 1 & 0 & 0 \\
E_{2}^{\prime} V_{1} & E_{2}^{\prime} V_{2} & 0 & 0 & 1 & 0 \\
E_{3}^{\prime} V_{1} & E_{3}^{\prime} V_{2} & 0 & 0 & 0 & 1 \\
E_{4}^{\prime} V_{1} & E_{4}^{\prime} V_{2} & \Lambda_{y} & 0 & 0 & 0 \\
0 & 0 & 0 & 0 & \Lambda_{y} & -\left(\Lambda_{g}-v \Lambda_{\tau}\right) \\
0 & 0 & 0 & 0 & -\left(2 \hat{\alpha}_{y y} F+\hat{\alpha}_{y g}\right) & -\left(2 \hat{\alpha}_{g g}+F \hat{\alpha}_{y g}\right)
\end{array}\right)
$$

and

$$
N=\left(\begin{array}{c}
-E_{1}^{\prime}(A+\psi I)^{-1}\left(W_{2} E_{2}+W_{3} E_{3}\right) \\
-E_{2}^{\prime}(A+\psi I)^{-1}\left(W_{2} E_{2}+W_{3} E_{3}\right) \\
-E_{3}^{\prime}(A+\psi I)^{-1}\left(W_{2} E_{2}+W_{3} E_{3}\right) \\
-E_{4}^{\prime}(A+\psi I)^{-1}\left(W_{2} E_{2}+W_{3} E_{3}\right) \\
-\left(\Lambda_{s}+\alpha v \Lambda_{\tau}\right) \tilde{s}_{0}^{i} \\
0
\end{array}\right)
$$

Given these matrices, we can compute the vector $Z$ numerically by setting

$$
Z=M^{-1} N
$$

over the parameter subspace on which $M$ is invertible.

\section{D.7 Optimal Redistribution}

We can easily study redistribution as a distinct, fourth policy instrument in the present framework, even though we have not explicitly discussed it until now.

We have previously imposed that the government does not discriminate between agents when funding its own outlays. To introduce redistribution as a distinct policy instrument, we remove this constraint. In addition, we set $N F A_{0}^{i}=0$, implying that country $i$ does not receive international transfers. Therefore, the only relevant constraint becomes

$$
\chi \hat{\bar{\tau}}_{t}^{i, r}+(1-\chi) \hat{\bar{\tau}}_{t}^{i, o}=\alpha v \hat{\bar{s}}_{t}^{i}
$$

since government spending is not used, $\hat{\bar{g}}_{t}^{i}=0$. Finally, the social planner cannot use capital controls, either, so that $\hat{\theta}_{t}^{i}=\hat{\bar{\theta}}^{i}$ for all $t$.

Note that the only important difference between the characterization of optimal redistribution and optimal transfers is country $i$ 's budget constraint. In particular, we now have $N F A_{0}^{i}=0$ so that

$$
0=\int_{0}^{\infty} e^{-\rho t}\left(\hat{\bar{y}}_{t}^{i}-(1-v) \alpha \hat{\hat{s}}_{t}^{i}-(1-v) \hat{\bar{c}}_{t}^{i}\right) d t
$$


where $\hat{N X_{t}^{i}}=\hat{y}_{t}^{i}-(1-v) \alpha \hat{\bar{s}}_{t}^{i}-(1-v) \hat{\bar{c}}_{t}^{i}$. We can rewrite the budget constraint using earlier results so that

$$
0=\int_{0}^{\infty} e^{-\rho t}\left[\left(1-\alpha(1-v) v_{y}-(1-v) \zeta_{y}\right) \hat{\bar{y}}_{t}^{i}-(1-v)\left(\alpha v_{\theta}+\zeta_{\theta}\right) \hat{\bar{\theta}}^{i}-(1-v)\left(\alpha v_{\tau}+\zeta_{\tau}\right) \hat{\bar{\tau}}_{t}^{i, r}\right] d t,
$$

or, more conveniently,

$$
0=\int_{0}^{\infty} e^{-\rho t}\left[\Gamma_{y}^{*} \hat{\bar{y}}_{t}^{i}+\Gamma_{\theta}^{*} \hat{\theta}^{i}+\Gamma_{\tau}^{*} \hat{\bar{\tau}}_{t}^{i, r}\right] d t
$$

\section{D.7.1 Optimal Control Problem}

Exploiting the similarity between transfers and redistribution, we can simply adopt the same loss function, IS equation, Phillips Curve and initial condition. The planning problem can therefore be written as

$$
\min _{\hat{\theta}^{i},\left\{\hat{\hat{t}}_{t}^{i, r}\right\}_{t \geq 0}} \int_{0}^{\infty} e^{-\rho t}\left[\alpha_{\pi}\left(\pi_{H, t}^{i}\right)^{2}-\left.\hat{U}_{t}^{i}\right|_{\hat{\theta}_{t}^{i}=\hat{\theta}^{i}, \hat{g}_{t}^{i}=0}\right] d t,
$$

subject to

$$
\begin{aligned}
\dot{\hat{\bar{y}}}_{t}^{i} & =-D^{\prime}\left(\hat{\bar{\pi}}_{H, t}^{i}+\dot{\tilde{s}}_{t}^{i}\right)+D \hat{\bar{v}}_{t}^{i} \\
\dot{\bar{\tau}}_{t}^{i, r} & =\hat{\bar{v}}_{t}^{i} \\
\dot{\hat{\bar{\tau}}}_{H, t}^{i} & =\rho \hat{\bar{\pi}}_{H, t}^{i}-\kappa_{y} \hat{\bar{y}}_{t}^{i}-\kappa_{\theta} \hat{\bar{\theta}}^{i}-\kappa_{\tau} \hat{\bar{\tau}}_{t}^{i, r}
\end{aligned}
$$

where $D^{\prime}=\frac{\Lambda_{s}}{\Lambda_{y}}$ and $D=\frac{\Lambda_{\tau}}{\Lambda_{y}}$, and also subject to the intial condition $\Lambda_{y} \hat{\bar{y}}_{0}^{i}=-\Lambda_{s} \tilde{s}_{0}^{i}+\Lambda_{\theta} \hat{\bar{\theta}}^{i}+\Lambda_{\tau} \hat{\bar{\tau}}_{0}^{i, r}$. Instead of the budget constraint under transfers, however, we now use the budget constraint presented in (249). As before, the associated optimal control problem can be characterized via the Hamiltonian

$$
\begin{aligned}
H\left(x_{t}^{i}, u_{t}^{i}, \mu_{t}^{i}\right)= & \alpha_{\pi}\left(\pi_{H, t}^{i}\right)^{2}-\left.\hat{\bar{U}}_{t}^{i}\right|_{\hat{\theta}_{t}^{i}=\hat{\theta}^{i}, \hat{\bar{g}}_{t}^{i}=0} \\
& +\lambda\left[\Gamma_{y}^{*} \hat{\bar{y}}_{t}^{i}+\Gamma_{\tau}^{*} \hat{\bar{\tau}}_{t}^{i, r}+\Gamma_{\theta}^{*} \hat{\theta}^{i}\right] \\
& +\Delta\left[\Lambda_{y} \hat{\bar{y}}_{0}^{i}+\Lambda_{s} \tilde{s}_{0}^{i}-\Lambda_{\theta} \hat{\bar{\theta}}^{i}-\Lambda_{\tau} \hat{\bar{\tau}}_{0}^{i, r}\right] \\
& +\mu_{y, t}^{i}\left[D \hat{\bar{v}}_{t}^{i}-D^{\prime}\left(\hat{\bar{\pi}}_{H, t}^{i}+\dot{\hat{s}}_{t}^{i}\right)\right] \\
& +\mu_{\tau, t}^{i} \hat{\bar{v}}_{t}^{i} \\
& +\mu_{\pi, t}^{i}\left[\rho \hat{\bar{\tau}}_{H, t}^{i}-\kappa_{y} \hat{\bar{y}}_{t}^{i}-\kappa_{\theta} \hat{\bar{\theta}}^{i}-\kappa_{\tau} \hat{\bar{\tau}}_{t}^{i, r}\right],
\end{aligned}
$$

where $x_{t}^{i}, u_{t}^{i}$, and $\mu_{t}^{i}$ denote the vectors of state variables, control variables, and costates, respectively.

Reducing the system of the first-order conditions associated with this control problem as before, 
we can summarize the optimal allocation using the dynamical system

$$
\dot{X}_{t}^{i}=A X_{t}^{i}+B_{t}^{i}
$$

where $X_{t}^{i}=\left\{\hat{\bar{\pi}}_{H, t}^{i}, \hat{\bar{y}}_{t}^{i}, \hat{\bar{\tau}}_{t}^{i, r}, \mu_{y, t}^{i}\right\}$ and

$$
A=\left(\begin{array}{cccc}
\rho & -\kappa_{y} & -\kappa_{\tau} & 0 \\
J_{\pi} & 0 & 0 & J_{\mu_{y}} \\
H_{\pi} & 0 & 0 & H_{\mu_{y}} \\
0 & K_{y} & K_{\tau} & \rho
\end{array}\right), \quad B_{t}^{i}=\left(\begin{array}{c}
-\kappa_{\theta} \hat{\bar{\theta}}^{i} \\
\bar{J}_{t}^{i} \\
\bar{H}_{t}^{i} \\
\bar{K}_{t}^{i}
\end{array}\right), \quad X_{0}^{i}=\left(\begin{array}{c}
\hat{\bar{\pi}}_{H, 0}^{i} \\
\hat{\bar{y}}_{0}^{i} \\
\hat{\bar{\tau}}_{0}^{i, r} \\
\Delta \Lambda_{y}
\end{array}\right),
$$

and where $\bar{J}_{t}^{i}=J_{s} \dot{\tilde{s}}_{t}^{i}, \bar{H}_{t}^{i}=H_{s} \dot{\tilde{s}}_{t}^{i}$, and $\bar{K}_{t}^{i}=K_{\theta} \hat{\theta}^{i}+K_{\lambda} \lambda$.

The solution to this dynamical system is, as before, given by

$$
\begin{aligned}
X_{t}^{i}= & e^{A t}\left[X_{0}^{i}-\kappa_{\theta} \hat{\bar{\theta}}^{i} A^{-1} E_{1}+\left(K_{\theta} \hat{\theta}^{i}+K_{\lambda} \lambda\right) A^{-1} E_{4}+(A+\psi I)^{-1}\left(W_{2} E_{2}+W_{3} E_{3}\right)\right] \\
& +\kappa_{\theta} \hat{\theta}^{i} A^{-1} E_{1}-\left(K_{\theta} \hat{\theta}^{i}+K_{\lambda} \lambda\right) A^{-1} E_{4}-(A+\psi I)^{-1} e^{-\psi t}\left(W_{2} E_{2}+W_{3} E_{3}\right)
\end{aligned}
$$

\section{D.7.2 Stability}

To guarantee the stability of this system, we want to solve for the vector $Z=\left(\alpha_{1}, \alpha_{2}, \lambda, \Delta, \hat{\bar{\pi}}_{H, 0^{\prime}}^{i} \hat{\bar{y}}_{0}^{i}, \hat{\bar{\tau}}_{0}^{i, r}\right)^{\prime} \in$ $\mathbb{C}^{7}$ using a set of stability conditions. We use the same first six stability conditions as for optimal transfers. The seventh stability condition, however, is now given by

$$
\begin{aligned}
0= & \frac{\Gamma_{\theta}^{*}}{\rho} \hat{\theta}^{i}+\frac{\alpha_{1}}{\lambda_{1}-\rho}\left(\Gamma_{y}^{*} E_{2}+\Gamma_{\tau}^{*} E_{3}\right)^{\prime} V_{1}+\frac{\alpha_{2}}{\lambda_{2}-\rho}\left(\Gamma_{y}^{*} E_{2}+\Gamma_{\tau}^{*} E_{3}\right)^{\prime} V_{2} \\
& +\frac{\kappa_{\theta} \hat{\theta}^{i}}{\rho}\left(\Gamma_{y}^{*} E_{2}+\Gamma_{\tau}^{*} E_{3}\right)^{\prime} A^{-1} E_{1}-\frac{K_{\theta} \hat{\theta}^{i}+K_{\lambda} \lambda}{\rho}\left(\Gamma_{y}^{*} E_{2}+\Gamma_{\tau}^{*} E_{3}\right)^{\prime} A^{-1} E_{4} \\
& -\frac{1}{\rho+\psi}\left(\Gamma_{y}^{*} E_{2}+\Gamma_{\tau}^{*} E_{3}\right)^{\prime}(A+\psi I)^{-1}\left[W_{2} E_{2}+W_{3} E_{3}\right] .
\end{aligned}
$$

Putting everything together, we can write $M Z=N$, where

$$
M=\left(\begin{array}{ccccccc}
E_{1}^{\prime} V_{1} & E_{1}^{\prime} V_{2} & K_{\lambda} E_{1}^{\prime} A^{-1} E_{4} & 0 & 1 & 0 & 0 \\
E_{2}^{\prime} V_{1} & E_{2}^{\prime} V_{2} & K_{\lambda} E_{2}^{\prime} A^{-1} E_{4} & 0 & 0 & 1 & 0 \\
E_{3}^{\prime} V_{1} & E_{3}^{\prime} V_{2} & K_{\lambda} E_{3}^{\prime} A^{-1} E_{4} & 0 & 0 & 0 & 1 \\
E_{4}^{\prime} V_{1} & E_{4}^{\prime} V_{2} & K_{\lambda} E_{4}^{\prime} A^{-1} E_{4} & \Lambda_{y} & 0 & 0 & 0 \\
0 & 0 & 0 & 0 & 0 & \Lambda_{y} & -\Lambda_{\tau} \\
0 & 0 & \Gamma_{\tau}^{*}+D \Gamma_{y}^{*} & 0 & 0 & -\left(2 \alpha_{y y} D+\alpha_{y \tau}\right) & -\left(2 \alpha_{\tau \tau}+D \alpha_{y \tau}\right) \\
\frac{\left(\Gamma_{y}^{*} E_{2}+\Gamma_{-}^{*} E_{3}\right)^{\prime}}{\lambda_{1}-\rho} V_{1} & \frac{\left(\Gamma_{y}^{*} E_{2}+\Gamma_{\tau}^{*} E_{3}\right)^{\prime}}{\lambda_{2}-\rho} V_{2} & -\frac{K_{\lambda}}{\rho}\left(\Gamma_{y}^{*} E_{2}+\Gamma_{\tau}^{*} E_{3}\right)^{\prime} A^{-1} E_{4} & 0 & 0 & 0 & 0
\end{array}\right)
$$

and 


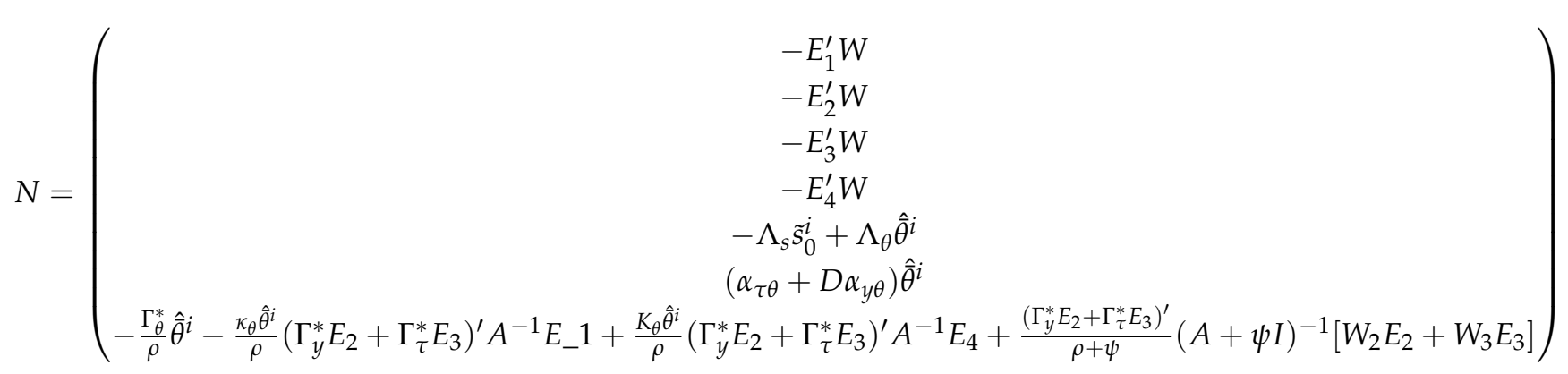

where $W=-\kappa_{\theta} \hat{\theta}^{i} A^{-1} E_{1}+K_{\theta} \hat{\theta}^{i} A^{-1} E_{4}+(A+\psi I)^{-1}\left[W_{2} E_{2}+W_{3} E_{3}\right]$. Given these matrices, we can compute the vector $Z$ numerically by setting

$$
Z=M^{-1} N
$$

over the parameter subspace on which $M$ is invertible.

\section{D.8 Optimal Deficits}

We now study a particular form of deficits in this economy. In particular, we allow the government to accrue debt over time and rebate these funds to households. With the presence of HtM agents, Ricardian equivalence breaks down and such a policy can be effective.

Instead of the per-period government budget constraint, we now impose an isoperimetric constraint of the form

$$
0=\int_{0}^{\infty} e^{-\rho t}\left(\chi \hat{\bar{\tau}}_{t}^{i, r}+(1-\chi) \hat{\bar{\tau}}_{t}^{i, o}+v \hat{\bar{g}}_{t}^{i}-\alpha v \hat{\bar{s}}_{t}^{i}\right) d t .
$$

Implicitly, this constraint states that the government can now accumulate debt from one period to the next. In this section, we assume that $\hat{g}_{t}^{i}=0$ for all $t$ because we want to narrow in on the question of budget deficits, independent from the question of optimal government spending. We furthermore assume that $\hat{\theta}_{t}^{i}=\hat{\theta}^{i}$, since the government cannot use capital controls, and $N F A_{0}^{i}=0$, since we do not want to consider cross-border transfers here.

The second key assumption we make is that the government does not discriminate between agents when rebating its deficit funds, which implies that $\hat{\tau}_{t}^{i, r}=\hat{\tau}_{t}^{i, 0}$. Therefore, the inter-temporal government budget constraint becomes

$$
0=\int_{0}^{\infty} e^{-\rho t}\left(\hat{\bar{\tau}}_{t}^{i, r}-\alpha v \hat{\bar{s}}_{t}^{i}\right) d t
$$

We now consider the problem where the social planner only has access to ex-post transfers, as in body of the paper. In particular, we have $\hat{\theta}_{t}^{i}=\hat{\bar{\theta}}^{i}$ for all $t$ and $\hat{\bar{g}}_{t}^{i}=0$.

IS, NKPC and initial condition. The problem laid out above is almost identical to that of Section 4 with optimal fiscal union transfers. In fact, the only difference in the two planning problems comes from the budget constraint and the now new isoperimetric government budget constraint. In particular, it is easy to verify that we can adopt the same (IS) equation, (NKPC) and initial condition 
as we specified in Section 4. In particular, the (IS) equation is given by

$$
\dot{\hat{y}}_{t}^{i}=-D^{\prime}\left(\hat{\bar{\pi}}_{H, t}^{i}+\dot{\tilde{s}}_{t}^{i}\right)+D \dot{\hat{\bar{\tau}}}_{t}^{i, r} \text {. }
$$

The (NKPC) is given by

$$
\dot{\hat{\pi}}_{H, t}^{i}=\rho \hat{\bar{\pi}}_{H, t}^{i}-\kappa_{y} \hat{\bar{y}}_{t}^{i}-\kappa_{\theta} \hat{\bar{\theta}}^{i}-\kappa_{\tau} \hat{\bar{\tau}}_{t}^{i, r},
$$

and the initial condition is

$$
\Lambda_{y} \hat{\bar{y}}_{0}^{i}=-\Lambda_{s} \tilde{s}_{0}^{i}+\Lambda_{\theta} \hat{\theta}^{i}+\Lambda_{\tau} \hat{\bar{\tau}}_{0}^{i, r} .
$$

NFA. Finally, we rederive country $i$ 's budget constraint. Recall that in the general derivation of the NFA condition in Section 3, we assumed that the government budget constraint held period-byperiod. Under deficit spending, we have

$$
\begin{aligned}
W_{t}^{i} N_{t}^{i, o}-P_{t}^{i} C_{t}^{i, o}+P_{H, t}^{i} Y_{t}^{i}-W_{t}^{i} N_{t}^{i}+P_{t}^{i} \tau_{t}^{i, r} & =W_{t}^{i} N_{t}^{i, o}-P_{t}^{i} C_{t}^{i, o}+P_{H, t}^{i} Y_{t}^{i}-W_{t}^{i} N_{t}^{i}+P_{t}^{i} T_{t}^{i, r}-P_{t}^{i} Z_{H, t}^{i} \\
& =W_{t}^{i} N_{t}^{i, o}-P_{t}^{i} C_{t}^{i, o}+P_{t}^{i} C_{t}^{i, r}-W_{t}^{i} N_{t}^{i, r} \\
& =P_{t}^{i}\left(C_{t}^{i, r}-C_{t}^{i, o}\right)-W_{t}^{i}\left(N_{t}^{i, r}-N_{t}^{i, o}\right) .
\end{aligned}
$$

Therefore, we have

$$
\begin{aligned}
N F A_{0}^{i} & =-\int_{0}^{\infty} e^{-\rho t} \frac{1}{P_{F}^{i}}\left[P_{t}^{i}\left(C_{t}^{i, r}-C_{t}^{i, o}\right)-W_{t}^{i}\left(N_{t}^{i, r}-N_{t}^{i, o}\right)\right] d t \\
& =-\int_{0}^{\infty} e^{-\rho t}\left[\left(S_{t}^{i}\right)^{-(1-\alpha)}\left(C_{t}^{i, r}-C_{t}^{i, o}\right)-\frac{1}{1-v} C_{t}^{i, r}\left(N_{t}^{i, r}\right)^{\phi}\left(S_{t}^{i}\right)^{-(1-\alpha)}\left(N_{t}^{i, r}-N_{t}^{i, o}\right)\right] d t
\end{aligned}
$$

since

$$
\frac{W_{t}^{i}}{P_{F}^{i}}=\frac{W_{t}^{i}}{P_{t}^{i}} \frac{P_{t}^{i}}{P_{F}^{i}}=\frac{1}{1-v} C_{t}^{i, r}\left(N_{t}^{i, r}\right)^{\phi} \frac{P_{t}^{i}}{P_{H, t}^{i}} \frac{P_{H, t}^{i}}{P_{F}^{i}}=\frac{1}{1-v} C_{t}^{i, r}\left(N_{t}^{i, r}\right)^{\phi}\left(S_{t}^{i}\right)^{-(1-\alpha)} .
$$

Linearizing, we find

$$
N F A_{0}^{i}=-\int_{0}^{\infty} e^{-\rho t}\left[(1-v)\left(c_{t}^{i, r}-c_{t}^{i, o}\right)-\left(n_{t}^{i, r}-n_{t}^{i, o}\right)\right] d t
$$

Adopting gap notation and noting that $N \hat{\bar{F}} A_{0}^{i}=0$,

$$
\begin{aligned}
0= & \int_{0}^{\infty} e^{-\rho t}\left[(1-v)\left(\hat{\bar{c}}_{t}^{i, r}-\hat{\bar{c}}_{t}^{i, o}\right)-\left(\hat{\bar{n}}_{t}^{i, r}-\hat{\bar{n}}_{t}^{i, o}\right)\right] d t \\
= & \int_{0}^{\infty} e^{-\rho t}\left[(1-v)\left(\hat{\bar{c}}_{t}^{i, r}-\hat{\bar{\theta}}^{i}-(1-\alpha) \hat{\hat{s}}_{t}^{i}\right)-\left(\hat{\bar{n}}_{t}^{i, r}-\frac{1}{1-\chi} \hat{\bar{n}}_{t}^{i}+\frac{\chi}{1-\chi} \hat{\bar{n}}_{t}^{i, r}\right)\right] d t \\
= & \int_{0}^{\infty} e^{-\rho t}\left[(1-v)\left(\psi_{y} \hat{\bar{y}}_{t}^{i}+\left(\psi_{\theta}-1\right) \hat{\bar{\theta}}^{i}+\psi_{\tau} \hat{\bar{\tau}}_{t}^{i, r}\right)+\frac{1}{1-\chi} \hat{\bar{y}}_{t}^{i}\right. \\
& \left.-(1-\alpha)(1-v)\left(v_{y} \hat{\bar{y}}_{t}^{i}+v_{\theta} \hat{\bar{\theta}}^{i}+v_{\tau} \hat{\bar{\tau}}_{t}^{i, r}\right)-\frac{1}{1-\chi}\left(\eta_{y} \hat{\bar{y}}_{t}^{i}+\eta_{\theta} \hat{\bar{\theta}}^{i}+\eta_{\tau} \hat{\bar{\tau}}_{t}^{i, r}\right)\right] d t .
\end{aligned}
$$


To simplify notation, we write

$$
0=\int_{0}^{\infty} e^{-\rho t}\left[\Gamma_{y}^{* *} \hat{\bar{y}}_{t}^{i}+\Gamma_{\theta}^{* *} \hat{\theta}^{i}+\Gamma_{\tau}^{* *} \hat{\bar{\tau}}_{t}^{i, r}\right] d t .
$$

Partial collinearity of NFA and GBC. Comparing the NFA condition with the isoperimetric government budget constraint, which we can write as

$$
0=\int_{0}^{\infty} e^{-\rho t}\left[\left(1-\alpha v v_{\tau}\right) \hat{\bar{\tau}}_{t}^{i, r}-\alpha v v_{y} \hat{\hat{y}}_{t}^{i}-\alpha v v_{\theta} \hat{\theta}^{i}\right] d t,
$$

we note that we have

$$
\frac{\Gamma_{y}^{* *}}{-\alpha v v_{y}}=\frac{\Gamma_{\tau}^{* *}}{1-\alpha v v_{\tau}} \equiv v .
$$

We can multiply (259) by $1 / v$ and subtract it from (260). This yields

$$
0=\int_{0}^{\infty} e^{-\rho t}\left[-\alpha v v_{\theta} \hat{\bar{\theta}}^{i}-\frac{\Gamma_{\theta}^{* *}}{v} \hat{\bar{\theta}}^{i}\right] d t
$$

which immediately implies that we must have $\hat{\theta}^{i}=0$.

Planning problem. Country $i$ 's planning problem can therefore be written as

$$
\min _{\hat{\theta}^{i},\left\{\hat{\tau}_{t}^{i, r}\right\} t \geq 0} \int_{0}^{\infty} e^{-\rho t}\left[\alpha_{\pi}\left(\hat{\bar{\pi}}_{H, t}^{i}\right)^{2}-\left.\hat{\bar{U}}_{t}^{i}\right|_{\hat{\bar{\theta}}_{t}^{i}=0, \hat{\bar{s}}_{t}^{i}=0}\right] d t,
$$

subject to

$$
\begin{aligned}
\dot{\hat{\bar{y}}}_{t}^{i} & =-D^{\prime}\left(\hat{\bar{\pi}}_{H, t}^{i}+\dot{\tilde{s}}_{t}^{i}\right)+D \dot{\hat{\bar{\tau}}}_{t}^{i, r} \\
\dot{\hat{\pi}}_{H, t}^{i} & =\rho \hat{\bar{\pi}}_{H, t}^{i}-\kappa_{y} \hat{\bar{y}}_{t}^{i}-\kappa_{\tau} \hat{\bar{\tau}}_{t}^{i, r} \\
\Lambda_{y} \hat{\bar{y}}_{0}^{i} & =-\Lambda_{s} \tilde{s}_{0}^{i}+\Lambda_{\tau} \hat{\bar{\tau}}_{0}^{i, r} \\
0 & =\int_{0}^{\infty} e^{-\rho t}\left[\Gamma_{y}^{* *} \hat{\bar{y}}_{t}^{i}+\Gamma_{\tau}^{* *} \hat{\bar{\tau}}_{t}^{i, r}\right] d t
\end{aligned}
$$

where $D^{\prime}=\frac{\Lambda_{s}}{\Lambda_{y}}$ and $D=\frac{\Lambda_{\tau}}{\Lambda_{y}}$, and

$$
\left.\hat{U}_{t}^{i}\right|_{\hat{\theta}_{t}^{i}=0, \hat{\bar{g}}_{t}^{i}=0}=\alpha_{y y}\left(\hat{\bar{y}}_{t}^{i}\right)^{2}+\alpha_{\tau \tau}\left(\hat{\bar{\tau}}_{t}^{i, r}\right)^{2}+\alpha_{y \tau} \hat{\bar{y}}_{t}^{i} \hat{\bar{\tau}}_{t}^{i, r}
$$

Control problem. To transform this planning problem into an optimal control problem that we can work with we replace the time derivative $\dot{\hat{\tau}}_{t}^{i, r}$ in the (IS) equation by a placeholder, $\hat{\bar{v}}_{t}^{i}$, and introduce a new dynamic state equation. The Hamiltonian associated with this control problem can then be 
written as

$$
\begin{aligned}
H\left(x_{t}^{i}, u_{t}^{i}, \mu_{t}^{i}\right)= & \alpha_{\pi}\left(\hat{\bar{\tau}}_{H, t}^{i}\right)^{2}-\left.\hat{\bar{U}}_{t}^{i}\right|_{\hat{\bar{\theta}}_{t}^{i}=0, \hat{\bar{g}}_{t}^{i}=0} \\
& +\Delta\left[\Lambda_{y} \hat{\bar{y}}_{0}^{i}+\Lambda_{s} \tilde{s}_{0}^{i}-\Lambda_{\tau} \hat{\bar{\tau}}_{0}^{i, r}\right] \\
& +\lambda\left[\Gamma_{y}^{* *} \hat{\bar{y}}_{t}^{i}+\Gamma_{\tau}^{* *} \hat{\bar{\tau}}_{t}^{i, r}\right] \\
& +\mu_{y, t}^{i}\left[D \hat{\bar{v}}_{t}^{i}-D^{\prime}\left(\hat{\bar{\pi}}_{H, t}^{i}+\dot{\tilde{s}}_{t}^{i}\right)\right] \\
& +\mu_{\tau, t}^{i} \hat{\bar{v}}_{t}^{i} \\
& +\mu_{\pi, t}^{i}\left[\rho \hat{\bar{\pi}}_{H, t}^{i}-\kappa_{y} \hat{\bar{y}}_{t}^{i}-\kappa_{\tau} \hat{\bar{\tau}}_{t}^{i, r}\right],
\end{aligned}
$$

where $x_{t}^{i}=\left\{\hat{\bar{\pi}}_{H, t}^{i}, \hat{\bar{y}}_{t}^{i}, \hat{\bar{\tau}}_{t}^{i, r}\right\}$ denotes the vector of state variables, $u_{t}^{i}=\left\{\hat{\bar{v}}_{t}^{i}\right\}$ the vector of control variables, and $\mu_{t}^{i}=\left\{\mu_{\pi, t}^{i}, \mu_{y, t}^{i}, \mu_{\tau, t}^{i}\right\}$ the vector of costates.

\section{D.8.1 Optimality Conditions}

The Hamiltonian yields the nine first-order conditions

\section{Optimality:}

$$
D \mu_{y, t}^{i}+\mu_{\tau, t}^{i}=0
$$

\section{Multiplier:}

$$
\begin{aligned}
\dot{\mu}_{\pi, t}^{i} & =D^{\prime} \mu_{y, t}^{i}-2 \alpha_{\pi} \hat{\bar{\pi}}_{H, t}^{i} \\
\rho \mu_{y, t}^{i}-\dot{\mu}_{y, t}^{i} & =\Gamma_{y}^{* *} \lambda-\kappa_{y} \mu_{\pi, t}^{i}-2 \alpha_{y y} \hat{\bar{y}}_{t}^{i}-\alpha_{y \tau} \hat{\bar{\tau}}_{t}^{i, r} \\
\rho \mu_{\tau, t}^{i}-\dot{\mu}_{\tau, t}^{i} & =\Gamma_{\tau}^{* *} \lambda-\kappa_{\tau} \mu_{\pi, t}^{i}-2 \alpha_{\tau \tau} \hat{\bar{\tau}}_{t}^{i, r}-\alpha_{y \tau} \hat{\bar{y}}_{t}^{i}
\end{aligned}
$$

State:

$$
\begin{aligned}
\dot{\hat{\bar{y}}}_{t}^{i} & =-D^{\prime}\left(\hat{\bar{\pi}}_{H, t}^{i}+\dot{\tilde{s}}_{t}^{i}\right)+D \hat{\bar{v}}_{t}^{i} \\
\dot{\hat{\bar{\tau}}}_{t}^{i, r} & =\hat{\bar{v}}_{t}^{i} \\
\dot{\hat{\bar{\tau}}}_{H, t}^{i} & =\rho \hat{\bar{\pi}}_{H, t}^{i}-\kappa_{y} \hat{\bar{y}}_{t}^{i}-\kappa_{\tau} \hat{\bar{\tau}}_{t}^{i, r}
\end{aligned}
$$

\section{Initial conditions:}

$$
\begin{aligned}
& \mu_{\pi, 0}^{i}=0 \\
& \mu_{y, 0}^{i}=\Delta \Lambda_{y}
\end{aligned}
$$




$$
\mu_{\tau, 0}^{i}=-\Delta \Lambda_{\tau}
$$

We can now solve out for $\mu_{\pi, t}^{i}$ and $\mu_{\tau, t}^{i}$ to reduce the dimensionality of this dynamical system. Using equations (264), (266) and (267), we can write

$$
\left(\kappa_{\tau}+D \kappa_{y}\right) \mu_{\pi, t}^{i}=\left(\Gamma_{y}^{* *} D+\Gamma_{\tau}^{* *}\right) \lambda-\left(2 \alpha_{\tau \tau}+D \alpha_{y \tau}\right) \hat{\bar{\tau}}_{t}^{i, r}-\left(2 D \alpha_{y y}+\alpha_{y \tau}\right) \hat{\bar{y}}_{t}^{i} .
$$

Taking the derivative with respect to time and using (265), we have

$$
\left(\kappa_{\tau}+D \kappa_{y}\right)\left(D^{\prime} \mu_{y, t}^{i}-2 \alpha_{\pi} \hat{\bar{\pi}}_{H, t}^{i}\right)+\left(2 \alpha_{\tau \tau}+D \alpha_{y \tau}\right) \dot{\hat{\tau}}_{t}^{i, r}+\left(2 D \alpha_{y y}+\alpha_{y \tau}\right) \dot{\hat{y}}_{t}^{i}=0,
$$

from which we can solve for the rate of change of transfers using the (IS) equation:

$$
\dot{\hat{\tau}}_{t}^{i, r}=-\frac{\left(\kappa_{\tau}+D \kappa_{y}\right)\left(D^{\prime} \mu_{y, t}^{i}-2 \alpha_{\pi} \hat{\bar{\pi}}_{H, t}^{i}\right)-D^{\prime}\left(2 D \alpha_{y y}+\alpha_{y \tau}\right)\left(\hat{\bar{\pi}}_{H, t}^{i}+\dot{\tilde{s}}_{t}^{i}\right)}{2 \alpha_{\tau \tau}+D \alpha_{y \tau}+D\left(2 D \alpha_{y y}+\alpha_{y \tau}\right)}
$$

Using the (IS) equation again, we have

$$
\dot{\hat{y}}_{t}^{i}=-D^{\prime}\left(\hat{\bar{\pi}}_{H, t}^{i}+\dot{\tilde{s}}_{t}^{i}\right)-D \frac{\left(\kappa_{\tau}+D \kappa_{y}\right)\left(D^{\prime} \mu_{y, t}^{i}-2 \alpha_{\pi} \hat{\bar{\pi}}_{H, t}^{i}\right)-D^{\prime}\left(2 D \alpha_{y y}+\alpha_{y \tau}\right)\left(\hat{\bar{\pi}}_{H, t}^{i}+\dot{\tilde{s}}_{t}^{i}\right)}{2 \alpha_{\tau \tau}+D \alpha_{y \tau}+D\left(2 D \alpha_{y y}+\alpha_{y \tau}\right)} .
$$

Finally, using (266) and our solution for $\mu_{\pi, t}^{i}$ above, we find

$$
\dot{\mu}_{y, t}^{i}=\rho \mu_{y, t}^{i}+\lambda \frac{\kappa_{y} \Gamma_{\tau}^{* *}-\kappa_{\tau} \Gamma_{y}^{* *}}{\kappa_{\tau}+D \kappa_{y}}-\hat{\bar{\tau}}_{t}^{i, r} \frac{2 \kappa_{y} \alpha_{\tau \tau}-\kappa_{\tau} \alpha_{y \tau}}{\kappa_{\tau}+D \kappa_{y}}-\hat{\bar{y}}_{t}^{i} \frac{\alpha_{y \tau} \kappa_{y}-2 \kappa_{\tau} \alpha_{y y}}{\kappa_{\tau}+D \kappa_{y}} .
$$

Therefore, we can write the reduced system of optimality conditions as

$$
\begin{aligned}
\dot{\mu}_{y, t}^{i} & =\rho \mu_{y, t}^{i}+K_{\tau} \hat{\bar{\tau}}_{t}^{i, r}+K_{y} \hat{\bar{y}}_{t}^{i}+K_{\lambda} \lambda \\
\dot{\hat{y}}_{t}^{i} & =J_{\mu_{y}} \mu_{y, t}^{i}+J_{\pi} \hat{\pi}_{H, t}^{i}+J_{s} \dot{\tilde{s}}_{t}^{i} \\
\dot{\bar{\tau}}_{t}^{i, r} & =H_{\mu_{y}} \mu_{y, t}^{i}+H_{\pi} \hat{\bar{\pi}}_{H, t}^{i}+H_{s} \dot{\tilde{s}}_{t}^{i} \\
\dot{\bar{\pi}}_{H, t}^{i} & =\rho \hat{\bar{\pi}}_{H, t}^{i}-\kappa_{y} \hat{\bar{y}}_{t}^{i}-\kappa_{\tau} \hat{\bar{\tau}}_{t}^{i, r} .
\end{aligned}
$$

\section{D.8.2 Solving the Dynamical System}

We can thus express the dynamical system of first-order conditions as a system of linear ODEs,

$$
\dot{X}_{t}^{i}=A X_{t}^{i}+B_{t}^{i}
$$


for $X_{t}^{i}=\left\{\hat{\bar{\pi}}_{H, t}^{i}, \hat{\bar{y}}_{t}^{i}, \hat{\bar{\tau}}_{t}^{i, r}, \mu_{y, t}^{i}\right\}$. We have

$$
A=\left(\begin{array}{cccc}
\rho & -\kappa_{y} & -\kappa_{\tau} & 0 \\
J_{\pi} & 0 & 0 & J_{\mu_{y}} \\
H_{\pi} & 0 & 0 & H_{\mu_{y}} \\
0 & K_{y} & K_{\tau} & \rho
\end{array}\right), \quad B_{t}^{i}=\left(\begin{array}{c}
0 \\
\bar{J}_{t}^{i} \\
\bar{H}_{t}^{i} \\
\bar{K}_{t}^{i}
\end{array}\right), \quad X_{0}^{i}=\left(\begin{array}{c}
\hat{\bar{\pi}}_{H, 0}^{i} \\
\hat{\bar{y}}_{0}^{i} \\
\hat{\bar{\tau}}_{0}^{i, r} \\
\Delta \Lambda_{y}
\end{array}\right),
$$

and $\bar{J}_{t}^{i}=J_{s} \dot{\tilde{s}}_{t}^{i}, \bar{H}_{t}^{i}=H_{s} \dot{\tilde{s}}_{t}^{i}$ and $\bar{K}_{t}^{i}=K_{\lambda} \lambda$.

Following the same steps as in Section 4, we can write the solution as

$$
X_{t}^{i}=e^{A t}\left[X_{0}^{i}+K_{\lambda} \lambda \int_{0}^{t} e^{-A s} E_{4} d s+W_{2} \int_{0}^{t} e^{-A s-\psi s} E_{2} d s+W_{3} \int_{0}^{t} e^{-A s-\psi s} E_{3} d s\right],
$$

where $W_{2}=-\psi a_{0}^{i} J_{s}$ and $W_{3}=-\psi a_{0}^{i} H_{s}$. Assuming that the economy is in the parameter subspace where $A$ is nonsingular, we can solve out the integrals to arrive at

$$
\begin{aligned}
X_{t}^{i}= & e^{A t}\left[X_{0}^{i}+K_{\lambda} \lambda A^{-1} E_{4}+(A+\psi I)^{-1}\left(W_{2} E_{2}+W_{3} E_{3}\right)\right] \\
& -K_{\lambda} \lambda A^{-1} E_{4}-(A+\psi I)^{-1} e^{-\psi t}\left(W_{2} E_{2}+W_{3} E_{3}\right) .
\end{aligned}
$$

\section{D.8.3 Stability}

Our main departure from the solution in Section 4 is the following stability analysis. To guarantee uniqueness, we still require

$$
X_{0}^{i}+K_{\lambda} \lambda A^{-1} E_{4}+(A+\psi I)^{-1}\left(W_{2} E_{2}+W_{3} E_{3}\right)
$$

to be in the stable manifold. Let $V_{j}, j \in\{1,2\}$, denote the eigenvector of $A$ associated with the negative eigenvalue $\lambda_{j}$. We can express this stability condition as

$$
0=\alpha_{1} V_{1}+\alpha_{2} V_{2}+X_{0}^{i}+K_{\lambda} \lambda A^{-1} E_{4}+(A+\psi I)^{-1}\left(W_{2} E_{2}+W_{3} E_{3}\right),
$$

for some $\alpha_{j} \in \mathbb{C}, j \in\{1,2\}$.

Under deficit spending, however, we now have an extra parameter that we need to solve for. That is, we now need to solve the vector $Z=\left(\alpha_{1}, \alpha_{2}, \lambda, \Delta, \hat{\bar{\pi}}_{H, 0}^{i}, \hat{\bar{y}}_{0}^{i}, \hat{\bar{\tau}}_{0}^{i, r}\right)^{\prime} \in \mathbb{C}^{7}$ to satisfy the stability requirement and initial conditions. Equation (278) yields four of the desired eight conditions. As before, we left-multiply (278) by $E_{i}^{\prime}$ for each $i \in\{1,2,3,4\}$.

The first initial condition we can use is $\Lambda_{y} \hat{\bar{y}}_{0}^{i}=-\Lambda_{s} \tilde{s}_{0}^{i}+\Lambda_{\tau} \hat{\bar{\tau}}_{0}^{i, r}$. Another one we obtain by writing

$$
\left(\kappa_{\tau}+D \kappa_{y}\right) \mu_{\pi, 0}^{i}=0=\left(\Gamma_{y}^{* *} D+\Gamma_{\tau}^{* *}\right) \lambda-\left(2 \alpha_{\tau \tau}+D \alpha_{y \tau}\right) \hat{\bar{\tau}}_{0}^{i, r}-\left(2 D \alpha_{y y}+\alpha_{y \tau}\right) \hat{y}_{0}^{i} .
$$

The remaining two conditions we obtain from the two constraints associated with $\lambda_{1}$ and $\lambda_{2}$, 
respectively. Note that we can write

$$
\begin{aligned}
X_{t}^{i} & =e^{A t}\left(-\alpha_{1} V_{1}-\alpha_{2} V_{2}\right)-K_{\lambda} \lambda A^{-1} E_{4}-(A+\psi I)^{-1} e^{-\psi t}\left(W_{2} E_{2}+W_{3} E_{3}\right) \\
& =-\alpha_{1} e^{\lambda_{1} t} V_{1}-\alpha_{2} e^{\lambda_{2} t} V_{2}-K_{\lambda} \lambda A^{-1} E_{4}-(A+\psi I)^{-1} e^{-\psi t}\left(W_{2} E_{2}+W_{3} E_{3}\right) .
\end{aligned}
$$

The budget constraint then becomes

$$
\begin{aligned}
0 & =\int_{0}^{\infty} e^{-\rho t}\left[\Gamma_{y}^{* *} \hat{\bar{y}}_{t}^{i}+\Gamma_{\tau}^{* *} \hat{\bar{\tau}}_{t}^{i, r}\right] d t \\
& =\int_{0}^{\infty} e^{-\rho t}\left(\Gamma_{y}^{* *} E_{2}+\Gamma_{\tau}^{* *} E_{3}\right)^{\prime} X_{t}^{i} d t
\end{aligned}
$$

where we can plug in the solution for $X_{t}^{i}$ and solve out the integrals to obtain

$$
\begin{aligned}
0= & \frac{\alpha_{1}}{\lambda_{1}-\rho}\left(\Gamma_{y}^{* *} E_{2}+\Gamma_{\tau}^{* *} E_{3}\right)^{\prime} V_{1}+\frac{\alpha_{2}}{\lambda_{2}-\rho}\left(\Gamma_{y}^{* *} E_{2}+\Gamma_{\tau}^{* *} E_{3}\right)^{\prime} V_{2} \\
& -\frac{K_{\lambda} \lambda}{\rho}\left(\Gamma_{y}^{* *} E_{2}+\Gamma_{\tau}^{* *} E_{3}\right)^{\prime} A^{-1} E_{4} \\
& -\frac{1}{\rho+\psi}\left(\Gamma_{y}^{* *} E_{2}+\Gamma_{\tau}^{* *} E_{3}\right)^{\prime}(A+\psi I)^{-1}\left[W_{2} E_{2}+W_{3} E_{3}\right] .
\end{aligned}
$$

Putting everything together, we can write $M Z=N$. Letting $\tilde{E}=\left(\Gamma_{y}^{* *} E_{2}+\Gamma_{\tau}^{* *} E_{3}\right)$ for ease of notation, we have

$$
M=\left(\begin{array}{ccccccc}
E_{1}^{\prime} V_{1} & E_{1}^{\prime} V_{2} & K_{\lambda} E_{1}^{\prime} A^{-1} E_{4} & 0 & 1 & 0 & 0 \\
E_{2}^{\prime} V_{1} & E_{2}^{\prime} V_{2} & K_{\lambda} E_{2}^{\prime} A^{-1} E_{4} & 0 & 0 & 1 & 0 \\
E_{3}^{\prime} V_{1} & E_{3}^{\prime} V_{2} & K_{\lambda} E_{3}^{\prime} A^{-1} E_{4} & 0 & 0 & 0 & 1 \\
E_{4}^{\prime} V_{1} & E_{4}^{\prime} V_{2} & K_{\lambda} E_{4}^{\prime} A^{-1} E_{4} & \Lambda_{y} & 0 & 0 & 0 \\
0 & 0 & 0 & 0 & 0 & \Lambda_{y} & -\Lambda_{\tau} \\
0 & 0 & \Gamma_{\tau}^{* *}+D \Gamma_{y}^{* *} & 0 & 0 & -\left(2 \alpha_{y y} D+\alpha_{y \tau}\right) & -\left(2 \alpha_{\tau \tau}+D \alpha_{y \tau}\right) \\
\frac{\tilde{E}^{\prime}}{\lambda_{1}-\rho} V_{1} & \frac{\tilde{E}^{\prime}}{\lambda_{2}-\rho} V_{2} & -\frac{K_{\lambda}}{\rho} \tilde{E}^{\prime} A^{-1} E_{4} & 0 & 0 & 0 & 0
\end{array}\right)
$$

and

$$
N=\left(\begin{array}{c}
-E_{1}^{\prime} W \\
-E_{2}^{\prime} W \\
-E_{3}^{\prime} W \\
-E_{4}^{\prime} W \\
-\Lambda_{s} \tilde{S}_{0}^{i} \\
0 \\
\frac{\tilde{E}^{\prime}}{\rho+\psi}(A+\psi I)^{-1}\left[W_{2} E_{2}+W_{3} E_{3}\right]
\end{array}\right)
$$


where $W=(A+\psi I)^{-1}\left[W_{2} E_{2}+W_{3} E_{3}\right]$. Given these matrices, we can compute the vector $Z$ numerically by setting

$$
\mathrm{Z}=M^{-1} N
$$

over the parameter subspace on which $M$ is invertible.

\section{D.9 Jointly Optimal Fiscal Policy}

Finally, we consider optimal fiscal policy, allowing for both government spending and redistribution. The allocation derived under jointly optimal government and deficit spending would be identical.

In the absence of international transfers, we have $N F A_{0}^{i}=N \hat{\bar{F}} A_{0}^{i}=0$. We do not allow for capital controls, $\hat{\theta}_{t}^{i}=\hat{\theta}^{i}$, and impose the per-period government budget constraint, $\chi \hat{\bar{\tau}}_{t}^{i, r}+(1-\chi) \hat{\bar{\tau}}_{t}^{i, o}+$ $v \hat{\bar{g}}_{t}^{i}-\alpha v \hat{\bar{s}}_{t}^{i}=0$.

Since the government's budget is balanced in every period, the original external budget constraint for country $i$ holds,

$$
0=\int_{0}^{\infty} e^{\rho t}\left[\hat{\bar{y}}_{t}^{i}-\alpha(1-v) \hat{\bar{s}}_{t}^{i}-(1-v) \hat{\bar{c}}_{t}^{i}-v \hat{\bar{g}}_{t}^{i}\right] d t \equiv \int_{0}^{\infty} e^{-\rho t} \hat{N X_{t}^{i}} d t
$$

where as before $\hat{\bar{s}}_{t}^{i}=v_{y} \hat{\bar{y}}_{t}^{i}+v_{\tau} \hat{\bar{\tau}}_{t}^{i, r}+v_{\theta} \hat{\bar{\theta}}^{i}+v_{g} \hat{\bar{g}}_{t}^{i}$ and $\hat{\bar{c}}_{t}^{i}=\zeta_{y} \hat{\bar{y}}_{t}^{i}+\zeta_{g} \hat{\bar{g}}_{t}^{i}+\zeta_{\tau} \hat{\bar{\tau}}_{t}^{i, r}+\zeta_{\theta} \hat{\bar{\theta}}^{i}$. We can simplify to write the NFA condition as

$$
0=\int_{0}^{\infty} e^{-\rho t}\left[\Gamma_{y}^{*} \hat{\bar{y}}_{t}^{i}+\Gamma_{\theta}^{*} \hat{\bar{\theta}}^{i}+\Gamma_{\tau}^{*} \hat{\bar{t}}_{t}^{i, r}+\Gamma_{g}^{*} \hat{\bar{g}}_{t}^{i}\right] d t
$$

where

$$
\begin{aligned}
\Gamma_{y}^{*} & =1-\alpha(1-v) v_{y}-(1-v) \zeta_{y} \\
\Gamma_{\theta}^{*} & =-\alpha(1-v) v_{\tau}-(1-v) \zeta_{\tau} \\
\Gamma_{\tau}^{*} & =-\alpha(1-v) v_{\theta}-(1-v) \zeta_{\theta} \\
\Gamma_{g}^{*} & =-v-\alpha(1-v) v_{g}-(1-v) \zeta_{g} .
\end{aligned}
$$

Planning problem. We can again adopt the (IS), (NKPC) and initial condition as they were specified in Section 4 . Therefore, we can write country i's disaggregated planning problem as

$$
\min _{\hat{\theta}^{i},\left\{\left\{\hat{\tau}_{t}^{i,}, \hat{g}_{t}^{i}\right\}_{t \geq 0}\right.} \int_{0}^{\infty} e^{-\rho t}\left[\alpha_{\pi}\left(\hat{\bar{\pi}}_{H, t}^{i}\right)^{2}-\left.\hat{U}_{t}^{i}\right|_{\hat{\theta}_{t}^{i}=\hat{\theta}^{i}}\right] d t,
$$

subject to

$$
\begin{aligned}
\dot{\hat{\bar{y}}}_{t}^{i} & =-D^{\prime}\left(\hat{\bar{\pi}}_{H, t}^{i}+\dot{\tilde{s}}_{t}^{i}\right)+\bar{D} \dot{\hat{\bar{g}}}_{t}^{i}+D \dot{\hat{\bar{\tau}}}_{t}^{i, r} \\
\dot{\hat{\bar{\tau}}}_{H, t}^{i} & =\rho \hat{\bar{\pi}}_{H, t}^{i}-\kappa_{y} \hat{\bar{y}}_{t}^{i}-\kappa_{\theta} \hat{\bar{\theta}}^{i}-\kappa_{\tau} \hat{\bar{\tau}}_{t}^{i, r}-\kappa_{g} \hat{\bar{g}}_{t}^{i}
\end{aligned}
$$




$$
\begin{aligned}
\Lambda_{y} \hat{\bar{y}}_{0}^{i} & =-\Lambda_{s} \tilde{s}_{0}^{i}+\Lambda_{\theta} \hat{\bar{\theta}}^{i}+\Lambda_{\tau} \hat{\bar{\tau}}_{0}^{i, r}+\Lambda_{g} \hat{\bar{g}}_{0}^{i} \\
0 & =\int_{0}^{\infty} e^{-\rho t}\left[\Gamma_{y}^{*} \hat{\bar{y}}_{t}^{i}+\Gamma_{\theta}^{*} \hat{\bar{\theta}}^{i}+\Gamma_{\tau}^{*} \hat{\bar{\tau}}_{t}^{i, r}+\Gamma_{g}^{*} \hat{\bar{g}}_{t}^{i}\right] d t
\end{aligned}
$$

where $D^{\prime}=\frac{\Lambda_{s}}{\Lambda_{y}}, \bar{D}=\frac{\Lambda_{g}}{\Lambda_{y}}$ and $D=\frac{\Lambda_{\tau}}{\Lambda_{y}}$, and

$$
\begin{aligned}
\left.\hat{\bar{U}}_{t}^{i}\right|_{\hat{\theta}_{t}^{i}}=\hat{\bar{\theta}}^{i}= & \alpha_{y y}\left(\hat{\bar{y}}_{t}^{i}\right)^{2}+\alpha_{\theta \theta}\left(\hat{\bar{\theta}}^{i}\right)^{2}+\alpha_{\tau \tau}\left(\hat{\bar{\tau}}_{t}^{i, r}\right)^{2}+\alpha_{g g}\left(\hat{\bar{g}}_{t}^{i}\right)^{2} \\
& +\alpha_{y \theta} \hat{\bar{y}}_{t}^{i} \hat{\bar{\theta}}^{i}+\alpha_{y g} \hat{\bar{y}}_{t}^{i} \hat{\bar{g}}_{t}^{i}+\alpha_{y \tau} \hat{\bar{y}}_{t}^{i} \hat{\bar{\tau}}_{t}^{i, r}+\alpha_{g \theta} \hat{\bar{\theta}}_{t}^{i} \hat{\bar{\theta}}^{i}+\alpha_{g \tau} \hat{\bar{g}}_{t}^{i} \hat{\bar{\tau}}_{t}^{i, r}+\alpha_{\tau \theta} \hat{\bar{\tau}}_{t}^{i, r} \hat{\bar{\theta}}^{i} .
\end{aligned}
$$

Control problem. As before, we incorporate the initial condition and the country budget constraint into the objective function using Lagrange multipliers $\Delta$ and $\lambda$, respectively. Furthermore, we replace the time derivatives $\dot{\hat{g}}_{t}^{i}$ and $\dot{\hat{\tau}}_{t}^{i, r}$ in the (IS) equation with $\hat{\bar{\omega}}_{t}^{i}$ and $\hat{\bar{v}}_{t}^{i}$, respectively, to recover the standard optimal control problem structure. We add these equations as dynamic state equations. We can now write down the Hamiltonian associated with this control problem. We have

$$
\begin{aligned}
H\left(x_{t}^{i}, u_{t}^{i}, \mu_{t}^{i}\right)= & \alpha_{\pi}\left(\hat{\bar{\pi}}_{H, t}^{i}\right)^{2}-\left.\hat{\bar{U}}_{t}^{i}\right|_{\hat{\theta}_{t}^{i}=\hat{\theta}^{i}} \\
& +\Delta\left[\Lambda_{y} \hat{\bar{y}}_{0}^{i}+\Lambda_{s} \tilde{s}_{0}^{i}-\Lambda_{g} \hat{\bar{g}}_{0}^{i}-\Lambda_{\theta} \hat{\bar{\theta}}^{i}-\Lambda_{\tau} \hat{\bar{\tau}}_{0}^{i, r}\right] \\
& +\lambda\left[\Gamma_{y}^{*} \hat{\bar{y}}_{t}^{i}+\Gamma_{g}^{*} \hat{\bar{g}}_{t}^{i}+\Gamma_{\tau}^{*} \hat{\bar{\tau}}_{t}^{i, r}+\Gamma_{\theta}^{*} \hat{\bar{\theta}}^{i}\right] \\
& +\mu_{y, t}^{i}\left[D \hat{\bar{v}}_{t}^{i}+\bar{D} \hat{\bar{\omega}}_{t}^{i}-D^{\prime}\left(\hat{\bar{\pi}}_{H, t}^{i}+\dot{\tilde{s}}_{t}^{i}\right)\right] \\
& +\mu_{\tau, t}^{i} \hat{\bar{v}}_{t}^{i} \\
& +\mu_{g, t}^{i} \hat{\bar{\omega}}_{t}^{i} \\
& +\mu_{\pi, t}^{i}\left[\rho \hat{\bar{\pi}}_{H, t}^{i}-\kappa_{y} \hat{\bar{y}}_{t}^{i}-\kappa_{\theta} \hat{\bar{\theta}}^{i}-\kappa_{\tau} \hat{\bar{\tau}}_{t}^{i, r}-\kappa_{g} \hat{\bar{g}}_{t}^{i}\right],
\end{aligned}
$$

where $x_{t}^{i}=\left\{\hat{\bar{\pi}}_{H, t}^{i}, \hat{\bar{y}}_{t}^{i}, \hat{\bar{\tau}}_{t}^{i, r}, \hat{\bar{g}}_{t}^{i}\right\}$ denotes the vector of state variables, $u_{t}^{i}=\left\{\hat{\bar{v}}_{t}^{i}, \hat{\bar{\omega}}_{t}^{i}\right\}$ the vector of control variables, and $\mu_{t}^{i}=\left\{\mu_{\pi, t}^{i}, \mu_{y, t}^{i}, \mu_{g, t}^{i}, \mu_{\tau, t}^{i}\right\}$ the vector of costates.

\section{D.9.1 Optimality Conditions}

The first-order optimality conditions associated with this problem are given by

\section{Optimality:}

$$
\begin{aligned}
& D \mu_{y, t}^{i}+\mu_{\tau, t}^{i}=0 \\
& \bar{D} \mu_{y, t}^{i}+\mu_{g, t}^{i}=0
\end{aligned}
$$

\section{Multiplier:}




$$
\begin{aligned}
\dot{\mu}_{\pi, t}^{i} & =D^{\prime} \mu_{y, t}^{i}-2 \alpha_{\pi} \hat{\bar{\pi}}_{H, t}^{i} \\
\rho \mu_{y, t}^{i}-\dot{\mu}_{y, t}^{i} & =\Gamma_{y}^{*} \lambda-\kappa_{y} \mu_{\pi, t}^{i}-2 \alpha_{y y} \hat{\bar{y}}_{t}^{i}-\alpha_{y \theta} \hat{\bar{\theta}}^{i}-\alpha_{y g} \hat{\bar{g}}_{t}^{i}-\alpha_{y \tau} \hat{\bar{\tau}}_{t}^{i, r} \\
\rho \mu_{\tau, t}^{i}-\dot{\mu}_{\tau, t}^{i} & =\Gamma_{\tau}^{*} \lambda-\kappa_{\tau} \mu_{\pi, t}^{i}-2 \alpha_{\tau \tau} \hat{\bar{\tau}}_{t}^{i, r}-\alpha_{\tau \theta} \hat{\bar{\theta}}^{i}-\alpha_{y \tau} \hat{\bar{y}}_{t}^{i}-\alpha_{g \tau} \hat{\bar{g}}_{t}^{i} \\
\rho \mu_{g, t}^{i}-\dot{\mu}_{g, t}^{i} & =\Gamma_{g}^{*} \lambda-\kappa_{g} \mu_{\pi, t}^{i}-2 \alpha_{g g} \hat{\bar{g}}_{t}^{i}-\alpha_{y g} \hat{\bar{g}}^{i}-\alpha_{g \theta} \hat{\bar{\theta}}^{i}-\alpha_{g \tau} \hat{\bar{\tau}}_{t}^{i, r}
\end{aligned}
$$

\section{State:}

$$
\begin{aligned}
\dot{\hat{\bar{y}}}_{t}^{i} & =-D^{\prime}\left(\hat{\bar{\pi}}_{H, t}^{i}+\dot{\tilde{s}}_{t}^{i}\right)+D \hat{\bar{v}}_{t}^{i}+\bar{D} \hat{\bar{\omega}}_{t}^{i} \\
\dot{\hat{\bar{\tau}}}_{t}^{i, r} & =\hat{\bar{v}}_{t}^{i} \\
\dot{\overline{\hat{g}}}_{t}^{i, r} & =\hat{\bar{\omega}}_{t}^{i} \\
\dot{\hat{\bar{\pi}}}_{H, t}^{i} & =\rho \hat{\bar{\pi}}_{H, t}^{i}-\kappa_{y} \hat{\bar{y}}_{t}^{i}-\kappa_{g} \hat{\bar{g}}_{t}^{i}-\kappa_{\theta} \hat{\bar{\theta}}^{i}-\kappa_{\tau} \hat{\bar{\tau}}_{t}^{i, r}
\end{aligned}
$$

\section{Initial conditions:}

$$
\begin{aligned}
& \mu_{\pi, 0}^{i}=0 \\
& \mu_{y, 0}^{i}=\Delta \Lambda_{y} \\
& \mu_{\tau, 0}^{i}=-\Delta \Lambda_{\tau} \\
& \mu_{g, 0}^{i}=-\Delta \Lambda_{g} .
\end{aligned}
$$

We can conveniently reduce the dimensionality of this system of differential equations. Following steps analogous to those in earlier sections, we can write

$$
\begin{aligned}
\dot{\hat{\bar{\delta}}}_{t}^{i}= & -\frac{1}{\alpha_{g \tau}+D \alpha_{y g}+\bar{D}\left(2 \alpha_{y y} D+\alpha_{y \tau}\right)}\left[\left(\kappa_{\tau}+D \kappa_{y}\right) D^{\prime} \mu_{y, t}^{i}\right. \\
& -\left(2 \alpha_{\pi}\left(\kappa_{\tau}+D \kappa_{y}\right)+D^{\prime}\left(2 \alpha_{y y} D+\alpha_{y \tau}\right)\right) \hat{\bar{\pi}}_{H, t}^{i}-D^{\prime}\left(2 \alpha_{y y} D+\alpha_{y \tau}\right) \dot{\tilde{s}}_{t}^{i} \\
& \left.+\left(D\left(2 \alpha_{y y} D+\alpha_{y \tau}\right)+2 \alpha_{\tau \tau}+D \alpha_{y \tau}\right) \dot{\hat{\bar{\tau}}}_{t}^{i, r}\right] \\
\equiv & l_{\mu_{y}} \mu_{y, t}^{i}+l_{\pi} \hat{\bar{\pi}}_{H, t}^{i}+l_{s} \dot{\hat{s}}_{t}^{i}+l_{\tau} \dot{\hat{\bar{\tau}}}_{t}^{i, r} .
\end{aligned}
$$

We then find that

$$
\begin{aligned}
\dot{\hat{\tau}}_{t}^{i, r}= & -\frac{1}{2 \alpha_{y y} D \bar{D}+\alpha_{y g} D+\alpha_{g \tau}+\bar{D} \alpha_{y \tau}+l_{\tau}\left(2 \alpha_{y y} \bar{D}^{2}+\alpha_{y g} \bar{D}+2 \alpha_{g g}+\bar{D} \alpha_{y g}\right)} . \\
& {\left[\left(D^{\prime}\left(\kappa_{g}+\bar{D} \kappa_{y}\right)+l_{\mu_{y}}\left(2 \alpha_{y y} \bar{D}^{2}+\alpha_{y g} \bar{D}+2 \alpha_{g g}+\bar{D} \alpha_{y g}\right)\right) \mu_{y, t}^{i}\right.} \\
& +\left(l_{\pi}\left(2 \alpha_{y y} \bar{D}^{2}+\alpha_{y g} \bar{D}+2 \alpha_{g g}+\bar{D} \alpha_{y g}\right)-2 \alpha_{\pi}\left(\kappa_{g}+\bar{D} \kappa_{y}\right)-D^{\prime}\left(2 \alpha_{y y} \bar{D}+\alpha_{y g}\right)\right) \hat{\pi}_{H, t}^{i}
\end{aligned}
$$




$$
\begin{aligned}
& \left.-\left(D^{\prime}\left(2 \alpha_{y y} \bar{D}+\alpha_{y g}\right)+l_{s}\left(2 \alpha_{y y} \bar{D}^{2}+\alpha_{y g} \bar{D}+2 \alpha_{g g}+\bar{D} \alpha_{y g}\right)\right) \dot{\tilde{s}}_{t}^{i}\right] \\
\equiv & H_{\mu_{y}} \mu_{y, t}^{i}+H_{\pi} \hat{\bar{\pi}}_{H, t}^{i}+H_{s} \dot{\tilde{s}}_{t}^{i}
\end{aligned}
$$

and

$$
\dot{\hat{\bar{g}}}_{t}^{i}=l_{\mu_{y}} \mu_{y, t}^{i}+l_{\pi} \hat{\bar{\pi}}_{H, t}^{i}+l_{s} \dot{\tilde{s}}_{t}^{i}+l_{\tau}\left(H_{\mu_{y}} \mu_{y, t}^{i}+H_{\pi} \hat{\bar{\pi}}_{H, t}^{i}+H_{s} \dot{\tilde{s}}_{t}^{i}\right) .
$$

Using the (IS) equation, we have

$$
\dot{\hat{y}}_{t}^{i}=\left(D H_{\mu_{y}}+\bar{D} L_{\mu_{y}}\right) \mu_{y, t}^{i}+\left(D H_{\pi}+\bar{D} L_{\pi}-D^{\prime}\right) \hat{\bar{\pi}}_{H, t}^{i}+\left(D H_{s}+\bar{D} L_{s}+D^{\prime}\right) \dot{\tilde{s}}_{t}^{i} .
$$

Finally, we can write

$$
\begin{aligned}
\dot{\mu}_{y, t}^{i}= & \rho \mu_{y, t}^{i}+\lambda \frac{\kappa_{y} \Gamma_{g}^{*}-\kappa_{g} \Gamma_{y}^{*}}{\kappa_{g}+\bar{D} \kappa_{y}}+\hat{y}_{t}^{i} \frac{2 \alpha_{y y} \kappa_{g}-\alpha_{y g} \kappa_{y}}{\kappa_{g}+\bar{D} \kappa_{y}}+\hat{g}_{t}^{i} \frac{\alpha_{y g} \kappa_{g}-2 \alpha_{g g} \kappa_{y}}{\kappa_{g}+\bar{D} \kappa_{y}} \\
& +\hat{\bar{\tau}}_{t}^{i, r} \frac{\alpha_{y \tau} \kappa_{g}-\alpha_{g \tau} \kappa_{y}}{\kappa_{g}+\bar{D} \kappa_{y}}+\hat{\theta}^{i} \frac{\alpha_{y \theta} \kappa_{g}-\alpha_{g \theta} \kappa_{y}}{\kappa_{g}+\bar{D} \kappa_{y}} .
\end{aligned}
$$

Summarizing, we obtain the system

$$
\begin{aligned}
\dot{\bar{\pi}}_{H, t}^{i} & =\rho \hat{\bar{\pi}}_{H, t}^{i}-\kappa_{y} \hat{\bar{y}}_{t}^{i}-\kappa_{g} \hat{\bar{g}}_{t}^{i}-\kappa_{\theta} \hat{\theta}^{i}-\kappa_{\tau} \hat{\bar{\tau}}_{t}^{i, r} \\
\dot{\hat{y}}_{t}^{i} & \equiv J_{\mu_{y}} \mu_{y, t}^{i}+J_{\pi} \hat{\bar{\pi}}_{H, t}^{i}+J_{s} \dot{\tilde{s}}_{t}^{i} \\
\dot{\bar{\tau}}_{t}^{i, r} & =H_{\mu_{y}} \mu_{y, t}^{i}+H_{\pi} \hat{\bar{\pi}}_{H, t}^{i}+H_{s} \dot{\tilde{s}}_{t}^{i} \\
\dot{\hat{g}}_{t}^{i} & \equiv L_{\mu_{y}} \mu_{y, t}^{i}+L_{\pi} \hat{\bar{\pi}}_{H, t}^{i}+L_{s} \dot{\tilde{s}}_{t}^{i} \\
\dot{\mu}_{y, t}^{i} & \equiv \rho \mu_{y, t}^{i}+K_{\lambda} \lambda+K_{y} \hat{\bar{y}}_{t}^{i}+K_{g} \hat{\bar{g}}_{t}^{i}+K_{\tau} \hat{\bar{\tau}}_{t}^{i, r}+K_{\theta} \hat{\bar{\theta}}^{i} .
\end{aligned}
$$

\section{D.9.2 A Targeting Rule for Government Spending}

If we let $X_{t}^{i}=\left\{\hat{\bar{\pi}}_{H, t}^{i}, \hat{\bar{y}}_{t}^{i}, \hat{\bar{\tau}}_{t}^{i, r}, \hat{\hat{g}}_{t}^{i}, \mu_{y, t}^{i}\right\}$, then the dynamical system of optimality conditions can be written as

$$
\dot{X}_{t}^{i}=A X_{t}^{i}+B_{t}^{i}
$$

where

$$
A=\left(\begin{array}{ccccc}
\rho & -\kappa_{y} & -\kappa_{\tau} & -\kappa_{g} & 0 \\
J_{\pi} & 0 & 0 & 0 & J_{\mu_{y}} \\
H_{\pi} & 0 & 0 & 0 & H_{\mu_{y}} \\
L_{\pi} & 0 & 0 & 0 & L_{\mu_{y}} \\
0 & K_{y} & K_{\tau} & K_{g} & \rho
\end{array}\right), \quad B_{t}^{i}=\left(\begin{array}{c}
-\kappa_{\theta} \hat{\theta}^{i} \\
\bar{J}_{t}^{i} \\
\bar{H}_{t}^{i} \\
\bar{L}_{t}^{i} \\
\bar{K}_{t}^{i}
\end{array}\right), \quad X_{0}^{i}=\left(\begin{array}{c}
\hat{\bar{\pi}}_{H, 0}^{i} \\
\hat{y}_{0}^{i} \\
\hat{\bar{\tau}}_{0}^{i, r} \\
\hat{\bar{g}}_{0}^{i} \\
\Delta \Lambda_{y}
\end{array}\right),
$$

and

$$
\bar{J}_{t}^{i}=J_{s} \dot{\tilde{s}}_{t}^{i}
$$




$$
\begin{aligned}
\bar{H}_{t}^{i} & =H_{s} \dot{\tilde{S}}_{t}^{i} \\
\bar{L}_{t}^{i} & =L_{s} \dot{\tilde{S}}_{t}^{i} \\
\bar{K}_{t}^{i} & =K_{\theta} \hat{\theta}^{i}+K_{\lambda} \lambda .
\end{aligned}
$$

The coefficient matrix $A$ has one 0-eigenvalue. This implies that one of the differential equations can be expressed as a function of the other variables in $X_{t}^{i}$. We will exploit this to derive an explicit targeting rule for government spending using tools from linear algebra. We could also prove this result directly starting from the system (292) - (296).

If $A$ has a 0 -eigenvalue, then so does its transpose $A^{\prime}$. Hence, there exists a vector $E$ such that $A^{\prime} E=0$, which gives us $E^{\prime} A=0$. That is, $E$ is the eigenvector associated with the 0 -eigenvalue of the transpose matrix $A^{\prime}$. We have

$$
E^{\prime} \dot{X}_{t}^{i}=E^{\prime} A X_{t}^{i}+E^{\prime} B_{t}^{i}=E^{\prime} B_{t}^{i}
$$

Integrating, we find

$$
E^{\prime} X_{t}^{i}=E^{\prime} X_{0}^{i}+\int_{0}^{t} E^{\prime} B_{s}^{i} d s
$$

It is easy to verify that $E(1)=E(5)=0$. Therefore, we can write

$$
E_{g} \hat{\bar{g}}_{t}^{i}=-E_{y} \hat{\bar{y}}_{t}^{i}-E_{\tau} \hat{\bar{\tau}}_{t}^{i, r}+E^{\prime} X_{0}^{i}+\int_{0}^{t} E^{\prime} B_{s}^{i} d s,
$$

where $E_{y}=E(2), E_{\tau}=E(3)$ and $E_{g}=E(4)$. Let

$$
W=-\psi E_{y} J_{s} a_{0}^{i}-\psi E_{\tau} H_{s} a_{0}^{i}-\psi E_{g} L_{s} a_{0}^{i} .
$$

It then follows from (298) that government spending follows the policy targeting rule

$$
\hat{\bar{g}}_{t}^{i}=-\frac{E_{y}}{E_{g}}\left(\hat{\bar{y}}_{t}^{i}-\hat{\bar{y}}_{0}^{i}\right)-\frac{E_{\tau}}{E_{g}}\left(\hat{\bar{\tau}}_{t}^{i, r}-\hat{\bar{\tau}}_{0}^{i, r}\right)+\hat{\bar{g}}_{0}^{i}+\frac{W}{\psi E_{g}}\left(1-e^{-\psi t}\right) .
$$

So far, we have only shown that the targeting rule holds for some family of eigenvectors $E$. However, we can even solve for $E_{y}, E_{\tau}$ and $E_{g}$ in closed form. Differentiating the targeting rule with respect to time, we have

$$
\dot{\hat{\bar{g}}}_{t}^{i}=-\frac{E_{y}}{E_{g}} \dot{\hat{y}}_{t}^{i}-\frac{E_{\tau}}{E_{g}} \dot{\bar{\tau}}_{t}^{i, r}+\frac{W}{E_{g}} e^{-\psi t} .
$$

Using the original system of equations (292) - (296) to substitute in for $\dot{\hat{\hat{y}}}_{t}^{i}$ and $\dot{\hat{\tau}}_{t}^{i, r}$ yields, after some algebra, the two equations

$$
\begin{aligned}
E_{g} L_{\mu_{y}} & =-E_{y} J_{\mu_{y}}-E_{\tau} H_{\mu_{y}} \\
E_{g} L_{\pi} & =-E_{y} J_{\pi}-E_{\tau} H_{\pi},
\end{aligned}
$$


in the three unknowns $E_{y}, E_{\tau}$ and $E_{g}$. By construction, an uncountably large class of linearly dependent eigenvectors $E$ can give rise to the targeting rule (note that the coefficients in the policy rule are ratios). Therefore, we can normalize $E_{g}=1$, and solve for $E_{y}$ and $E_{\tau}$ :

$$
\begin{aligned}
& E_{y}=-\frac{H_{\pi} L_{\mu_{y}}+H_{\mu_{y}} L_{\pi}}{H_{\pi} J_{\mu_{y}}+H_{\mu_{y}} J_{\pi}} \\
& E_{\tau}=\frac{J_{\pi}}{H_{\pi}} \frac{H_{\pi} L_{\mu_{y}}+H_{\mu_{y}} L_{\pi}}{H_{\pi} J_{\mu_{y}}+H_{\mu_{y}}-\frac{L_{\pi}}{H_{\pi}}} .
\end{aligned}
$$

\section{D.9.3 Solving the Dynamic System}

We can use the targeting rule to simplify the dynamical system of optimality conditions. We have

$$
\begin{aligned}
\dot{\bar{\pi}}_{H, t}^{i} & =\rho \hat{\bar{\pi}}_{H, t}^{i}-\tilde{\kappa}_{y} \hat{\bar{y}}_{t}^{i}-\tilde{\kappa}_{\tau} \hat{\bar{\tau}}_{t}^{i, r}-\kappa_{\theta} \hat{\bar{\theta}}^{i}-\frac{\kappa_{g}}{E_{g}}\left(E_{y} \hat{\bar{y}}_{0}^{i}+E_{\tau} \hat{\bar{\tau}}_{0}^{i, r}\right)-\kappa_{g} \hat{\bar{g}}_{0}^{i}-\kappa_{g} \frac{W}{\psi E_{g}}\left(1-e^{-\psi t}\right) \\
\dot{\hat{y}}_{t}^{i} & \equiv J_{\mu_{y}} \mu_{y, t}^{i}+J_{\pi} \hat{\bar{\pi}}_{H, t}^{i}+\bar{J}_{t}^{i} \\
\dot{\bar{\tau}}_{t}^{i, r} & =H_{\mu_{y}} \mu_{y, t}^{i}+H_{\pi} \hat{\bar{\pi}}_{H, t}^{i}+\bar{H}_{t}^{i} \\
\dot{\mu}_{y, t}^{i} & =\rho \mu_{y, t}^{i}+\tilde{K}_{y} \hat{\bar{y}}_{t}^{i}+\tilde{\bar{K}}_{\tau} \hat{\bar{\tau}}_{t}^{i, r}+\bar{K}_{t}^{i}+\frac{K_{g}}{E_{g}}\left(E_{y} \hat{\bar{y}}_{0}^{i}+E_{\tau} \hat{\bar{\tau}}_{0}^{i, r}\right)+K_{g} \hat{\bar{g}}_{0}^{i}+K_{g} \frac{W}{\psi E_{g}}\left(1-e^{-\psi t}\right),
\end{aligned}
$$

where

$$
\begin{aligned}
& \tilde{\widetilde{\kappa}}_{y}=\kappa_{y}-\kappa_{g} \frac{E_{y}}{E_{g}} \\
& \tilde{\kappa}_{\tau}=\kappa_{\tau}-\kappa_{g} \frac{E_{\tau}}{E_{g}} \\
& \tilde{K}_{y}=K_{y}-K_{g} \frac{E_{y}}{E_{g}} \\
& \tilde{K}_{\tau}=K_{\tau}-K_{g} \frac{E_{\tau}}{E_{g}} .
\end{aligned}
$$

Next, we need to solve for $\hat{\bar{y}}_{0}^{i}$ and $\hat{\bar{\tau}}_{0}^{i, r}$ using the initial conditions

$$
\begin{aligned}
\Lambda_{y} \hat{\bar{y}}_{0}^{i}= & -\Lambda_{s} \tilde{s}_{0}^{i}+\Lambda_{\theta} \hat{\bar{\theta}}^{i}+\Lambda_{\tau} \hat{\bar{\tau}}_{0}^{i, r}+\Lambda_{g} \hat{\bar{g}}_{0}^{i} \\
0= & \lambda\left(\Gamma_{g}^{*}+\bar{D} \Gamma_{y}^{*}\right)-\left(2 \alpha_{g g}+\bar{D} \alpha_{y g}\right) \hat{\bar{g}}_{0}^{i}-\left(2 \alpha_{y y} \bar{D}+\alpha_{y g}\right) \hat{\bar{y}}_{0}^{i} \\
& -\left(\alpha_{g \tau}+\bar{D} \alpha_{y \tau}\right) \hat{\bar{\tau}}_{0}^{i, r}-\left(\alpha_{g \theta}+\bar{D} \alpha_{y \theta}\right) \hat{\bar{\theta}}^{i} .
\end{aligned}
$$

Rearranging and solving the two equations in the two unknowns, we can write

$$
\begin{aligned}
\hat{\bar{\tau}}_{0}^{i, r} & \equiv Q_{\lambda} \lambda+Q_{g} \hat{\bar{g}}_{0}^{i}+Q_{\theta} \hat{\bar{\theta}}^{i}+Q_{s} \tilde{s}_{0}^{i} \\
\hat{\bar{y}}_{0}^{i} & \equiv P_{\lambda} \lambda+P_{g} \hat{\bar{g}}_{0}^{i}+P_{\theta} \hat{\bar{\theta}}^{i}+P_{s} \tilde{s}_{0}^{i} .
\end{aligned}
$$


Hence, we have solved for $\hat{y}_{0}^{i}$ and $\hat{\tau}_{0}^{i, r}$ as functions of the Lagrange multiplier $\lambda, \hat{g}_{0}^{i}$ and $\hat{\theta}^{i}$. Recall that in previous sections, we have solved for the optimal allocation as a function of $\hat{\bar{\theta}}^{i}$. Now, even though the path of government spending is tied down by the targeting rule, $\hat{\bar{g}}_{0}^{i}$ is a free parameter that the social planner can choose optimally. Therefore, we will now go on to solve the optimal allocation as a function of $\left(\hat{\bar{g}}_{0}^{i}, \hat{\bar{\theta}}^{i}\right)$ and then numerically pin down these two free constants.

The transformed dynamical system can now be written in terms of $\tilde{X}_{t}^{i}=\left\{\hat{\bar{\pi}}_{H, t}^{i} \hat{\bar{y}}_{t}^{i}, \hat{\bar{\tau}}_{t}^{i, r}, \mu_{y, t}^{i}\right\}$ as

$$
\dot{X}_{t}^{i}=\tilde{A} \tilde{X}_{t}^{i}+\tilde{B}_{t}^{i}
$$

where

$$
\tilde{A}=\left(\begin{array}{cccc}
\rho & -\tilde{\kappa}_{y} & -\tilde{\bar{\kappa}}_{\tau} & 0 \\
J_{\pi} & 0 & 0 & J_{\mu_{y}} \\
H_{\pi} & 0 & 0 & H_{\mu_{y}} \\
0 & \tilde{K}_{y} & \tilde{K}_{\tau} & \rho
\end{array}\right), \quad \tilde{B}_{t}^{i}=\left(\begin{array}{c}
\kappa_{t}^{i} \\
\bar{J}_{t}^{i} \\
\bar{H}_{t}^{i} \\
K_{t}^{i}
\end{array}\right)
$$

and

$$
\tilde{X}_{0}^{i}=\left(\begin{array}{c}
\hat{\bar{\pi}}_{H, 0}^{i} \\
P_{\lambda} \lambda+P_{g} \hat{\bar{g}}_{0}^{i}+P_{\theta} \hat{\bar{\theta}}^{i}+P_{s} \tilde{s}_{0}^{i} \\
Q_{\lambda} \lambda+Q_{g} \hat{\bar{g}}_{0}^{i}+Q_{\theta} \hat{\bar{\theta}}^{i}+Q_{s} \tilde{s}_{0}^{i} \\
\Delta \Lambda_{y}
\end{array}\right),
$$

where

$$
\begin{aligned}
\kappa_{t}^{i}= & -\kappa_{\theta} \hat{\bar{\theta}}^{i}-\kappa_{g} \frac{W}{\psi E_{g}}\left(1-e^{-\psi t}\right)-\lambda \frac{\kappa_{g}}{E_{g}}\left(E_{y} P_{\lambda}+E_{\tau} Q_{\lambda}\right) \\
& -\hat{\bar{g}}_{0}^{i} \frac{\kappa_{g}}{E_{g}}\left(E_{y} P_{g}+E_{\tau} Q_{g}+E_{g}\right)-\hat{\bar{\theta}}^{i} \frac{\kappa_{g}}{E_{g}}\left(E_{y} P_{\theta}+E_{\tau} Q_{\theta}\right) \\
& -\tilde{s}_{0}^{i} \frac{\kappa_{g}}{E_{g}}\left(E_{y} P_{s}+E_{\tau} Q_{s}\right) \\
\equiv & -\tilde{\kappa}_{\theta} \hat{\bar{\theta}}^{i}-\tilde{\kappa}_{\lambda} \lambda-\tilde{\kappa}_{g} \hat{\bar{g}}_{0}^{i}-\tilde{\kappa}+\kappa_{g} \frac{W}{\psi E_{g}} e^{-\psi t}
\end{aligned}
$$

with

$$
\tilde{\tilde{\kappa}}=\tilde{s}_{0}^{i} \frac{\kappa_{g}}{E_{g}}\left(E_{y} P_{s}+E_{\tau} Q_{s}\right)+\kappa_{g} \frac{W}{\psi E_{g}}
$$

and

$$
\begin{aligned}
K_{t}^{i}= & K_{g} \frac{W}{\psi E_{g}}\left(1-e^{-\psi t}\right)+\lambda \frac{K_{g}}{E_{g}}\left(E_{y} P_{\lambda}+E_{\tau} Q_{\lambda}\right) \\
& +\hat{\bar{g}}_{0}^{i} \frac{K_{g}}{E_{g}}\left(E_{y} P_{g}+E_{\tau} Q_{g}\right)+\hat{\bar{\theta}}^{i} \frac{K_{g}}{E_{g}}\left(E_{y} P_{\theta}+E_{\tau} Q_{\theta}\right) \\
& +\tilde{s}_{0}^{i} \frac{K_{g}}{E_{g}}\left(E_{y} P_{s}+E_{\tau} Q_{s}\right)+K_{\lambda} \lambda+K_{\theta} \hat{\theta}^{i} \\
\equiv & \tilde{\bar{K}}_{\lambda} \lambda+\tilde{K}_{g} \hat{\bar{g}}_{0}^{i}+\tilde{\bar{K}}_{\theta} \hat{\theta}^{i}+\tilde{K}-K_{g} \frac{W}{\psi E_{g}} e^{-\psi t}
\end{aligned}
$$


with

$$
\tilde{K}=\tilde{s}_{0}^{i} \frac{K_{g}}{E_{g}}\left(E_{y} P_{s}+E_{\tau} Q_{s}\right)+K_{g} \frac{W}{\psi E_{g}} .
$$

Therefore, we can write the forcing term vector as

$$
\tilde{B}_{t}^{i}=\left(\begin{array}{c}
-\tilde{\tilde{\kappa}}_{\theta} \hat{\theta}^{i}-\tilde{\bar{\kappa}}_{\lambda} \lambda-\tilde{\kappa}_{g} \hat{\bar{g}}_{0}^{i}-\tilde{\kappa}+\kappa_{g} \frac{W}{\psi E_{g}} e^{-\psi t} \\
\bar{J}_{t}^{i} \\
\bar{H}_{t}^{i} \\
\tilde{K}_{\lambda} \lambda+\tilde{K}_{g} \hat{\bar{g}}_{0}^{i}+\tilde{K}_{\theta} \hat{\bar{\theta}}^{i}+\tilde{\bar{K}}-K_{g} \frac{W}{\psi E_{g}} e^{-\psi t}
\end{array}\right) .
$$

The solution is then given by

$$
\tilde{X}_{t}^{i}=e^{\tilde{A} t}\left[\tilde{X}_{0}^{i}+\int_{0}^{t} e^{-\tilde{A} s} \tilde{B}_{s}^{i} d s\right] .
$$

Using the same steps as in previous sections, we can manipulate this solution to obtain

$$
\begin{aligned}
& X_{t}^{i}=e^{\tilde{A} t}\left[\tilde{X}_{0}^{i}-\left(\tilde{\tilde{\kappa}}_{\theta} \hat{\theta}^{i}+\tilde{\tilde{\kappa}}_{\lambda} \lambda+\tilde{\kappa}_{g} \hat{\bar{g}}_{0}^{i}+\tilde{\tilde{\kappa}}\right) \tilde{A}^{-1} E_{1}+\left(\tilde{K}_{\theta} \hat{\theta}^{i}+\tilde{K}_{\lambda} \lambda+\tilde{K}_{g} \hat{\bar{g}}_{0}^{i}+\tilde{K}\right) \tilde{A}^{-1} E_{4}+(\tilde{A}+\psi I)^{-1} \hat{\bar{E}}\right] \\
& +\left(\tilde{\tilde{K}}_{\theta} \hat{\theta}^{i}+\tilde{\kappa}_{\lambda} \lambda+\tilde{\kappa}_{g} \hat{\bar{g}}_{0}^{i}+\tilde{\kappa}\right) \tilde{A}^{-1} E_{1}-\left(\tilde{K}_{\theta} \hat{\bar{\theta}}^{i}+\tilde{K}_{\lambda} \lambda+\tilde{K}_{g} \hat{\bar{g}}_{0}^{i}+\tilde{\tilde{K}}\right) \tilde{A}^{-1} E_{4}-(\tilde{A}+\psi I)^{-1} e^{-\psi t} \hat{\bar{E}},
\end{aligned}
$$

where

$$
\hat{\bar{E}}=W_{2} E_{2}+W_{3} E_{3}-K_{g} \frac{W}{\psi E_{g}} E_{4}+\kappa_{g} \frac{W}{\psi E_{g}} E_{1}
$$

and $W_{2}$ and $W_{3}$ are defined as in previous sections.

\section{D.9.4 Stability}

As before, we require the term in square brackets to be spanned in the stable manifold to guarantee the stability of the system. $\tilde{A}$ again has two negative eigenvalues. Let $V_{j}, j \in\{1,2\}$ denote the eigenvectors of $A$ associated with these negative eigenvalues, which we denote $\lambda_{1}$ and $\lambda_{2}$. The spanning condition can then be written as

$0=\alpha_{1} V_{1}+\alpha_{2} V_{2} \tilde{X}_{0}^{i}-\left(\tilde{\tilde{\kappa}}_{\theta} \hat{\bar{\theta}}^{i}+\tilde{\tilde{\kappa}}_{\lambda} \lambda+\tilde{\kappa}_{g} \hat{\bar{g}}_{0}^{i}+\tilde{\bar{\kappa}}\right) \tilde{A}^{-1} E_{1}+\left(\tilde{\bar{K}}_{\theta} \hat{\bar{\theta}}^{i}+\tilde{K}_{\lambda} \lambda+\tilde{K}_{g} \hat{\bar{g}}_{0}^{i}+\tilde{\bar{K}}\right) \tilde{A}^{-1} E_{4}+(\tilde{A}+\psi I)^{-1} \hat{\bar{E}}$

for some $\alpha_{j} \in \mathbb{C}, j \in\{1,2\}$.

The solution, including the above stability condition, is now given as a function of the free parameters $\left(\hat{\bar{g}}_{0}^{i}, \hat{\bar{\theta}}^{i}\right)$ and $Z=\left(\alpha_{1}, \alpha_{2}, \lambda, \Delta, \hat{\bar{\pi}}_{H, 0}^{i}\right)$. We can pin down the latter set of free parameters by imposing the stability condition as well as the country budget constraint. The stability condition yields a system of four equations if we premultiply both sides by the projection matrices $E_{i}, i \in\{1,2,3,4\}$. Unlike in previous sections, we have already solved for and substituted in $\hat{y}_{0}^{i}$ and $\hat{\bar{\tau}}_{0}^{i, r}$ using the two 
initial conditions.

We now substitute the target rule for government spending into the country budget constraint

$$
\begin{aligned}
0= & \int_{0}^{\infty} e^{-\rho t}\left[\Gamma_{y}^{*} \hat{\bar{y}}_{t}^{i}+\Gamma_{\theta}^{*} \hat{\bar{\theta}}^{i}+\Gamma_{\tau}^{*} \hat{\bar{\tau}}_{t}^{i, r}+\Gamma_{g}^{*} \hat{\bar{g}}_{t}^{i}\right] d t \\
= & \frac{\Gamma_{\theta}^{*}}{\rho} \hat{\bar{\theta}}^{i}+\frac{\Gamma_{g}^{*}}{\rho} \hat{\bar{g}}_{0}^{i}+\frac{\Gamma_{g}^{*}}{\rho E_{g}}\left(E_{y} \hat{\bar{y}}_{0}^{i}+E_{\tau} \hat{\bar{\tau}}_{0}^{i, r}\right)+\Gamma_{g}^{*} \frac{W}{\rho \psi E_{g}} \\
& \left.+\int_{0}^{\infty} e^{-\rho t}\left[\left(\Gamma_{y}^{*}-\Gamma_{g}^{*} \frac{E_{y}}{E_{g}}\right) \hat{\bar{y}}_{t}^{i}+\left(\Gamma_{\tau}^{*}-\Gamma_{g}^{*} \frac{E_{\tau}}{E_{g}}\right) \hat{\bar{\tau}}_{t}^{i, r}-\Gamma_{g}^{*} \frac{W}{\psi E_{g}} e^{-\psi t}\right)\right] d t \\
= & \frac{\Gamma_{\theta}^{*}}{\rho} \hat{\bar{\theta}}^{i}+\frac{\Gamma_{g}^{*}}{\rho} \hat{\bar{g}}_{0}^{i}+\frac{\Gamma_{g}^{*}}{\rho E_{g}} E_{y}\left(P_{\lambda} \lambda+P_{g} \hat{\bar{g}}_{0}^{i}+P_{\theta} \hat{\bar{\theta}}^{i}+P_{s} \tilde{s}_{0}^{i}\right) \\
& +\frac{\Gamma_{g}^{*}}{\rho E_{g}} E_{\tau}\left(Q_{\lambda} \lambda+Q_{g} \hat{\bar{g}}_{0}^{i}+Q_{\theta} \hat{\bar{\theta}}^{i}+Q_{s} \tilde{s}_{0}^{i}\right)+\Gamma_{g}^{*} \frac{W}{\rho \psi E_{g}}-\Gamma_{g}^{*} \frac{W}{(\rho+\psi) \psi E_{g}}+\int_{0}^{\infty} e^{-\rho t} \tilde{\bar{E}}^{\prime} \tilde{X}_{t}^{i} d t
\end{aligned}
$$

where

$$
\tilde{\bar{E}}=\left(\Gamma_{y}^{*}-\Gamma_{g}^{*} \frac{E_{y}}{E_{g}}\right) E_{2}+\left(\Gamma_{\tau}^{*}-\Gamma_{g}^{*} \frac{E_{\tau}}{E_{g}}\right) E_{3} .
$$

We have

$$
\begin{aligned}
\int_{0}^{\infty} e^{-\rho t} \tilde{\bar{E}}^{\prime} \tilde{X}_{t}^{i} d t= & \frac{\alpha}{\lambda_{1}-\rho} \tilde{\bar{E}}^{\prime} V_{1}+\frac{\alpha_{2}}{\lambda_{2}-\rho} \tilde{\bar{E}}^{\prime} V_{2}+\frac{1}{\rho}\left(\tilde{\bar{\kappa}}_{\theta} \hat{\bar{\theta}}^{i}+\tilde{\tilde{\kappa}}_{\lambda} \lambda+\tilde{\tilde{\kappa}}_{g} \hat{\bar{g}}_{0}^{i}+\tilde{\tilde{\bar{\kappa}}}\right) \tilde{\bar{E}}^{\prime} \tilde{A}^{-1} E_{1} \\
& -\frac{1}{\rho}\left(\tilde{\bar{K}}_{\theta} \hat{\bar{\theta}}^{i}+\tilde{\bar{K}}_{\lambda} \lambda+\tilde{\bar{K}}_{g} \hat{\bar{g}}_{0}^{i}+\tilde{\tilde{K}}\right) \tilde{\bar{E}}^{\prime} \tilde{A}^{-1} E_{4}-\frac{1}{\rho+\psi} \tilde{\bar{E}}^{\prime}(\tilde{A}+\psi I)^{-1} \hat{\bar{E}}
\end{aligned}
$$

Therefore, putting everything together and grouping terms, the country budget constraint becomes

$$
\begin{aligned}
0= & \hat{\bar{\theta}}^{i}\left(\frac{\Gamma_{\theta}^{*}}{\rho}+\frac{\Gamma_{g}^{*}}{\rho E_{g}}\left(E_{y} P_{\theta}+E_{\tau} Q_{\theta}\right)\right)+\Gamma_{g}^{*} \frac{W}{\rho(\rho+\psi) E_{g}}+\lambda \frac{\Gamma_{g}^{*}}{\rho E_{g}}\left(E_{y} P_{\lambda}+E_{\tau} Q_{\lambda}\right) \\
& +\hat{\bar{g}}_{0}^{i}\left(\frac{\Gamma_{g}^{*}}{\rho}+\frac{\Gamma_{g}^{*}}{\rho E_{g}}\left(E_{y} P_{g}+E_{\tau} Q_{g}\right)\right)+\tilde{s}_{0}^{i} \frac{\Gamma_{g}^{*}}{\rho E_{g}}\left(E_{y} P_{s}+E_{\tau} Q_{s}\right) \\
& +\frac{\alpha_{1}}{\lambda_{1}-\rho} \tilde{\bar{E}}^{\prime} V_{1}+\frac{\alpha_{2}}{\lambda_{2}-\rho} \tilde{\bar{E}}^{\prime} V_{2}+\frac{1}{\rho}\left(\tilde{\bar{\kappa}}_{\theta} \hat{\bar{\theta}}^{i}+\tilde{\bar{\kappa}}_{\lambda} \lambda+\tilde{\bar{\kappa}}_{g} \hat{\bar{g}}_{0}^{i}+\tilde{\bar{\kappa}}^{\prime}\right) \tilde{\bar{E}}^{\prime} \tilde{A}^{-1} E_{1} \\
& -\frac{1}{\rho}\left(\tilde{\bar{K}}_{\theta} \hat{\bar{\theta}}^{i}+\tilde{\bar{K}}_{\lambda} \lambda+\tilde{\bar{K}}_{g} \hat{\bar{g}}_{0}^{i}+\tilde{\bar{K}}\right) \tilde{\bar{E}}^{\prime} \tilde{A}^{-1} E_{4}-\frac{1}{\rho+\psi} \tilde{\bar{E}}^{\prime}(\tilde{A}+\psi I)^{-1} \hat{\bar{E}} .
\end{aligned}
$$

Together, the stability condition and the country budget constraint imply a system of equations $M Z=N$, with

$$
M=\left(\begin{array}{ccccc}
E_{1}^{\prime} V_{1} & E_{1}^{\prime} V_{2} & -\tilde{\bar{\kappa}}_{\lambda} E_{1}^{\prime} A^{-1} E_{1}+\tilde{\bar{K}}_{\lambda} E_{1}^{\prime} A^{-1} E_{4} & 0 & 1 \\
E_{2}^{\prime} V_{1} & E_{2}^{\prime} V_{2} & P_{\lambda}-\tilde{\bar{\kappa}}_{\lambda} E_{2}^{\prime} A^{-1} E_{1}+\tilde{K}_{\lambda} E_{2}^{\prime} A^{-1} E_{4} & 0 & 0 \\
E_{3}^{\prime} V_{1} & E_{3}^{\prime} V_{2} & Q_{\lambda}-\tilde{\bar{\kappa}}_{\lambda} E_{3}^{\prime} A^{-1} E_{1}+\tilde{\bar{K}}_{\lambda} E_{3}^{\prime} A^{-1} E_{4} & 0 & 0 \\
E_{4}^{\prime} V_{1} & E_{4}^{\prime} V_{2} & -\tilde{\tilde{\kappa}}_{\lambda} E_{4}^{\prime} A^{-1} E_{1}+\tilde{\bar{K}}_{\lambda} E_{4}^{\prime} A^{-1} E_{4} & \Lambda_{y} & 0 \\
\frac{\tilde{E}^{\prime}}{\lambda_{1}-\rho} V_{1} & \frac{\tilde{E}^{\prime}}{\lambda_{2}-\rho} V_{2} & \frac{\Gamma_{g}^{*}}{\rho E_{g}}\left(E_{y} P_{\lambda}+E_{\tau} Q_{\lambda}\right)+\frac{\tilde{\kappa}_{\lambda}}{\rho} \tilde{\bar{E}}^{\prime} \tilde{A}^{-1} E_{1}-\frac{\tilde{K}_{\lambda}}{\rho} \tilde{\bar{E}}^{\prime} \tilde{A}^{-1} E_{4} & 0 & 0
\end{array}\right)
$$


and

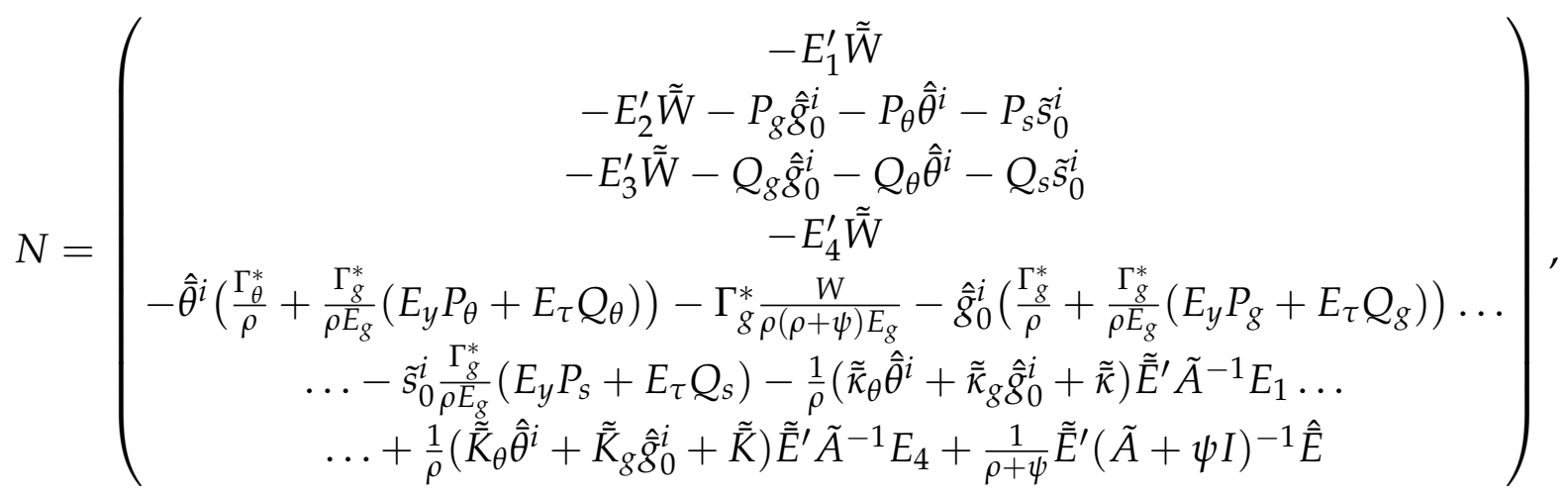

where $\tilde{\bar{W}}=-\left(\tilde{\tilde{K}}_{\theta} \hat{\bar{\theta}}^{i}+\tilde{\kappa}_{g} \hat{\bar{g}}_{0}^{i}+\tilde{\tilde{\kappa}}\right) \tilde{A}^{-1} E_{1}+\left(\tilde{K}_{\theta} \hat{\bar{\theta}}^{i}+\tilde{K}_{g} \hat{\bar{g}}_{0}^{i}+\tilde{K}\right) \tilde{A}^{-1} E_{4}+(\tilde{A}+\psi I)^{-1} \hat{\bar{E}}$.

Inverting $M$, which is non-singular in all of our calibrations, we can solve for $Z$ numerically. Given $Z$, we have the full solution of the optimal allocation as a function of the two remaining free parameters $\left(\hat{\bar{g}}_{0}^{i}, \hat{\bar{\theta}}^{i}\right)$, over which we optimize numerically.

\section{D.10 The Special Case $\chi=0$}

We now provide a general formulation the allocation and planning problem without hand-to-mouth agents as an important special case.

We start by recording several important parameters. For $\chi=0$, we have $\Lambda_{y}=\frac{1}{1-v}, \Lambda_{\tau}=0$, $\Lambda_{s}=1, \Lambda_{g}=\frac{v}{1-v}$ and $\Lambda_{\theta}=1-\alpha$. Furthermore, we have $v_{\tau}=0, v_{y}=\frac{1}{1-v}, v_{\theta}=-(1-\alpha)$, $v_{g}=-\frac{v}{1-v}$. And $\zeta_{y}=\frac{1-\alpha}{1-v}, \zeta_{g}=-(1-\alpha) \frac{v}{1-v}, \zeta_{\tau}=0$, and $\zeta_{\theta}=1-(1-\alpha)^{2}$.

\section{D.10.1 General Allocation for $\chi=0$}

Give these parameters, we can write the (IS) equation for $\chi=0$ as

$$
\frac{1}{1-v} \dot{\hat{\bar{y}}}_{t}^{i}=-\left(\hat{\bar{\pi}}_{H, t}^{i}+\dot{\tilde{s}}_{t}^{i}\right)+\frac{v}{1-v} \dot{\hat{\bar{g}}}_{t}^{i}+(1-\alpha) \dot{\hat{\hat{\theta}}}_{t}^{i}
$$

For the Phillips Curve, we record the following parameters: $\kappa_{y}=\lambda\left(\phi+\frac{1}{1-v}\right), \kappa_{g}=-\lambda \frac{v}{1-v}$, $\kappa_{\tau}=0$, and $\kappa_{\theta}=\alpha \lambda$. Therefore, we can write the (NKPC) as

$$
\dot{\hat{\bar{\pi}}}_{H, t}^{i}=\rho \hat{\bar{\pi}}_{H, t}^{i}-\lambda\left(\phi+\frac{1}{1-v}\right) \hat{y}_{t}^{i}-\alpha \lambda \hat{\theta}_{t}^{i}+\lambda \frac{v}{1-v} \hat{g}_{t}^{i}
$$

The initial condition is the given by

$$
\frac{1}{1-v} \hat{\bar{y}}_{0}^{i}=-\tilde{s}_{0}^{i}+\frac{v}{1-v} \hat{\bar{g}}_{0}^{i}+(1-\alpha) \hat{\bar{\theta}}_{0}^{i} .
$$


Finally, for country i's budget constraint, we note that for $\chi=0, \Gamma_{y}=-\frac{\alpha v}{1-v}, \Gamma_{\tau}=1, \Gamma_{\theta}=$ $1-\alpha-(1-v)-v(1-\alpha)^{2}$, and $\Gamma_{g}=v+\alpha \frac{v^{2}}{1-v}$. We can then use the government budget constraint to substitute in for $\hat{\bar{\tau}}_{t}^{i, 0}$, which yields that in the absence of transfers we have the following NFA condition

$$
0=-\alpha(1-v) \int_{0}^{\infty} e^{-\rho t} \hat{\theta}_{t}^{i} d t
$$

\section{D.10.2 Loss Function and Planning Problem for $\chi=0$}

For $\chi=0$, the loss function coefficients are as follows: $\alpha_{\tau \tau}=\alpha_{g \tau}=\alpha_{y \tau}=\alpha_{\tau \theta}=\alpha_{y \theta}=\alpha_{g \theta}=0$. This leaves us with

$$
\begin{aligned}
\alpha_{\pi} & =\frac{\epsilon}{2 \lambda} \\
\alpha_{y y} & =-\frac{1+(1-v) \phi}{2(1-v)} \\
\alpha_{g g} & =-\frac{v}{2(1-v)} \\
\alpha_{\theta \theta} & =-\frac{1-v}{2}\left(1-(1-\alpha)^{2}\right) \\
\alpha_{y g} & =\frac{v}{1-v} .
\end{aligned}
$$

Hence, the loss function can be written as

$$
\begin{gathered}
\mathbb{L}=\int_{0}^{\infty} \int_{0}^{1} e^{-\rho t}\left[\frac{\epsilon}{2 \lambda}\left(\hat{\bar{\pi}}_{H, t}^{i}\right)^{2}+\frac{1+(1-v) \phi}{2(1-v)}\left(\hat{\bar{y}}_{t}^{i}\right)^{2}+\frac{1-v}{2}\left(1-(1-\alpha)^{2}\right)\left(\hat{\bar{\theta}}_{t}^{i}\right)^{2}\right. \\
\left.+\frac{v}{2(1-v)}\left(\hat{\bar{g}}_{t}^{i}\right)^{2}-\frac{v}{1-v} \hat{\bar{y}}_{t}^{i} \hat{\bar{g}}_{t}^{i}\right] d i d t .
\end{gathered}
$$

where of course $a_{t}^{i}=e^{-\psi t} a_{0}^{i}$ is the initial productivity shock.

\section{D.10.3 Planning Problem for Transfers when $\chi=0$}

When the social planner can only use transfers, we have $\hat{\bar{g}}_{t}^{i}=0$ and $\hat{\bar{\theta}}_{t}^{i}=\hat{\bar{\theta}}^{i}$. Therefore, we can write the planning problem as

$$
\min _{\hat{\theta}^{i}} \int_{0}^{\infty} \int_{0}^{1} e^{-\rho t}\left[\frac{\epsilon}{2 \lambda}\left(\hat{\bar{\pi}}_{H, t}^{i}\right)^{2}+\frac{1+(1-v) \phi}{2(1-v)}\left(\hat{\bar{y}}_{t}^{i}\right)^{2}+\frac{1-v}{2}\left(1-(1-\alpha)^{2}\right)\left(\hat{\bar{\theta}}^{i}\right)^{2}\right] d i d t,
$$

subject to

$$
\begin{aligned}
\frac{1}{1-v} \dot{\hat{\bar{y}}}_{t}^{i} & =-\left(\hat{\bar{\pi}}_{H, t}^{i}+\dot{\tilde{s}}_{t}^{i}\right) \\
\dot{\bar{\pi}}_{H, t}^{i} & =\rho \hat{\bar{\pi}}_{H, t}^{i}-\lambda\left(\phi+\frac{1}{1-v}\right) \hat{\bar{y}}_{t}^{i}-\alpha \lambda \hat{\bar{\theta}}^{i}
\end{aligned}
$$




$$
\frac{1}{1-v} \hat{\bar{y}}_{0}^{i}=-\tilde{s}_{0}^{i}+(1-\alpha) \hat{\bar{\theta}}^{i}
$$

\section{D.10.4 Planning Problem for Capital Controls when $\chi=0$}

When the social planner can only use transfers, we have $\hat{\bar{g}}_{t}^{i}=0$ and $\hat{\bar{\tau}}_{t}^{i, r}=v \alpha \hat{\bar{s}}_{t}^{i}$. Therefore, we can write the planning problem as

$$
\min _{\left\{\hat{\theta}_{t}^{i}\right\}_{t \geq 0}} \int_{0}^{\infty} \int_{0}^{1} e^{-\rho t}\left[\frac{\epsilon}{2 \lambda}\left(\hat{\bar{\pi}}_{H, t}^{i}\right)^{2}+\frac{1+(1-v) \phi}{2(1-v)}\left(\hat{\bar{y}}_{t}^{i}\right)^{2}+\frac{1-v}{2}\left(1-(1-\alpha)^{2}\right)\left(\hat{\bar{\theta}}_{t}^{i}\right)^{2}\right] d i d t,
$$

subject to

$$
\begin{aligned}
\frac{1}{1-v} \dot{\hat{y}}_{t}^{i} & =-\left(\hat{\bar{\pi}}_{H, t}^{i}+\dot{\tilde{s}}_{t}^{i}\right)+(1-\alpha) \dot{\hat{\theta}}_{t}^{i} \\
\dot{\hat{\bar{\pi}}}_{H, t}^{i} & =\rho \hat{\bar{\pi}}_{H, t}^{i}-\lambda\left(\phi+\frac{1}{1-v}\right) \hat{\bar{y}}_{t}^{i}-\alpha \lambda \hat{\bar{\theta}}_{t}^{i} \\
\frac{1}{1-v} \hat{\bar{y}}_{0}^{i} & =-\tilde{s}_{0}^{i}+(1-\alpha) \hat{\bar{\theta}}_{0}^{i} \\
0 & =-\alpha(1-v) \int_{0}^{\infty} e^{-\rho t} \hat{\bar{\theta}}_{t}^{i} d t .
\end{aligned}
$$

\section{D.10.5 Planning Problem for Government Spending when $\chi=0$}

When the social planner can only use government spending, we have $\hat{\bar{\theta}}_{t}^{i}=0$ and $\hat{\bar{\tau}}_{t}^{i, r}=v \alpha \hat{\mathrm{s}}_{t}^{i}$. Therefore, we can write the planning problem as

$$
\min _{\left\{\hat{\theta}_{t}^{i}\right\}_{t \geq 0}} \int_{0}^{\infty} \int_{0}^{1} e^{-\rho t}\left[\frac{\epsilon}{2 \lambda}\left(\hat{\bar{\pi}}_{H, t}^{i}\right)^{2}+\frac{1+(1-v) \phi}{2(1-v)}\left(\hat{\bar{y}}_{t}^{i}\right)^{2}+\frac{v}{2(1-v)}\left(\hat{\bar{g}}_{t}^{i}\right)^{2}-\frac{v}{1-v} \hat{\bar{y}}_{t}^{i} \hat{\bar{g}}_{t}^{i}\right] d i d t,
$$

subject to

$$
\begin{aligned}
\frac{1}{1-v} \dot{\overline{\hat{y}}}_{t}^{i} & =-\left(\hat{\bar{\pi}}_{H, t}^{i}+\dot{\tilde{\mathrm{s}}}_{t}^{i}\right)+\frac{v}{1-v} \dot{\overline{\hat{g}}}_{t}^{i} \\
\dot{\bar{\pi}}_{H, t}^{i} & =\rho \hat{\bar{\pi}}_{H, t}^{i}-\lambda\left(\phi+\frac{1}{1-v}\right) \hat{\bar{y}}_{t}^{i}+\lambda \frac{v}{1-v} \hat{\bar{g}}_{t}^{i} \\
\frac{1}{1-v} \hat{\bar{y}}_{0}^{i} & =-\tilde{s}_{0}^{i}+\frac{v}{1-v} \hat{\bar{g}}_{0}^{i} .
\end{aligned}
$$

